ISBN 82-553-0842-3

Pure Mathematics
No.15

June 1993

\title{
SYMMETRIES
}

AND

INTEGRALS

\author{
by
}

B. Komrakov and V. Lychagin*

PREPRINT SERIES - Matematisk institutt, Universitetet i Oslo

* Submitted under the 1993 cooperation project between the Department of Mathematics, University of Oslo, and the International Sophus Lie Center. 
This publication was typeset using $\mathcal{A} \mathcal{M} \mathcal{S}$-TEX, the American Mathematical Society's TEX macro system. 

B. Komrakov V. LyChAGIN

\section{SYMMETRIES \\ AND \\ INTEGRALS}

International Sophus Lie Center 


\title{
SYMMETRIES AND INTEGRALS
}

\author{
B. KOMRAKOV \\ V. LYCHAGIN
}

Translated to English by L. Levin

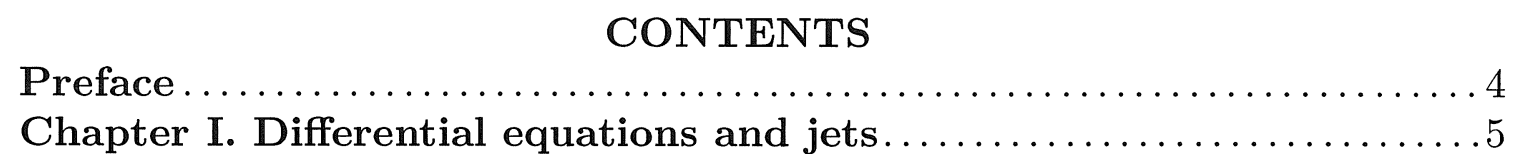

1.1. Geometrical interpretation of ordinary differential equations $\ldots \ldots \ldots \ldots \ldots \ldots$

1.2. Geometrical interpretation of systems of differential equations . . . . . . . . . 16

1.3. Completely integrable systems of differential equations and distributions $\ldots 20$

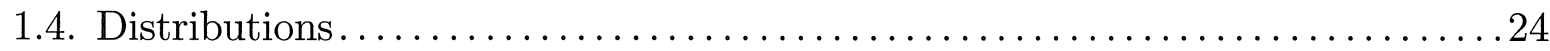

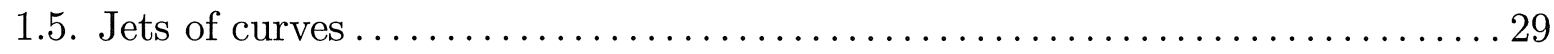

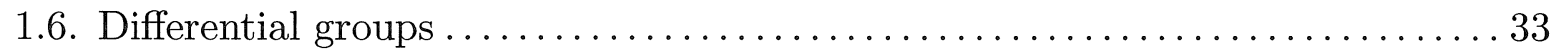

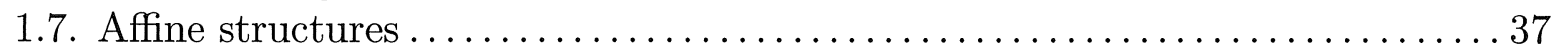

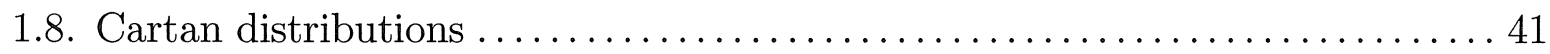

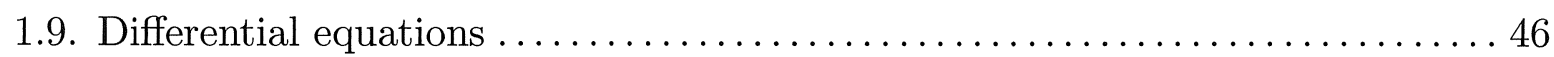

Chapter II. Symmetries ................................ 50

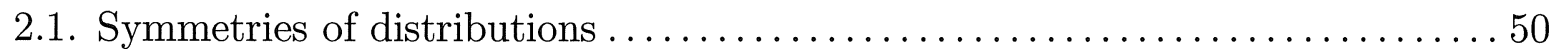

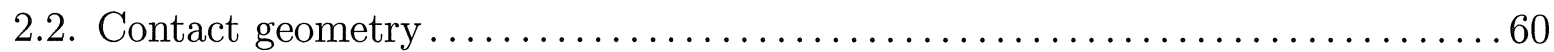

2.3. Lie fields and symmetries of Cartan distributions . . . . . . . . . . . . . . 68

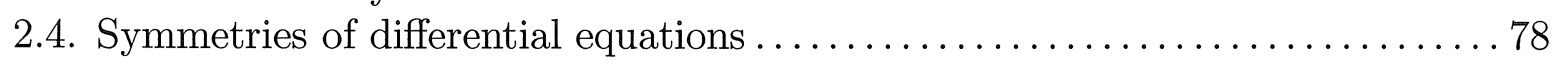

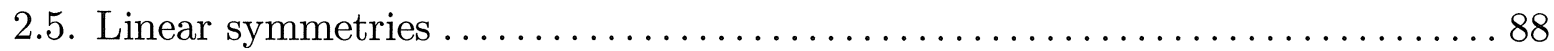

Chapter III. Integrals . . . . . . . . . . . . . . . . . . . . . . . . 96

3.1. Integrals of completely integrable distributions $\ldots \ldots \ldots \ldots \ldots \ldots \ldots \ldots \ldots \ldots \ldots$

3.2. Lie-Bianchi theorem ........................................... 105

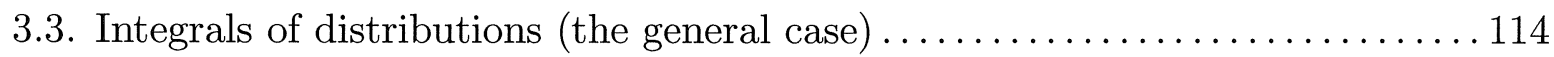

3.4. Differential invariants ..................................... 117

3.5. Lagrange integrals and supersymmetries...................... 127

Chapter IV. Models ...................................... 137

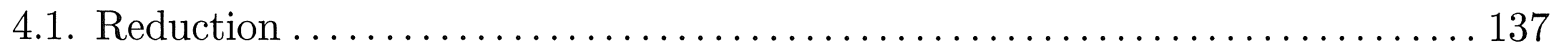

4.2. Model equations and the superposition principle................... 146

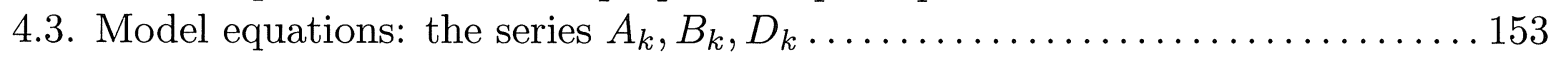

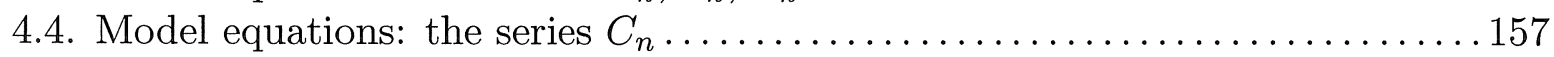




\section{Preface}

This text constitutes a preliminary version of an introductory textbook of the solution of ordinary differential equations using symmetries, and is primerely aimed for third year students of the ISLC college.

This book is not self-supported (one must know some analysis on manifolds, including differential forms) and does not contain references.

First of all we recommend the study of Sophus Lie's original works (which unfortunately are not easy reading), since the the further development of this field of mathematics often consisted of the rediscovery of his results, but the main part of his results were not exploited.

We suggest some books which might be useful in order to gain individual understanding of this subject:

1. P. Olver, Applications of Lie groups to differential equations, (Graduate Texts in Mathematics 107), Springer, New York, 1986.

2. V. Lychagin, Lectures on geometry of differential equations. Part I, Roma, 1992.

3. N. Ovsiannikov, Group analysis of differential equations, Academic, New York, 1982.

4. H. Stephani, Differential equations. Their solution using symmetries, Cambridge University Press, Springer, New York, 1990.

5. N. Ibragimov, Transformation groups applied to mathematical physics, Reidel, Boston, 1985.

6. B. Komrakov, Primitive actions and Sophus Lie problem, Preprint series, Inst. of Mathematics. Univ. of Oslo, 1993.

7. B. Komrakov, A. Churyumov, B. Doubrov, Two-dimensional homogeneous spaces, Preprint series, Inst. of Mathematics. Univ. of Oslo, 1993. 
CHAPTER I

\section{DIFFERENTIAL EQUATIONS AND JETS}

\section{§1. Geometrical interpretation of ordinary Differential EQUATIONS}

1.1. Let us consider differential equations of the first order with respect to a realvalued function defined on $\mathbb{R}$. They form the simplest class of ordinary differential equations. An equation of this kind solvable with respect to a derivative can be written as

$$
y^{\prime}=F(x, y)
$$

where $x \in \mathbb{R}$ and $y=y(x)$ is a function to be found.

It is well known that equation (1) can be geometrically interpreted as a directional field. In this (standard) interpretation a triple $\left(x, y(x), y^{\prime}(x)\right)$ is interpreted as the point $(x, y)$ on the plane together with the straight line passing through this point and tangent to the graph of the function $y(x)$ (see Fig. 1).

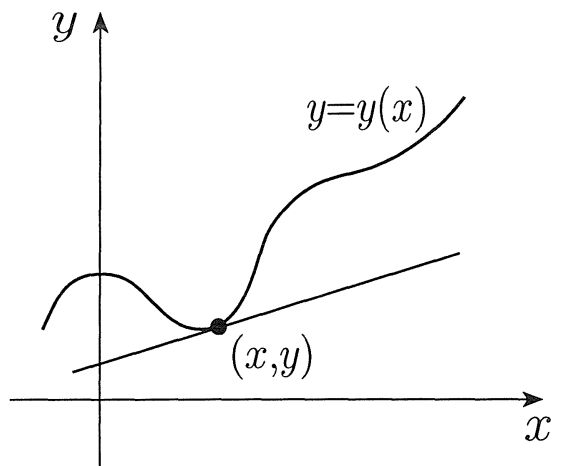

Fig. 1

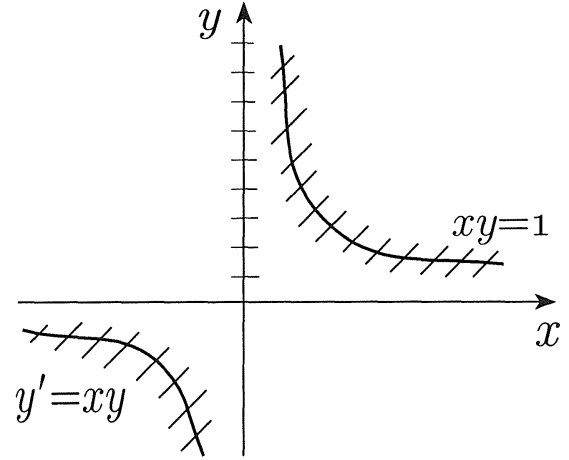

Fig. 2

Thus, at each point $(x, y)$ of the plane, differential equation (1) determines a straight line with a slope equal to $F(x, y)$ (see Fig. 2). Under this approach, the procedure of solution consists in finding a function $y=h(x)$ such that tangent lines to its graph at points $(x, h(x))$ coincide with the corresponding straight lines of the given directional field.

Suppose that a differential equation cannot be solved with respect to a derivative $y^{\prime}$, i.e. it has the form

$$
H\left(x, y(x), y^{\prime}(x)\right)=0
$$

and the function $\partial H / \partial y^{\prime}$, say, vanishes. Then the standard geometrical interpretation turns out to be rather awkward, since now at each point of the plane there may be several (or even infinitely many) straight lines, their slopes $k$ at a fixed point $(x, y)$ 
being determined from the relation

$$
H(x, y, k)=0
$$

1.2. Example. Consider the differential equation

$$
\left(y^{\prime}\right)^{2}+2 y^{\prime}+x y=0
$$

In this case, the directional field is defined only on that part of the plane where $x y \leqslant 1$. In addition, on the interior of the domain $(x y<1)$ there exist exactly two different directions:

$$
k_{ \pm}=-1 \pm \sqrt{1-x y}
$$

whereas on the hyperbola $x y=1$ only one: $k=-1$. (See Fig. 3.)

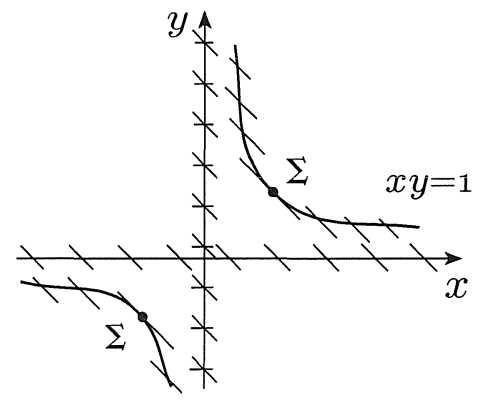

Fig. 3

In order to simplify the procedure of solution of similar differential equations and make it clearer from geometrical point of view, we introduce the space $J^{1}$, which coincides with the three-dimensional arithmetic space, its elements $(x, y, p)$ being identified with pairs:

$$
[(x, y) ; l(x, y, p)]
$$

where $l(x, y, p)$ is the straight line passing through the point $(x, y)$, its slope being equal to $p$ (see Fig. 4 ).

We shall say that $J^{1}$ is the space of 1 -jets of functions, and its elements (4) will be called 1-jets (of functions).

Differential equation (2) can now be regarded as the surface $\mathcal{E}$ in $J^{1}$ defined by

$$
H(x, y, p)=0
$$

and its solutions $y=h(x)$ as curves of the form

$$
L_{h}=\left\{y=h(x), p=h^{\prime}(x)\right\}
$$

lying on $\mathcal{E}$. 


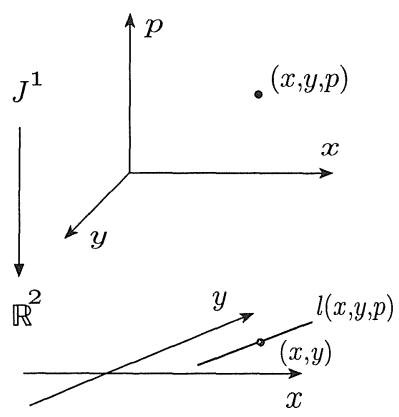

Fig. 4

In the example considered before the surface $\mathcal{E}$ is the one-sheet hyperboloid and solutions of equation (3) are curves of the form $L_{h}$ lying on the hyperboloid.

1.3. We are now going to construct the analogue of a directional field. Preparatory to this we shall give an internal (in terms of the space of 1 -jets) characteristic of those curves in $J^{1}$ that have form (6) for a certain smooth function $h(x)$. Fix a point

$$
a=\left(x_{0}, y_{0}, p_{0}\right) \in J^{1}
$$

and consider tangent vectors at the point $a$ to various curves of the form $L_{n}$ passing through $a$. It is easy to see that these vectors have the form

$$
v=\left(1, p_{0}, h^{\prime \prime}\left(x_{0}\right)\right)
$$

Under the condition that $h^{\prime \prime}\left(x_{0}\right)$ be arbitrary, all linear combinations of vectors $(7)$ form the 2-dimensional plane $C(a)$ given by the equation

$$
y-y_{0}=p_{0}\left(x-x_{0}\right) .
$$

The plane $C(a)$ is called the Cartan plane at the point $a$. The field of Cartan planes

$$
C: a \mapsto C(a)
$$

on the space $J^{1}$ of 1 -jets is called the Cartan distribution. Relation (8) shows that the Cartan distribution can be given by the following differential form of degree one:

$$
\omega=d y-p d x
$$

in the sense that

$$
C(a)=\operatorname{ker} \omega_{a}=\left\{v \in \mathrm{T}_{a} J^{1} \mid \omega_{a}(v)=0\right\} .
$$

The differential form $\omega$ is called the Cartan form.

It is immediate from construction of the Cartan distribution that each curve of the form $L_{h}$ in $J^{1}$ is an integral curve of the distribution; in other words, for any point $a \in L_{h}$ the tangent vector to $L_{n}$ at $a$ belongs to the Cartan plane $C(a)$. The converse is also true. 
Proposition. Let $L$ be a curve in $J^{1}$ such that

(i) $L$ is an integral curve of the Cartan distribution;

(ii) $L$ can be "well" projected on the $x$-axis, i.e. the $x$-coordinate can be chosen as a parameter on $L$.

Then there exists a smooth function $h=h(x)$ such that $L=L_{h}$.

Proof. Condition (ii) means that the curve $L$ can be given by equations

$$
y=f(x), \quad p=g(x) .
$$

Condition (i) implies that the Cartan form $\omega$ vanishes on $L$ :

$$
\left.\omega\right|_{L}=d f-g d x=\left(f^{\prime}(x)-g(x)\right) d x=0 .
$$

Therefore, $g=f^{\prime}$ and

$$
L=L_{f}=\left\{y=f(x), p=f^{\prime}(x)\right\}
$$

1.4. Now we have everything necessary for construction of a directional field.

Each solution $y=h(x)$ of equation (2) gives the curve $L_{h}$, which lies on the surface $\mathcal{E}$. In addition, at each point $b \in L_{h}$ the curve $L_{h}$ is tangent to the corresponding Cartan plane $C(b)$. Now, for each point $a$ of the surface $\mathcal{E}$ consider the intersection of the tangent plane $\mathrm{T}_{a} \mathcal{E}$ and Cartan plane $C(a)$. We see that the tangent vector to $L_{h}$ at $a$ belongs to the intersection. In general, the planes $\mathrm{T}_{a} \mathcal{E}$ and $C(a)$ do not coincide and, therefore, their intersection

$$
l(a)=\mathrm{T}_{a} \mathcal{E} \cap C(a)
$$

is a straight line (see Fig. 5).

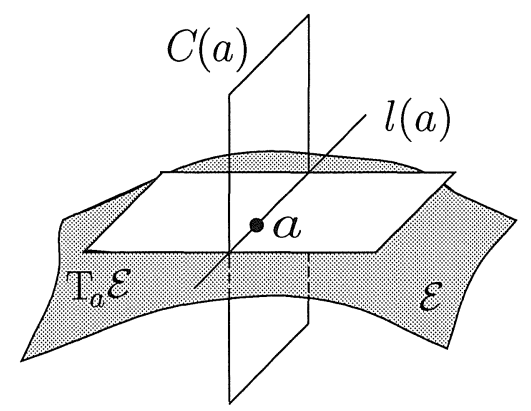

Fig. 5

More exactly, we shall say that a point $a \in \mathcal{E}$ is regular if

$$
\mathrm{T}_{a} \mathcal{E} \neq C(a)
$$

otherwise, we shall say that $a$ is singular. 
Relation (10) determines the directional field $l: a \mapsto l(a)$ at regular points of the equation $\mathcal{E}$ (see Fig. 6).

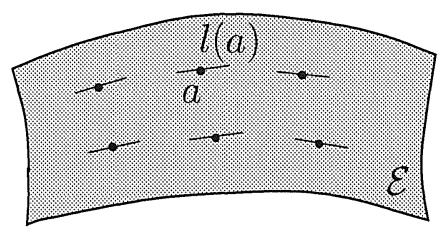

Fig. 6

A solution of our differential equation is a curve on $\mathcal{E}$ with the following properties: tangent lines to it coincide with the corresponding straight lines of the directional field $l$ (condition $(i)$ of the proposition above) and it can be "well" projected on the $x$-axis (condition $(i i)$ ).

Note that the last condition is totally dependent on $l$ and does not necessarily hold.

1.5. Let $a \in \mathcal{E}$ and let $L$ be a solution passing through $a$. We shall say that $L$ is regular if it satisfies condition $(i i)$. Let us find conditions for $a$ and $L$ to be regular in terms of the function $H(x, y, p)$, which gives our differential equation. A point $a \in \mathcal{E}$ is regular if and only if the following differential 1 -forms are linearly independent at a:

$$
\left\{\begin{array}{l}
d H=H_{x} d x+H_{y} d y+H_{p} d p, \\
\omega=-p d x+d y .
\end{array}\right.
$$

Therefore, $a \in \mathcal{E}$ is singular if and only if

$$
H_{p}=0 \quad \text { and } \quad H_{x}+p H_{y}=0
$$

at the point $a$.

Further, the fact that a solution $L$ passing through a regular point $a \in \mathcal{E}$ is regular at $a$ means that the projection of the straight line $l(a)$ on the $x$-axis is other than zero.

Assume the contrary. Then $l(a)$ is spanned by a vector of the form

$$
v=\alpha \frac{\partial}{\partial y}+\beta \frac{\partial}{\partial p}
$$

Since $l(a)$ lies on the Cartan plane $C(a)$, we have

$$
\omega_{a}(v)=\alpha=0
$$

Therefore, it can be assumed that $\alpha=0$ and $\beta=1$. Since $v$ is a tangent vector to $\mathcal{E}$, we have

$$
d H(v)=0
$$


and therefore

$$
H_{p}=0
$$
if

Thus, a curve $L$ passing through a regular point $a$ does not give a regular solution

$$
H_{x}+p H_{y} \neq 0 \text { and } H_{p}=0
$$

at the point $a$. Now let $a$ be a regular point of $\mathcal{E}$. After solving the system of linear equations $\omega_{a}=0, d_{a} H=0$, we see that the following vector can always be chosen as a generator of the straight line $l_{a}$ :

$$
Y_{H}=-H_{p} \frac{\partial}{\partial x}-p H_{p} \frac{\partial}{\partial y}+\left(H_{x}+p H_{y}\right) \frac{\partial}{\partial p}
$$

It is easy to see that the vector field $Y_{H}$ is tangent to $\mathcal{E}$. It is called a characteristic vector field.

1.6. Summarizing all the observations above, we can give a geometrical picture that accompanies the procedure of solution of differential equation (2). Let $\mathcal{E} \subset J^{1}$ be the surface corresponding to the given differential equation and let $l$ denote directional field (15), which is defined at regular points of $\mathcal{E}$. Then solutions of differential equation (2) are integral curves of this directional field. Moreover, these solutions are smooth singlevalued functions at all points except those where condition (14) holds (see Fig. 7).

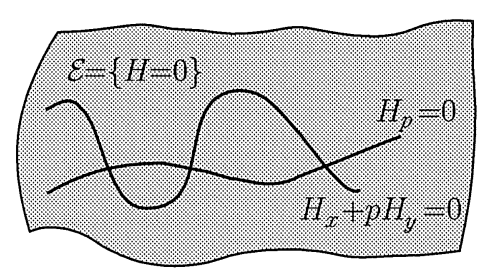

Fig. 7

Let us distract ourselves from singular points of equations for a while and by solutions of the differential equation $\mathcal{E}$ mean integral curves $L$ of the directional field $l$ that do not necessarily satisfy condition (ii) of proposition 1.3. This way we arrive at the natural geometrical generalization of the concept of a solution of a differential equation - a many-valued solution. Indeed, let $L$ be an integral curve that does not satisfy condition $(i i)$. The projection of $L$ on the plane $(x, y)$ is a curve with singularities at exactly those points where condition $(i i)$ does not hold; this projection can be regarded as a graph of a many-valued function (see Fig. 8). 


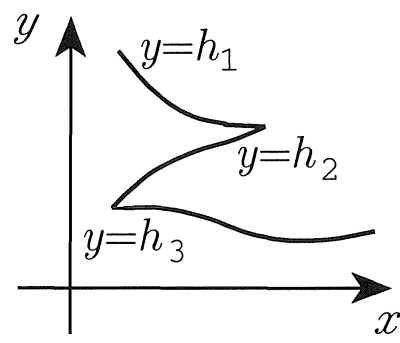

Fig. 8

1.7. Example. First, consider the differential equation

$$
\left(y^{\prime}\right)^{2}-x=0
$$

which can be easily integrated. The surface $\mathcal{E} \subset J^{1}$ corresponding to the equation is the parabolic cylinder

$$
p^{2}-x=0
$$

The functions $y$ and $p$ can be chosen as coordinates on $\mathcal{E}$, and $\mathcal{E}$ can be identified with the plane $\mathbb{R}^{2}(y, p)$ by means of the mapping

$$
\begin{aligned}
\mathcal{E}=\left\{\left(p^{2}, y, p\right)\right\} & \stackrel{\sim}{\rightarrow} \mathbb{R}^{2}(y, p) \\
\left(p^{2}, y, p\right) & \mapsto(y, p)
\end{aligned}
$$

The corresponding characteristic vector field has the form

$$
Y_{H}=-2 p \frac{\partial}{\partial x}-2 p^{2} \frac{\partial}{\partial y}-\frac{\partial}{\partial p}
$$

Therefore, the projection of the directional field $l$ from $\mathcal{E}$ on the plane $\mathbb{R}^{2}(y, p)$ determines the directional field on $\mathbb{R}^{2}(y, p)$ :

$$
\tilde{l}:(y, p) \mapsto \tilde{l}(y, p),
$$

where the straight line $\tilde{l}(y, p)$ is spanned by the vector

$$
\tilde{Y}_{H}=-2 p^{2} \frac{\partial}{\partial y}-\frac{\partial}{\partial p}
$$

Identifying integral curves of $\tilde{l}$ with graphs of functions $y=g(p)$, we obtain the following differential equation with respect to $g(p)$ :

$$
g^{\prime}=2 p^{2}
$$


It follows that

$$
g(p)=\frac{2}{3} p^{3}+c
$$

Solutions, regarded as functions of $x$, have singularities at those points where

$$
H_{p}=2 p=0
$$

Finally we obtain

$$
\left(y^{\prime}\right)^{2}=x \Rightarrow y^{\prime}= \pm \sqrt{x} \Rightarrow y= \pm \frac{2}{3} x^{3 / 2}+q_{1}
$$

Thus, solutions of our equation are semicubical parabolas, their cuspidal points lying on the $y$-axis (see Fig. 9).

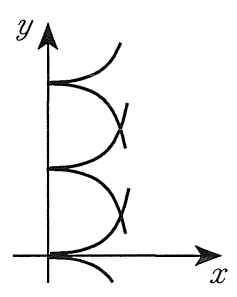

Fig. 9

1.8. Example. Let the function $H$ of differential equation (2) has the form

$$
H=p^{2}+2 p+x y
$$

The set $\Sigma$ of singular points of the equation can be found from the following relations:

$$
\left\{\begin{array}{l}
H=p^{2}+2 p+x y=0 \\
H_{p}=2 p+2=0 \\
H_{x}+p H_{y}=y+x p=0 .
\end{array}\right.
$$

It follows that

$$
x=y, \quad p=-1, \quad y= \pm 1 .
$$

The projection of the set $\Sigma$ on the plane $(x, y)$ consists of two points $x=y=1$, $x=y=-1$, which are exactly the points of tangency of the hyperbola $x y=1$ and the directional field (see Fig 3).

Since $H_{p}=2 p+2$, we have exactly one singular curve:

$$
\{p=-1, x y=1\} .
$$


Its projection on the plane $(x, y)$ is the hyperbola $x y=1$. Because of this, solutions have singularities at the points of this hyperbola and become many-valued there.

1.9. Basic constructions considered earlier can be automatically extended to the case of an ordinary differential equation of an arbitrary order $k(k \geqslant 2)$.

Each ordinary differential equation of the $k$-th order can be written as

$$
H\left(x, y, y^{\prime}, \ldots, y^{(k)}\right)=0 .
$$

For $x^{0} \in \mathbb{R}$ and a smooth function $g$, consider the $(k+2)$-tuple

$$
[g]_{x^{0}}^{k}=\left(x^{0}, g\left(x^{0}\right), g^{\prime}\left(x^{0}\right), \ldots, g^{(k)}\left(x^{0}\right)\right) \text {. }
$$

We shall call it the $k$-jet of the function $g$ at the point $x^{0}$. By $J^{k}$ denote the set of all $k$-jets at all possible points. We shall call it the space of $k$-jets. It can be considered as the arithmetic $(k+2)$-space $\mathbb{R}^{k+2}$ with the natural coordinates $\left(x, p_{0}, p_{1}, \ldots, p_{k}\right)$ where

$$
p_{j}\left([g]_{x^{0}}^{k}\right)=g^{(j)}\left(x^{0}\right) .
$$

Thus, the function $H$ is a function defined on the space $J^{k}$ and equation (16) gives the hypersurface $\mathcal{E}$ in $J^{k}$ defined by

$$
\mathcal{E}=\left\{\left(x, p_{0}, p_{1}, \ldots, p_{k}\right) \mid H\left(x, p_{0}, p_{1}, \ldots, p_{k}\right)=0\right\}
$$

Solutions $y=h(x)$ of differential equation (16) can be considered as curves on $\mathcal{E} \subset J^{k}$ of the form

$$
L_{h}^{(k)}=\left\{p_{0}=h(x), p_{1}=h^{\prime}(x), \ldots, p_{k}=h^{(k)}(x)\right\} .
$$

In order to distinguish curves of this form from other smooth curves in $J^{k}$, fix a point

$$
a=\left(x^{0}, p_{0}^{0}, p_{1}^{0}, \ldots, p_{k}^{0}\right) \in J^{k}
$$

and consider tangent vectors at $a$ to various curves of the form $L_{h}^{(k)}$ passing through a. Since these tangent vectors have the form

$$
v=\left(1, p_{1}^{0}, p_{2}^{0}, \ldots, p_{k}^{0}, h^{(k+1)}\left(x^{0}\right)\right)
$$

we see that their linear combinations form the 2-dimensional plane $C(a)$ given by the following equations:

$$
\left\{\begin{array}{l}
p_{0}-p_{0}^{0}=p_{1}^{0}\left(x-x^{0}\right), \\
p_{1}-p_{1}^{0}=p_{2}^{0}\left(x-x^{0}\right), \\
\vdots \\
p_{k-1}-p_{k-1}^{0}=p_{k}^{0}\left(x-x^{0}\right) .
\end{array}\right.
$$


The plane $C(a)$ is called the Cartan plane at the point $a$, and the distribution $C: a \mapsto$ $C(a)$ is called the Cartan distribution in the space $J^{k}$ of $k$-jets. From equations (21) it follows that the Cartan distribution $C$ can also be defined as the set of common zeroes of the following set of differential 1-forms on $J^{k}$ :

$$
\left\{\begin{array}{l}
\omega_{0}=d p_{0}-p_{1} d x \\
\omega_{1}=d p_{1}-p_{2} d x \\
\vdots \\
\omega_{k-1}=d p_{k-1}-p_{k} d x .
\end{array}\right.
$$

The differential forms $\omega_{j}, j=0, \ldots, k-1$, are called the Cartan forms.

1.10. Proposition. Let $L$ be a curve in $J^{k}$. The curve $L$ has the form $L_{h}^{(k)}$ for some smooth function $h(x)$ if and only if the following two conditions are satisfied:

(i) $L$ is an integral curve of the Cartan distribution; in other words, for each point $a \in L$ the tangent vector to $L$ at a belongs to the Cartan plane $C(a)$;

(ii) the curve $L$ can be "well" projected on the $x$-axis.

Proof. The necessity of these conditions is clear. Let us prove their sufficiency. Condition ( $i i)$ implies that the function $x$ can be chosen as a parameter on the curve $L$. Therefore, $L$ can be given by the following system of equations:

$$
p_{0}=f_{0}(x), p_{1}=f_{1}(x), \ldots, p_{k}=f_{k}(x) .
$$

By virtue of condition $(i)$ we have

$$
\left.\omega_{j}\right|_{L}=d f_{j}-f_{j+1} d x=\left(f_{j}^{\prime}-f_{j+1}\right) d x=0
$$

for all $j \in\{0, \ldots, k-1\}$. Thus, $f_{j+1}=f_{j}^{\prime}$ and therefore $L=L_{h}^{(k)}$, where $h=f_{0}$.

1.11. So, necessary and sufficient conditions for a curve $L$ in $J^{k}$ to determine a solution of differential equation (16) are

(1) $L$ is an integral curve of the Cartan distribution;

(2) $L$ lies on the hypersurface $\mathcal{E} \subset J^{k}$;

(3) $L$ can be "well" projected on the $x$-axis.

Conditions (1) and (2) can be unified if we consider the restriction of the Cartan distribution to the equation $\mathcal{E}$. For each point $a \in \mathcal{E}$ consider the intersection of the hypersurface $\mathrm{T}_{a} \mathcal{E}$ tangent to $\mathcal{E}$ at $a$ and the Cartan plane $C(a)$.

We shall say that a point $a \in \mathcal{E}$ is regular if $C(a) \not \subset \mathrm{T}_{a} \mathcal{E}$; otherwise, it will be called a singular point.

Then for every regular point $a \in \mathcal{E}$ the intersection $\mathrm{T}_{a} \mathcal{E} \cap C(a)$ is a straight line $l(a)$, and the correspondence

$$
l: a \in \mathcal{E}_{r e g} \mapsto l(a)=\mathrm{T}_{a} \mathcal{E} \cap C(a)
$$


gives a directional field on the set of all regular points $\mathcal{E}_{r e g} \subset \mathcal{E}$.

Thus, from the geometrical standpoint, solutions of the differential equation (16) are those integral curves of the directional field $l$ that can be "well" projected on the $x$ axis. As earlier, omitting the last condition, we arrive at a geometrical generalization of the concept of a solution - a many-valued solution.

1.12. Let us find a condition for a point $a \in \mathcal{E}$ to be regular. The intersection of the Cartan plane $C(a)$ and the tangent hypersurface $\mathrm{T}_{a} \mathcal{E}$ can be found as the general solution of the system of linear equations

$$
\left\{\begin{array}{l}
\omega_{0}=d p_{0}-p_{1} d x=0, \\
\omega_{1}=d p_{1}-p_{2} d x=0 \\
\vdots \\
\omega_{k-1}=d p_{k-1}-p_{k} d x=0, \\
d H=H_{x} d x+H_{p_{0}} d p_{0}+\cdots+H_{p_{k}} d p_{k}=0 .
\end{array}\right.
$$

The differential 1-form $d H$ will be a linear combination of the Cartan forms only at singular points of the equation. Therefore, singular points can be found from the following equations:

$$
\left\{\begin{array}{l}
H_{x}+p_{1} H_{p_{0}}+\cdots+p_{k} H_{p_{k-1}}=0, \\
H_{p_{k}}=0 .
\end{array}\right.
$$

As a generator of the directional field $l$ (considered at regular points $a \in \mathcal{E}$ ) we can choose the vector field

$$
Y_{H}=-H_{p_{k}}\left(\frac{\partial}{\partial x}+p_{1} \frac{\partial}{\partial p_{0}}+\cdots+p_{k} \frac{\partial}{\partial p_{k-1}}\right)+\frac{d H}{d x} \frac{\partial}{\partial p_{k}}
$$

where by

$$
\frac{d H}{d x}=\frac{\partial H}{\partial x}+p_{1} \frac{\partial H}{\partial p_{0}}+\cdots+p_{k} \frac{\partial H}{\partial_{k-1}}
$$

we denote the "total" derivative with respect to $x$. The vector field $Y_{H}$ is called the characteristic vector field of equation (16). It can be easily verified that $Y_{H}$ is tangent to the hypersurface $H=0$.

Let $L$ be a solution passing through a point $a \in \mathcal{E}$. Let us consider a condition for $L$ to be regular at $a$, i.e. a condition for $L$ that it can be locally represented as $L_{h}^{(k)}$ for some smooth function $h(x)$. As in the case of first-order equations, this condition has the form

$$
H_{p_{k}} \neq 0
$$

Note that the characteristic vector field $Y_{H}$ (regarded as a derivation) can be written more briefly in the hamiltonian form:

$$
Y_{H}=-\frac{d H}{d p_{k}} \frac{d}{d x}+\frac{d H}{d x} \frac{\partial}{\partial p_{k}}
$$


where

$$
\frac{d}{d x}=\frac{\partial}{\partial x}+p_{1} \frac{\partial}{\partial p_{0}}+\cdots+p_{k} \frac{\partial}{\partial p_{k-1}}
$$

is the total derivative operator.

1.13. Example. Consider the Gauss hypergeometric equation

$$
x(1-x) y^{\prime \prime}+(c-(a+b+1) x) y^{\prime}-a b y=0,
$$

where $a, b, c \in \mathbb{R}$ are constants. The corresponding function $H$ has the form

$$
H=x(1-x) p_{2}+(c-(a+b+1) x) p_{1}-a b p_{0} .
$$

Thus, the equation $H=0$ gives a cubic surface in $J^{2}=\mathbb{R}^{4}$. The characteristic vector field of the equation has the form

$$
Y_{H}=-x(1-x) \frac{d}{d x}+\left[(c+1-(a+b+3) x) p_{2}-a b p_{1}\right] \frac{\partial}{\partial p_{2}} .
$$

Singular points can be found from the equations

$$
\left\{\begin{array}{l}
x(1-x)=0 \\
{[(c+1)-(a+b+3) x] p_{2}-(a+1)(b+1) p_{1}=0} \\
H=0
\end{array}\right.
$$

and form two straight lines:

$$
\begin{gathered}
l_{1}=\left\{x=0, a b p_{0}-c p_{1}=0,(a+1)(b+1) p_{1}-(c+1) p_{2}=0\right\} \\
l_{2}=\left\{x=1, a b p_{0}-(c-a-b-1) p_{1}=0,(a+1)(b+1) p_{1}-(c-a-b-2) p_{2}=0\right\} .
\end{gathered}
$$

Therefore, solutions of the hypergeometric equation lose their smoothness and become many-valued at points $\left(x, p_{0}, p_{1}, p_{2}\right)$ that belong to the compliment of the straight lines $l_{1}$ and $l_{2}$ in the planes

$$
\begin{gathered}
\Pi_{1}=\left\{x=0, c p_{1}-a b p_{0}=0\right\} \\
\Pi_{2}=\left\{x=1,(c-a-b-1) p_{1}-a b p_{0}=0\right\} .
\end{gathered}
$$

§2. Geometrical InTERPRETATION OF SYSTEMS OF DIFFERENTIAL EQUATIONS

In this section we shall apply the approach proposed in $\S 1$ to systems of differential equations and give their geometrical interpretation.

2.1. Fix a natural number $m \geqslant 1$. A system of ordinary differential equations of the $k$-th order with respect to a vector-valued function $Y: \mathbb{R} \rightarrow \mathbb{R}^{m}$ such that

$$
Y(x)=\left(\begin{array}{c}
y_{1}(x) \\
\vdots \\
y_{m}(x)
\end{array}\right)
$$


can be written as

$$
\left\{\begin{array}{l}
H_{1}\left(x, Y, Y^{\prime}, \ldots, Y^{(k)}\right)=0 \\
\vdots \\
H_{s}\left(x, Y, Y^{\prime}, \ldots, Y^{(k)}\right)=0 .
\end{array}\right.
$$

For each vector-valued function $Y(x)$ and point $x^{0} \in \mathbb{R}$ consider the vector

$$
[Y]_{x^{0}}^{k}=\left(x^{0}, Y\left(x^{0}\right), Y^{\prime}\left(x^{0}\right), \ldots, Y^{(k)}\left(x^{0}\right)\right)
$$

We shall call it the $k$-jet of the function $Y$ at the point $x^{0}$. The set of all $k$-jets of functions at all possible points is called the space of $k$-jets. We shall denote it by $J^{k}(m)$. If $m=1$ then $J^{k}(1)=J^{k}$. From (2) it follows that $J^{k}(m)$ is isomorphic to the arithmetic space $\mathbb{R}^{N}$, where $N=1+m(k+1)$. As coordinates in $J^{k}$ we choose the functions

$$
\left(x, p_{0}^{1}, \ldots, p_{0}^{m} ; p_{1}^{1}, \ldots, p_{1}^{m} ; \ldots ; p_{k}^{1}, \ldots, p_{k}^{m}\right),
$$

where the coordinate function $p_{j}^{i}, i=1, \ldots, m ; j=0, \ldots, k$, gives the $j$-th order derivative of the $i$-th component $y_{i}$ :

$$
p_{j}^{i}\left([Y]_{x^{0}}^{k}\right)=\left(y_{i}\right)^{(j)}\left(x^{0}\right) .
$$

The functions $H_{1}, \ldots, H_{s}$ involved in system (1) can be regarded as functions on $J^{k}(m)$. The system itself gives the submanifold $\mathcal{E}$ in $J^{k}(m)$ defined by the following relations:

$$
\left\{\begin{array}{l}
H_{1}\left(x, p_{0}^{1}, \ldots, p_{0}^{m} ; p_{1}^{1}, \ldots, p_{1}^{m} ; \ldots ; p_{k}^{1}, \ldots, p_{k}^{m}\right)=0 \\
\vdots \\
H_{s}\left(x, p_{0}^{1}, \ldots, p_{0}^{m} ; p_{1}^{1}, \ldots, p_{1}^{m} ; \ldots ; p_{k}^{1}, \ldots, p_{k}^{m}\right)=0 .
\end{array}\right.
$$

If the functions $H_{1}, \ldots, H_{s}$ are functionally independent, then the codimension of $\mathcal{E}$ in $J^{k}(m)$ equals $s$.

Solutions $Y=h(x)$ of system (1) are curves of the form

$$
\begin{aligned}
& L_{h}^{(k)}=\left\{p_{0}^{1}=h_{1}(x), \ldots, p_{0}^{m}=h_{m}(x), \ldots\right. \\
& \left.\qquad p_{k}^{1}=\left(h_{1}\right)^{(k)}(x), \ldots, p_{k}^{m}=\left(h_{m}\right)^{(k)}(x)\right\}
\end{aligned}
$$

lying in $\mathcal{E}$.

We shall say that a system of differential equations is determinate if codim $\mathcal{E}=s=$ $m$ and underdeterminate if $s<m$.

Let us note that although this book is devoted mainly to determinate systems of differential equations, this in no way means that underdeterminate ones are of little interest. On the contrary, these systems can be found in non-holonomic mechanics and in control theory and have very useful applications. However, their theory requires its own presentation and somewhat different approaches. 
2.2. Construction of the Cartan distribution

$$
C: a \in J^{k}(m) \mapsto C(a)
$$

in this case is very similar to that in the case $m=1$. It is the distribution of $(m+1)$ dimensional subspaces given by the Cartan differential 1-forms

$$
\begin{aligned}
w_{0}^{1} & =d p_{0}^{1}-p_{1}^{1} d x, \ldots, w_{0}^{m}=d p_{0}^{m}-p_{1}^{m} d x \\
w_{1}^{1} & =d p_{1}^{1}-p_{2}^{1} d x, \ldots, w_{1}^{m}=d p_{1}^{m}-p_{2}^{m} d x \\
& \vdots \\
w_{k-1}^{1} & =d p_{k-1}^{1}-p_{k}^{1} d x, \ldots, w_{k-1}^{m}=d p_{k-1}^{m}-p_{k}^{m} d x .
\end{aligned}
$$

In addition, the following statement is true:

Proposition. Let $L$ be a smooth curve in $J^{k}(m)$. It has the form $L_{h}^{(k)}$ for a certain smooth vector-valued function $h(x)=\left(h_{1}(x), \ldots, h_{m}(x)\right)$ if and only if

(A) $L$ is an integral curve of the Cartan distribution:

$$
\left.w_{j}^{i}\right|_{L}=0, \quad i=1, \ldots, m, \quad j=0, \ldots, k-1,
$$

and

(B) $L$ can be "well" projected on the $x$-axis.

2.3. We shall say that a point $a \in \mathcal{E}$ is regular if the tangent subspace $T_{a} \mathcal{E}$ and Cartan subspace $C(a)$ are transversal at this point; otherwise, we shall call it singular.

The restriction of the Cartan distribution to the equation $\mathcal{E}$

$$
l: a \in \mathcal{E} \mapsto l(a)=T_{a} \mathcal{E} \cap C(a),
$$

considered at regular points, determines a distribution of $(m+1-s)$-dimensional subspaces.

In particular, in the case of determinate systems of ordinary differential equations (we shall simply call them differential equations), the distribution $l$ is a directional field.

The following vector field can be chosen as a generator of the directional field $l$ :

$$
Y=\Delta \frac{d}{d x}+\sum_{i, j} \widehat{H}_{i j} \frac{d H_{j}}{d x} \frac{\partial}{\partial p_{k}^{i}} .
$$

Here

$$
\Delta=\operatorname{det}\left\|\frac{\partial H_{j}}{\partial p_{k}^{i}}\right\|
$$


$\left\|\widehat{H}_{i j}\right\|$ is the adjoint of the matrix $\left\|\frac{\partial H_{j}}{\partial p_{k}^{i}}\right\|$, i.e.

$$
\left\|\widehat{H}_{i j}\right\|=\left(\operatorname{det}\left\|\frac{\partial H_{j}}{\partial p_{k}^{i}}\right\|\right)\left\|\frac{\partial H_{j}}{\partial p_{k}^{i}}\right\|^{-1},
$$

and

$$
\frac{d}{d x}=\frac{\partial}{\partial x}+p_{1}^{1} \frac{\partial}{\partial p_{0}^{1}}+\cdots+p_{1}^{m} \frac{\partial}{\partial p_{0}^{m}}+\cdots+p_{k}^{1} \frac{\partial}{\partial p_{k-1}^{1}}+\cdots+p_{k}^{m} \frac{\partial}{\partial p_{k-1}^{m}}
$$

is the total derivation operator. The vector field $Y$ is called characteristic.

Thus, a point $a \in \mathcal{E}$ is singular if and only if the following conditions hold:

$$
\left\{\begin{array}{l}
\frac{d H_{1}}{d x}=0, \ldots, \frac{d H_{m}}{d x}=0 \\
\operatorname{det}\left\|\frac{\partial H_{j}}{\partial p_{k}^{i}}\right\|=0 .
\end{array}\right.
$$

As earlier, the fact that a solution passing through a point $a \in \mathcal{E}$ is regular means that the characteristic vector field $Y$ has a nonzero component along the $x$-axis, i.e.

$$
\Delta=\operatorname{det}\left\|\frac{\partial H_{j}}{\partial p_{k}^{i}}\right\| \neq 0
$$

2.4. Example. Consider the following linear system of differential equations

$$
\left\{\begin{array}{l}
x y_{1}^{\prime}+(1-x) y_{2}^{\prime}+y_{1}=0 \\
(1-x) y_{1}^{\prime}+x y_{2}^{\prime}+y_{2}=0
\end{array}\right.
$$

The corresponding manifold $\mathcal{E}$ in the space $J^{1}(2)$ can be given by the equations

$$
\left\{\begin{array}{l}
x p_{1}^{1}+(1-x) p_{1}^{2}+p_{0}^{1}=0 \\
(1-x) p_{1}^{1}+x p_{1}^{2}+p_{0}^{2}=0
\end{array}\right.
$$

Thus, $\mathcal{E}$ is a 3 -dimensional manifold diffeomorphic to $\mathbb{R}^{3}$. The functions $x, p_{1}^{1}, p_{1}^{2}$ can be chosen as coordinates in $\mathcal{E}$.

Restriction of the Cartan forms

$$
w_{0}^{1}=d p_{0}^{1}-p_{1}^{1} d x \quad \text { and } \quad w_{0}^{2}=d p_{0}^{2}-p_{2}^{1} d x
$$

to $\mathcal{E}$ leads to the following differential 1-forms:

$$
\begin{aligned}
& \tilde{w}_{0}^{1}=\left(p_{1}^{2}-2 p_{1}^{1}\right) d x-x d p_{1}^{1}-(1-x) d p_{1}^{2}, \\
& \tilde{w}_{0}^{2}=\left(p_{1}^{1}-2 p_{1}^{2}\right) d x-(1-x) d p_{1}^{1}-x d p_{1}^{2} .
\end{aligned}
$$


Although singular points $a \in \mathcal{E}$ can be found from relations (11), we shall do it using the fact that the forms $\tilde{w}_{0}^{1}$ and $\tilde{w}_{0}^{2}$ are linearly dependent at singular points.

The following determinants must be zero at all points where $\tilde{w}_{0}^{1}$ and $\tilde{w}_{0}^{2}$ are linearly independent:

$$
\begin{gathered}
\left|\begin{array}{cc}
p_{1}^{2}-2 p_{1}^{1} & -x \\
p_{1}^{1}-2 p_{1}^{2} & x-1
\end{array}\right|=p_{1}^{1}(2-x)+p_{1}^{2}(-1-x), \\
\left|\begin{array}{cc}
p_{1}^{2}-2 p_{1}^{1} & x-1 \\
p_{1}^{1}-2 p_{1}^{2} & -x
\end{array}\right|=p_{1}^{1}(1+x)+p_{1}^{2}(-2+x), \\
\left|\begin{array}{cc}
-x & x-1 \\
x-1 & -x
\end{array}\right|=2 x-1 .
\end{gathered}
$$

Therefore, singular points are points $\left(x, p_{0}^{1}, p_{0}^{2}, p_{1}^{1}, p_{1}^{2}\right)$ such that

$$
x=1 / 2, \quad p_{1}^{1}=p_{1}^{2}, \quad p_{0}^{1}=p_{0}^{2}=-2 p_{1}^{1} .
$$

Thus, the set $\Sigma$ of all singular points of the equation $\mathcal{E}$ is straight line (14), lying in manifold (13).

Since

$$
\operatorname{det}\left\|\frac{\partial H_{j}}{\partial p_{k}^{i}}\right\|=\operatorname{det}\left\|\begin{array}{cc}
x & 1-x \\
1-x & x
\end{array}\right\|=2 x-1,
$$

we see that condition (12) does not hold on the plane $\Pi$ given by the equations

$$
x=1 / 2, \quad p_{0}^{1}=p_{0}^{2}=\frac{1}{2}\left(p_{1}^{1}-p_{1}^{2}\right) .
$$

Therefore, at all points $a \in \Pi \backslash \Sigma$, solutions of our system lose their smoothness and become many-valued.

\section{§3. Completely integrable systems of DIFFERENTIAL EQUATIONS AND DISTRIBUTIONS}

In this section we shall consider the class of first-order partial differential equations which are conceptually close to ordinary differential equations.

3.1. Consider a system of partial differential equations with respect to a vectorvalued function $Y=Y\left(x_{1}, \ldots, x_{n}\right), Y=\left(y_{1}, \ldots, y_{m}\right)$ :

$$
\frac{\partial y_{j}}{\partial x_{i}}=H_{i}^{j}\left(x_{1}, \ldots, x_{n}, y_{1}, \ldots, y_{m}\right)
$$

where $i=1, \ldots, n ; j=1, \ldots, m$. System (1) is called (completely) integrable if its right-hand side satisfies the natural condition that mixed derivatives of the second order be equal:

$$
\frac{\partial^{2} y_{j}}{\partial x_{i} \partial x_{k}}=\frac{\partial^{2} y_{j}}{\partial x_{k} \partial x_{i}} \Leftrightarrow \frac{d}{d x_{k}} H_{i}^{j}=\frac{d}{d x_{i}} H_{k}^{j},
$$


where

$$
\frac{d}{d x_{i}}=\frac{\partial}{\partial x_{i}}+H_{i}^{1} \frac{\partial}{\partial y_{1}}+\cdots+H_{i}^{m} \frac{\partial}{\partial y_{m}}
$$

Conditions (2) can be written more extensively as

$$
\frac{\partial H_{i}^{j}}{\partial x_{k}}+H_{k}^{1} \frac{\partial H_{i}^{j}}{\partial y_{1}}+\cdots+H_{k}^{m} \frac{\partial H_{i}^{j}}{\partial y_{m}}=\frac{\partial H_{k}^{j}}{\partial x_{i}}+H_{i}^{1} \frac{\partial H_{k}^{j}}{\partial y_{1}}+\cdots+H_{i}^{m} \frac{\partial H_{k}^{j}}{\partial y_{m}} .
$$

\subsection{Examples.}

1. If $n=1$, then conditions (4) are obviously satisfied. Thus, systems of ordinary differential equations are completely integrable.

2. The system of differential equations

$$
\left\{\begin{array}{l}
\frac{\partial y}{\partial x_{1}}=x_{2} \\
\frac{\partial y}{\partial x_{2}}=0
\end{array}\right.
$$

with respect to a real-valued function $y=y\left(x_{1}, x_{2}\right)$ is not completely integrable. However, we will see it later that in spite of its apparent ineptitude this system plays a significant part in theory of ordinary differential equations.

3.3. Let us give geometrical interpretation of systems of form (1) in accordance with approaches proposed in $\S \S 1,2$.

Let $J^{0}(n, m)$ denote the space of pairs $(x, y)$, where $x=\left(x_{1}, \ldots, x_{n}\right) \in \mathbb{R}^{n}$ and $y \in \mathbb{R}^{m}$. Each solution $y=h(x)$ of system (1) determines the $n$-dimensional surface in $J^{0}(n, m)$ :

$$
L_{h}=\{(x, y) \mid y=h(x)\},
$$

which is the graph of the function $h(x)$.

According to the terminology introduced in the previous sections, we say that $J^{0}(n, m)$ is the space of 0 -jets of vector-valued functions. For an $a \in \mathbb{R}^{n}$, we shall say that the pair

$$
[h]_{a}^{1}=\left[a \text {; the tangent plane to } L_{h} \text { at the point }(a, h(a))\right]
$$

is the 1-jet of the function $h(x)$ at the point a.

A 1 -jet $[h]_{a}^{1}$ can analytically be given by the value $h(a)$ together with the values of all possible first-order partial derivatives:

$$
\frac{\partial h_{j}}{\partial x_{i}}(a), \quad i=1, \ldots, n ; j=1, \ldots, m
$$

Therefore, from a geometrical standpoint, simultaneous differential equations (1) determine a field of $n$-dimensional subspaces in $J^{0}(n, m)$ :

$$
E:(x, y) \mapsto E(x, y)
$$


which is called a distribution or differential system.

Conversely, let $E$ be an $n$-dimensional distribution in $J^{0}(n, m)$ such that the projection of each subspace $E(x, y)$ into the space tangent to the $x$-space at $x$ is nonsingular. Then $E$ determines a system of differential equations of form (1). Indeed, the distribution $E$ can be given by choosing a basis in each $E(x, y)$. Moreover, this basis can always by chosen so that its projection into the space tangent to the $x$-space is the standard basis

$$
\frac{\partial}{\partial x_{1}}, \ldots, \frac{\partial}{\partial x_{n}}
$$

Thus, as a basis of the distribution $E$ we can choose vector fields of the form:

$$
\left\{\begin{array}{l}
A_{1}=\frac{\partial}{\partial x_{1}}+H_{1}^{1} \frac{\partial}{\partial y_{1}}+\cdots+H_{1}^{m} \frac{\partial}{\partial y_{m}} \\
\vdots \\
A_{n}=\frac{\partial}{\partial x_{n}}+H_{n}^{1} \frac{\partial}{\partial y_{1}}+\cdots+H_{n}^{m} \frac{\partial}{\partial y_{m}}
\end{array}\right.
$$

Conditions for vector fields (6) to be tangent to the graph $L_{h}$ of a function $y=h(x)$ are as follows:

$$
\begin{aligned}
& \frac{\partial h_{1}}{\partial x_{1}}=H_{1}^{1}, \ldots, \frac{\partial h_{1}}{\partial x_{n}}=H_{n}^{1}, \\
& \vdots \\
& \frac{\partial h_{m}}{\partial x_{1}}=H_{1}^{m}, \ldots, \frac{\partial h_{m}}{\partial x_{n}}=H_{n}^{m},
\end{aligned}
$$

which is equivalent to simultaneous system of differential equations (1).

It is easy to verify that in this case conditions (4) are equivalent to the condition that vector fields (6) commute with each other:

$$
\left[A_{i}, A_{k}\right]=0
$$

for all $i, k=1, \ldots, n$.

Let us write vector fields (6) as

$$
A_{1}=\frac{\partial}{\partial x_{1}}+H_{1}, \ldots, A_{n}=\frac{\partial}{\partial x_{n}}+H_{n}
$$

where

$$
H_{i}=\sum_{j=1}^{m} H_{i}^{j} \frac{\partial}{\partial y_{j}} .
$$

Then conditions (7) take the form of "zero curvature conditions":

$$
\frac{\partial H_{i}}{\partial x_{k}}-\frac{\partial H_{k}}{\partial x_{i}}+\left[H_{i}, H_{k}\right]=0
$$


3.4. Distributions can also be defined dually, by giving a set of differential 1-forms annihilating a given distribution. In our case it can be easily seen that as a basis for the space of forms of this sort we can choose the following ones:

$$
\begin{aligned}
& \theta_{1}=d y_{1}-H_{1}^{1} d x_{1}-\cdots-H_{n}^{1} d x_{n}, \\
& \vdots \\
& \theta_{m}=d y_{m}-H_{1}^{m} d x_{1}-\cdots-H_{n}^{m} d x_{n} .
\end{aligned}
$$

It is easy to check that the restrictions of forms (10) to the graph $L_{h}$ of a function $y=h(x)$ vanish if and only if $y=h(x)$ is a solution of system (1). This is exactly what we mean when we write system (1) in the form of the Pfaff system:

$$
\theta_{1}=0, \ldots, \theta_{m}=0
$$

We shall say that system (11) is completely integrable if so is the corresponding system of form (1).

3.5. Proposition. Pfaff system (11) is completely integrable if and only if

$$
d \theta_{j} \wedge \theta_{1} \wedge \cdots \wedge \theta_{m}=0
$$

for all $j=1, \ldots, m$.

Proof. Conditions (12) are equivalent to the condition that each of the differential 2 -forms $d \theta_{1}, \ldots, d \theta_{m}$ be a linear combination of 1 -forms $\theta_{1}, \ldots, \theta_{m}$, i.e.

$$
d \theta_{j}=\gamma_{j_{1}} \wedge \theta_{1}+\cdots+\gamma_{j_{m}} \wedge \theta_{m}
$$

for all $j=1, \ldots, m$. Conditions (13) are in their turn equivalent to the condition that these 2 -forms vanish on each subspace $E(x, y)$ of our distribution. It remains to make use of the following well known formula

$$
d \theta_{j}\left(v_{1}, v_{2}\right)=v_{1}\left(\theta_{j}\left(v_{2}\right)\right)-v_{2}\left(\theta_{j}\left(v_{1}\right)\right)-\theta_{j}\left(\left[v_{1}, v_{2}\right]\right) .
$$

It follows that for arbitrary vector fields $v_{1}$ and $v_{2}$ lying in the distribution, their commutator $\left[v_{1}, v_{2}\right]$ also belongs to the distribution (i.e. condition (9) holds) if and only if $d \theta_{j}$ vanishes on the distribution.

\subsection{Examples.}

1. Consider the following system of ordinary differential equations:

$$
\frac{\partial y_{j}}{\partial x}=H^{j}(x, y)
$$


where $x \in \mathbb{R}$ and $j=1, \ldots, m$. The corresponding distribution is a directional field $(x, y) \mapsto E(x, y)$ such that $E(x, y)$ is the straight line spanned by the vector

$$
A=\frac{\partial}{\partial x}+H^{1} \frac{\partial}{\partial y_{1}}+\cdots+H^{m} \frac{\partial}{\partial y_{m}}
$$

This is the classical correspondence between vector fields and differential equations. The corresponding Pfaff system has the form

$$
\theta_{1}=d y_{1}-H^{1} d x=0, \ldots, \theta_{m}=d y_{m}-H^{m} d x=0 .
$$

2. Recall the system of differential equations from example 3.2.(2). As a basis of the corresponding distribution we choose

$$
A_{1}=\frac{\partial}{\partial x_{1}}+x_{2} \frac{\partial}{\partial y} \text { and } A_{2}=\frac{\partial}{\partial x_{2}}
$$

The corresponding differential 1-form has the form

$$
\theta=d y-x_{2} d x_{1}
$$

Note that we have obtained nothing but the Cartan distribution $\left(y \leftrightarrow p_{0}, x_{2} \leftrightarrow\right.$ $\left.p_{1}, x_{1} \leftrightarrow x_{0}\right)$. It is called the standard contact distribution in $\mathbb{R}^{3}\left(=J^{1}\right)$.

\section{$\S 4$. Distributions}

As we saw in previous sections, the concept of a distribution is one of the most useful concepts in theory of differential equations. In this section we shall give basic definitions and results connected with distributions.

4.1. Let $M$ be a smooth manifold of dimension $m+n$. A distribution (or differential system) on $M$ is a field of $m$-dimensional subspaces:

$$
E: x \in M \mapsto E(x) \subset \mathrm{T}_{x} V
$$

such that $E$ is a smooth mapping of $M$.

Here the fact that $E$ is smooth means that for any $x_{0} \in M$ there exist a neighborhood $\mathcal{O}$ of $x_{0}$ and $m$ smooth vector fields $A_{1}, \ldots, A_{m}$ defined on $\mathcal{O}$ such that at each point $x \in \mathcal{O}$ vectors $A_{1}(x), \ldots, A_{m}(x)$ form a basis of the subspace $E(x)$.

The number $m$ is called the dimension of the distribution $E$ and is denoted by $\operatorname{dim} E$. The number $n=\operatorname{dim} M-\operatorname{dim} E$ is called the codimension of $E$ and is denoted by $\operatorname{codim} E$.

We say that a vector field $A$ on $M$ lies in a distribution $E$ if $A(x) \in E(x)$ for all $x \in M$.

In the sequel, in order to locally define a distribution $E$, we shall use two methods. Firstly, $E$ can be defined by a set of $m$ vector fields $A_{1}, \ldots, A_{m}$ such that the vectors 
$A_{1}(x), \ldots, A_{m}(x)$ form a basis of the subspace $E(x)$ for all points $x$ of some neighborhood $\mathcal{O}$. Secondly (the dual method), it can be defined by a set of $n$ differential 1 -forms $\theta_{1}, \ldots, \theta_{n}$ linearly independent at every point $x \in \mathcal{O}$ :

$$
E(x)=\left\{v \in \mathrm{T}_{x} M \mid \theta_{1, x}(v)=0, \ldots, \theta_{n, x}(v)=0\right\} .
$$

In the first case we shall denote $E$ by $\mathcal{F}\left(A_{1}, \ldots, A_{m}\right)$, while in the second case by $\mathcal{F}\left(\theta_{1}, \ldots, \theta_{n}\right)$.

4.2. A submanifold $L \subset M$ is called an integral manifold of a distribution $E$ if

$$
\mathrm{T}_{x}(L) \subset E(x)
$$

for all $x \in L$.

This definition implies that the dimension of an integral manifold cannot exceed the dimension of the distribution.

A distribution $E$ is said to be completely integrable (or involutory) if for any point $x \in M$ there exists an integral manifold $L$ such that $x \in L$ and $\operatorname{dim} L=\operatorname{dim} E$.

4.3. Example. Let $(x, y, z)$ be coordinates in $\mathbb{R}^{3}\left(=J^{1}\right)$. Consider the distribution $E=\mathcal{F}(\omega)$ in $\mathbb{R}^{3}$ given by the differential 1 -form

$$
\omega=d z-y d x .
$$

Each 1-dimensional integral manifold of this distribution can be written as a curve of the form

$$
L=\{x=x(t), y=y(t), z=z(t)\},
$$

where $t \in \mathbb{R}$ is a parameter on $L$.

For all $a \in L$ we have $\mathrm{T}_{a}(L) \subset E(a)$. This means that the form $\omega$ vanishes on the tangent vector $v=(\dot{x}(t), \dot{y}(t), \dot{z}(t))$ and therefore

$$
\omega(v)=\dot{z}(t)-y(t) \dot{x}(t)=0 .
$$

Thus, if we put

$$
\dot{z}(t)=\int y(t) \dot{x}(t) d t
$$

for arbitrary functions $x(t)$ and $y(t)$, we shall obtain a 1-dimensional integral manifold (an integral curve) of the distribution $E$.

Let us show that $E$ has no 2-dimensional integral manifolds. First we shall prove it by direct calculations. So, assume that $L$ is a 2-dimensional integral surface of $E$ and let

$$
x=x(s, t), \quad y=y(s, t), \quad z=z(s, t)
$$

be its parametric representation, where $s, t \in \mathbb{R}$ are parameters and the rank of the Jacobian matrix

$$
J=\left(\begin{array}{lll}
\frac{\partial x}{\partial s} & \frac{\partial y}{\partial s} & \frac{\partial z}{\partial s} \\
\frac{\partial x}{\partial t} & \frac{\partial y}{\partial t} & \frac{\partial z}{\partial t}
\end{array}\right)
$$


equals 2 .

From the condition that $L$ be integral it follows that the restriction of the form $\omega$ to $L$ equals zero. Thus

$$
0=\left.\omega\right|_{L}=d z(s, t)-y(s, t) d x(s, t)=\left(\frac{\partial z}{\partial s}-y \frac{\partial x}{\partial s}\right) d s+\left(\frac{\partial z}{\partial t}-y \frac{\partial x}{\partial t}\right) d t=0
$$

and therefore

$$
\frac{\partial z}{\partial s}=y \frac{\partial x}{\partial s}, \quad \frac{\partial z}{\partial t}=y \frac{\partial x}{\partial t}
$$

Using

$$
\frac{\partial^{2} z}{\partial s \partial t}=\frac{\partial^{2} z}{\partial t \partial s}
$$

we obtain

$$
\frac{\partial y}{\partial t} \frac{\partial x}{\partial s}-\frac{\partial y}{\partial s} \frac{\partial x}{\partial t}=0
$$

Now, multiplying equations (3) by $\frac{\partial y}{\partial t}$ and $\frac{\partial y}{\partial s}$ respectively, we obtain

$$
\frac{\partial z}{\partial s} \frac{\partial y}{\partial t}-\frac{\partial z}{\partial t} \frac{\partial y}{\partial s}=0
$$

Conditions (4) and (5) contradict the assumption that the rank of the matrix $J$ equals 2. Thus, the distribution $E$ has no 2-dimensional integral manifolds and therefore is not completely integrable.

This can be proved in a different way, using the fact that the restriction of the differential 2-form $d \omega$ to the tangent plane $\mathrm{T}_{a} L$ must also be zero:

$$
\left.(d \omega)\right|_{L}=d\left(\left.\omega\right|_{L}\right)=0 \text {. }
$$

But, by reasons of dimension, the plane $\mathrm{T}_{a} L$ coincides with $E(a)$. Since the 2 -form $d \omega=-d y \wedge d x$ is non-degenerate on $E(a)$, we see that $d \omega$ does not vanish on $\mathrm{T}_{a} L$.

This example, which is typical in some sense, shows that every distribution $E$ may have integral manifolds of dimension less then $\operatorname{dim} E$ (for instance, integral curves). However, not all distributions are completely integrable, i.e. may have distributions of the maximal dimension equal to $\operatorname{dim} E$.

Let us find conditions for a distribution $E$ to be completely integrable. Assume that $E$ is given by differential 1 -forms $\theta_{1}, \ldots, \theta_{m}$, i.e. $E=\mathcal{F}\left(\theta_{1}, \ldots, \theta_{m}\right)$, and let $L \subset M$ be an integral submanifold such that $\operatorname{dim} L=\operatorname{dim} E$. Then

$$
\left.\left(d \theta_{j}\right)\right|_{L}=d\left(\left.\theta_{j}\right|_{L}\right)=0
$$

for all $j=1, \ldots, m$.

Thus, for any point $a \in L$, the exterior differential 2 -forms $d \theta_{1, a}, \ldots, d \theta_{m, a}$ vanish on the subspace

$$
\mathrm{T}_{a} L=E(a)=\left\{v \in \mathrm{T}_{a} M \mid \theta_{1, a}(v)=0, \ldots, \theta_{m, a}(v)=0\right\} .
$$


Therefore, they can be written as

$$
d \theta_{j, a}=\lambda_{j} 1(a) \wedge \theta_{1, a}+\cdots+\lambda_{j m}(a) \wedge \theta_{m, a}(a)
$$

where $j=1, \ldots, m$ and $\lambda_{j 1}(a), \ldots, \lambda_{j m}(a) \in \mathrm{T}_{a}^{*} M$ are some covectors.

If $E$ is a completely integrable distribution, then relation (6) holds for all points of $M$ and therefore the decomposition

$$
d \theta_{j, a}=\lambda_{j 1} \wedge \theta_{1}+\cdots+\lambda_{j m} \wedge \theta_{m}
$$

$j=1, \ldots, m$, holds for certain differential 1 -forms $\lambda_{j i}$. Conditions (7) can be rewritten without using $\lambda_{j i}$ :

$$
d \theta_{j} \wedge \theta_{1} \wedge \cdots \wedge \theta_{m}=0
$$

for all $j=1, \ldots, m$.

Now assume that $E$ is given by linearly independent vector fields $A_{1}, \ldots, A_{n}$, i.e. $E=\mathcal{F}\left(A_{1}, \ldots, A_{n}\right)$. The formula

$$
d \theta(X, Y)=X(\theta(Y))-Y(\theta(X))-\theta([X, Y])
$$

shows that the 2-forms $d \theta_{j}$ vanish on the distribution $E$ if and only if

$$
d \theta_{j}\left(A_{k}, A_{l}\right)=0
$$

for all $k, l=1, \ldots, n$. But (9) implies that this is true if and only if the commutators $\left[A_{k}, A_{l}\right]$ also lie in $E$, so that

$$
\left[A_{k}, A_{l}\right]=\sum_{i=1}^{n} c_{k l}^{i} A_{i}
$$

for all $k, l=1, \ldots, n$, and some smooth functions $c_{k l}^{i}$.

The following theorem shows that each of conditions $(7),(8),(10)$ are sufficient for $E$ to be completely integrable.

\subsection{Frobenius' theorem.}

1st variant. A distribution $E=\mathcal{F}\left(\theta_{1}, \ldots, \theta_{m}\right)$ is completely integrable if and only if conditions (7) (or equivalent conditions (8)) are satisfied.

2nd variant. A distribution $E=\mathcal{F}\left(A_{1}, \ldots, A_{n}\right)$ is completely integrable if and only if conditions (10) are satisfied.

We shall first prove the following

Lemma. Let $E=\mathcal{F}\left(\theta_{1}, \ldots, \theta_{m}\right)$ be a distribution satisfying conditions (7) and (8). Suppose $A$ is a vector field lying in $E$ and $\left\{F_{t}\right\}$ is the one-parameter group of translations along the vector field $A$. Then for all $t \in \mathbb{R}$ the differential 2-forms 
$F_{t}^{*}\left(\theta_{1}\right), \ldots, F_{t}^{*}\left(\theta_{m}\right)$ vanish on $E$ and therefore are linear combinations of the forms $\theta_{1}, \ldots, \theta_{m}$.

Proof of the lemma. Consider the differential $(m+1)$-forms

$$
\Theta_{j}(t)=F_{t}^{*}\left(\theta_{j}\right) \wedge \theta_{1} \wedge \cdots \wedge \theta_{m}
$$

$j=1, \ldots, m$. Let us find their derivatives with respect to the parameter $t$. We have

$$
\frac{d \Theta_{j}}{d t}=\frac{d}{d t} F_{t}^{*}\left(\theta_{j}\right) \wedge \theta_{1} \wedge \cdots \wedge \theta_{m}=F_{t}^{*}\left(L_{A}\left(\theta_{j}\right)\right) \wedge \theta_{1} \wedge \cdots \wedge \theta_{m} .
$$

But

$$
\left.\left.\left.L_{A}\left(\theta_{j}\right)=A\right\lrcorner d \theta_{j}+d(A\lrcorner \theta_{j}\right)=A\right\lrcorner d \theta_{j}=\lambda_{j 1}(A) \theta_{1}+\cdots+\lambda_{j m}(A) \theta_{m} .
$$

Therefore,

$$
\frac{d \Theta_{j}}{d t}=\Lambda_{j 1}(t) \Theta_{1}+\cdots+\Lambda_{j m}(t) \Theta_{m}
$$

where

$$
\Lambda_{j k}(t)=F_{t}^{*}\left(\lambda_{j k}(A)\right) .
$$

Thus the forms $\Theta_{1}(t), \ldots, \Theta_{m}(t)$ satisfy simultaneous linear ordinary differential equations (11). Since $\Theta_{j}(0)=0$ for all $j=1, \ldots, m$, from the unique solution theorem it follows that $\Theta_{j}(t) \equiv 0$ for all $j=1, \ldots, m$ and $t \in \mathbb{R}$. This concludes the proof of the lemma.

Proof of the theorem. Let $L \subset M$ be an integral manifold of the distribution $E$ and $A$ a vector field such that $A$ lies in $E$ and is not tangent to $L$. Since by the lemma we have

$$
\left.\theta_{j}\right|_{F_{t}(L)}=\left.F_{t}^{*}\left(\theta_{j}\right)\right|_{L}=0
$$

we see that the submanifolds $L_{t}=F_{t}(L)$ are also integral manifolds of $E$. In addition, for sufficiently small $t$ the set

$$
\widehat{L}=\bigcup_{t} L_{t}
$$

is a smooth submanifold in $M$ (see Fig. 10).

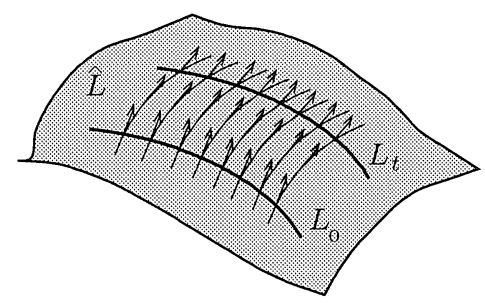

Fig. 10 
For a point $a \in L_{t}$ consider the tangent space $\mathrm{T}_{a}(\widehat{L})$. This space is the sum of the space $T_{a}\left(L_{t}\right) \subset E(a)$ and the straight line spanned by the vector $A_{a} \in E(a)$. Therefore, $L$ is an integral manifold of the distribution $E$.

Thus, if $\operatorname{dim} L<\operatorname{dim} E$, then we can construct an integral manifold of dimension $1+\operatorname{dim} L$. Starting from, for instance, the trivial 0-dimensional manifold, we can construct an integral manifold of dimension equal to $\operatorname{dim} E$.

4.5. Example. Distributions of codimension 1.

A distribution of codimension 1 can be given (locally) by one differential 1-form $\theta$. This distribution is completely integrable if and only if

$$
d \theta \wedge \theta=0
$$

For example, let $M=\mathbb{R}^{2}$ and

$$
\theta=A(x, y, z) d x+B(x, y, z) d y+C(x, y, z) d z .
$$

Then

$$
d \theta \wedge \theta=\left[A\left(\frac{\partial B}{\partial z}-\frac{\partial C}{\partial y}\right)-B\left(\frac{\partial A}{\partial z}-\frac{\partial C}{\partial x}\right)+C\left(\frac{\partial B}{\partial x}-\frac{\partial A}{\partial y}\right)\right] d x \wedge d y \wedge d z .
$$

Thus, the distribution $\mathcal{F}(\theta)$ is completely integrable if and only if

$$
A\left(\frac{\partial B}{\partial z}-\frac{\partial C}{\partial y}\right)-B\left(\frac{\partial A}{\partial z}-\frac{\partial C}{\partial x}\right)+C\left(\frac{\partial B}{\partial x}-\frac{\partial A}{\partial y}\right)=0
$$

4.6. Remark. There is a connection between integral manifolds of distributions and solutions of systems of differential equations considered in $\S 3$. Let $E$ be a distribution on $M$. We can choose coordinates $x_{1}, \ldots, x_{n}, y_{1}, \ldots, y_{m}$ in some neighborhood $\mathcal{O}$ of a point $a \in M$ so that for any $x \in \mathcal{O}$ the restrictions of the differential 1-forms $d x_{1}, \ldots, d x_{n}$ to the subspace $E(x)$ are linearly independent. The forms $d y_{1}, \ldots, d y_{m}$ considered on the subspaces $E(x), x \in \mathcal{O}$, are linear combinations of the forms $d x_{1}, \ldots, d x_{n}$. Therefore, $E$ can be given by forms $\theta_{1}, \ldots, \theta_{m}$ of the form:

$$
\left\{\begin{array}{l}
\theta_{1}=d y_{1}-H_{1}^{1}(x, y) d x_{1}-\cdots-H_{n}^{1}(x, y) d x_{n}, \\
\vdots \\
\theta_{m}=d y_{m}-H_{1}^{m}(x, y) d x_{1}-\cdots-H_{n}^{m}(x, y) d x_{n} .
\end{array}\right.
$$

Now let $L$ be an integral manifold of $E$ passing through a point $a \in M$ and suppose that $\operatorname{dim} L=\operatorname{dim} E$. Then in some neighborhood of $a, L$ has the form of the graph $L_{h}$ for some vector-valued function $y=h(x)$. Conditions for $L$ to be integrable:

$$
\left.\theta_{j}\right|_{L_{h}}=0
$$

are exactly differential equations (1) from $\S 3$, while conditions of Frobenius' theorem are exactly conditions (4) from $§ 3$. 


\section{$\S 5$. JETS OF CURVES}

In final sections of this chapter we shall give an invariant interpretation for basic concepts of geometrical theory of ordinary differential equations on manifolds. This will enable us to use language free of choice of local coordinate systems and to introduce some new and useful structures in spaces of jets.

5.1. Let $N$ be a smooth manifold of dimension $n+1$. A curve in $M$ is a 1dimensional submanifold $L \subset N$. Suppose that $N$ can be written as a direct product of a smooth manifold $M$ and the set of real numbers $\mathbb{R}: \mathbb{N}=M \times \mathbb{R}$ so that the projection of $L$ on $\mathbb{R}$ is a diffeomorphism of $L$ onto some open domain $\mathcal{O} \subset \mathbb{R}$. Then $L$ is called a parametrized curve in $M$. (See Fig. 11 and 12.)

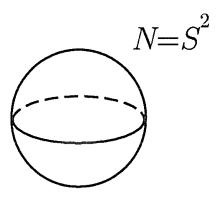

Fig. 11

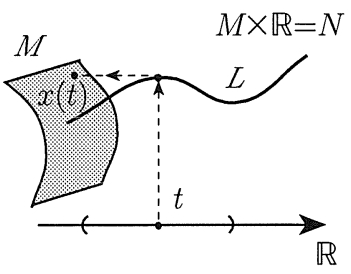

Fig. 12

The submanifold $L$ determines the smooth mapping

$$
x: \mathcal{O} \rightarrow M
$$

that takes a point $t \in \mathcal{O}$ into $x(t) \in M$ so that $(x(t), t) \in L$ (see Fig. 12). In other words, $L$ is the graph of the mapping $x$.

5.2. Definition. Let $L_{1}$ and $L_{2}$ be submanifolds of $N, k$ a natural number, and $a \in L_{1} \cap L_{2}$. We shall say that the submanifolds $L_{1}$ and $L_{2}$ have contact (or tangency) of order $k$ at the point $a$ if for any smooth function $f \in C^{\infty}(N)$ vanishing on $L_{1}$, the function $\left.f\right|_{L_{2}}$ has zero of order $\geqslant k+1$ at the point $a \in L_{2}$.

Example. Let $N$ be the plane with coordinates $(t, q)$. Consider the curves $L_{1}$ and $L_{2}$ given by

$$
\begin{aligned}
& L_{1}=\{(t, q) \mid q=0\}, \\
& L_{2}=\{(t, q) \mid q=h(t)\},
\end{aligned}
$$

where $h(t)$ is a smooth function vanishing at the point $t=0$ (see Fig. 13). 


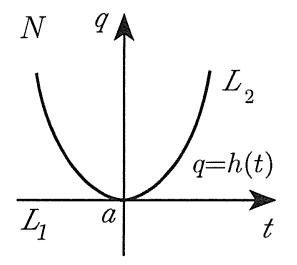

Fig. 13

All functions vanishing on $L_{1}$ have the form $q \cdot g(t, q)$ for some $g \in C^{\infty}(N)$. Therefore, in order to find the order of tangency of $L_{1}$ and $L_{2}$, it is sufficient to put $f=q$. But $\left.q\right|_{L_{2}}=h(t)$. Thus, the order of tangency is determined by the order of zero of the function $h(t)$.

5.3. A condition for parametrized curves

$$
x: \mathcal{O} \rightarrow M \text { and } y: \mathcal{O}^{\prime} \rightarrow M
$$

to have contact of order $k$ at a point $t_{0} \in \mathcal{O} \cap \mathcal{O}^{\prime}$ can be reformulated as follows:

Definition. The parametrized curves $x(t)$ and $y(t)$ have contact of order $k$ at the point $t_{0} \in \mathcal{O} \cap \mathcal{O}^{\prime}$ if for any smooth function $f \in C^{\infty}(M)$, the function

$$
\begin{gathered}
x^{*}(f)-y^{*}(f): \mathcal{O} \cap \mathcal{O}^{\prime} \rightarrow \mathbb{R}, \\
\left(x^{*}(f)-y^{*}(f)\right)(t)=f(x(t))-f(y(t))
\end{gathered}
$$

has zero of order $\geqslant k+1$ at $t_{0}$.

Let $t_{0} \in \mathbb{R}$. By $\mu_{t_{0}}^{k}$ denote the ideal in $C^{\infty}(\mathbb{R})$ consisting of all smooth functions that have zero of order $k$ at the point $t_{0}$. Then the curves $x(t)$ and $y(t)$ have contact of order $k$ at $t_{0}$ if and only if

$$
x^{*}(f)-y^{*}(f) \in \mu_{t_{0}}^{k+1}
$$

for all functions $f \in C^{\infty}(M)$.

5.4. Note that the definition above has local nature. If functions $h_{1}$ and $h_{2}$ coincide in some neighborhood of a point $t_{0} \in \mathbb{R}$ and $h_{1} \in \mu_{t_{0}}^{k}$, then $h_{2} \in \mu_{t_{0}}^{k}$. Therefore, local coordinates can be chosen as functions $f$ in the definition above. Suppose that $u^{1}, \ldots, u^{n}$ are local coordinates in a neighborhood of the point $x\left(t_{0}\right) \in M$ and the curves $x(t)$ and $y(t)$ have the following form in these coordinates:

$$
x(t): u^{1}=x^{1}(t), \ldots, u^{n}=x^{n}(t)
$$




$$
y(t): u^{1}=y^{1}(t), \ldots, u^{n}=y^{n}(t) .
$$

Then the curves $x(t)$ and $y(t)$ have contact of order $k$ at the point $t_{0} \in \mathbb{R}$ if and only if

$$
\left(x^{*}\left(u^{j}\right)-y^{*}\left(u^{j}\right)\right)(t)=x^{j}(t)-y^{j}(t) \in \mu_{t_{0}}^{k+1}
$$

for all $j=1, \ldots, n$. In other words, vectors

$$
\begin{aligned}
& \left(x^{1}\left(t_{0}\right), \ldots, x^{n}\left(t_{0}\right),\left(x^{1}\right)^{\prime}\left(t_{0}\right), \ldots,\left(x^{n}\right)^{\prime}\left(t_{0}\right), \ldots,\left(x^{1}\right)^{(k)}\left(t_{0}\right), \ldots,\left(x^{n}\right)^{(k)}\left(t_{0}\right)\right) \\
& \left(y^{1}\left(t_{0}\right), \ldots, y^{n}\left(t_{0}\right),\left(y^{1}\right)^{\prime}\left(t_{0}\right), \ldots,\left(y^{n}\right)^{\prime}\left(t_{0}\right), \ldots,\left(y^{1}\right)^{(k)}\left(t_{0}\right), \ldots,\left(y^{n}\right)^{(k)}\left(t_{0}\right)\right)
\end{aligned}
$$

coincide.

\subsection{Definition.}

1) Curves $L_{1}, L_{2} \subset N$ are said to be $k$-equivalent at a point $a \in L_{1} \cap L_{2}$ if they have contact of order $\geqslant k$ at this point. The $k$-equivalence class determined by a curve $L$ at a point $a \in L$ is called the $k$-jet of the curve $L$ at the point $a$ and is denoted by $[L]_{a}^{k}$.

2) Parametrized curves $x: \mathcal{O} \rightarrow M$ and $y: \mathcal{O}^{\prime} \rightarrow M$ are said to be $k$-equivalent at a point $t_{0} \in \mathcal{O}$ if they have contact of order $\geqslant k-1$ at this point. The $k$-equivalence class determined by a curve $x$ at a point $t_{0} \in \mathcal{O}$ is called the $k$-jet of the parametrized curve $x$ at the point $t_{0}$ and is denoted by $[x]_{t_{0}}^{k}$.

5.6. By $N_{a}^{k}$ denote the set of all $k$-jets of curves on $N$ at a fixed point $a \in N$ and by $N^{k}$ denote the set of all $k$-jets:

$$
N^{k}=\bigcup_{a \in N} N_{a}^{k}
$$

Now let $J_{\left(t_{0}, a\right)}^{k}(\mathbb{R}, M)$ denote the set of all $k$-jets $[x]_{t_{0}}^{k}$ of parametrized curves $x$ on $M$ at a fixed $t_{0}$ such that $a=x\left(t_{0}\right)$. By $J^{k}(\mathbb{R}, M)$ denote the set of all $k$-jets of parametrized curves on $M$ :

$$
J^{k}(\mathbb{R}, M)=\bigcup_{\left(t_{0}, a\right)} J_{\left(t_{0}, a\right)}^{k}(\mathbb{R}, M)
$$

Let us consider these spaces for small numbers $k$. If $k=0$, then obviously

$$
N^{0}=N \text { and } J^{0}(\mathbb{R}, M)=\mathbb{R} \times M .
$$

If $k=1$, then each 1 -jet $[x]_{t_{0}}^{1}$ can be identified with the tangent vector $\dot{x}\left(t_{0}\right)$. Thus,

$$
J_{\left(t_{0}, a\right)}^{1}(\mathbb{R}, M)=\mathrm{T}_{a} M
$$

and therefore

$$
J^{1}(\mathbb{R}, M)=\mathbb{R} \times \mathrm{T} M
$$


Correspondingly, each 1 -jet $[L]_{a}^{1}$ can be identified with a 1-dimensional subspace of the tangent space $\mathrm{T}_{a} N$. Thus

$$
N_{a}^{1}=\mathbb{P}\left(\mathrm{T}_{a} N\right)
$$

is the projectivisation of the tangent space $T_{a} N$, and

$$
N^{1}=\mathbb{P}(\mathrm{T} N)
$$

is the projectivisation of the tangent bundle $\mathrm{T} N$.

5.7. In order to describe the spaces of $k$-jets of curves for $k \geqslant 2$, consider the natural projections

$$
\begin{aligned}
\pi_{k, s}: J^{k}(\mathbb{R}, M) & \rightarrow J^{s}(\mathbb{R}, M), \\
\pi_{k, s}: N^{k} & \rightarrow N^{s}
\end{aligned}
$$

generated by reduction of $k$-jets of curves to $s$-jets, $k \geqslant s$. For example,

$$
\pi_{k, s}\left([L]_{a}^{k}\right)=[L]_{a}^{s} .
$$

The spaces $J^{k}(\mathbb{R}, M)$ and $N^{k}$, for $k=0,1,2, \ldots$, can be naturally turned into smooth manifolds. We shall begin with the space $J^{k}(\mathbb{R}, M)$. Let $u^{1}, \ldots, u^{n}$ be local coordinates in some neighborhood $U$ of a point $a \in M$. These coordinates determine the local coordinates

$$
t, u^{1}, \ldots, u^{n}, p_{1}^{1}, \ldots, p_{1}^{n}, \ldots, p_{k}^{1}, \ldots, p_{k}^{n}
$$

in the neighborhood $\pi_{k, 0}^{-1}(\mathcal{O} \times U)$ of a point $[x]_{t_{0}}^{k} \in J^{k}(\mathbb{R}, M)$. Here $\mathcal{O}$ is some interval containing $t_{0}$ and the coordinate functions $p_{j}^{i}$ are defined by

$$
p_{j}^{i}\left([x]_{t}^{k}\right)=\left(x^{i}\right)^{(j)}(t) .
$$

The rules for differentiation of composite functions show that the functions

$$
t, u^{j}, p_{j}^{i}, \quad i=1, \ldots, n ; j=1, \ldots, k
$$

give a local coordinate system on the set $J^{k}(\mathbb{R}, M)$, thereby turning $J^{k}(\mathbb{R}, M)$ into a smooth manifold of dimension $n(k+1)+1$.

In order to supply the space $N^{k}$ with a structure of a smooth manifold, let us note that the manifold $N$ can be locally represented as the direct product $M^{\prime} \times \mathbb{R}$. The embedding

$$
J^{k}\left(\mathbb{R}, M^{\prime}\right) \hookrightarrow N^{k},
$$

corresponding to this representation, together with the smooth manifold structure in $J^{k}\left(\mathbb{R}, M^{\prime}\right)$ just described determine an atlas on the manifold $N^{k}$.

Projections $\pi_{k, s}$ can be regarded as smooth fibrations with respect to the smooth manifold structure described. Therefore, the manifold of $k$-jets can be imagined if we successively describe fibres of the fibrations $\pi_{n, n-1}$ for $n=2,3, \ldots, k$. With this in mind, we shall digress for a while and consider a new object. 


\section{§6. Differential groups}

6.1. Let $G$ denote the group of all diffeomorphisms of a manifold $M$. We shall identify every diffeomorphism $\varphi \in G$ with its graph $\Gamma_{\varphi} \subset M \times M$, where

$$
\Gamma_{\varphi}=\{(x, y) \in M \times M \mid y=\varphi(x)\}
$$

Definition. Let $\varphi, \psi \in G$ be diffeomorphisms of $M$ such that $\varphi(a)=\psi(a)=b$ for some points $a, b \in M$. We shall say that $\varphi$ and $\psi$ have contact of order $k$ at the point $a$ if the submanifolds $\Gamma_{\varphi}$ and $\Gamma_{\psi}$ have contact of order $k$ at the point $(a, b) \in \Gamma_{\varphi} \cap \Gamma_{\psi}$.

In other words, diffeomorphisms $\varphi$ and $\psi$ of $M$ have contact of order $k$ at a point $a \in M$ if $\varphi(a)=\psi(a)$ and for any smooth function $f \in C^{\infty}(M)$ the following condition holds:

$$
\varphi^{*}(f)-\psi^{*}(f) \in \mu_{a}^{k+1} .
$$

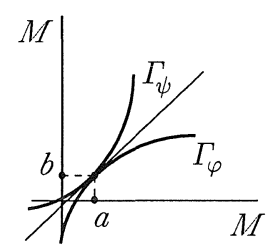

Fig. 14

6.2. Definition. Diffeomorphisms $\varphi, \psi \in G$ are said to be $k$-equivalent at a point $a \in M$ if they have contact of order $\geqslant k$ at this point. The $k$-equivalence class determined by $\varphi$ at $a \in M$ is called the $k$-jet of the diffeomorphism $\varphi$ at the point $a$ and is denoted by $[\varphi]_{a}^{k}$.

6.3. The definition of contact of two diffeomorphisms has local nature. Therefore, it is sufficient to check condition (1) only for coordinate functions. Let $x_{1}, \ldots, x_{n}$ be local coordinates in a neighborhood of the point $a$ and $y_{1}, \ldots, y_{n}$ local coordinates in a neighborhood of the point $b=\varphi(a)$. In these coordinates the diffeomorphisms $\varphi$ and $\psi$ have the form

$$
\varphi=\left\{\begin{array}{l}
y_{1}=\varphi_{1}\left(x_{1}, \ldots, x_{n}\right) \\
\vdots \\
y_{n}=\varphi_{n}\left(x_{1}, \ldots, x_{n}\right)
\end{array} \quad \text { and } \quad \psi=\left\{\begin{array}{l}
y_{1}=\psi_{1}\left(x_{1}, \ldots, x_{n}\right) \\
\vdots \\
y_{n}=\psi_{n}\left(x_{1}, \ldots, x_{n}\right)
\end{array} .\right.\right.
$$

Putting $f=y_{j}$ in $(1)$, we obtain

$$
\frac{\partial^{m}\left(\varphi_{j}-\psi_{j}\right)(a)}{\partial x^{\sigma}}=0
$$


for all $j=1, \ldots, n$ and for all multi-indexes $\sigma=\left(\sigma_{1}, \ldots, \sigma_{m}\right)$ such that $m \leqslant k$. Thus, the $k$-jet of $\varphi$ at $a$ can be identified with the vector

$$
[\varphi]_{a}^{k}=\left(\varphi_{1}(a), \ldots, \varphi_{n}(a), \frac{\partial \varphi_{1}}{\partial x_{1}}(a), \ldots \frac{\partial \varphi_{n}}{\partial x_{n}}(a), \ldots, \frac{\partial^{m} \varphi_{j}}{\partial x^{\sigma}}(a), \ldots\right),
$$

where $j=1, \ldots, n, \sigma=\left(\sigma_{1}, \ldots, \sigma_{m}\right), m \leqslant k$.

6.4. Fix points $a, b \in M$ and by $\mathbb{G}_{a, b}^{k}$ denote the set of all $k$-jets $[\varphi]_{a}^{k}$ of diffeomorphisms $\varphi$ such that $\varphi(a)=b$.

The operation of composition of diffeomorphisms gives the pairing

$$
\mathbb{G}_{a, b}^{k} \times \mathbb{G}_{b, c}^{k} \rightarrow \mathbb{G}_{a, c}^{k}
$$

taking a pair $\left([\varphi]_{a}^{k},[\varphi]_{b}^{k}\right)$ into $[\varphi \circ \psi]_{a}^{k}$.

In particular, if $a=b=c$, then this pairing turns the set $\mathbb{G}_{a, a}^{k}$ into a Lie group. For small numbers $k$ this group can be described easily.

So, if $k=0$, then the group $G_{a, a}^{0}=\left\{[\mathrm{id}]_{a}^{0}\right\}$ is trivial. If $k=1$, then the definition of a 1-jet $[\varphi]_{a}^{1}$ actually repeats the definition of a differential. Therefore, $[\varphi]_{a}^{1}$ can be identified with the differential

$$
\varphi_{*, a}: \mathrm{T}_{a} M \rightarrow \mathrm{T}_{a} M
$$

It is easy to check that each nonsingular linear transformation of the tangent space $\mathrm{T}_{a} M$ is a differential of some local diffeomorphism at the point $a$. Thus, the group $\mathbb{G}_{a, a}^{1}$ coincides with the general linear group $\mathrm{GL}\left(\mathrm{T}_{a} M\right)$.

6.5. In order to describe the group $\mathbb{G}_{a, a}^{k}$ for $k \geqslant 2$, consider the successive epimorphisms of groups

$$
\pi_{k, k-1}: \mathbb{G}_{a, a}^{k} \rightarrow \mathbb{G}_{a, a}^{k-1}
$$

and their kernels $\mathbb{H}_{a}^{k}$.

Proposition. For $k \geqslant 2$, the group $\mathbb{H}_{a}^{k}$ is a connected commutative Lie group. Moreover, $\mathbb{H}_{a}^{k}$ is isomorphic to the tensor product

$$
S^{k} \mathrm{~T}_{a}^{*} \otimes \mathrm{T}_{a}
$$

where $\mathrm{T}_{a}=\mathrm{T}_{a} M$ and $S^{k} \mathrm{~T}_{a}^{*}$ is the $k$-th symmetric power of the cotangent space $\mathrm{T}_{a}^{*}$. Proof. Let us first show that the group $\mathbb{H}_{a}^{k}$ is commutative. The condition that a $k$-jet $[\varphi]_{a}^{k}$ belongs to $\mathbb{H}_{a}^{k}$ means that

$$
[\varphi]_{a}^{k-1}=[\mathrm{id}]_{a}^{k-1}
$$

i.e. for every smooth function $f \in C^{\infty}(M)$

$$
\left(1-\varphi^{*}\right)(f) \in \mu_{a}^{k} .
$$


If in addition

$$
\left(1-\varphi^{*}\right)(f) \subset \mu_{a}^{k+1}
$$

then $[\varphi]_{a}^{k}$ is the identity element of $\mathbb{H}_{a}^{k}$.

Let $[\psi]_{a}^{k} \in \mathbb{H}_{a}^{k}$ and $k \geqslant 2$. Show that

$$
[\varphi \circ \psi]_{a}^{k}=[\psi \circ \varphi]_{a}^{k}
$$

Note that the following relations are true

$$
\begin{aligned}
& \left(\varphi^{*} \circ \psi^{*}\right)(f)=\left[\left(1-\varphi^{*}\right) \circ\left(1-\psi^{*}\right)-\left(1-\varphi^{*}\right)-\left(1-\psi^{*}\right)+1\right](f), \\
& \left(\psi^{*} \circ \varphi^{*}\right)(f)=\left[\left(1-\psi^{*}\right) \circ\left(1-\varphi^{*}\right)-\left(1-\varphi^{*}\right)-\left(1-\psi^{*}\right)+1\right](f) .
\end{aligned}
$$

Therefore

$$
\left(\varphi^{*} \circ \phi^{*}-\psi^{*} \circ \varphi^{*}\right)(f)=\left(1-\varphi^{*}\right) \circ\left(1-\psi^{*}\right)(f)-\left(1-\psi^{*} \circ\left(1-\varphi^{*}\right)(f) .\right.
$$

In addition,

$$
\left(1-\varphi^{*}\right)(f \cdot g)=f\left(1-\varphi^{*}\right)(g)+g\left(1-\psi^{*}\right)(f)-\left(f-\varphi^{*}(f)\right)\left(g-\varphi^{*}(g)\right)
$$

for all functions $f, g \in C^{\infty}(M)$.

From (4) it follows that if $\varphi$ satisfies condition (2), then

$$
\left(1-\varphi^{*}\right)\left(\mu_{a}^{l}\right) \subset \mu_{a}^{k+l-1}
$$

for all $l \geqslant 1$.

Since $[\psi]_{a}^{k} \in \mathbb{H}_{a}^{k}$, we have

$$
\left(1-\psi^{*}\right) \circ\left(1-\varphi^{*}\right)(f) \in\left(1-\psi^{*}\right)\left(\mu_{a}^{k}\right) \subset \mu_{a}^{2 k-1} .
$$

Since $2 k-1 \geqslant k+1$ for $k \geqslant 2$, from $(3)$ it follows that elements $[\varphi]_{a}^{k}$ and $[\psi]_{a}^{k}$ commute.

Further, relation (4) shows that the mapping

$$
X_{\varphi}: C^{\infty}(M) \rightarrow S^{k} \mathrm{~T}_{a}^{*}, \quad f \mapsto\left(1-\varphi^{*}\right)(f) \bmod \mu_{a}^{k+1}
$$

is a derivation and therefore can be represented as

$$
X_{\varphi}=\theta_{\varphi} \circ d_{a}
$$

where $\theta_{\varphi}: \mathrm{T}_{a}^{*} \rightarrow S^{k} \mathrm{~T}_{a}^{*}$ is a linear mapping and $d_{a}: C^{\infty}(M) \rightarrow \mathrm{T}_{a}^{*}$ is the differential at a point $a$. Thus, the mapping $[\varphi]_{a}^{k} \mapsto \theta_{\varphi}$ is a monomorphism of the groups $\mathbb{H}_{a}^{k}$ and $\operatorname{Hom}\left(\mathrm{T}_{a}^{*}, S^{k} \mathrm{~T}_{a}^{*}\right)$.

Now show that $[\varphi]_{a}^{k} \mapsto \theta_{\varphi}$ is an epimorphism. Indeed, every linear mapping $\theta$ : $\mathrm{T}_{a}^{*} \rightarrow S^{k} \mathrm{~T}_{a}^{*}$ can be identified with an element of the space $S^{k} \mathrm{~T}_{a}^{*} \otimes \mathrm{T}_{a}$ and therefore with a $k$-jet of some vector field $V$ on $M$. By $\left\{A_{t}\right\}$ denote the one-parameter group of translations along $V$. Then

$$
A_{t}^{*}(f)-f=t V(f) \bmod \mu_{a}^{k+1} .
$$

Thus, if we put $\varphi=A_{1}$, then $\theta=\theta_{\varphi}$.

6.6. Definition. The group $\mathbb{G}_{a, a}^{k}$ is called the full differential group of order $k$ (at the point $a \in M)$. 
Theorem. For $k \geqslant 2$, the full differential groups $\mathbb{G}_{a, a}^{k}$ are successive extensions of the group $\mathbb{G}_{a, a}^{1}=\mathrm{GL}\left(\mathrm{T}_{a} M\right)$ by commutative groups $\mathbb{H}_{a}^{k}=S^{k} \mathrm{~T}_{a}^{*} \otimes \mathrm{T}_{a}$ :

$$
\begin{gathered}
0 \rightarrow S^{2} \mathrm{~T}_{a}^{*} \otimes \mathrm{T}_{a} \rightarrow \mathbb{G}_{a, a}^{2} \stackrel{\pi_{2,1}}{\longrightarrow} \mathrm{GL}\left(\mathrm{T}_{a}\right) \rightarrow 1 \\
0 \rightarrow S^{3} \mathrm{~T}_{a}^{*} \otimes \mathrm{T}_{a} \rightarrow \mathbb{G}_{a, a}^{3} \stackrel{\pi_{3,2}}{\longrightarrow} \mathbb{G}_{a, a}^{2} \rightarrow 1 \\
\vdots \\
0 \rightarrow S^{k} \mathrm{~T}_{a}^{*} \otimes \mathrm{T}_{a} \rightarrow \mathbb{G}_{a, a}^{k} \stackrel{\pi_{k, k-1}}{\longrightarrow} \mathbb{G}_{a, a}^{k-1} \rightarrow 1 .
\end{gathered}
$$

6.7. As an illustration, let us describe coordinate representation of multiplication in $\mathbb{G}_{a, a}^{2}$.

Let $x_{1}, \ldots, x_{n}$ be local coordinates in a neighborhood of a point $a \in M$ and $x_{1}(a)=$ $\cdots=x_{n}(a)=0$. Then every 2 -jet $[\varphi]_{a}^{2}$ determined by a diffeomorphism $\varphi, \varphi(a)=a$, can be given as

$$
[\varphi]_{a}^{2}=\left(\left\|\frac{\partial \varphi_{j}}{\partial x_{i}}(0)\right\|,\left\|\frac{\partial^{2} \varphi_{j}}{\partial x_{l} \partial x_{s}}(0)\right\|\right)
$$

Then the following relation is true for $i=1, \ldots, n$ :

$$
\varphi^{*}\left(x_{i}\right)=\sum_{j} \frac{\partial \varphi_{i}}{\partial x_{j}}(0) x_{j}+\frac{1}{2} \sum_{l, s} \frac{\partial^{2} \varphi_{i}}{\partial x_{l} \partial x_{s}}(0) x_{l} x_{s} \bmod \mu_{a}^{3} .
$$

Therefore, for the composition $[\varphi]_{a}^{2} \circ[\phi]_{a}^{2}$ we have

$$
\begin{gathered}
x_{i} \stackrel{[\psi]_{a}^{2}}{\mapsto} \sum_{j} \frac{\partial \psi_{i}}{\partial x_{j}}(0) x_{j}+\frac{1}{2} \sum_{l, s} \frac{\partial^{2} \psi_{i}}{\partial x_{l} \partial x_{s}}(0) x_{l} x_{s} \bmod \mu_{a}^{3} \stackrel{[\varphi]_{a}^{2}}{\mapsto} \\
\sum_{l, j} \frac{\partial \psi_{i}}{\partial x_{j}}(0) \frac{\partial \varphi_{j}}{\partial x_{l}}(0) x_{l}+\frac{1}{2} \sum_{j, l, s} \frac{\partial \psi_{i}}{\partial x_{j}}(0) \frac{\partial^{2} \varphi_{j}}{\partial x_{l} \partial x_{s}}(0) x_{l} x_{s}+ \\
\frac{1}{2} \sum_{l, s, r, t} \frac{\partial \psi_{i}}{\partial x_{l} \partial x_{s}}(0) \frac{\partial \varphi_{l}}{\partial x_{r}}(0) \frac{\partial \varphi_{s}}{\partial x_{t}}(0) x_{r} x_{t} \bmod \mu_{a}^{3} .
\end{gathered}
$$

It follows that

$$
\begin{aligned}
{[\varphi]_{a}^{2} \circ[\psi]_{a}^{2}=\left(\| \frac{\partial \psi_{i}}{\partial x_{j}}(0) \frac{\partial \varphi_{j}}{\partial x_{l}}(0)\right.} & \| \\
& \left.\left\|\frac{\partial \psi_{i}}{\partial x_{j}}(0) \frac{\partial \varphi_{j}}{\partial x_{l} \partial x_{s}}(0)+\frac{\partial^{2} \psi_{i}}{\partial x_{l} \partial x_{s}}(0) \frac{\partial \varphi_{l}}{\partial x_{r}}(0) \frac{\partial \varphi_{s}}{\partial x_{t}}(0)\right\|\right)
\end{aligned}
$$

Note that if $[\varphi]_{a}^{2}$ and $[\psi]_{a}^{2}$ belong to $\mathbb{H}_{a}^{2}$, then

$$
[\varphi]_{a}^{2}=\left(\left\|\delta_{i j}\right\|,\left\|\frac{\partial^{2} \varphi_{i}}{\partial x_{l} \partial x_{s}}(0)\right\|\right), \quad[\phi]_{a}^{2}=\left(\left\|\delta_{i j}\right\|,\left\|\frac{\partial^{2} \psi_{i}}{\partial x_{l} \partial x_{s}}(0)\right\|\right),
$$


and

$$
[\varphi]_{a}^{2} \circ[\psi]_{a}^{2}=\left(\left\|\delta_{i j}\right\|,\left\|\frac{\partial^{2} \varphi_{i}}{\partial x_{l} \partial x_{s}}(0)+\frac{\partial^{2} \psi_{i}}{\partial x_{l} \partial x_{s}}(0)\right\|\right),
$$

which is in accordance with proposition 6.5. (Here $\delta_{i j}$ is Kronecker delta.)

Remark. The group $\mathbb{G}_{a, a}^{2}$ is the semidirect product of the group $\mathbb{G}_{a, a}^{1} \cong \mathrm{GL}\left(\mathrm{T}_{a}\right)$ and the commutative ideal $\mathbb{H}_{a}^{2} \cong \mathrm{T}_{a} \otimes S^{2} \mathrm{~T}_{a}^{*}$ with the natural action of $\mathbb{G}_{a, a}^{1}$ on $\mathbb{H}_{a}^{2}$. However, for $k \geqslant 2, \mathbb{G}_{a, a}^{k+1}$ cannot be represented as the semidirect product of the groups $\mathbb{G}_{a, a}^{k}$ and $\mathbb{H}_{a}^{k+1}$ (i.e. the corresponding exact sequence is not splitting).

\section{$\S 7$. Affine structures}

7.1. Let us define an action of the full differential group $\mathbb{G}_{a, a}^{k}$ on the space of $k$-jets $N_{a}^{k}$. For $[L]_{a}^{k} \in N_{a}^{k}$ and $[\varphi]_{a}^{k} \in \mathbb{G}_{a, a}^{k}$, put

$$
[\varphi]_{a}^{k}\left([L]_{a}^{k}\right) \stackrel{\text { def }}{=}[\varphi(L)]_{a}^{k}
$$

Since the right-hand side of the equality is determined by the $k$-jet of the diffeomorphism $\varphi$ and the curve $L$ respectively, we see that the action is well-defined.

Proposition. The Lie group $\mathbb{H}_{a}^{k}$ acts transitively on fibres of the projection

$$
\pi_{k, k-1}: N_{a}^{k} \rightarrow N_{a}^{k-1}
$$

For $k \geqslant 2$, the stabilizer of an element $[L]_{a}^{k} \in N_{a}^{k}$ under this action is the subgroup

$$
\left(A n n \mathrm{~T}_{a} L\right) \circ S^{k-1} \mathrm{~T}_{a}^{*} \otimes \mathrm{T}_{a}+S^{k} \mathrm{~T}_{a}^{*} \otimes \mathrm{T}_{a} L,
$$

where Ann $\mathrm{T}_{a} L \subset \mathrm{T}_{a}^{*}$ is the annihilator of the straight line $\mathrm{T}_{a} L$ and $\circ$ is the sign of symmetric product.

Proof. It is clear that the action of $\mathbb{H}_{a}^{k}$ is transitive. Indeed, let $L$ and $L^{\prime}$ be curves such that their $(k-1)$-jets at a point $a \in L \cap L^{\prime}$ coincide. Then there exists a diffeomorphism $\varphi$ such that $\varphi$ coincides with the identity diffeomorphism up to the $(k-1)$-th order and the curves $L$ and $\varphi\left(L^{\prime}\right)$ coincide in some neighborhood of the point $a$. Therefore, $[\varphi]_{a}^{k} \in \mathbb{H}_{a}^{k}$ and $[\varphi]_{a}^{k}\left(\left[L^{\prime}\right]_{a}^{k}\right)=[L]_{a}^{k}$.

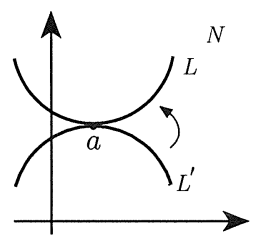

Fig. 15 
Let us now describe the stabilizer of an element $[L]_{a}^{k}$ under the action considered. Let $[\varphi]_{a}^{k}$ be an element of the stabilizer. Then the curves $L$ and $\varphi(L)$ have contact of order $\geqslant k+1$ at the point $a$. Therefore, for every function $f \in C^{\infty}(N)$ vanishing on $L$, the function $\left.\varphi^{*}(f)\right|_{L}$ has zero of order $\geqslant k+1$ at $a$. In terms of the operator $X_{\varphi}$ (see $6.5)$, this is equivalent to the fact that the restriction of the symmetric tensor $X_{\varphi}(f)$ to the straight line $\mathrm{T}_{a} L$ equals zero. Thus, $X_{\varphi}$ maps Ann $\mathrm{T}_{a} L$ into $\left(\mathrm{Ann} \mathrm{T}_{a} L\right) \circ S^{k-1} \mathrm{~T}_{a}^{*}$ and therefore

$$
X_{\varphi} \in\left(\operatorname{Ann} \mathrm{T}_{a} L\right) \circ S^{k-1} \mathrm{~T}_{a}^{*} \otimes \mathrm{T}_{a}+S^{k} \mathrm{~T}_{a} L .
$$

Corollary 1. Let $k \geqslant 2$ and let $[L]_{a}^{k}$ be an element of $N_{a}^{k}$. The fibre of the projection $\pi_{k, k-1}: N_{a}^{k} \rightarrow N_{a}^{k-1}$ is the affine space associated with the vector space

$$
S^{k} \mathrm{~T}_{a}^{*} L \otimes V_{a}
$$

where $V_{a}=\mathrm{T}_{a} N / \mathrm{T}_{a} L$ is the space normal to the curve $L$ at the point $a$.

Proof. The commutative group $\mathbb{H}_{a}^{k}$ acts transitively on the fibre $\pi_{k, k-1}^{-1}\left([L]_{a}^{k-1}\right)$. The stabilizer of this action has been calculated earlier. Thus, the abelian group

$$
S^{k} \mathrm{~T}_{a}^{*} \otimes \mathrm{T}_{a} /\left(\mathrm{Ann} \mathrm{T}_{a} L \circ S^{k-1} \mathrm{~T}_{a}^{*} \otimes \mathrm{T}_{a}+S^{k} \mathrm{~T}_{a} \otimes \mathrm{T}_{a} L\right) \simeq S^{k} \mathrm{~T}_{a}^{*} \otimes V_{a}
$$

acts transitively and effectively on the fibre $\pi_{k, k-1}^{-1}\left([L]_{a}^{k-1}\right)$.

Corollary 2. The fibre of the projection $\pi_{k, k-1}: J_{a}^{k}(\mathbb{R}, M) \rightarrow J_{a}^{k-1}(\mathbb{R}, M)$ is the affine space associated with the tangent space $\mathrm{T}_{a} M$.

Proof. It is sufficient to indicate the following isomorphisms:

$$
V_{a} \simeq \mathrm{T}_{a} M, \quad \mathrm{~T}_{a}^{*} L \simeq \mathbb{R} .
$$

Remark. Actually, $J_{a}^{k}(\mathbb{R}, M)$ is a vector space. As a base point, we can take the $k$-jet of the singular curve $\mathbb{R} \ni t \mapsto a \in M$.

7.2. Definition. The prolongation of order $k$ of a diffeomorphism $\varphi: N \rightarrow N$ is the diffeomorphism $\varphi^{(k)}: N^{k} \rightarrow N^{k}$ defined by

$$
\varphi^{(k)}\left([L]_{a}^{k}\right)=[\varphi(L)]_{a}^{k} .
$$

The basic properties of prolongations are as follows:

(i) $\varphi^{(k)} \circ \psi^{(k)}=(\varphi \circ \psi)^{(k)}$;

(ii) $(\text { id })^{(k)}=\mathrm{id}$;

(iii) for $k>s$ the diagram

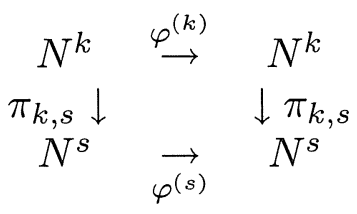


is commutative.

These properties can be easily verified.

7.3. For the space of $k$-jets of parametrized curves consider diffeomorphisms of the manifold $\mathbb{R} \times M$ that preserve the structure of the direct product and parametrization. More exactly, let $\varphi: \mathbb{R} \times M \rightarrow \mathbb{R} \times M$ be a diffeomorphism such that $\varphi$ is in agreement with the projection $\mathbb{R} \times M \rightarrow \mathbb{R}$ and induces the identical mapping on the base $\mathbb{R} ;$ in other words, $\varphi$ is a diffeomorphism such that the diagram

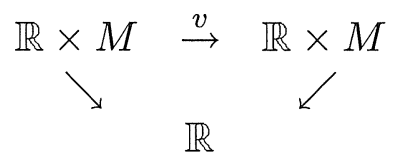

is commutative. Then $\varphi$ is called a gauge transformation.

It can be easily seen that every gauge transformation $\varphi$ can be written as

$$
\varphi:(t, a) \mapsto\left(t, \varphi_{t}(a)\right)
$$

where $\left\{\varphi_{t}\right\}$ is a one-parameter family of diffeomorphisms of $M$.

Gauge transformations act naturally on parametrized curves in $M$. If $\varphi$ is a gauge transformation and $x: \mathbb{R} \supset \mathcal{O} \rightarrow M$ is a parametrized curve, then by $\varphi(x)$ denote the curve $t \mapsto \varphi_{t}(x(t))$.

Definition. The prolongation of order $k$ of a gauge transformation $\varphi$ is a diffeomorphism $\varphi^{(k)}: J^{k}(\mathbb{R}, M) \rightarrow J^{k}(\mathbb{R}, M)$ defined by

$$
\varphi^{(k)}\left([x]_{t_{0}}^{k}\right)=[\varphi(x)]_{t_{0}}^{k}
$$

Prolongations $\varphi^{(k)}$ are restrictions of prolongations defined in the previous item to the open subset $J^{k}(\mathbb{R}, M) \subset(\mathbb{R} \times M)^{k}$. Therefore, they satisfy properties $(i)$, $(i i)$, and (iii) from 7.2.

7.4. Let $\varphi: N \rightarrow N$ be an arbitrary diffeomorphism and $\psi: N \rightarrow N$ a diffeomorphism such that $[\psi]_{a}^{k} \in \mathbb{H}_{a}^{k}$. Then $\left[\varphi \circ \psi \circ \varphi^{-1}\right]_{\varphi(a)}^{k} \in \mathbb{H}_{\varphi(a)}^{k}$ and therefore, for $k \geqslant 2$, the diffeomorphism $\varphi^{(k)}$ induces an affine transformation of fibres of the fibration $\pi_{k, k-1}$.

Summarizing all observations of this section, we obtain the following result:

\section{Theorem.}

(1) Let $k \geqslant 2$. Then the fibration $\pi_{k, k-1}: N^{k} \rightarrow N^{k-1}$ is an affine fibration and prolongations $\varphi^{(k)}: N^{k} \rightarrow N^{k}$ of diffeomorphisms of $N$ are affine automorphisms of fibres of this fibration. If $k=1$, then fibres of the fibration $\pi_{1,0}: N^{1} \rightarrow N$ are projectivisations of spaces tangent to $N$, while prolongations $\varphi^{(1)}: N^{1} \rightarrow N^{1}$ are projective transformations generated by the differential $\varphi_{*}: \mathrm{T} N \rightarrow \mathrm{T} N$. 
(2) For $k \geqslant 1$, the fibration $\pi_{k, k-1}: J^{k}(\mathbb{R}, M) \rightarrow J^{k-1}(\mathbb{R}, M)$ is a fibration of a vector bundle, and prolongations $\varphi^{(k)}$ of gauge transformations are linear automorphisms of this fibration.

7.5. As a larger group of transformations of the space $J^{k}(\mathbb{R}, M)$, consider diffeomorphisms of $\mathbb{R} \times M$ preserving fibres of the projection $\pi: \mathbb{R} \times M \rightarrow \mathbb{R}$. Every diffeomorphism $\varphi$ of this kind determines a parametrization (i.e. diffeomorphism) $\lambda: \mathbb{R} \rightarrow \mathbb{R}$ such that the diagram

$$
\begin{array}{ccc}
\mathbb{R} \times M & \stackrel{v}{\rightarrow} & \mathbb{R} \times M \\
\pi \downarrow & & \downarrow \pi \\
\mathbb{R} & \rightarrow & \mathbb{R}
\end{array}
$$

is commutative. Thus, every $\varphi$ is determined by a one-parameter family of diffeomorphisms $\varphi_{t}: M \rightarrow M, t \in \mathbb{R}$, and a parameterization $\lambda: \mathbb{R} \rightarrow \mathbb{R}:$

$$
\varphi:(t, a) \mapsto\left(\lambda(t), \varphi_{t}(a)\right) .
$$

In this case the prolongation $\varphi^{(k)}$ of $\varphi$ to the space $J^{k}(\mathbb{R}, M)$ is defined by

$$
\varphi^{(k)}\left([x(t)]_{t_{0}}^{k}\right)=\left[\varphi_{t}\left(x\left(\lambda^{-1}(t)\right)\right)\right]_{\lambda\left(t_{0}\right)}^{k} .
$$

If $k=1$, then $\varphi^{(1)}$ is determined by the differential $\varphi_{*}$ :

$$
\varphi^{(1)}\left([x(t)]_{t_{0}}^{1}\right)=\left(\lambda^{\prime}\left(t_{0}\right)\right)^{-1} \varphi_{*, a}\left(\dot{x}\left(t_{0}\right)\right)
$$

where $a=x\left(t_{0}\right)$.

The results of items 7.3 and 7.4 are also true for these, more general, transformations $\varphi$ and their prolongations.

\section{§8. Cartan distributions}

\subsection{Definition.}

(1) Let $L$ be a curve in $N$. The $k$-jet $j_{k}(L) \subset N^{k}$ of the curve $L$ is the curve

$$
j_{k}(L)=\left\{[L]_{a}^{k} \mid \forall a \in L\right\} .
$$

(2) Let $x: \mathcal{O} \rightarrow M$ be a parametrized curve. The $k$-jet $j_{k}(x): \mathcal{O} \rightarrow J^{k}(\mathbb{R}, M)$ of the curve $x$ is the curve in the space $J^{k}(\mathbb{R}, M)$ defined by

$$
j_{k}(x): t \mapsto[x(t)]_{t}^{k} .
$$

8.2. In this section we shall describe the basic structure on the space of $k$-jets of curves, namely, the Cartan distribution, which will allow to distinguish $k$-jets of curves among all curves in the space of $k$-jets.

Fix a point $A \in N^{k}$ and suppose $A=\left[L_{0}\right]_{a}^{k}$ for some curve $L_{0} \subset N$. Consider various curves of the form $j_{k}(L)$ passing through $A$, i.e. curves $j_{k}(L)$ such that $[L]_{a}^{k}=$ $\left[L_{0}\right]_{a}^{k}$. By $C_{A}$ denote the linear closure of the set of all tangent lines $\mathrm{T}_{A}\left(j_{k}(L)\right)$ to curves like this. 
Definition. The space $C_{A} \subset \mathrm{T}_{A}\left(N^{k}\right)$ is called the Cartan space. The distribution $C: A \mapsto C_{A}$ on the manifold $N^{k}$ is called the Cartan distribution.

We can similarly define Cartan spaces and distribution for the manifold $J^{k}(\mathbb{R}, M)$ of $k$-jets of parametrized curves.

8.3. The Cartan distribution can also be described by means of special differential 1 -forms.

Definition. A differential 1-form $\omega$ on $J^{k}(\mathbb{R}, M)$ is called horizontal if $\omega$ vanishes at vectors tangent to all fibres of the projection $\pi_{k}: J^{k}(\mathbb{R}, M) \rightarrow \mathbb{R}$.

By $\Lambda_{0}^{1}\left(J^{k}(\mathbb{R}, M)\right)$ denote the set of all horizontal 1-forms on $J^{k}(\mathbb{R}, M)$. It is obvious that this set is closed under addition and multiplication by smooth functions $f \in$ $C^{\infty}\left(J^{k}(\mathbb{R}, M)\right)$.

Note that if $\omega$ is a horizontal 1-form on $J^{k}(\mathbb{R}, M)$ such that for all parametrized curves $x$ in $M$

$$
j_{k}(x)^{*}(\omega)=0,
$$

then $\omega=0$. Thus, horizontal 1-forms are completely determined by their restrictions to $k$-jets of parametrized curves.

8.4. To every smooth function $f \in C^{\infty}\left(J^{k-1}(\mathbb{R}, M)\right)$ we assign a horizontal 1-form $\hat{d} f \in \Lambda_{0}^{1}\left(J^{k}(\mathbb{R}, M)\right)$ by means of the following property:

$$
\left(j_{k}(x)\right)^{*}(\hat{d} f)=d\left(j_{k-1}(x)^{*}(f)\right)
$$

for all parameterized curves $x: \mathbb{R} \rightarrow M$.

Proposition. The 1-form $\hat{d} f$ is well-determined by relation (2).

Proof. The fact that $\hat{d} f$ is unique follows from the property mentioned at the end of item 8.3. It remains to prove that $\hat{d} f$ exists. It is sufficient to note that every nonvertical vector $v \in \mathrm{T}_{A}\left(J^{k}(\mathbb{R}, M)\right)$, i.e. a vector such that $\left(\pi_{k}\right)_{*}(v) \neq 0$, can be made into a vector tangent to a curve of the form $j_{k}(x)$, by adding some vertical (with respect to the projection $\pi_{k, k-1}$ ) vector. But the forms standing in both sides of (2) vanish at vectors tangent to all fibres of the projection $\pi_{k, k-1}$. Therefore, relation (2) enables us to calculate the value of $\hat{d} f$ at $v$ and thereby determines the 1 -form $\hat{d} f$.

8.5. Theorem. The mapping $f \mapsto \hat{d} f$ determines the operator

$$
\hat{d}: C^{\infty}\left(J^{k-1}(\mathbb{R}, M)\right) \rightarrow \Lambda_{0}^{1}\left(J^{k}(\mathbb{R}, M)\right)
$$

satisfying the following properties:

1) $\hat{d}$ is linear over $\mathbb{R}$;

2) $\hat{d}$ is a derivation:

$$
\hat{d}(f g)=\pi_{k, k-1}^{*}(f) \hat{d} g+\pi_{k, k-1}^{*}(g) \hat{d} f .
$$


Proof. We shall prove only the second property, since the proof of the first one is absolutely similar. Let us restrict both sides of equality (3) to an arbitrary curve $j_{k}(x)$ :

$$
\begin{gathered}
\left(j_{k}(x)\right)^{*}(\hat{d}(f g))=d j_{k-1}^{*}(f g)=j_{k-1}^{*}(f) d j_{k-1}^{*}(g)+j_{k-1}^{*}(g) d j_{k-1}^{*}(f) \\
\left(j_{k}(x)\right)^{*}\left(\pi_{k, k-1}(f) \hat{d} g+\pi_{k, k-1}(g) \hat{d} f\right)= \\
=j_{k-1}^{*}(f) j_{k}(x)^{*}(\hat{d} g)+j_{k-1}(g) j_{k}(x)^{*}(\hat{d} f)= \\
=j_{k-1}^{*}(f) d j_{k-1}^{*}(g)+j_{k-1}^{*}(g) d j_{k-1}^{*}(f)
\end{gathered}
$$

We see that the restrictions coincide. Since the forms in both sides of (3) are horizontal, from the property mentioned in 8.3 it follows that the forms coincide.

8.6. Consider the action of the operator $\hat{d}$ in local coordinates. Let

$$
f=f\left(t, u^{1}, \ldots, u^{n}, p_{1}^{1}, \ldots, p_{1}^{n} ; \ldots ; p_{k}^{1}, \ldots, p_{k}^{n}\right)
$$

be a smooth function defined on $J^{k-1}(\mathbb{R}, M)$. (For the sake of convenience in the sequel we shall write it as $f=f\left(t, u^{i}, p_{l}^{j}\right)$.) From theorem 8.5 it follows that

$$
\hat{d} f=\frac{\partial f}{\partial t} \hat{d} t+\sum_{i} \frac{\partial f}{\partial u^{i}} \hat{d} u^{i}+\sum_{i, j} \frac{\partial f}{\partial p_{j}^{i}} \hat{d} p_{j}^{i} .
$$

Thus, in order to calculate $\hat{d} f$, it is sufficient to calculate the 1 -forms $\hat{d} t, \hat{d} u^{i}, \hat{d} p_{l}^{j}$. Since all these forms are horizontal, they can be written in the form $g\left(t, u^{i}, p_{l}^{j}\right) d t$, where $l \leqslant k$. Let us begin with $\hat{d} t$ and let

$$
\hat{d t}=A\left(t, u^{i}, p_{l}^{j}\right) d t
$$

Then from (2) it follows that

$$
A\left(t, x^{i}(t),\left(x^{j}\right)^{(l)}(t)\right) d t=d t
$$

Therefore, $A \equiv 1$ and

$$
\hat{d} t=d t
$$

Further, if

$$
\hat{d} p_{s}^{r}=B\left(t, u^{i}, p_{l}^{j}\right) d t
$$

then from (2) it follows that

$$
B\left(t, x^{i}(t),\left(x^{j}\right)^{(l)}(t)\right) d t=d\left(x^{r}(t)\right)^{(s)}=\left(x^{r}(t)\right)^{(s+1)} d t .
$$


Therefore, $B=p_{s+1}^{r}$ and

$$
\hat{d} p_{s}^{r}=p_{s+1}^{r} d t
$$

Similarly we obtain $\hat{d} u^{i}=p_{1}^{i} d t$. Finally,

$$
\hat{d} f=D(f) d t
$$

where

$$
D=\frac{\partial}{\partial t}+\sum_{i} p_{1}^{i} \frac{\partial}{\partial u^{i}}+\sum_{i, j \leqslant k-1} p_{j+1}^{i} \frac{\partial}{\partial p_{j}^{i}}
$$

is the operator of total differentiation.

8.7. Definition. Let $f \in C^{\infty}\left(J^{k-1}(\mathbb{R}, M)\right)$. The following differential 1-form on $J^{k}(\mathbb{R}, M)$ is called the Cartan form determined by $f$ :

$$
\omega(f)=d \pi_{k, k-1}^{*}(f)-\hat{d} f
$$

Proposition. Cartan forms vanish on curves of the form $j_{k}(x)$

Proof. Since $\pi_{k, k-1} \circ j_{k}(x)=j_{k-1}(x)$, we have

$$
\left(j_{k}(x)\right)^{*}\left(d\left(\pi_{k, k-1}^{*} f\right)\right)=d\left(j_{k}(x)^{*}\left(\pi_{k, k-1}^{*} f\right)\right)=d\left(j_{k-1}(x)^{*} f\right)
$$

Now, using (2), we obtain

$$
\begin{gathered}
\left(j_{k}(x)\right)^{*}(\omega(f))=\left(j_{k}(x)\right)^{*}\left(d \pi_{k, k-1}^{*} f\right)-\left(j_{k}(x)\right)^{*} \hat{d} f= \\
=d\left(j_{k-1}(x)^{*} f\right)-d\left(j_{k-1}(x)^{*} f\right)=0 .
\end{gathered}
$$

8.8. Using representation (5) for the operator $\hat{d}$, we see that Cartan forms can be written in local coordinates as

$$
\omega(f)=\sum_{i} \frac{\partial f}{\partial u^{i}} \omega\left(u^{i}\right)+\sum_{i, j \leqslant k-1} \frac{\partial f}{\partial p_{j}^{i}} \omega\left(p_{j}^{i}\right),
$$

where

$$
\begin{gathered}
\omega\left(u^{1}\right)=d u^{1}-p_{1}^{1} d t, \ldots, \omega\left(u^{n}\right)=d u^{n}-p_{1}^{n} d t \\
\omega\left(p_{j}^{i}\right)=d p_{j}^{i}-p_{j+1}^{i} d t .
\end{gathered}
$$

This calculation immediately leads to the following result: 


\section{Theorem.}

(1) The Cartan distribution on the manifold $J^{k}(\mathbb{R}, M)$ can be considered as the annihilator of Cartan forms $\omega(f)$ for all $f \in C^{\infty}\left(J^{k-1}(\mathbb{R}, M)\right)$.

(2) Let $q$ be a curve in $J^{k}(\mathbb{R}, M)$ that can be parametrized by the function $\pi_{k}$ : $J^{k}(\mathbb{R}, M) \rightarrow \mathbb{R}$. Then $q$ is a $k$-jet of a parameterized curve in $M$ if and only if all Cartan forms vanish on $q$.

8.9. In conclusion, we shall give one more description of the Cartan distribution. For this purpose note that every $A=[x]_{t_{0}}^{k} \in J^{k}(\mathbb{R}, M)$ determines the straight line $l(A)$ tangent to the curve $j_{k-1}(x)$ at the point $A^{\prime}=\pi_{k, k-1}(A)$. The sum of $l(A)$ and the space $F_{A^{\prime}}$ tangent to the fibre $\pi_{k-1}^{-1}\left(t_{0}\right)$ at the point $A^{\prime}$ gives the whole space $\mathrm{T}_{A^{\prime}}\left(J^{k-1}(\mathbb{R}, M)\right)$ (see Fig. 16).

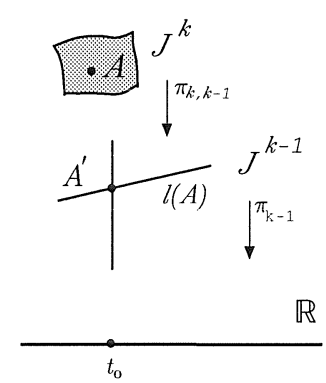

Fig. 16

Consider the operator

$$
U_{A}: \mathrm{T}_{A}\left(J^{k}(\mathbb{R}, M)\right) \rightarrow F_{A^{\prime}}
$$

that takes a vector $v \in \mathrm{T}_{A}\left(J^{k}(\mathbb{R}, M)\right)$ into the projection of $\left(\pi_{k, k-1}\right)_{*}(v)$ to $F_{A^{\prime}}$ along $l(A)$.

Theorem. $\operatorname{ker} U_{A}=C_{A}$.

Proof. Note that if $\operatorname{dim} M=n$, then $\operatorname{dim} C_{A}=n+1$ and $\operatorname{dim} \operatorname{ker} U_{A}=n+1$. Therefore, it is sufficient to prove that $C_{A} \subset \operatorname{ker} U_{A}$. But the operator $U_{A}$ vanishes on vectors tangent to curves of the form $j_{k}(x)$. Thus, $C_{A}=\operatorname{ker} U_{A}$.

8.10. As an illustration consider the simplest case, that is $k=1$. In this case the fibre of the projection $\pi_{0}: M \times \mathbb{R} \rightarrow M$ coincides with $M$. Therefore, $F_{A^{\prime}}=\mathrm{T}_{a} M$, where $a=\pi_{0}\left(A^{\prime}\right)=\pi_{1}(A)$, and the operator $U_{A}$ has the form:

$$
U_{A}: \mathrm{T}_{A}(\mathrm{~T} M \times \mathbb{R}) \rightarrow \mathrm{T}_{a} M .
$$

Identifying $\mathrm{T}_{a} M$ with the subspace of $\mathrm{T}_{A}(\mathrm{~T} M \times \mathbb{R})$ tangent to the fibre of the projection $\mathrm{T} M \times \mathbb{R} \rightarrow M \times \mathbb{R}$, we obtain the operator field

$$
A \mapsto U_{A}: \mathrm{T}_{A}(\mathrm{~T} M \times \mathbb{R}) \rightarrow \mathrm{T}_{A}(\mathrm{~T} M \times \mathbb{R})
$$


on the manifold of 1 -jets.

It is immediate from the definition of $U_{A}$ that

$$
U_{A}^{2}=0
$$

for all points $A \in J^{1}(\mathbb{R}, M)$.

Let us describe the operator field $U$ in local coordinates. Suppose $t, u^{1}, \ldots, u^{n}$, $p_{1}^{1}, \ldots, p_{1}^{n}$ are coordinates in some neighborhood of a point $A \in J^{1}(\mathbb{R}, M)$. By theorem 8.9 the operator $U_{A}$ vanishes on $C_{A}$. Hence

$$
U_{A}=\sum_{i=1}^{n} v_{i} \otimes w\left(u^{i}\right),
$$

where vectors $v_{1}, \ldots, v_{n}$ are linearly independent. By definition

$$
U_{A}\left(\frac{\partial}{\partial u_{i}}\right)=\frac{\partial}{\partial p_{1}^{i}},
$$

so that

$$
U_{A}=\sum_{i=1}^{n} \frac{\partial}{\partial p_{1}^{i}} \otimes w\left(u^{i}\right)
$$

or

$$
U_{A}=\sum_{i=1}^{n} \frac{\partial}{\partial p_{1}^{i}} \otimes\left(d u^{i}-p_{1}^{i} d t\right) .
$$

Since $J^{1}(\mathbb{R}, M)=\mathbb{R} \times \mathrm{T} M$, we see that the operator field $U$ can be written as

$$
U=-v \otimes d t+U_{0}
$$

where $v$ is a vector field on the tangent bundle T $M$ and $U_{0}$ is on operator field on T $M$.

The vector field $v$ is called Liouville field. It can be described invariantly as the vertical vector field on $\mathrm{T} M$ such that the one-parameter group $A_{t}$ of translations along this field has the form:

$$
A_{t}:(u, p) \mapsto\left(u, e^{t} p\right) .
$$

The operator field $U_{0}$ is called the tangent structure. These operators are also nilpotent:

$$
U_{0, B}^{2}=0 \quad \text { for all } B \in \mathrm{T} M .
$$

In contrast to the operator field $U$, kernel

$$
\operatorname{Ker} U_{0, B}=\operatorname{Im} U_{0, B}
$$

coincides with the space tangent to the fibre of the projection T $M \rightarrow M$ for all points $B \in \mathrm{T} M$. 


\section{§9.DifFERENTIAL EQUATIONS}

In this section we shall once more revert to basic concepts of differential equations and give their invariant interpretation. We shall consider only spaces of jets of parametrized curves, since in the majority of cases extension of the basic concepts to the case of spaces $N^{k}$ can be made automatically.

\subsection{Definition.}

(1) A system of ordinary differential equations of order $k$ on a manifold $M$ is a smooth submanifold $\mathcal{E} \subset J^{k}(\mathbb{R}, M)$ of codimension equal to $\operatorname{dim} M$.

(2) A solution (or more exactly, a classical solution) of this system is a parametrized curve $x: \mathcal{O} \rightarrow M$ such that the image of $\mathcal{O}$ by the $k$-jet $j_{k}(x): \mathcal{O} \rightarrow J^{k}(\mathbb{R}, M)$ lies in $\mathcal{E}$.

We shall say that the system $\mathcal{E}$ is solvable with respect to the derivative of the highest order if the mapping

$$
\pi_{k, k-1}: \mathcal{E} \rightarrow J^{k-1}(\mathbb{R}, M)
$$

is a diffeomorphism.

If $\mathcal{E}$ is solvable with respect to the derivative of the highest order, then to every point $A \in J^{k-1}(\mathbb{R}, M)$ we can assign its inverse image $\hat{A} \in \mathcal{E}$ by $\pi_{k, k-1}$ and therefore the straight line $l(A)=l(\hat{A}) \subset C(A)$ (see item 8.9). Let $X_{A} \in C(A)$ be a vector on $l(A)$ such that the projection of $X_{A}$ on $\mathbb{R}$ is the vector field $\frac{\partial}{\partial t}$. Then the system $\mathcal{E}$ can be identified with the vector field $X$ on $J^{k-1}(\mathbb{R}, M)$ lying in the Cartan distribution. Thus, the following result is true:

9.2 Proposition. There is a one-to-one correspondence between systems of differential equations of order $k$ solvable with respect to the derivative of the highest order and vector fields on $J^{k-1}(\mathbb{R}, M)$ lying in the Cartan distribution.

9.3. Let $k=2$. A system of differential equations of order 2 solvable with respect to the derivative of the second order can be identified with a vector field $X$ on the manifold $J^{1}(\mathbb{R}, M)=\mathrm{T} M \times \mathbb{R}$. This field can be written as

$$
X=\frac{\partial}{\partial t}+Y_{t}
$$

where $\left\{Y_{t}\right\}$ is a one-parameter family of vector fields on the manifold T $M$. Since $X$ lies in the Cartan distribution, we have

$$
U(X)=U_{0}(X)-v=0
$$

where $v$ is the Liouville vector field on $\mathrm{T} M$.

Thus, we can identify (see (1)) a system of differential equations of order 2 solvable with respect to the derivative of the second order with a one-parameter family of vector fields $Y_{t}$ on the tangent bundle $\mathrm{T} M$, where $\left\{Y_{t}\right\}$ satisfies the additional condition

$$
U_{0}\left(Y_{t}\right)=v
$$


9.4. The operator field $U$ on $J^{1}(\mathbb{R}, M)$ gives additional derivations of the algebra of exterior differential forms. Consider the inner derivation

$$
i_{U}: \Lambda^{j}\left(J^{1}(\mathbb{R}, M)\right) \rightarrow \Lambda^{j}\left(J^{1}(\mathbb{R}, M)\right)
$$

where

$$
\left(i_{U} w\right)\left(X_{1}, \ldots, X_{j}\right)=w\left(U\left(X_{1}\right), \ldots, X_{j}\right)+\cdots+w\left(X_{1}, \ldots, U\left(X_{j}\right)\right)
$$

for all differential forms $w$ and vector fields $X_{1}, \ldots, X_{j}$ on $J^{1}(\mathbb{R}, M)$.

If $j=0$ and $f \in \Lambda^{0}\left(J^{1}(\mathbb{R}, M)\right)=C^{\infty}\left(J^{1}(\mathbb{R}, M)\right)$, then by definition put

$$
i_{U}(f)=0
$$

It can be easily verified that the following result is true:

Proposition. The inner derivation $i_{U}$ is a 0 -degree derivation of the exterior algebra; in other words,

(i) $i_{U}$ is linear over $C^{\infty}\left(J^{1}(\mathbb{R}, M)\right)$;

(ii) $i_{U}\left(w_{1} \wedge w_{2}\right)=i_{U} w_{1} \wedge w_{2}+w_{1} \wedge i_{U} w_{2}$ for all differential forms $w_{1}, w_{2}$ on $J^{1}(\mathbb{R}, M)$.

Let $d$ be the standard exterior derivation. By $d_{U}$ denote the commutator of $d$ and the inner derivation $i_{U}$ :

$$
d_{U}=d \circ i_{U}-i_{U} \circ d: \Lambda^{j}\left(J^{1}(\mathbb{R}, M)\right) \rightarrow \Lambda^{j+1}\left(J^{1}(\mathbb{R}, M)\right)
$$

Using the previous proposition, we obtain the following result:

Theorem. The operator $d_{U}$ is a 1-degree derivation of the exterior algebra; in other words,

(i) $d_{U}$ is $\mathbb{R}$-linear;

(ii) $d_{U}\left(w_{1} \wedge w_{2}\right)=d_{U} w_{1} \wedge w_{2}+(-1)^{j} w_{1} \wedge d_{U} w_{2}$ if $w_{1} \in \Lambda^{j}\left(J^{1}(\mathbb{R}, M)\right)$.

In addition, $d \circ d_{U}+d_{U} \circ d=0$ and for an arbitrary function $f$ the following relation holds:

$$
d_{U}^{2}(f)+d_{U}(f) \wedge d t=0
$$

9.5. Let us now describe the action of $d_{U}$ in local coordinates. First, note that

$$
i_{U}(d t)=i_{U}\left(d u^{i}\right)=0 \quad \text { and } \quad i_{U}\left(d p_{1}^{j}\right)=w^{j} .
$$

Therefore, for an arbitrary function $f$ we have

$$
d_{U}(f)=i_{U}(d f)=\frac{\partial f}{\partial t} i_{U}(d t)+\sum_{j}\left(\frac{\partial f}{\partial u^{j}} i_{U}\left(d u^{j}\right)+\frac{\partial f}{\partial p_{1}^{j}} i_{U}\left(d p_{1}^{j}\right)\right)
$$


or

$$
d_{U}(f)=\sum_{j} \frac{\partial f}{\partial p_{1}^{j}} w_{j} .
$$

Further, since $d \circ d_{U}+d_{U} \circ d=0$, we obtain

$$
d_{U}(d t)=-d\left(d_{U}(t)\right)=0, d_{U}\left(d u^{j}\right)=-d\left(d_{U} u^{j}\right)=0, d_{U}\left(d p_{i}^{j}\right)=-d w_{j} .
$$

Relations (4) and (5) enable us to calculate the value of the operator $d_{U}$ at an arbitrary differential form.

9.6. In practice, most of differential equations of the second order appear as EulerLagrange equations of a variational problem. In this item we shall give a brief description of how to obtain equations of this sort. Every function $L$ defined on $J^{1}(\mathbb{R}, M)$ can be regarded as a Lagrangian of a variational problem.

Consider the functional

$$
I: x(t) \mapsto \int_{a}^{b}\left(j_{1}(x)\right)^{*}(L) d t
$$

defined on the space of smooth curves in $M$. The problem will remain unchanged if we add any linear combination of Cartan forms to the differential 1-form $L d t$ under the integral sign.

The differential 1-form

$$
\theta_{L}=d_{U} L+L d t
$$

is called the structure form of the variational problem. The Lagrangian $L$ is called non-degenerate if the form $\theta_{L}$ determines a contact structure in $J^{1}(\mathbb{R}, M)$.

Consider the kernel of the differential 2 -form $d \theta_{L}$ defined on the odd-dimensional manifold $J^{1}(\mathbb{R}, M)$ :

$$
\left.\operatorname{Ker} d \theta_{L}=\left\{X \in \mathrm{T}\left(J^{1}(\mathbb{R}, M)\right) \mid X\right\lrcorner d \theta_{L}=0\right\} .
$$

Its dimension is equal or greater than 1. If the Lagrangian $L$ is non-degenerate, then $\operatorname{dim} \operatorname{Ker} d \theta_{L}=1$.

By $Z_{L}$ denote the vector field on $J^{1}(\mathbb{R}, M)$ such that $Z_{L}$ forms a basis of $\operatorname{Ker} d \theta_{L}$ and its projection on $\mathbb{R}$ is $\frac{\partial}{\partial t}$.

Thus, the vector field $Z_{L}$ is uniquely defined by the following conditions:

$$
\begin{aligned}
& \text { i) } \left.Z_{L}\right\lrcorner \theta_{L}=1 ; \\
& \text { ii) } \left.Z_{L}\right\lrcorner d \theta_{L}=0 .
\end{aligned}
$$

The fundamental theorem of variational calculus can be reformulated as follows:

Theorem. Extremals of non-degenerate variational problem (6) coincide with trajectories of the vector field $Z_{L}$. The vector field $Z_{L}$ determines a system of differential equations of order 2 on the manifold $M$, that is a system of Euler-Lagrange equations. 
CHAPTER II

\section{SYMMETRIES}

The problem of solution of differential equations or, what is the same, of finding integral manifolds of distributions is a problem of transcendental complexity. The idea of symmetry turns out to be very constructive in solution of this problem, as well as in solution of any mathematical problem though. Such is the case in the study of algebraic equations, where solubility (say, by radicals) of an algebraic equation is determined by its Galois group or (what is the same) its symmetry group. A similar situation takes place in the study of differential equations. However, in the latter case it is possible to linearize the problem, i.e. to pass to Lie algebras of symmetries. We are now going on to the fundamental concept of this book.

\section{§1. Symmetries OF Distributions}

1.1. Let $E$ be a distribution on a manifold $M$. First, we give a preliminary definition of a symmetry.

Definition. A symmetry of the distribution $E$ is a diffeomorphism $\varphi: M \rightarrow M$ such that

$$
\varphi_{*}(E(a))=E(\varphi(a))
$$

for all elements $a \in M$.

By $\operatorname{sym} E$ denote the set of all symmetries of $E$. Let us point out some obvious properties of symmetries.

i) The set $\operatorname{sym} E$ is a group with respect to composition of diffeomorphisms.

ii) Let $L \subset M$ be an integral manifold of the distribution $E$ and $\varphi$ a symmetry of $E$. Then the manifold $\varphi(L) \subset M$ is also integral. Moreover, if $L$ is a maximal integral manifold, then so is $\varphi(L)$.

Thus, symmetries enable us to construct new integral manifolds, starting from already known ones.

Obviously, symmetries just defined have local nature. So, we can consider local symmetries of a distribution, defining them as local diffeomorphisms $\varphi: \mathcal{O} \rightarrow \mathcal{O}^{\prime}$ such that $\varphi$ satisfies condition (1) for all $a \in \mathcal{O}$. All local symmetries of a distribution form a pseudogroup.

1.2. Assume that $E$ is the distribution given by differential 1 -forms $w_{1}, . ., w_{m}$ :

$$
E=\mathcal{F}\left(w_{1}, \ldots, w_{m}\right)
$$

In addition suppose that the covectors $w_{1, a}, \ldots, w_{m, a}$ are linearly independent at all points $a \in M$ (or at all points of some domain, if local symmetries are involved). 
It is clear that condition (1) is equivalent to the following condition:

$$
\mathcal{F}\left(w_{1}, \ldots, w_{m}\right)=\mathcal{F}\left(\varphi^{*}\left(w_{1}\right), \ldots, \varphi^{*}\left(w_{m}\right)\right) .
$$

Therefore, there exists a smooth matrix-valued function $A: a \mapsto\left\|A_{i j}(a)\right\|, i, j=$ $1, \ldots, m$, such that for all points $a$

$$
\operatorname{det}\left\|A_{i j}(a)\right\| \neq 0
$$

and

$$
\begin{aligned}
& \varphi^{*}\left(w_{1}\right)=A_{11} w_{1}+\cdots+A_{1 m} w_{m}, \\
& \vdots \\
& \varphi^{*}\left(w_{m}\right)=A_{m 1} w_{1}+\cdots+A_{m m} w_{m} .
\end{aligned}
$$

Relations (3) are very convenient when we use local coordinates. Let $q_{1}, \ldots, q_{n}$ be local coordinates in a neighborhood of a point $a \in M$ and $q_{1}^{\prime}, \ldots, q_{n}^{\prime}$ local coordinates in a neighborhood of $\varphi(a)$, where $\varphi: M \rightarrow M$ is a diffeomorphism.

Then in these coordinates $\varphi$ has the form:

$$
\begin{aligned}
& q_{1}^{\prime}=Q_{1}\left(q_{1}, \ldots, q_{n}\right), \\
& \vdots \\
& q_{n}^{\prime}=Q_{n}\left(q_{1}, \ldots, q_{n}\right) .
\end{aligned}
$$

Suppose that the forms $w_{1}, \ldots, w_{n}$ have the form

$$
\left\{\begin{array}{l}
w_{1}=w_{11}(q) d q_{1}+\cdots+w_{1 n}(q) d q_{n} \\
\vdots \\
w_{m}=w_{m 1}(q) d q_{n}+\cdots+w_{m n}(q) d q_{n}
\end{array}\right.
$$

in a neighborhood of $a$ and

$$
\left\{\begin{array}{l}
w_{1}=w_{11}^{\prime}(q) d q_{1}^{\prime}+\cdots+w_{1 n}^{\prime}(q) d q_{n}^{\prime} \\
\vdots \\
w_{m}=w_{m 1}^{\prime}(q) d q_{n}^{\prime}+\cdots+w_{m n}^{\prime}(q) d q_{n}^{\prime}
\end{array}\right.
$$

in a neighborhood of $\varphi(a)$. Then relations (3) take the form

$$
\sum_{j} w_{i j}^{\prime} \frac{\partial Q_{j}}{\partial q_{k}}=\sum_{j} A_{i j} w_{j k} .
$$

It can be written in matrix form as

$$
\Omega^{\prime} \frac{\partial Q}{\partial q}=A \Omega
$$


where $\Omega$ and $\Omega^{\prime}$ are the following matrices:

$$
\Omega=\left\|w_{i j}\right\|, \Omega^{\prime}=\left\|w_{i j}^{\prime}\right\| .
$$

In oder to avoid indeterminacy connected with the function $A$, we rewrite relations (3) in the equivalent form:

$$
\left\{\begin{array}{l}
\varphi^{*}\left(w_{1}\right) \wedge w_{1} \wedge \cdots \wedge w_{m}=0 \\
\vdots \\
\varphi^{*}\left(w_{m}\right) \wedge w_{1} \wedge \cdots \wedge w_{m}=0
\end{array}\right.
$$

Then relation $(7)$ means that all $(m+1)$-th order minors of the matrices

$$
B_{j}=\left\|\begin{array}{c}
\sum_{j} w_{j i}^{\prime} \frac{\partial Q_{j}}{\partial q_{1}}, w_{11}, \ldots, w_{m 1} \\
\vdots \\
\sum_{j} w_{j i}^{\prime} \frac{\partial Q_{j}}{\partial q_{n}}, w_{1 n}, \ldots, w_{m n}
\end{array}\right\|, j=1, \ldots, m,
$$

are equal to 0.

\subsection{Examples.}

1. Let $E$ be the distribution on $M=\mathbb{R}^{2}$ given by the 1 -form $w=d q_{1}$ (see fig.17). Symmetries of this distribution are transformation of the plane preserving the family of vertical straight lines.

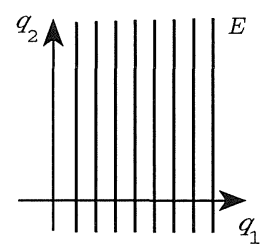

Fig. 17

In this case relations (7) have the form:

$$
\frac{\partial Q_{1}}{\partial q_{1}}=A, \frac{\partial Q_{1}}{\partial q_{2}}=0
$$

Thus, symmetries of the distribution $E$ are transformations of the form:

$$
\varphi:\left(q_{1}, q_{2}\right) \mapsto\left(\left(Q_{1}\left(q_{1}\right), Q_{2}\left(q_{1}, q_{2}\right)\right) .\right.
$$

2. Consider the Cartan distribution $C$ on the manifold $J^{1}=J^{1}(\mathbb{R}, \mathbb{R})$, i.e. the distribution given by the differential 1-form

$$
w=d u-p d t .
$$


Symmetries of this distribution are called contact transformations. They have the form

$$
\varphi:(t, u, p) \mapsto(T((t, u, p), U(t, u, p), P(t, u, p))
$$

where

$$
\left\{\begin{array}{l}
\frac{\partial U}{\partial u}-P \frac{\partial T}{\partial u}=A \\
\frac{\partial U}{\partial t}-P \frac{\partial T}{\partial t}=-A p \\
\frac{\partial U}{\partial p}-P \frac{\partial T}{\partial p}=0 .
\end{array}\right.
$$

Substituting $A$ from the first equation into second one, we obtain

$$
\left\{\begin{array}{l}
\frac{d U}{d t}-P \frac{d T}{d t}=0 \\
\frac{\partial U}{\partial p}-P \frac{\partial T}{\partial p}=0
\end{array}\right.
$$

where

$$
\frac{d}{d t}=\frac{\partial}{\partial t}+P \frac{\partial}{\partial u}
$$

is the operator of total differentiation with respect to $t$. In its turn, system (10) may be rearranged to give a single relation on the functions $U$ and $T$

$$
\frac{d U}{d t} \frac{\partial T}{\partial p}-\frac{\partial U}{\partial p} \frac{d T}{d t}=0
$$

assuming that

$$
P=\frac{\partial U}{\partial p} / \frac{\partial T}{\partial p}
$$

3. Legendre transformations are contact transformations of $J^{1}(\mathbb{R}, \mathbb{R})$ of the form:

$$
\varphi:(t, u, p) \mapsto(-p, u-p t, t)
$$

Here $T=-p, U=u-p t, P=t$ and obviously, conditions (11) and (12) are satisfied.

1.4. Relations (7) can be considered as a system of nonlinear differential equations on the functions $Q_{1}, \ldots, Q_{n}$. In this respect it appears that the procedure of finding symmetries of a distribution is hardly simpler than that of finding its integral manifolds. However, a system like this has an advantage over an arbitrary nonlinear system: it is a Lie system of equations, i.e. a system of nonlinear differential equations such that its solutions form a group. The problem of finding symmetries can be linearized and simplified if we pass to infinitesimal symmetries.

Definition. A vector field $X$ on a manifold $M$ is called an infinitesimal symmetry of a distribution $E$ if the local one-parameter group $\varphi_{t}$ of translations along $X$ consists of local symmetries of the distribution $E$.

In the sequel we shall mainly use infinitesimal symmetries. For brevity sake, we shall simply call them symmetries. 
By $\operatorname{sym}(E)$ denote the set of all infinitesimal symmetries of a distribution $E$. By $\mathcal{D}(E)$ denote the set of all vector fields lying in a distribution $E$ :

$$
\mathcal{D}(E)=\left\{X \mid X_{a} \in E_{a} \forall a \in M\right\} .
$$

If $E=\mathcal{F}\left(w_{1}, \ldots, w_{n}\right)$, then

$$
\mathcal{D}(E)=\left\{X \mid w_{1}(X)=\cdots=w_{m}(X)=0\right\} .
$$

Theorem. The following conditions are equivalent:

(1) $X \in \operatorname{sym}(E)$;

(2) $L_{X}\left(w_{i}\right)=\sum_{j=1}^{m} c_{i j} w_{j}, i=1, \ldots, m$, where $E=\mathcal{F}\left(w_{1}, \ldots, w_{m}\right)$ and $c_{i j}$ are smooth functions defined on $M$;

(3) $L_{X}\left(w_{i}\right) \wedge w_{1} \wedge \cdots \wedge w_{m}=0, i=1, \ldots, m$;

(4) if $Y \in \mathcal{D}(E)$ then $[X, Y] \in \mathcal{D}(E)$.

Here by $L_{X}$ we denote the Lie derivative with respect to a vector field $X$.

Proof.

$1 \Rightarrow 2$. Let $X \in \operatorname{sym}(E)$ and let $\varphi_{t}$ be the one-parameter group of translations along the vector field $X$. From relation (3) it follows that

$$
\varphi_{t}^{*}\left(w_{i}\right)=\sum_{j=1}^{m} A_{i j}(t) w_{j}
$$

Let us differentiate this equality with respect to $t$ and then put $t=0$. This leads us to $(2)$, where

$$
c_{i j}=\left.\frac{\partial A_{i j}}{\partial t}\right|_{t=0}
$$

$2 \Leftrightarrow 3$. Both of the conditions mean that covectors $L_{X}\left(w_{i}\right)$ are linear combinations of the covectors $w_{1}, \ldots, w_{m}$.

$3 \Rightarrow 1$. Consider the differential $(m+1)$-forms

$$
\Omega_{i}(t)=\varphi_{t}^{*}\left(w_{i}\right) \wedge w_{1} \cdots \wedge w_{m}, i=1, \ldots, m .
$$

Let us show that $\Omega_{i}(t)=0$. For this purpose note that

$$
\frac{\partial \Omega_{i}(t)}{\partial t}=\varphi_{t}^{*}\left(L_{X}\left(w_{i}\right)\right) \wedge w_{1} \cdots \wedge w_{m}=\sum_{j=1}^{m} \varphi_{t}^{*}\left(c_{i j}\right) \Omega_{j}(t) .
$$

Thus the vector-valued function

$$
\Omega(t)=\left(\Omega_{1}(t), \ldots, \Omega_{m}(t)\right)
$$

is a solution of a homogeneous system of linear differential equations. Since $\Omega(0)=0$, we see that $\Omega(t)=0$.

$2 \Leftrightarrow 4$. By $i_{Z}$ denote the operator of inner multiplication by a vector field $Z$. Since $i_{Y}\left(w_{j}\right)=0$ and

we obtain

$$
i_{[X, Y]}=\left[L_{X}, i_{Y}\right]
$$

$$
w_{j}([X, Y])=i_{[X, Y]}\left(w_{j}\right)=\left[L_{X}, i_{Y}\right]\left(w_{j}\right)=-i_{Y}\left(L_{X}\left(w_{j}\right)\right)
$$


Corollary 1. The set $\operatorname{sym}(E)$ is a Lie algebra over $\mathbb{R}$ with respect to commutation of vector fields.

Corollary 2. A distribution $E$ is completely integrable if and only if

$$
\mathcal{D}(E) \subset \operatorname{sym}(E) \text {. }
$$

1.6. Examples. Let us describe symmetry algebras $\operatorname{sym} E$ for distributions considered in 1.3

1. Here $w=d q_{1}$. For a vector field $X$ of the form

$$
X=a\left(q_{1}, q_{2}\right) \frac{\partial}{\partial q_{1}}+b\left(q_{1}, q_{2}\right) \frac{\partial}{\partial q_{2}}
$$

condition (3) of theorem 1.5. has the form:

$$
L_{X}(w) \wedge w=d a \wedge d q_{1}=\frac{\partial a}{\partial q_{2}} d q_{1} \wedge d q_{2}=0 .
$$

Therefore,

$$
\operatorname{sym} E=\left\{a \frac{\partial}{\partial q_{1}}+b \frac{\partial}{\partial q_{2}} \mid \frac{\partial a}{\partial q_{2}}=0\right\} .
$$

2. Let $w=d u-p d t$. Infinitesimal symmetries of the Cartan distribution $C=\mathcal{F}(w)$ are also called contact vector fields. If $X \in \operatorname{sym}(C)$ has the form

$$
X=a \frac{\partial}{\partial u}+b \frac{\partial}{\partial p}+c \frac{\partial}{\partial t},
$$

then

$$
\begin{aligned}
& L_{X}(w) \wedge w=(d a-b d t-p d c) \wedge(d u-p d t)= \\
& \begin{aligned}
\left(-\frac{\partial a}{\partial p}+p \frac{\partial c}{\partial p}\right) d u \wedge d p+\left(-\frac{\partial a}{\partial t}-p \frac{\partial a}{\partial u}+b+p \frac{\partial c}{\partial t}+p^{2} \frac{\partial c}{\partial u}\right) d u \wedge d t+ \\
\quad\left(-p \frac{\partial a}{\partial p}+p^{2} \frac{\partial c}{\partial p}\right) d p \wedge d t=0 .
\end{aligned}
\end{aligned}
$$

Therefore, $X \in \operatorname{sym} C$ if and only if

$$
\left\{\begin{array}{l}
\frac{\partial a}{\partial p}-p \frac{\partial c}{\partial p}=0 \\
\frac{\partial a}{\partial t}-p \frac{\partial c}{\partial t}+p\left(\frac{\partial a}{\partial u}-p \frac{\partial c}{\partial u}\right)-b=0
\end{array}\right.
$$

Put

$$
f=a-p c .
$$


Then from relations (13) it follows that

$$
c=-\frac{\partial f}{\partial p}, b=\frac{\partial f}{\partial t}, \text { and } a=f-p \frac{\partial f}{\partial p} .
$$

Finally,

$$
\operatorname{sym}(C)=\left\{X=-\frac{\partial f}{\partial p} \frac{\partial}{\partial t}+\frac{\partial f}{\partial t} \frac{\partial}{\partial p}+\left(f-p \frac{\partial f}{\partial p}\right) \frac{\partial}{\partial u} \mid f \in C^{\infty}\left(J^{1}(\mathbb{R}, \mathbb{R})\right\} .\right.
$$

By $X_{f}$ denote the contact vector field corresponding to a function $f=f(t, u, p)$ :

$$
X_{f}=-\frac{\partial f}{\partial p} \frac{\partial}{\partial t}+\frac{d f}{d t} \frac{\partial}{\partial p}+\left(f-p \frac{\partial f}{\partial p}\right) \frac{\partial}{\partial u} .
$$

Thus, the set of contact vector fields is isomorphic to the space $C^{\infty}\left(J^{1}(\mathbb{R}, \mathbb{R})\right)$ of smooth functions on $J^{1}(\mathbb{R}, \mathbb{R})$.

Let us now describe a structure of a Lie algebra on $\operatorname{sym}(C)$ or, what is the same, on $C^{\infty}\left(J^{1}(\mathbb{R}, \mathbb{R})\right)$.

It is obvious that

$$
X_{\lambda_{1} f_{1}+\lambda_{2} f_{2}}=\lambda_{1} X_{f_{1}}+\lambda_{2} X_{f_{2}}
$$

for arbitrary functions $f_{1}, f_{2} \in C^{\infty}\left(J^{1}(\mathbb{R}, \mathbb{R})\right)$ and constants $\lambda_{1}, \lambda_{2} \in \mathbb{R}$, so that

$$
X=X_{f} \in \operatorname{sym}(C) \mapsto f \in C^{\infty}\left(J^{1}(\mathbb{R}, \mathbb{R})\right)
$$

is an isomorphism of vector spaces.

Define the Lagrange bracket $[f, g]$ of two functions $f$ and $g$ by

$$
\left[X_{f}, X_{g}\right]=X_{[f, g]} .
$$

From (15) it follows that the Lagrange bracket of functions $f$ and $g$ can be written as

$$
[f, g]=f \frac{\partial g}{\partial u}-g \frac{\partial f}{\partial u}+\frac{d f}{d t} \frac{\partial g}{\partial p}-\frac{d g}{d t} \frac{\partial f}{\partial p} .
$$

Relation (16) shows that $C^{\infty}\left(J^{1}(\mathbb{R}, \mathbb{R})\right)$ is a Lie algebra with respect to Lagrange bracket (17).

Summarizing all that has been said in this example, we obtain the following result:

Theorem. Let $C$ denote the Cartan distribution on $J^{1}(\mathbb{R}, \mathbb{R})$. Every contact vector field $X=X_{f} \in \operatorname{sym} C$ has form (15). The mapping $X_{f} \mapsto f$ establishes an isomorphism of the Lie algebra sym $C$ onto the Lie algebra $C^{\infty}\left(J^{1}(\mathbb{R}, \mathbb{R})\right)$, where $C^{\infty}\left(J^{1}(\mathbb{R}, \mathbb{R})\right)$ is considered as a Lie algebra with respect to Lagrange bracket (17).

1.7. Let us now revert to description of symmetries for general differential systems. Conditions of Frobenius' theorem, reformulated in terms of symmetries as

$$
\mathcal{D}(E) \subset \operatorname{sym}(E),
$$

show that a completely integrable distribution $E$ has rather many symmetries, which can be found with the use of only linear algebra.

Note that symmetries $X \in \mathcal{D}(E)$ let invariant each maximal integral manifold of the distribution $E$. This allows to construct integral manifolds by induction on dimension. 
Definition. Let $E$ be an arbitrary distribution and $X$ a vector field such that

$$
X \in \operatorname{sym}(E) \cap \mathcal{D}(E) .
$$

Then $X$ is called a characteristic vector field of the distribution $E$.

The property just mentioned still remains valid for characteristic vector fields of an arbitrary distribution:

Proposition. Let $X$ be a characteristic vector field of a distribution $E$ and $L \subset M$ a maximal integral manifold of $E$. Then $L$ is invariant under the action of $X$.

Proof. Assume that the converse is true. Suppose that the vector field $X$ is not tangent to $L$ and let $\varphi_{t}$ be the one-parameter group of translations along $X$. Then for sufficiently small $t \in \mathbb{R}$, the subset

$$
\hat{L}=\bigcup_{t} \varphi_{t}(L) \subset M
$$

is an integral manifold. Indeed, at each point $b \in \varphi_{t}(L)$ the tangent space $\mathrm{T}_{b}(\hat{L})$ is the direct sum of the subspace $\mathrm{T}_{b}\left(\varphi_{t}(L)\right)$ and the straight line $\mathbb{R} X_{b}$ (see Fig. 18).

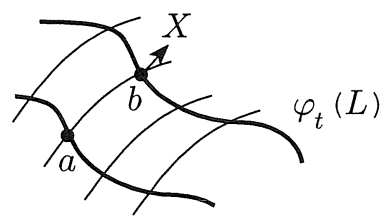

Fig. 18

But $\varphi_{t}$ is a symmetry and therefore

$$
\mathrm{T}_{b}\left(\varphi_{t}(L)\right)=\left(\varphi_{t}\right)_{*}\left(\mathrm{~T}_{a} L\right) \subset E(b) .
$$

Since $X$ is a characteristic vector field, we have $X_{b} \in E_{b}$ so that

$$
\mathrm{T}_{b}(\hat{L})=\mathrm{T}_{b}\left(\varphi_{t}(L)\right)+\mathbb{R} X_{b} \subset E(b) .
$$

On the other hand, for dimension reasons $\operatorname{dim} \hat{L}>\operatorname{dim} L$, which is impossible since $L$ is a maximal integral manifold.

1.8. Theorem. (Criterion of characteristicity.) Assume that a distribution $E$ is given by differential 1 -forms $w_{1}, \ldots, w_{m}$, i.e. $E=\mathcal{F}\left(w_{1}, \ldots, w_{m}\right)$. A vector field $X$ is characteristic for $E$ if and only if the following conditions hold:

$$
\begin{aligned}
& \text { (i) } w_{1}(X)=\cdots=w_{m}(X)=0 \quad \text { and } \\
& \text { (ii) } i_{X}\left(d w_{j}\right) \wedge w_{1} \wedge \cdots \wedge w_{m}=0, j=1, \ldots, m .
\end{aligned}
$$


Proof. Indeed, condition (i) means that $X \in \mathcal{D}(E)$ and therefore

$$
L_{X}\left(w_{j}\right)=i_{X}\left(d w_{j}\right)
$$

Then condition (ii) is equivalent to the condition that $X \in \operatorname{sym}(E)$.

1.9. Conditions (19) can be considered as a system of linear algebraic equations in coefficients of a vector field $X$. By $\operatorname{Char}_{a} E$ denote the solution space of this system at a point $a \in M$ :

$$
\left.\operatorname{Char}_{a} E=\left\{X_{a} \in E(a) \mid\left(X_{a}\right\lrcorner d w_{j}\right) \wedge w_{1} \wedge \cdots \wedge w_{m}=0, j=1, \ldots, m\right\} .
$$

Definition. The codimension of the space $\operatorname{Char}_{a} E$ is called the class of the distribution $E$ at the point $a$ and is denote by $\operatorname{cl}_{a}(E)$ :

$$
\operatorname{cl}_{a}(E)=\operatorname{dim} M-\operatorname{dim} \operatorname{Char}_{a} E .
$$

In the sequel we shall assume that the function

$$
\mathrm{cl}: a \mapsto \mathrm{cl}_{a}(E)
$$

is locally constant on $M$. Then the family of vector spaces $a \mapsto \operatorname{Char}_{a} E$ determines the distribution Char $E$ on $M$ called the characteristic distribution. Its codimension is called the class of the distribution $E$ and is denoted by $\operatorname{cl}(E)$.

Theorem (E.Cartan). The characteristic distribution is completely integrable.

Proof. Let $X$ and $Y$ be characteristic vector fields of the distribution $E$. It is sufficient to show that their commutator $[X, Y]$ is also a characteristic vector field belonging to $E$. Since $\operatorname{sym} E$ is a Lie algebra, we have $[X, Y] \in \operatorname{sym} E$. Moreover, since $L_{X}\left(w_{j}\right)$ is a linear combination of the forms $w_{1}, \ldots, w_{m}$, we obtain

$$
w_{j}([X, Y])=i_{[X, Y]}\left(w_{j}\right)=\left[L_{X}, i_{Y}\right]\left(w_{j}\right)=-i_{y}\left(L_{X}\left(w_{j}\right)\right)=0 .
$$

1.10. Remark. Proposition 1.7 combined with Cartan theorem shows that maximal integral manifolds of the characteristic distribution Char $E$ lie in those of the distribution $E$.

1.11. Examples. Let us describe the characteristic distributions for the distributions considered in examples 1.6.

1. Here $E=\mathcal{F}(w), w=d q_{1}$, and

$$
\operatorname{sym}(E)=\left\{X=a\left(q_{1}\right) \frac{\partial}{\partial q_{1}}+b\left(q_{1}, q_{2}\right) \frac{\partial}{\partial q_{2}}\right\} .
$$


Therefore,

$$
\operatorname{Char} E=\{X \in \operatorname{sym}(E) \mid w(X)=0\}=\left\{X=b\left(q_{1}, q_{2}\right) \frac{\partial}{\partial q_{2}}\right\} .
$$

Thus, Char $E=E$.

2. $C=\mathcal{F}(w)$, where $w=d u-p d t$, and $\operatorname{sym}(C)=\left\{X_{f}\right\}$.

If $X_{f} \in \mathcal{D}(C)$, then

$$
w\left(X_{f}\right)=f=0
$$

Thus, Char $E=0$.

1.12. Let $E=\mathcal{F}\left(w_{1}, \ldots, w_{m}\right)$. The class of the distribution $E$ has a simple interpretation: $r=\operatorname{cl}(E)$ is the least natural number such that the differential 1-forms $w_{1}, \ldots, w_{m}$ can be expressed in terms of $r$ variables $q_{1}, \ldots, q_{r}$ :

$$
w_{j}=\sum_{s=1}^{r} w_{j s}\left(q_{1}, \ldots, q_{r}\right) d q_{s}, \quad j=1, \ldots, r .
$$

In order to prove this statement, let us choose local coordinates $\left(q_{1}, \ldots, q_{n}\right)$ so that the vector fields

$$
X_{r+1}=\frac{\partial}{\partial q_{r+1}}, \ldots, X_{n}=\frac{\partial}{\partial q_{n}}
$$

from a basis of the distribution Char $E$. Suppose that the distribution $E$ is given by differential 1-forms of the form:

$$
w_{i}^{\prime}=\sum_{j=1}^{m} \lambda_{i j}(q) w_{j}
$$

where $i=1, \ldots, m,\left\|\lambda_{i j}\right\|$ is a non-singular matrix, and

$$
L_{X_{s}}\left(w_{i}^{\prime}\right)=0, s=r+1, \ldots, n .
$$

Since Char $E \subset E$, we see that in the local coordinates $\left(q_{1}, \ldots, q_{n}\right)$

$$
w_{i}^{\prime}=\sum_{j=1}^{r} w_{i j}(q) d q_{j}
$$

In addition,

$$
L_{X_{s}}\left(w_{i}^{\prime}\right)=\sum_{j=1}^{r} \frac{\partial w_{i j}}{\partial q_{s}} d q_{j}, s=r+1, \ldots, n .
$$

Therefore, equations (21) are solvable with respect to unknown functions $\lambda_{i j}$.

1.13. Proposition. Char $E$ is an ideal in the Lie algebra $\operatorname{sym} E$. 
Proof. Let $X \in$ Char $E$ and $Y \in \operatorname{sym} E$. Let us show that $[X, Y] \in$ Char $E$. Indeed, let $w$ be a differential 1-form annihilating the distribution $E$. Since $i_{X} w=0$ and the form $L_{Y} w$ also annihilates $E$, we have

$$
i_{[X, Y]} w=-\left[L_{Y}, i_{X}\right] w=i_{X} L_{Y} w=0
$$

1.14. By $\mathcal{M}_{E}$ denote the set of all maximal integral manifolds of a distribution $E$. Each symmetry $X \in \operatorname{sym} E$ generates a flow in $\mathcal{M}_{E}$ : if $\left\{\varphi_{t}\right\}$ is the one-parameter group of translations along the field $X$ and $L \in \mathcal{M}_{E}$, then $L_{t}=\varphi_{t}(L) \in \mathcal{M}_{E}$. In addition, if $X \in$ Char $E$ then $L_{t}=L$. Therefore, characteristic symmetries generate trivial flows in the solution space. With this in mind, elements of the quotient Lie algebra

$$
\operatorname{shuf} E=\operatorname{sym} E / \text { Char } E
$$

are called shuffling symmetries, and the Lie algebra shuf $E$ itself is called the algebra of shuffing symmetries.

1.15. Examples. For distributions considered in examples 1.11, we have

1. shuf $E \simeq\left\{a\left(q_{1}\right) \frac{\partial}{\partial q_{1}}\right\}$

2. $\operatorname{shuf} E=\operatorname{sym} E$.

1.16 Example. Let $\left(q_{1}, q_{2}\right)$ be coordinates on the plane $\mathbb{R}^{2}$. Consider the completely integrable distribution on $\mathbb{R}^{2}$ given by the differential 1 -form

$$
w=d q_{2}-F\left(q_{1}, q_{2}\right) d q_{1}
$$

In this case, the characteristic distribution is generated by the vector field

$$
\frac{\partial}{\partial q_{1}}+F\left(q_{1}, q_{2}\right) \frac{\partial}{\partial q_{2}}
$$

Therefore, shuffling symmetries can be identified with vector fields of the form:

$$
X=G\left(q_{1}, q_{2}\right) \frac{\partial}{\partial q_{2}} .
$$

The condition

$$
L_{X}(w) \wedge w=0
$$

is equivalent to the following differential equation with respect to the function $G$ :

$$
\frac{\partial G}{\partial q_{1}}+F \frac{\partial G}{\partial q_{2}}-G \frac{\partial F}{\partial q_{2}}=0
$$

Thus, the Lie algebra shuf $E$ can be identified with the solution space of equation (22). 


\section{$\S 2$. Contact Geometry}

In this section we shall consider the simplest distributions - distributions of codimension 1. Each distribution of this kind considered locally has the form $E=\mathcal{F}(w)$ for some differential 1-form $w$. The characteristic distribution Char $E$ can be described as

$$
\left.\operatorname{Char}_{a} E=\{X \in E(a) \mid(X\lrcorner d w) \wedge w=0\right\} .
$$

In other words, $\operatorname{Char}_{a} E \subset E_{a}$ is the kernel of the 2-form $\Omega_{a}=\left.d w\right|_{E_{a}}$. Therefore, the codimension of $\operatorname{Char}_{a} E$ in $E_{a}$ is an even number, whereas $\operatorname{cl}_{a}(E)$ is odd.

\subsection{Definition.}

(1) A distribution $E$ on a manifold $M$ is called a contact distribution if $\operatorname{cl}(E)=$ $\operatorname{dim} M$.

(2) A differential 1-form $w$ on a manifold $M$ is said to be contact if the distribution $\mathcal{F}(w)$ is contact.

(3) A manifold $M$ supplied with a contact distribution is called a contact manifold.

\subsection{Examples.}

(1) The Cartan distribution on $J^{1}(\mathbb{R}, \mathbb{R})=\mathbb{R}^{3}$ is contact. Moreover, the form

$$
w=d z-y_{1} d x_{1}-\cdots-y_{k} d x_{k}
$$

on $\mathbb{R}^{2 k+1}$ is contact.

(2) Let $N$ be an arbitrary 2-dimensional manifold. The Cartan distribution on the manifold $N^{1}$ is contact.

(3) A non-degenerate Lagrangian $L \in C^{\infty}\left(J^{1}(\mathbb{R}, M)\right)$ determines the contact form $\theta_{L}=d_{U} L+L d t$ (see 9.6, chapter 1$)$.

2.3. The condition for a 1-form $w$ to be contact can be reformulated as follows: a 1 -form $w$ is contact if for any point $a \in M$, the restriction of the 2 -form $d w$ to the hyperplane $E_{a}$ is non-singular. Therefore, a contact manifold $M$ is odd-dimensional: $\operatorname{dim} M=2 k+1$.

If $\operatorname{dim} M=2 k+1$, then the condition that $d w$ be non-singular on $E_{a}$ is equivalent to the condition that

$$
w \wedge(d w)^{k} \neq 0
$$

Thus, a manifold supplied with a contact form is orientable.

2.4. Definition. Symmetries of a contact distribution are called contact vector fields.

2.5. Let $E=\mathcal{F}(w)$ be a contact distribution and $X$ a contact vector field of $E$. Then

$$
L_{X}(w) \wedge w=0
$$

or

$$
\left.L_{X}(w)\right)=\lambda_{X} w
$$

for some smooth function $\lambda=\lambda_{X} \in C^{\infty}(M)$. 
Example. Let $M$ be an odd-dimensional manifold and $w$ a differential 1-form on $M$. The form $d w$ is singular on $M$. Therefore, there exists a nonzero vector field $Y$ such that

$$
Y\lrcorner d w=0 .
$$

If $w$ is a contact form, then this field is unique up to multiplication by a function. Let us normalize the field $Y$ to be equal to 1 at $w$. By $X_{1}$ denote the field obtained. Then

$$
\left.\left.X_{1}\right\lrcorner d w=0 \text { and } X_{1}\right\lrcorner w=1 \text {. }
$$

Since

$$
\left.\left.L_{X_{1}}(w)=X_{1}\right\lrcorner d w+d\left(X_{1}\right\lrcorner w\right)=0,
$$

we see that the field $X_{1}$ is contact.

2.6. The next theorem is a generalization of results obtained in example 1.3(2) of the previous section.

Theorem. Let $w$ be a contact form on a manifold $M$. Then each contact vector field $X$ on $M$ is uniquely determined by the function $f=w(X)$. In addition, for any function $f \in C^{\infty}(M)$ there exists a unique contact vector field $X_{f}$ such that the following conditions hold:

(i) $w\left(X_{f}\right)=f$,

(ii) $L_{X_{f}}(w)=X_{1}(f) w$,

(iii) $X_{f+g}=X_{f}+X_{g}$,

(iv) $X_{f g}=f X_{g}+g X_{f}-f g X_{1}$,

(v) $X_{f}(g)+X_{g}(f)=X_{1}(f) g+X_{1}(g) f$.

Here by $X_{1}$ we denote the contact vector field corresponding to the function $f=1$ and determined from relations (3).

Proof. Let $X$ be a contact vector field on $M$ satisfying condition (2). Let us write $X$ as

$$
X=f X_{1}+Y,
$$

where $Y$ is a field lying in the distribution $E=\mathcal{F}(w)$, i.e. $w(Y)=0$. Using

$$
\left.\left.w(X)=f \text { and } L_{X}(w)=X\right\lrcorner d w+d(X\lrcorner w\right),
$$

we obtain

$$
X\lrcorner d w=\lambda w-d f .
$$

But since $\left.f X_{1}\right\lrcorner d w=0$, we have

$$
Y\lrcorner d w=\lambda w-d f .
$$

The differential form in the right-hand side of (4) vanishes on $X_{1}$. Therefore,

$$
(\lambda w-d f)\left(X_{1}\right)=\lambda-X_{1}(f)=0,
$$


so that

$$
\lambda=X_{1}(f)
$$

Then relation (4) takes the form:

$$
Y\lrcorner d w=X_{1}(f) w-d f .
$$

In order to prove the first part of the theorem, it remains to note that the mapping $E(a) \ni Y \mapsto Y\lrcorner d w \in E^{*}(a)$ is an isomorphism and therefore (6) uniquely determines the vector field $Y$.

It is clear that relation (iii) is satisfied. So, we shall dwell on proving that (iv) and (v) are also valid. From (4) it follows that

$$
\left.X_{f}\right\lrcorner d w=X_{1}(f) w-d f .
$$

Consider the values of both sides of the equality at a vector field $X_{g}$. We obtain:

$$
d w\left(X_{f}, X_{g}\right)=X_{1}(f) g-X_{g}(f)
$$

By the same reasoning,

$$
d w\left(X_{g}, X_{f}\right)=X_{1}(g) f-X_{f}(g)
$$

Adding (7) and (8), we obtain (v).

By $Z$ denote the right-hand side of relation (iv). Then

$$
w(Z)=f g
$$

and

$\left.\left.Z\lrcorner d w=f X_{g}\right\lrcorner d w-g X_{f}\right\lrcorner d w=\left(f X_{1}(g)+g X_{1}(f)\right) w-f d g-g d f=X_{1}(f g) w-d(f g)$, which implies that $Z=X_{f g}$.

2.7. Definition. The function $f=w(X)$ is called the generating function of the contact vector field $X$.

2.8. Let $f$ and $g$ be functions defined on a contact manifold $M$. The Lagrange bracket $[f, g]$ of $f$ and $g$ is the generating function of the vector field $\left[X_{f}, X_{g}\right]$ :

$$
[f, g]=w\left(\left[X_{f}, X_{g}\right]\right)
$$


Theorem. Lagrange bracket turns $C^{\infty}(M)$ into a Lie algebra over the field $\mathbb{R}$ and

$$
[f, g]=X_{f}(g)-X_{1}(f) g .
$$

Proof. The fact that $C^{\infty}(M)$ is a Lie algebra with respect to Lagrange bracket is immediate from the definition. Let us check that formula (10) is valid. Indeed,

$$
i_{\left[X_{f}, X_{g}\right]} w=\left[L_{X_{f}}, i_{X_{g}}\right](w)=X_{f}(g)-X_{1}(f) g .
$$

2.9. Theorem.[J.Darboux]. Let $w$ and $w^{\prime}$ be contact forms on a manifold $M$. Then for any point $a \in M$ there exists a local diffeomorphism $\varphi$ such that

$$
\varphi(a)=a \text { and } \varphi^{*}\left(w^{\prime}\right)=w .
$$

Proof. Without loss of generality it can be assumed that

$$
w_{a}=w_{a}^{\prime} \text { and } d_{a} w=d_{a} w^{\prime}
$$

Consider the one-parameter family of 1-forms

$$
w_{t}=t w^{\prime}+(1-t) w .
$$

Then $w_{0}=w$ and $w_{1}=w^{\prime}$.

We shall be searching for a one-parameter family of local diffeomorphisms $\psi_{t}$ such that $\psi_{t}(a)=a, \psi_{0}=i d$, and

$$
\psi_{t}^{*}\left(w_{t}\right)=w .
$$

Differentiation of both sides of (11) gives

$$
\frac{d}{d t} \psi_{t}^{*}\left(w_{t}\right)=\psi_{t}^{*}\left(L_{X_{t}} w_{t}+\dot{w}_{t}\right)=0
$$

where $\dot{w}_{t}=d w_{t} / d t=w^{\prime}-w$ and $\left\{X_{t}\right\}$ is a family of vector fields defined on some neighborhood of $a$ such that translations along $X_{t}$ in the time from $t=0$ to $t$ coincide with $\psi_{t}$. From (12) it follows that

$$
\left.X_{t}\right\lrcorner d w_{t}+d\left(w_{t}\left(X_{t}\right)\right)=\dot{w} .
$$

As in the proof of theorem 2.6, let us write the vector field $X_{t}$ as

$$
X_{t}=f_{t} X_{1}^{t}+Y_{t}
$$

where $w_{t}\left(Y_{t}\right)=0$ and $X_{1}^{t}$ is the contact vector field such that $w_{t}\left(X_{1}^{t}\right)=1$. Then relation (13) can be rewritten as

$$
\left.Y_{t}\right\lrcorner d w_{t}=\dot{w}-d f_{t} .
$$

Let $\left\{f_{t}\right\}$ be a one-parameter family of smooth functions on $M$ such that in some neighborhood of the point $a$

$$
\left(\dot{w}-d f_{t}\right)\left(X_{1}^{t}\right)=0
$$

or

$$
X_{1}^{t}\left(f_{t}\right)=\dot{w}\left(X_{t}\right)
$$

Thus, choosing functions $f_{t}$ and the vector field $Y_{t}$ so that $f_{t}(a)=0, f_{t}$ satisfy (15), and $Y_{t}$ satisfies (14), we see that the vector fields $X_{t}$ vanish at the point $a$ and the family $\left\{\psi_{t}\right\}$ satisfies condition (11). Therefore, $\psi_{1}^{*}\left(w^{\prime}\right)=w$. 
Corollary. Let $M$ be a manifold of dimension $2 k+1, w$ a contact form on $M$, and $a \in M$. Then it is possible to choose local coordinates $\left(z, x_{1}, \ldots, x_{k}, y_{1}, \ldots, y_{k}\right)$ in a neighborhood of a such that $z(a)=x_{1}(a)=\cdots=x_{k}(a)=y_{1}(a)=\cdots=y_{k}(a)=0$ and

$$
w=d z-y_{1} d x-\cdots-y_{k} d x_{k} .
$$

Coordinate systems like this are called canonical.

2.10. System of linear equations (6) can be easily solved in canonical local coordinates. This leads us to the following local description of contact vector fields:

$$
X_{f}=-\sum_{i=1}^{k} \frac{\partial f}{\partial y_{i}} \frac{\partial}{\partial x_{i}}+\left(f-\sum_{i=1}^{k} y_{i} \frac{\partial f}{\partial y_{i}}\right) \frac{\partial}{\partial z}+\sum_{i=1}^{k}\left(\frac{\partial f}{\partial x_{i}}+y_{i} \frac{\partial f}{\partial z}\right) \frac{\partial}{\partial y_{i}} \text {. }
$$

In particular, $X_{1}=\frac{\partial}{\partial z}$.

The Lagrange bracket $[f, g]$ in canonical coordinates has the form:

$$
[f, g]=f \frac{\partial g}{\partial z}-g \frac{\partial f}{\partial z}+\sum_{i=1}^{k}\left(\frac{d}{d x_{i}} \frac{\partial g}{\partial y_{i}}-\frac{d}{d x_{i}} \frac{\partial f}{\partial y_{i}}\right)
$$

where

$$
\frac{d}{d x_{i}}=\frac{\partial}{\partial x_{i}}+y_{i} \frac{\partial}{\partial z}
$$

2.11. Definition. Let $w$ be a contact form on a manifold $M$. A diffeomorphism $\varphi: M \rightarrow M$ is called a contact transformation if

$$
\varphi^{*}(w)=\lambda(w)
$$

for some function $\lambda \in C^{\infty}(M)$.

2.12. For the purpose of local description of contact transformations, consider the model contact space $\mathbb{R}^{2 k+1}$ together with standard contact form (16). Each smooth function $f\left(x_{1}, \ldots, x_{k}\right)$ determines the $k$-dimensional submanifold

$$
L_{f}=\left\{z=f(x), y_{1}=\frac{\partial f}{\partial x_{1}}, \ldots, y_{k}=\frac{\partial f}{\partial x_{k}}\right\} \subset \mathbb{R}^{2 k+1} .
$$

Note that the form $w$ vanishes on $L_{f}$. In addition, suppose $L \subset \mathbb{R}^{2 k+1}$ is a $k$ dimensional integral manifold of the distribution $\mathcal{F}(w)$ such that the functions $x_{1}, \ldots, x_{k}$ can be chosen as coordinates in $L$; then $L$ has the form $L=L_{f}$, where $f=\left.z\right|_{L}$.

Further, note that if a vector field $Y$ of the form

$$
Y=\lambda_{1} \frac{\partial}{\partial y_{1}}+\cdots+\lambda_{k} \frac{\partial}{\partial y_{k}}
$$


is tangent to an integral manifold $L$ at a point $a \in L$, then the form

$$
Y\lrcorner d w=-\left(\lambda_{1} d x_{1}+\cdots+\lambda_{k} d x_{k}\right)
$$

vanishes on $\mathrm{T}_{a} L$, since

$$
\left.(Y\lrcorner d w)\left.\right|_{L}=Y\right\lrcorner\left(\left.d w\right|_{L}\right)=0 .
$$

Therefore, if the forms $d x_{i_{1}}, \ldots, d x_{i_{s}}\left(i_{1}<i_{2}<\cdots<i_{s}\right)$ are linearly independent on $\mathrm{T}_{a} L$, then the forms

$$
d x_{i_{1}}, \ldots, d x_{i_{s}}, d y_{i_{s+1}}, \ldots, d y_{i_{k}}
$$

where $\left(i_{1}, \ldots, i_{s}, i_{s+1}, \ldots, i_{k}\right)$ is a permutation of the indexes $(1, \ldots, k)$, form a basis in $\mathrm{T}_{a}^{*} L$.

In other words, the functions

$$
x_{i_{1}}, \ldots, x_{i_{s}}, y_{i_{s+1}}, \ldots, y_{i_{k}}
$$

corresponding to some permutation of the indexes $(1, \ldots, k)$ can be chosen as local coordinates in a neighborhood of each point $a$ of an integral manifold $L(\operatorname{dim} L=k)$.

Writing $w$ as

$w=d\left(z-x_{i_{s+1}} y_{i_{s+1}}-\cdots-x_{i_{k}} y_{i_{k}}\right)-y_{i_{1}} d x_{i_{1}}-\cdots-y_{i_{s}} d x_{i_{s}}+x_{i_{s+1}} d y_{i_{s+1}}+\cdots+x_{i_{k}} d y_{i_{k}}$

and using the description of manifolds $L_{f}$, we see that an integral manifold $L$ in a neighborhood of a point $a \in L$ can be written as:

$$
\left\{\begin{array}{l}
y_{i_{1}}=\frac{\partial f}{\partial x_{i}}, \ldots, y_{i_{s}}=\frac{\partial f}{\partial x_{i_{s}}}, \\
x_{i_{s+1}}=-\frac{\partial f}{\partial y_{i_{s+1}}}, \ldots, x_{i_{k}}=-\frac{\partial f}{\partial y_{i_{k}}} \\
z=f-y_{i_{s+1}} \frac{\partial f}{\partial y_{i_{s}+1}}-\cdots-y_{i_{k}} \frac{\partial f}{\partial y_{i_{k}}}
\end{array}\right.
$$

for some smooth function $f=f\left(x_{i_{1}}, \ldots, x_{i_{s}}, y_{i_{s+1}}, \ldots, y_{i_{k}}\right)$. The function $f$ is called the generating function of $L$ at the point $a$.

2.13. Let us use the just-obtained local description of integral manifolds of a contact distribution in order to describe contact transformations. For this purpose, we shall establish the association between contact transformations and integral manifolds.

Consider a contact transformation

$$
\varphi:\left(x_{1}, \ldots, x_{k}, y_{1}, \ldots, y_{k}, z\right) \mapsto\left(X_{1}, \ldots, X_{k}, Y_{1}, \ldots, Y_{k}, Z\right)
$$

where $X_{i}=X_{i}(x, y, z), Y_{i}=Y_{i}(x, y, z), Z_{i}=Z_{i}(x, y, z), i=1, \ldots, k$. Condition (19) for $\varphi$ to be contact has the form:

$$
-\lambda w+\varphi^{*}(w)=d Z-\sum_{i=1}^{k} Y_{i} d X_{i}-\lambda d z+\sum_{i=1}^{k} \lambda y_{i} d x_{i}=0
$$


It can be interpreted as follows.

Consider the $(2(2 k+1)+1)$-dimensional manifold $\mathbb{R}^{4 k+3}$ with coordinates $\left(Z, X_{1}, \ldots, X_{k}, Y_{1}, \ldots, Y_{k}, \lambda, z, x_{1}, \ldots, x_{k}, y_{1}, \ldots, y_{k}\right)$ together with the contact 1form

$$
\theta=d Z-\sum_{i=1}^{K}\left(Y_{i} d X_{i}-\lambda y_{i} d x_{i}\right)-\lambda d z .
$$

Each contact transformation $\varphi$ determines the $(2 k+1)$-dimensional submanifold $L^{\varphi}$ in $\mathbb{R}^{4 k+3}$ given by the relations

$$
Z=Z(x, y, z), Y_{i}=Y_{i}(x, y, z), X_{i}=X_{i}(x, y, z), \lambda=\lambda(x, y, z), \quad i=1, \ldots, k \text {. }
$$

From (21) it follows that $L^{\varphi}$ is an integral manifold of the distribution $\mathcal{F}(\theta)$.

The description of integral manifolds in terms of generating functions (see 2.12) enables us to give the complete description of contact transformations of $\mathbb{R}^{2 k+1}$.

2.14. We shall dwell on the case $k=1\left(\mathbb{R}^{3}=J^{1}(\mathbb{R}, \mathbb{R})\right)$, which is most important for us. Let us choose coordinates $(x, y, z)$ such that the Cartan form has the form: $w=d z-y d x$, i.e. $z=u, y=p, x=t$.

The complete description of contact transformations of $J^{1}(\mathbb{R}, \mathbb{R})$ in terms of generating functions can be divided into eight types in accordance with types of functions that can be chosen as coordinates in the manifold $L^{\varphi}$. (By $S$ we denote the corresponding generating function.)

1) $\left(x_{1}, x_{2}, x_{3}\right)$. Coordinates in $L^{\varphi}$ are functions $(X, z, x)$. The generating function $S=S(X, z, x)$ determines the following contact transformation:

$$
Y=\frac{\partial S}{\partial X}, y=-\frac{\partial S}{\partial x} / \frac{\partial S}{\partial z}, Z=S(X, z, x)
$$

2) $\left(y_{1}, x_{2}, x_{3}\right)$. Coordinates in $L^{\varphi}$ are functions $(Y, z, x)$. The generating function $S=S(Y, z, x)$ determines the following contact transformation:

$$
X=-\frac{\partial S}{\partial Y}, y=-\frac{\partial S}{\partial x} / \frac{\partial S}{\partial z}, Z=S-Y \frac{\partial S}{\partial Y} .
$$

3) $\left(x_{1}, y_{2}, x_{3}\right)$. Coordinates in $L^{\varphi}$ are functions $(X, \lambda, x)$. The generating function $S=S(X, \lambda, x)$ determines the following contact transformation:

$$
Y=\frac{\partial S}{\partial X}, z=-\frac{\partial S}{\partial \lambda}, y=-\lambda^{-1} \frac{\partial S}{\partial x}, Z=S-\lambda \frac{\partial S}{\partial \lambda} .
$$

4) $\left(x_{1}, x_{2}, y_{3}\right)$. Coordinates in $L^{\varphi}$ are functions $(X, z,-\lambda y)$. The generating function $S=S(X, z, R)$, where $R=-\lambda y$, determines the following contact transformation:

$$
Y=\frac{\partial S}{\partial X}, x=-\frac{\partial S}{\partial R}, y=-R / \frac{\partial S}{\partial z}, Z=S-R \frac{\partial S}{\partial R} .
$$


5) $\left(x_{1}, y_{2}, y_{3}\right)$. Coordinates in $L^{\varphi}$ are functions $(X, \lambda,-\lambda y)$. The generating function $S=S(X, \lambda, R)$, where $R=-\lambda y$, determines the following contact transformation:

$$
Y=\frac{\partial S}{\partial X}, z=-\frac{\partial S}{\partial \lambda}, x=-\frac{\partial S}{\partial R}, y=-R / \lambda, Z=S-\lambda \frac{\partial S}{\partial \lambda}-R \frac{\partial S}{\partial R} .
$$

6) $\left(y_{1}, x_{2}, y_{3}\right)$. Coordinates in $L^{\varphi}$ are functions $(Y, z,-\lambda y)$. The generating function $S=S(Y, z, R)$, where $R=-\lambda y$, determines the following contact transformation:

$$
X=-\frac{\partial S}{\partial Y}, x=-\frac{\partial S}{\partial R}, y=-R / \frac{\partial S}{\partial z}, Z=S-Y \frac{\partial S}{\partial Y}-R \frac{\partial S}{\partial R} .
$$

7) $\left(y_{1}, y_{2}, x_{3}\right)$. Coordinates in $L^{\varphi}$ are functions $(Y, \lambda, x)$. The generating function $S=S(Y, \lambda, x)$, determines the following contact transformation:

$$
X=-\frac{\partial S}{\partial Y}, z=-\frac{\partial S}{\partial \lambda}, y=-\lambda^{-1} \frac{\partial S}{\partial z}, Z=S-Y \frac{\partial S}{\partial Y}-\lambda \frac{\partial S}{\partial \lambda} .
$$

8) $\left(y_{1}, y_{2}, y_{3}\right)$. Coordinates in $L^{\varphi}$ are functions $(Y, \lambda,-\lambda y)$. The generating function $S=S(Y, \lambda, R)$, where $R=-\lambda y$, determines the following contact transformation:

$$
X=-\frac{\partial S}{\partial Y}, z=-\frac{\partial S}{\partial \lambda}, x=-\frac{\partial S}{\partial R}, y=R \lambda^{-1}, Z=S-Y \frac{\partial S}{\partial Y}-\lambda \frac{\partial S}{\partial \lambda}-R \frac{\partial S}{\partial R} .
$$

Example. Consider the following generating function of type 1:

$$
S(X, z, x)=X z+1 / 2 x^{2} .
$$

Then $S$ determines the contact transformation

$$
\varphi:(x, y, z) \mapsto\left(\frac{-x}{y}, z, 1 / 2 x^{2}-x z / y\right)
$$

Indeed,

$$
\varphi^{*}(w)=d Z-Y d X=d\left(1 / 2 x^{2}-x z / y\right)+z d(x / y)=-x / y(d z-y d x) .
$$

\section{§3. LiE FIELDS AND SYMMETRIES OF CARTAN DISTRIBUTIONS}

Preparatory to considering symmetries of differential equations, consider those transformations of spaces of jets that preserve the Cartan distribution.

3.1. Definition. A diffeomorphism $\varphi$ of the space $J^{k}(\mathbb{R}, M)$ (or $N^{k}$ ) is called a Lie transformation if $\varphi$ is a symmetry of the Cartan distribution.

3.2. Let us consider some examples. Let $M$ be the $n$-dimensional arithmetic space $\mathbb{R}^{n}$ and let $k=1$. Then $J^{1}\left(\mathbb{R}, \mathbb{R}^{n}\right)$ is the arithmetic space $\mathbb{R}^{2 n+1}$ with coordinates $\left(t, u^{1}, \ldots, u^{n}, p_{1}^{1}, \ldots, p_{1}^{n}\right)$. Suppose $\varphi$ is a Lie transformation:

$$
\varphi:\left(t, u^{1}, \ldots, u^{n}, p_{1}^{1}, \ldots, p_{1}^{n}\right) \mapsto\left(T, U^{1}, \ldots, U^{n}, P_{1}^{1}, \ldots, P_{1}^{n}\right),
$$

where $T=T\left(t, u, p_{1}\right), U^{i}=U^{i}\left(t, u, p_{1}\right), P_{1}^{i}=P_{1}^{i}\left(t, u, p_{1}\right)$ for $i=1, \ldots, n$. Then the inverse images $\varphi^{*}\left(\omega_{j}\right)=d U^{i}-P_{1}^{i} d T$ of the Cartan forms $\omega_{j}=d u^{j}-p_{1}^{j} d t$ must vanish on the Cartan distribution. 
Example 1. Transformation of variables. Consider a transformation of dependent and independent variables

$$
\phi:\left(t, u^{1}, \ldots, u^{n}\right) \mapsto\left(T(t), U^{1}(u), \ldots, U^{n}(u)\right)
$$

Define $P_{1}^{j}\left(t, u, p_{1}\right)$ so that the corresponding transformation (1) is a Lie transformation. We have

$$
\phi^{*}\left(\omega_{j}\right)=d U^{j}-P_{1}^{j} d T=\sum_{i} \frac{\partial U^{j}}{\partial u^{i}} d u^{i}-P_{1}^{j} T^{\prime} d t .
$$

These forms must vanish if $\omega_{1}=\cdots=\omega_{n}=0$. Therefore,

$$
\phi^{*}\left(\omega_{j}\right)=\left(\sum_{i} \frac{\partial U^{j}}{\partial u^{i}} p_{1}^{i}-P_{1}^{j} T^{\prime}\right) d t \bmod \left(\omega_{1}, \ldots, \omega_{n}\right) .
$$

Thus, the relations

$$
\left\{\begin{array}{l}
T=T(t) \\
U^{j}=U^{j}\left(t, u^{1}, \ldots, u^{n}\right) \\
P_{1}^{j}=\left(T^{\prime}\right)^{-1} \sum_{i} \frac{\partial U^{j}}{\partial u^{i}} p_{1}^{i}
\end{array}\right.
$$

determine a Lie transformation.

Example 2. Point transformations. Consider an arbitrary transformation of the space $J^{0}\left(\mathbb{R}, \mathbb{R}^{n}\right)=\mathbb{R}^{n+1}$

$$
\phi:\left(t, u^{1}, \ldots, u^{n}\right) \mapsto\left(T, U^{1}, \ldots, U^{n}\right),
$$

where $T=T(t, u), U^{j}=U^{j}(t, u), j=1, \ldots, n$.

Let us prolong $\phi$ to a Lie transformation. As in the previous example, we have

$$
\begin{aligned}
& \phi^{*}\left(\omega_{j}\right)=d U^{j}-P_{1}^{j} d T=\sum_{i} \frac{\partial U^{j}}{\partial u^{i}} d u^{i}+\frac{\partial U^{j}}{\partial t} d t-P_{1}^{j} \frac{\partial T}{\partial t}-\sum_{i} P_{1}^{j} \frac{\partial T}{\partial u^{i}} d u^{i}= \\
& =\left(\sum_{i} \frac{\partial U^{j}}{\partial u^{i}} p_{1}^{i}+\frac{\partial U^{j}}{\partial t}-P_{1}^{j}\left(\sum_{i} \frac{\partial T}{\partial u^{i}} p_{1}^{i}+\frac{\partial T}{\partial t}\right)\right) d t \bmod \left(\omega_{1}, \ldots, \omega_{n}\right) .
\end{aligned}
$$

By $\frac{d}{d t}$ denote the operator of total differentiation with respect to $t$ :

$$
\frac{d}{d t}=\frac{\partial}{\partial t}+p_{1}^{1} \frac{\partial}{\partial u^{1}}+\cdots+p_{1}^{n} \frac{\partial}{\partial u^{n}}
$$

Then from relations $(5)$ and the conditions $\varphi^{*}\left(\omega_{j}\right) \equiv 0 \bmod \left(\omega_{1}, \ldots, \omega_{n}\right)$ it follows that

$$
P_{1}^{j}=\frac{d U^{j}}{d t} / \frac{d T}{d t}, \quad j=1, \ldots, n .
$$


The Lie transformation defined by (4) and (7) is called a point transformation.

Note that transformation of variables (1) is a partial case of point transformations.

3.3. Point transformations have a simple geometrical interpretation. As we saw in chapter 1, every diffeomorphism $\psi: N \rightarrow N$ can be prolonged to the diffeomorphism $\psi^{(k)}: N^{k} \rightarrow N^{k}$ of the space of $k$-jets such that

$$
\psi^{(k)}\left(J_{k}(L)\right)=j_{k}(\psi(L))
$$

for all curves $L$ in the manifold $N$.

Tangent lines to $k$-jets $j_{k}(L)$ generate the Cartan distribution on $N^{k}$. Therefore, prolongations $\psi^{(k)}$ of diffeomorphisms $\psi: N \rightarrow N$ are Lie transformations. We shall call them point transformations.

It is obvious that the definition just given is in accordance with that given in the previous item. It is sufficient to put $N=\mathbb{R} \times \mathbb{R}^{n}$.

3.4. Theorem. Every Lie transformation of the manifold $J^{k}(\mathbb{R}, M)$ (or $N^{k}$ ) is a point transformation under the condition that $\operatorname{dim} M \geqslant 2(\operatorname{dim} N \geqslant 3)$.

We shall first prove a lemma describing the structure of maximal integral manifolds of the Cartan distribution. In order to formulate the lemma, we give the following definition:

Definition. An integral manifold $S \subset J^{k}(\mathbb{R}, M)$ of the Cartan distribution is called infinitesimally maximal if for every point $a \in S$ there does not exist any integral manifold $S^{\prime}$ such that $a \in S^{\prime}, S^{\prime}$ is tangent to $S$ at the point $a$, and $\mathrm{T}_{a} S \varsubsetneqq \mathrm{T}_{a} S^{\prime}$.

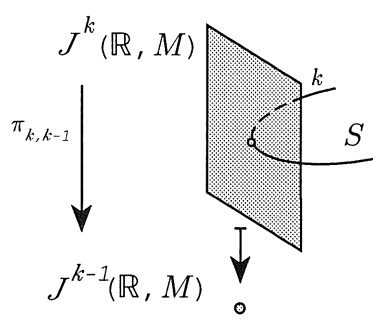

Fig. 19

Lemma. Let $\operatorname{dim} M \geqslant 2$ and $k \geqslant 1$. Then all infinitesimally maximal manifolds in $J^{k}(\mathbb{R}, M)$ are confined to the following ones:

(i) $k$-jets $j_{k}(x)$ of parametrized curves,

(ii) fibres of the projection $\pi_{k, k-1}: J^{k}(\mathbb{R}, M) \rightarrow J^{k-1}(\mathbb{R}, M)$.

Proof of the lemma. Let $S \subset J^{k}(\mathbb{R}, M)$ be a maximal integral manifold of the Cartan distribution. Then

$$
\left.\omega\left(p_{j}^{i}\right)\right|_{S}=0
$$


for all $i=1, \ldots, n$ and $j=0,1, \ldots, k-1$ and therefore

$$
\left.d \omega\left(p_{j}^{i}\right)\right|_{S}=0 .
$$

But

$$
d \omega_{j}^{i}=-d p_{j+1}^{i} \wedge d t .
$$

Therefore, all differential 1-forms $d p_{j+1}^{i}(i=1, \ldots, n ; j=0,1, \ldots, k-1)$ are proportional to $d t$ on $S$. If $\operatorname{dim} S \geqslant 2$, then this is possible if and only if $d t=0$ on $S$. Then from (9) it follows that $d p_{j}^{i}=0$ on $S$ for all $i=1, \ldots, n$ and $j=0,1, \ldots, k-1$. Thus, $S$ lies in a fibre of the projection $\pi_{k, k-1}$ and since $S$ is maximal, we see that $S$ coincides with the fibre. If $\left.d t\right|_{S} \neq 0$ and all forms $d p_{j}^{i}$ are proportional to $d t$, then $S$ is one-dimensional and $t$ can be chosen as a coordinate on $S$ and therefore $S$ is a $k$-jet of a parametrized curve.

Proof of the theorem. Let $\varphi: J^{k}(\mathbb{R}, M) \rightarrow J^{k}(\mathbb{R}, M)$ be an arbitrary Lie transformation. This transformation preserves the class of infinitesimally maximal integral manifolds. Since the dimension of fibres of the projection $\pi_{k, k-1}$ equals $\operatorname{dim} M$, we see that $\varphi$ preserves fibres of $\pi_{k, k-1}$ and therefore induces the transformation $\bar{\varphi}: J^{k-1}(\mathbb{R}, M) \rightarrow J^{k-1}(\mathbb{R}, M)$, satisfying the commutative diagram

$$
\begin{array}{ccc}
J^{k}(\mathbb{R}, M) & \stackrel{\varphi}{\rightarrow} & J^{k}(\mathbb{R}, M) \\
\pi_{k, k-1} \downarrow & & \downarrow \pi_{k, k-1} \\
J^{k-1}(\mathbb{R}, M) & \rightarrow & J^{k-1}(\mathbb{R}, M)
\end{array} .
$$

For a parametrized curve $x: \mathcal{O} \rightarrow M$, by $x_{\varphi}$ denote the curve defined by

$$
j_{k}\left(x_{\varphi}\right)=\varphi\left(j_{k}(x)\right) \text {. }
$$

Since for almost all parametrized curves $x: \mathcal{O} \rightarrow M$

$$
\bar{\varphi}\left(j_{k-1}(x)\right)=\bar{\varphi}\left(\pi_{k, k-1}\left(j_{k}(x)\right)\right)=\pi_{k, k-1}\left(\varphi\left(j_{k}(x)\right)\right)=j_{k-1}\left(x_{\varphi}\right),
$$

we see that $\bar{\varphi}$ is also a Lie transformation.

Note that relations (10) and (11) allow to restore the transformation $\varphi$ starting from $\bar{\varphi}$.

The Lie transformation $\bar{\varphi}$ in its turn determines the Lie transformation

$$
\tilde{\varphi}: J^{k-2}(\mathbb{R}, M) \rightarrow J^{k-2}(\mathbb{R}, M)
$$

and so on. Eventually, we arrive at the point transformation $\psi: M \times \mathbb{R} \rightarrow M \times \mathbb{R}$. Using (11) and (10) in consecutive order, we obtain $\varphi=\psi^{(k)}$.

3.5. Let us now describe Lie transformations of the space $J^{k}(\mathbb{R}, M)$ under the condition that $\operatorname{dim} M=1$. There are two possibilities: either $M=\mathbb{R}$ or $M=\mathrm{S}^{1}$. We shall go into detail only on the first case, for the second one is very similar.

Note that theorem 3.4 is no longer valid for $M=\mathbb{R}$. Indeed, the Cartan distribution on $J^{1}(\mathbb{R}, \mathbb{R})$ is a contact distribution and Lie transformations are contact transformations of $\mathbb{R}^{3}=J^{1}(\mathbb{R}, \mathbb{R})$. The fact that not all contact transformations are point transformations follows from the description of contact transformations of $\mathbb{R}^{3}$ given in the previous section.

Let us make some simple observations. 
Lemma 1. Let $F \in C^{\infty}\left(J^{k}(\mathbb{R}, \mathbb{R})\right)$ be an arbitrary function. Then

$$
d F=\left(\frac{d F}{d t} d t+\frac{\partial F}{\partial p_{k}} d p_{k}\right) \quad \bmod \left(\omega\left(p_{0}\right), \ldots, \omega\left(p_{k-1}\right)\right),
$$

where

$$
\frac{d}{d t}=\frac{\partial}{\partial t}+p_{1} \frac{\partial}{\partial p_{0}}+\cdots+p_{k} \frac{\partial}{\partial p_{k-1}}
$$

is the operator of total differentiation.

Lemma 2. Let $\varphi: J^{k}(\mathbb{R}, \mathbb{R}) \rightarrow J^{k}(\mathbb{R}, \mathbb{R})$ be a Lie transformation of the form:

$$
\varphi:\left(t, p_{0}, \ldots, p_{k}\right) \mapsto\left(T, P_{0}, \ldots, P_{k}\right) .
$$

Then

$$
\frac{d P_{j}}{d t}-P_{j+1} \frac{d T}{d t}=0, \quad \frac{d P_{j}}{d p_{k}}-P_{j+1} \frac{d T}{d p_{k}}=0
$$

for all $j=0, \ldots, k-1$.

Proof. Lemma 2 follows from Lemma 1, while the latter follows from the following relations:

$$
d p_{j}=p_{j+1} d t \quad \bmod \left(\omega\left(p_{0}\right), \ldots, \omega\left(p_{k-1}\right)\right), \quad j=0, \ldots, k-1 .
$$

Let us now show that if $k \geqslant 2$, then $\frac{\partial T}{\partial p_{k}} \equiv 0$. Indeed, if the converse was true, the functions $\left(t, p_{0}, \ldots, p_{k-1}, T\right)$ could be chosen as (local) coordinates in the domain of $J^{k}(\mathbb{R}, \mathbb{R})$ where $\frac{\partial T}{\partial p_{k}} \neq 0$. Then relations (12) would take the form:

$$
\frac{d P_{j}}{d t}=0, \quad P_{j+1}=\frac{\partial P_{j}}{\partial T}, \quad j=0, \ldots, k-1 .
$$

Using the relations

$$
\left[\frac{d}{d t}, \frac{\partial}{\partial T}\right]=-\frac{\partial p_{2}}{\partial T} \frac{\partial}{\partial p_{k-1}}
$$

and

$$
\left[\frac{d}{d t}, \frac{\partial}{\partial p_{j}}\right]=-\frac{\partial}{\partial p_{j-1}}-\frac{\partial p_{2}}{\partial p_{j}} \frac{\partial}{\partial p_{k-1}}, \quad j=0, \ldots, k-2
$$

we obtain

$$
\left\{\begin{array}{l}
{\left[\frac{d}{d t}, \frac{\partial}{\partial T}\right]\left(P_{j}\right)=-\frac{\partial p_{2}}{\partial T} \frac{\partial P_{j}}{\partial p_{k-1}}=0} \\
{\left[\frac{d}{d t}, \frac{\partial}{\partial p_{s}}\right]\left(P_{j}\right)=-\frac{\partial P_{j}}{\partial p_{s-1}}=0} \\
\frac{d P_{j}}{d t}=\frac{\partial P_{j}}{\partial t}=0
\end{array}\right.
$$

which contradicts the fact that the functions $T, P_{0}, \ldots, P_{k}$ are functionally independent. 
Therefore,

$$
\frac{\partial T}{\partial p_{k}}=0, \quad \frac{\partial P_{j}}{\partial p_{k}}=0, \quad j=0,1, \ldots, k-1 .
$$

Thus, a Lie transformation $\varphi$ determines the Lie transformation $\bar{\varphi}: J^{k-1}(\mathbb{R}, \mathbb{R}) \rightarrow$ $J^{k-1}(\mathbb{R}, \mathbb{R})$, satisfying the commutative diagram

$$
\begin{array}{ccc}
J^{k}(\mathbb{R}, \mathbb{R}) & \stackrel{\varphi}{\rightarrow} & J^{k}(\mathbb{R}, \mathbb{R}) \\
\downarrow & & \downarrow \\
J^{k-1}(\mathbb{R}, \mathbb{R}) & \underset{\bar{\varphi}}{\rightarrow} & J^{k-1}(\mathbb{R}, \mathbb{R})
\end{array} .
$$

Proceeding as in the proof of theorem 3.4, we eventually arrive at a contact transformation $\psi: J^{1}(\mathbb{R}, \mathbb{R}) \rightarrow J^{1}(\mathbb{R}, \mathbb{R})$. Moreover, the following result is true:

3.6. Theorem. All Lie transformations of the space $J^{k}(\mathbb{R}, \mathbb{R}), k \geqslant 2$, are prolongations of contact transformations of the space $J^{1}(\mathbb{R}, \mathbb{R})$. In addition, if $\psi: J^{1}(\mathbb{R}, \mathbb{R}) \rightarrow$ $J^{1}(\mathbb{R}, \mathbb{R})$ is a contact transformation of the form

$$
\psi:\left(t, p_{0}, p_{1}\right) \mapsto\left(T, P_{0}, P_{1}\right),
$$

then its prolongation $\psi^{(k)}: J^{k}(\mathbb{R}, \mathbb{R}) \rightarrow J^{k}(\mathbb{R}, \mathbb{R})$ is defined by

$$
\left(t, p_{0}, p_{1}, \ldots, p_{k}\right) \mapsto\left(T, P_{0}, P_{1}, \ldots, P_{k}\right),
$$

where

$$
P_{j+1}=\frac{d P_{j}}{d t} / \frac{d T}{d t}, \quad j=1, \ldots, k-1
$$

Example. The prolongation of the Legendre transformation

$$
\psi:\left(t, p_{0}, p_{1}\right) \mapsto\left(p_{1}, p_{0}-t p_{1},-t\right)
$$

to the Lie transformations of $J^{3}(\mathbb{R}, \mathbb{R})$ has the form:

$$
\psi^{(3)}:\left(t, p_{0}, p_{1}, p_{2}, p_{3}\right) \mapsto\left(p_{1}, p_{0}-t p_{1},-t,-\frac{1}{p_{2}}, \frac{p_{3}}{\left(p_{2}\right)^{3}}\right)
$$

3.7. An arbitrary Lie transformation of the space $J^{k}\left(\mathbb{R}, \mathbb{R}^{n}\right)$ can be given by formulas analogous to formulas (12). Let

$$
\psi:\left(t, p_{0}^{1}, \ldots, p_{0}^{n}\right) \mapsto\left(T, P_{0}^{1}, \ldots, P_{0}^{n}\right)
$$

be a point transformation of the space $J^{0}\left(\mathbb{R}, \mathbb{R}^{n}\right)$ and

$$
\psi^{(k)}:\left(t, p_{j}^{i}\right) \mapsto\left(T, P_{j}^{i}\right), \quad i=1, \ldots, n ; j=0, \ldots, k,
$$

its $k$-th prolongation. In order to find the functions $P_{j}^{i}$, we shall make use of the following result, analogous to lemma 1 from 3.5: 
Lemma. Let $F \in C^{\infty}\left(J^{k}\left(\mathbb{R}, \mathbb{R}^{n}\right)\right)$ be an arbitrary function. Then

$$
d F=\left(\frac{d F}{d t}+\sum_{s} \frac{\partial F}{\partial p_{k}^{s}} d p_{k}^{s}\right) \quad \bmod \left(\omega\left(p_{0}^{1}\right), \ldots, \omega\left(p_{k-1}^{n}\right)\right)
$$

where

$$
\frac{d}{d t}=\frac{\partial}{\partial t}+\sum_{i, j} p_{j+1}^{i} \frac{\partial}{\partial p_{j}^{i}}
$$

Since

$$
\frac{\partial T}{\partial p_{k}^{s}}=0, \quad \frac{\partial P_{j}^{i}}{\partial p_{k}^{s}}=0, \quad s, i=1, \ldots, n ; j=0,1, \ldots, k-1,
$$

from the lemma we obtain

$$
\begin{gathered}
\left(\psi^{(k)}\right)^{*}\left(\omega\left(p_{j}^{i}\right)\right)=d P_{j}^{i}-P_{j+1}^{i} d T= \\
=\left(\frac{d P_{j}^{i}}{d t}-P_{j+1}^{i} \frac{d T}{d t}\right) d t \bmod \left(\omega\left(p_{0}^{1}\right), \ldots, \omega\left(p_{k-1}^{n}\right)\right) .
\end{gathered}
$$

Therefore

$$
P_{j+1}^{i}=\frac{d P_{j}^{i}}{d t} / \frac{d T}{d t} .
$$

Starting from the functions $P_{0}^{i}=P_{0}^{i}\left(t, p_{0}^{1}, \ldots, p_{0}^{n}\right)$ and using (16), we can find Lie transformation (14).

3.8. Let us now consider infinitesimal symmetries of the Cartan distribution.

Definition. A vector field $X$ on the manifold of $k$-jets of curves is called a Lie field if $X$ is an infinitesimal symmetry of the Cartan distribution.

Description of Lie vector fields can be divided into two parts, just as the description of Lie transformations.

Let $\operatorname{dim} M \geqslant 2$. Suppose $X$ is a vector field on the manifold $J^{0}(\mathbb{R}, M)=\mathbb{R} \times M$ and $\left\{\psi_{t}\right\}$ is the one-parameter group of translations along $X$. Then its prolongation $\left\{\psi_{t}^{(k)}: J^{k}(\mathbb{R}, M) \rightarrow J^{k}(\mathbb{R}, M)\right\}$ is also a one-parameter group. By $X^{(k)}$ denote the vector field on $J^{k}(\mathbb{R}, M)$ corresponding to the group $\left\{\psi_{t}^{(k)}\right\}$.

Definition. The vector field $X^{(k)}$ is called the $k$-th prolongation of the vector field $X$.

We shall now describe the procedure of prolongation of a vector field in coordinates, using the model $M=\mathbb{R}^{n}$.

Every vector field $X$ on $J^{0}\left(\mathbb{R}, \mathbb{R}^{n}\right)$ can be written as

$$
X=A\left(t, p_{0}^{i}\right) \frac{\partial}{\partial t}+B^{1}\left(t, p_{0}^{i}\right) \frac{\partial}{\partial p_{0}^{1}}+\cdots+B^{n}\left(t, p_{0}^{i}\right) \frac{\partial}{\partial p_{0}^{n}} .
$$


Then the $k$-th prolongation of $X$ has the form:

$$
X^{(k)}=A\left(t, p_{0}^{i}\right) \frac{\partial}{\partial t}+\sum_{i, j} B_{j}^{i} \frac{\partial}{\partial p_{j}^{i}}
$$

where $i=1, \ldots, n, j=0, \ldots, k$, and $B_{0}^{i}=B^{i}$.

Now find the functions $B_{j}^{i}$. Let $\left\{\varphi_{s}\right\}$ be the one-parameter group of transformations along $X$ :

$$
\varphi_{s}:\left(t, p_{0}^{i}\right) \mapsto\left(T\left(s, t, p_{0}^{l}\right), P_{0}^{i}\left(s, t, p_{0}^{l}\right)\right),
$$

and $\left\{\varphi_{s}^{(k)}\right\}$ the $k$-th prolongation of the group $\left\{\varphi_{s}\right\}$ :

$$
\varphi_{s}^{(k)}:\left(t, p_{j}^{i}\right) \mapsto\left(T\left(s, t, p_{0}^{l}\right), P_{j}^{i}\left(s, t, p_{m}^{l}\right)\right) .
$$

Since

$$
\begin{gathered}
T\left(s, t, p_{0}^{l}\right)=t+s A+\mathrm{o}(s), \\
P_{j}^{i}\left(s, t, p_{m}^{l}\right)=p_{j}^{i}+s B_{j}^{i}+\mathrm{o}(s)
\end{gathered}
$$

and therefore

$$
\left.\frac{d T}{d t}\right|_{s=0}=1,\left.\quad \frac{d P_{j}^{i}}{d t}\right|_{s=0}=P_{j+1}^{i}
$$

using (16), we obtain

$$
B_{j+1}^{i}=\left.\frac{\partial}{\partial s}\right|_{s=0}\left(P_{j+1}^{i}\left(s, t, p_{m}^{l}\right)\right)=\left.\frac{\partial}{\partial s}\right|_{s=0}\left(\frac{d P_{j}^{i}}{d t} / \frac{d T}{d t}\right)=\frac{d B_{j}^{i}}{d t}-p_{j+1}^{i} \frac{d A}{d t} .
$$

Thus, we obtain the following recursion equations for the functions $B_{j}^{i}$ :

$$
\left\{\begin{array}{l}
B_{j+1}^{i}=\frac{d B_{j}^{i}}{d t}-p_{j+1}^{i} \frac{d A}{d t} \\
B_{0}^{i}=B^{i}
\end{array}\right.
$$

where $i=1, \ldots, n, j=0,1, \ldots, k-1$.

3.9. Example. For the first prolongation $X^{(1)}$ we have

$$
\begin{gathered}
B_{1}^{i}=\frac{d B^{i}}{d t}-p_{1}^{i} \frac{d A}{d t}=\left(\frac{d B^{i}}{d t}+\sum_{l} p_{1}^{l} \frac{\partial B^{i}}{\partial p_{0}^{l}}\right)-p_{1}^{i}\left(\frac{d A}{d t}+\sum_{l} p_{1}^{l} \frac{\partial A}{\partial p_{0}^{l}}\right)= \\
=\frac{\partial B^{i}}{\partial t}+\left(\sum_{l} p_{1}^{l} \frac{\partial B^{l}}{\partial p_{0}^{l}}-p_{1}^{i} \frac{d A}{d t}\right)-\sum_{l} p_{1}^{i} p_{1}^{l} \frac{\partial A}{\partial p_{0}^{l}}
\end{gathered}
$$

3.10. Now let $M=\mathbb{R}$. Suppose $X=X_{f}$ is a contact vector field on the manifold $J^{1}(\mathbb{R}, \mathbb{R})$ and $\left\{\psi_{s}: J^{1}(\mathbb{R}, \mathbb{R}) \rightarrow J^{1}(\mathbb{R}, \mathbb{R})\right\}$ the corresponding one-parameter group of 
contact transformations. By $X_{f}^{(k)}$ denote the vector field on $J^{k}(\mathbb{R}, \mathbb{R})$ corresponding to the prolongation $\left\{\psi_{s}^{(k)}\right\}$ of the group $\left\{\psi_{s}\right\}$. The field $X_{f}^{(k)}$ is called the $(k-1)$-th prolongation of $X_{f}$.

Let us find the explicit form of $X_{f}^{(k)}$. Let $X_{f}$ have the form

$$
X_{f}=-\frac{\partial f}{\partial p_{1}} \frac{\partial}{\partial t}+\left(f-p_{1} \frac{\partial f}{\partial p_{1}}\right) \frac{\partial}{\partial p_{0}}+\left(\frac{\partial f}{\partial t}+p_{1} \frac{\partial f}{\partial p_{0}}\right) \frac{\partial}{\partial p_{1}}
$$

and let

$$
\varphi_{s}:\left(t, p_{0}, p_{1}\right) \mapsto\left(T\left(s, t, p_{0}, p_{1}\right), P_{0}\left(s, t, p_{0}, p_{1}\right), P_{1}\left(s, t, p_{0}, p_{1}\right)\right)
$$

be the corresponding one-parameter group, so that

$$
\begin{aligned}
& T=t-\frac{\partial f}{\partial p_{1}} s+o(s) \\
& P_{0}=p_{0}+\left(f-p_{1} \frac{\partial f}{\partial p_{1}}\right) s+o(s), \\
& P_{1}=p_{1}+\left(\frac{\partial f}{\partial t}+p_{1} \frac{\partial f}{\partial p_{0}}\right) s+o(s) .
\end{aligned}
$$

Let $X_{f}^{(k)}$ have the form:

$$
X_{f}^{(k)}=-\frac{\partial f}{\partial p_{1}} \frac{\partial}{\partial t}+\left(f-p_{1} \frac{\partial f}{\partial p_{1}}\right) \frac{\partial}{\partial p_{0}}+\left(\frac{\partial f}{\partial t}+p_{1} \frac{\partial f}{\partial p_{0}}\right) \frac{\partial}{\partial p_{1}}+\sum_{j=2}^{k} B_{j} \frac{\partial}{\partial p_{j}} .
$$

The components of the corresponding prolongation

$$
\varphi_{s}^{(k)}:\left(t, p_{0}, \ldots, p_{k}\right) \mapsto\left(T, P_{0}, \ldots, P_{k}\right)
$$

have the form

$$
P_{j}=p_{j}+B_{j} s+\mathrm{o}(s), \quad j=2, \ldots, k,
$$

and can be found from recursive relations (13). Therefore,

$$
B_{j+1}=\left.\frac{\partial}{\partial s}\right|_{s=0}\left(P_{j+1}\right)=\left.\frac{\partial}{\partial s}\right|_{s=0}\left(\frac{d P_{j}}{d t} / \frac{d T}{d t}\right)=\frac{d B_{j}}{d t}+p_{j+1} \frac{d}{d t}\left(\frac{\partial f}{\partial p_{1}}\right) .
$$

Finally,

$$
B_{j+1}=\frac{d B_{j}}{d t}+p_{j+1} \frac{d}{d t}\left(\frac{\partial f}{\partial p_{1}}\right), \quad j=1, \ldots, k-1
$$

and

$$
B_{1}=\frac{\partial f}{\partial t}+p_{1} \frac{\partial f}{\partial p_{0}}
$$


3.11. Example. For the first prolongation $X_{f}^{(2)}$ of the contact vector field $X_{f}$, we have

$$
\begin{gathered}
B_{2}=\frac{d}{d t}\left(\frac{\partial f}{\partial t}+p_{1} \frac{\partial f}{\partial p_{0}}\right)+p_{2} \frac{d}{d t}\left(\frac{\partial f}{\partial p_{1}}\right)= \\
=\left(\frac{\partial^{2} f}{\partial t^{2}}+2 p_{1} \frac{\partial^{2} f}{\partial t \partial p_{0}}+2 p_{2} \frac{\partial^{2} f}{\partial t \partial p_{1}}+p_{2} \frac{\partial f}{\partial p_{0}}+p_{1}^{2} \frac{\partial^{2} f}{\partial p_{0}^{2}}+2 p_{1} p_{2} \frac{\partial^{2} f}{\partial p_{0} \partial p_{1}}+p_{2}^{2} \frac{\partial^{2} f}{\partial p_{1}^{2}}\right) .
\end{gathered}
$$

3.12. Examples. In conclusion, let us give several examples of the most useful contact vector fields.

1. Point vector fields are contact vector fields corresponding to functions linear with respect to $p_{1}$ :

$$
f=B\left(t, p_{0}\right)-p_{1} A\left(t, p_{0}\right)
$$

Here

$$
X_{f}=A \frac{\partial}{\partial t}+B \frac{\partial}{\partial p_{0}}+\left(\frac{\partial B}{\partial t}+p_{1}\left(\frac{\partial B}{\partial p_{0}}-\frac{\partial A}{\partial t}\right)-p_{1}^{2} \frac{\partial A}{\partial p_{0}}\right) \frac{\partial}{\partial p_{1}}
$$

The first prolongation of $X_{f}$ has the form:

$$
\begin{gathered}
X_{f}^{(2)}=A \frac{\partial}{\partial t}+B \frac{\partial}{\partial p_{0}}+\left(\frac{\partial B}{\partial t}+p_{1}\left(\frac{\partial B}{\partial p_{0}}-\frac{\partial A}{\partial t}\right)-p_{1}^{2} \frac{\partial A}{\partial p_{0}}\right) \frac{\partial}{\partial p_{1}}+ \\
+\left(\frac{\partial^{2} B}{\partial t^{2}}-p_{1} \frac{\partial^{2} A}{\partial t^{2}}-2 p_{2} \frac{\partial A}{\partial t}+2 p_{1} \frac{\partial^{2} B}{\partial t \partial p_{0}}+p_{2} \frac{\partial B}{\partial p_{0}}-2 p_{1}^{2} \frac{\partial^{2} A}{\partial t \partial p_{0}}+p_{1} p_{2} \frac{\partial A}{\partial p_{0}}+\right. \\
\left.+p_{1}^{2} \frac{\partial^{2} B}{\partial p_{0}^{2}}-p_{1}^{3} \frac{\partial^{2} A}{\partial p_{0}^{2}}\right) \frac{\partial}{\partial p_{2}}
\end{gathered}
$$

2. Translations with respect to $t$.

The vector field $X=\frac{\partial}{\partial t}$ corresponds to translations along the $t$-axis: $\left(t, p_{0}\right) \mapsto$ $\left(t+s, p_{0}\right)$. The corresponding generating function has the form:

$$
f=-p_{1}
$$

The first prolongation (as well as all subsequent ones) has the form:

$$
X_{f}^{(2)}=\frac{\partial}{\partial t}
$$

3. Translations with respect to $p_{0}$.

The vector field $X=\frac{\partial}{\partial p_{0}}$ corresponds to translations along the $p_{0}$-axis: $\left(t, p_{0}\right) \mapsto$ $\left(t, p_{0}+s\right)$. The corresponding generating function has the form:

$$
f=1 \text {. }
$$


The first prolongation (as well as all subsequent ones) has the form:

$$
X_{f}^{(2)}=\frac{\partial}{\partial p_{0}}
$$

4. Scaling (or similitude) transformations have the form:

$$
\left(t, p_{0}\right) \mapsto\left(\mathrm{e}^{\alpha s} t, \mathrm{e}^{\beta s} p_{0}\right)
$$

where $\alpha$ and $\beta$ are constant numbers. The corresponding vector field and generating function have the form:

$$
\begin{gathered}
X=\alpha t \frac{\partial}{\partial t}+\beta p_{0} \frac{\partial}{\partial p_{0}}, \\
f=\beta p_{0}-\alpha p_{1} t .
\end{gathered}
$$

The first prolongation has the form:

$$
X_{f}^{(2)}=\alpha t \frac{\partial}{\partial t}+\beta p_{0} \frac{\partial}{\partial p_{0}}+(\beta-\alpha) p_{1} \frac{\partial}{\partial p_{1}}+(\beta-2 \alpha) p_{2} \frac{\partial}{\partial p_{2}} .
$$

\section{§4. Symmetries of Differential EQUATIONS}

4.1. We can look at a system of ordinary differential equations of order $k$ from two standpoints:

i) external standpoint: it is a submanifold $\mathcal{E}$ in the space $J^{k}(\mathbb{R}, M)$, where $J^{k}(\mathbb{R}, M)$ is supplied with the Cartan distribution;

ii) internal standpoint: it is a manifold $\mathcal{E}$ supplied with the Cartan distribution $C(\mathcal{E})$ of dimension 1

From now on, we shall assume that $\mathcal{E}$ is a regular system of differential equations; in other words, at each point $a \in \mathcal{O}$, the subspaces $\mathrm{T}_{a} \mathcal{E}$ and $C(a)$ are transversal and

$$
C(\mathcal{E})(a)=C(a) \cap \mathrm{T}_{a} \mathcal{E}
$$

In accordance with these standpoints, we can consider two kinds of symmetries.

Definition. Let $\mathcal{E}$ be a regular system of ordinary differential equations of order $k$.

(1) An internal symmetry of the system $\mathcal{E}$ is a transformation of the manifold $\mathcal{E}$ preserving the Cartan distribution $C(\mathcal{E})$.

(2) An external symmetry of the system $\mathcal{E}$ is a diffeomorphism $\varphi$ of the manifold $J^{k}(\mathbb{R}, M)$ such that

a) $\varphi$ is a symmetry of the Cartan distribution on $J^{k}(\mathbb{R}, M)$,

b) $\varphi$ maps the manifold $\mathcal{E}$ in itself.

4.2. We are interested mainly in infinitesimal analogues of the definitions above. 


\section{Definition.}

(1) An internal (infinitesimal) symmetry of the system $\mathcal{E}$ is a shuffling symmetry of the distribution $C(\mathcal{E})$.

(2) A Lie vector field on $J^{k}(\mathbb{R}, M)$ is called an external or classical (infinitesimal) symmetry of the system $\mathcal{E}$ if it is tangent to the submanifold $\mathcal{E} \subset J^{k}(\mathbb{R}, M)$.

In the sequel by symmetries we shall always mean infinitesimal symmetries.

By $\operatorname{sym}_{c}(\mathcal{E})$ denote the Lie algebra of classical symmetries and by $\operatorname{shuf}(\mathcal{E})$ the Lie algebra of internal symmetries.

It is obvious that restriction of the classical symmetry to the submanifold $\mathcal{E}$ gives an internal symmetry of $\mathcal{E}$. Thus, we obtain the homomorphism of Lie algebras

$$
\varkappa: \operatorname{sym}_{c}(\mathcal{E}) \rightarrow \operatorname{shuf}(\mathcal{E}) .
$$

Elements of ker $\varkappa$ are called characteristics of the system $\mathcal{E}$.

4.3. Example 1. The ordinary differential equation

$$
y^{\prime}=F(t, y)
$$

considered from the external standpoint, is the surface $\mathcal{E}$ in $J^{1}(\mathbb{R}, \mathbb{R})=\mathbb{R}^{3}$ given by

$$
p_{1}=F\left(t, p_{0}\right) \text {. }
$$

From the internal standpoint, differential equation (1) is the distribution on $\mathcal{E}$ given by the differential 1-form

$$
\omega=d p_{0}-F\left(t, p_{0}\right) d t
$$

where $\left(t, p_{0}\right)$ are coordinates on the surface $\mathcal{E}$.

A classical symmetry of $\mathcal{E}$ is a Lie vector field or a contact field of the form

$$
X_{f}=-\frac{\partial f}{\partial p_{1}} \frac{\partial}{\partial t}+\left(f-p_{1} \frac{\partial f}{\partial p_{1}}\right) \frac{\partial}{\partial p_{0}}+\left(\frac{\partial f}{\partial t}+p_{1} \frac{\partial f}{\partial p_{0}}\right) \frac{\partial}{\partial p_{1}}
$$

tangent to the surface $\mathcal{E}$. The condition that $X_{f}$ be tangent to $\mathcal{E}$ means that

$$
X_{f}\left(p_{1}-F\left(t, p_{0}\right)\right)=\frac{\partial f}{\partial t}+p_{1} \frac{\partial f}{\partial p_{0}}+\frac{\partial f}{\partial p_{1}} \frac{\partial F}{\partial t}-\left(f-p_{1} \frac{\partial f}{\partial p_{1}}\right) \frac{\partial F}{\partial p_{0}}=0
$$

on the surface $\mathcal{E}$.

Note that condition (4) is automatically satisfied for functions $f$ of the form:

$$
f=\lambda\left(t, p_{0}, p_{1}\right)\left(p_{1}-F\left(t, p_{0}\right)\right) .
$$

Since every function $f\left(t, p_{0}, p_{1}\right)$ can be written as

$$
f\left(t, p_{0}, p_{1}\right)=g\left(t, p_{0}\right)+\lambda\left(t, p_{0}, p_{1}\right)\left(p_{1}-F\left(t, p_{0}\right)\right)
$$


using the fact that equation (4) is linear with respect to $f$, we obtain the equation for $g\left(t, p_{0}\right)$ describing classical symmetries of equation (1):

$$
\frac{\partial g}{\partial t}+F\left(t, p_{0}\right) \frac{\partial g}{\partial p_{0}}-g\left(t, p_{0}\right) \frac{\partial F}{\partial p_{0}}=0
$$

This equation coincides with equation 1.16.(22) describing shuffling symmetries of the distribution $\mathcal{F}(\omega)$ on $\mathcal{E}$. Thus, the homomorphism $\varkappa$ has the form:

$$
\varkappa: X_{f} \mapsto g\left(t, p_{0}\right) \frac{\partial}{\partial p_{0}} .
$$

The module of characteristics is generated by the contact vector field $X_{f_{0}}$, where $f_{0}=-p_{1}+F\left(t, p_{0}\right)$. The restriction of $X_{f_{0}}$ to $\mathcal{E}$ is the already-known characteristic vector field of equation (1) (see 1.16):

$$
\frac{\partial}{\partial t}+F\left(t, p_{0}\right) \frac{\partial}{\partial p_{0}}
$$

Note that in this case $\varkappa$ is an epimorphism.

Example 2. Let us consider several most popular contact symmetries of equation (1). (See also 3.12.)

a) Suppose that equation (1) is invariant under translations along the $t$-axis. The corresponding symmetry is the classical symmetry with the generating function $f=$ $-p_{1}$. Substituting $f$ into equation (4), we see that $\mathcal{E}$ is invariant under translations along the $t$-axis if

$$
\frac{\partial F}{\partial t}=0
$$

b) Suppose that equation (1) is invariant under translations along the $p_{0}$-axis. The corresponding symmetry is the classical symmetry with the generating function $f=1$. From (4) it follows that in this case the function $F$ must satisfy the following condition:

$$
\frac{\partial F}{\partial p_{0}}=0
$$

c) Suppose that equation (1) is invariant under scaling transformations. The corresponding symmetry is the classical symmetry with the generating function $f=$ $\beta p_{0}-\alpha p_{1} t$, where $\alpha$ and $\beta$ are similitude exponents. Substituting $f$ into equation (4), we see that the function $F$ must satisfy the following condition:

$$
\alpha t \frac{\partial F}{\partial t}+\beta p_{0} \frac{\partial F}{\partial p_{0}}=(\alpha-\beta) F .
$$

This means that $F$ is a homogeneous function:

$$
F\left(\mathrm{e}^{\alpha s} t, \mathrm{e}^{\beta s} p_{0}\right)=\mathrm{e}^{(\alpha-\beta)} F\left(t, p_{0}\right) .
$$


Therefore, $F$ can be written as

$$
F\left(t, p_{0}\right)=t^{(\alpha-\beta) / \alpha} h\left(p_{0} t^{-\beta / \alpha}\right)
$$

for some function $h=h(x)$.

d) Generating functions corresponding to point symmetries have the form $f=$ $B\left(t, p_{0}\right)-p_{1} A\left(t, p_{0}\right)$. If we write it in form (5), we obtain

$$
g\left(t, p_{0}\right)=B\left(t, p_{0}\right)-F\left(t, p_{0}\right) A\left(t, p_{0}\right)
$$

and

$$
F\left(t, p_{0}\right)=g\left(t, p_{0}\right) \int g^{-2} \frac{\partial g}{\partial t} d p_{0}
$$

This formula allows to give an explicit form of first-order differential equations having point symmetries. For example, if a symmetry has the generating function $g=\mathrm{e}^{-t p_{0}}$, the corresponding differential equation has the form:

$$
t y^{\prime}=\left(c-\mathrm{e}^{t y}\right)
$$

4.4. Let $k \geqslant 2$ and let $\mathcal{E} \subset J^{k}(\mathbb{R}, \mathbb{R})$ be a $k$-th order differential equation with respect to a function $y: \mathbb{R} \rightarrow \mathbb{R}$. In addition suppose that $\mathcal{E}$ is solvable with respect to the derivative of the highest order:

$$
y^{(k)}=F\left(t, y, y^{\prime}, \ldots, y^{(k-1)}\right)
$$

Let us describe symmetries of equation (10). We shall start with internal ones.

The manifold $\mathcal{E}$ is given by

$$
p_{k}=F\left(t, p_{0}, \ldots, p_{k-1}\right) .
$$

The Cartan distribution on $\mathcal{E}$ can be given by the differential 1-forms

$$
\begin{aligned}
\omega_{0} & =d p_{0}-p_{1} d t, \ldots, \omega_{k-2}=d p_{k-2}-p_{k-1} d t \\
\omega_{k-1} & =d p_{k-1}-F(t, p) d t .
\end{aligned}
$$

In this case the characteristic distribution coincides with the Cartan distribution and therefore is generated by a vector field $\mathcal{D}$. The projection of $\mathcal{D}$ on the $t$-axis does not vanish. Let the functions $t, p_{0}, \ldots, p_{k-1}$ be coordinates on $\mathcal{E}$, then the field $\mathcal{D}$ can be written as

$$
\mathcal{D}=\frac{\partial}{\partial t}+a_{0} \frac{\partial}{\partial p_{0}}+\cdots+a_{k-1} \frac{\partial}{\partial p_{k-1}} .
$$

Since $\mathcal{D}$ is characteristic, we have

$$
\omega_{0}(\mathcal{D})=-p_{1}+a_{0}=0, \ldots, \omega_{k-2}(\mathcal{D})=-p_{k-1}+a_{k-2}=0,
$$




$$
\omega_{k-1}(\mathcal{D})=-F+a_{k-1}=0 .
$$

Therefore, the field $\mathcal{D}$ has the form

$$
\mathcal{D}=\frac{\partial}{\partial t}+p_{1} \frac{\partial}{\partial p_{0}}+\cdots+p_{k-1} \frac{\partial}{\partial p_{k-2}}+F \frac{\partial}{\partial p_{k-1}}
$$

and coincides with the operator of total differentiation of the equation.

For every shuffling symmetry we can choose a representative of the form:

$$
X=\alpha_{-1} \frac{\partial}{\partial t}+\alpha_{0} \frac{\partial}{\partial p_{0}}+\cdots+\alpha_{k-1} \frac{\partial}{\partial p_{k-1}}
$$

where $\alpha_{-1}, \ldots, \alpha_{k-1}$ are functions of $\left(t, p_{0}, \ldots, p_{k-1}\right)$. Since shuffling symmetries are considered up to summands of the form $f\left(t, p_{0}, \ldots, p_{k-1}\right) \mathcal{D}$, we see that in each coset there exists a unique representative of the form:

$$
X=\alpha_{0} \frac{\partial}{\partial p_{0}}+\alpha_{1} \frac{\partial}{\partial p_{1}}+\cdots+\alpha_{k-1} \frac{\partial}{\partial p_{k-1}} .
$$

Consider the Lie derivative with respect to $X$ at the forms $\omega_{i}, i=0, \ldots, k-2$ :

$$
L_{X}\left(\omega_{i}\right)=L_{X}\left(d p_{i}-p_{i+1} d t\right)=d \alpha_{i}-\alpha_{i+1} d t
$$

Since the relation

$$
d h=\mathcal{D}(h) d t \quad \bmod \left(\omega_{0}, \ldots, \omega_{k-1}\right)
$$

is valid for an arbitrary function $h=h\left(t, p_{0}, \ldots, p_{k-1}\right)$, we obtain

$$
L_{X}\left(\omega_{i}\right)=\left(\mathcal{D}\left(\alpha_{i}\right)-\alpha_{i+1}\right) d t \bmod \left(\omega_{0}, \ldots, \omega_{k-1}\right),
$$

so that

$$
\alpha_{1}=\mathcal{D}\left(\alpha_{0}\right), \alpha_{2}=\mathcal{D}\left(\alpha_{1}\right)=\mathcal{D}^{2}\left(\alpha_{0}\right), \ldots, \alpha_{k-1}=\mathcal{D}\left(\alpha_{k-2}\right)=\mathcal{D}^{k-1}\left(\alpha_{0}\right)
$$

Let $f$ denote the function $\alpha_{0}$. Then every internal symmetry of a $k$-th order differential equation is determined by $f$ and has the form:

$$
X_{f}=f \frac{\partial}{\partial p_{0}}+\mathcal{D}(f) \frac{\partial}{\partial p_{1}}+\cdots+\mathcal{D}^{k-1}(f) \frac{\partial}{\partial p_{k-1}} \text {. }
$$

The function $f$ is called the generating function of the symmetry $X_{f}$. Note that there is an analogy with the case of contact vector fields; namely, the generating function of $X_{f}$ can be found from

$$
f=\omega_{0}\left(X_{f}\right)
$$


We have so far used only the forms $\omega_{0}, \ldots, \omega_{k-2}$. For the form $\omega_{k-1}$ we have

$$
L_{X}\left(\omega_{k-1}\right)=\left(\mathcal{D}\left(\alpha_{k-1}\right)-X(F)\right) d t \bmod \left(\omega_{0}, \ldots, \omega_{k-1}\right) \text {. }
$$

Using (13), we obtain the equation with respect to $f$ :

$$
L_{F}(f)=\mathcal{D}^{k}(f)-\frac{\partial F}{\partial p_{k-1}} \mathcal{D}^{k-1}(f)-\cdots-\frac{\partial F}{\partial p_{1}} \mathcal{D}(f)-\frac{\partial F}{\partial p_{0}} f=0 .
$$

Equation (15) is called the Lie equation, while the operator

$$
L_{F}=\mathcal{D}^{k}-\frac{\partial F}{\partial p_{k-1}} \mathcal{D}^{k-1}-\cdots-\frac{\partial F}{\partial p_{1}} \mathcal{D}-\frac{\partial F}{\partial p_{0}}
$$

is called the Lie operator.

The Lie equation is linear with respect to $f$. This means that its solutions form a vector space over the field $\mathbb{R}$. Moreover, this space can be turned into a Lie algebra if we define a bracket $(f, g) \mapsto[f, g]$ on the solution space by

$$
\left[X_{f}, X_{g}\right]=X_{[f, g]} .
$$

From (14) it follows that

$$
[f, g]=\sum_{i=0}^{k-1}\left(\mathcal{D}^{i}(f) \frac{\partial g}{\partial p_{i}}-\mathcal{D}^{i}(g) \frac{\partial f}{\partial p_{i}}\right) .
$$

It is immediate from definition (17) that the bracket [,] truly turns the solution space of equation (15) into a Lie algebra. The bracket $[f, g]$ is called the Jacobi bracket of functions $f$ and $g$.

Summarizing all that has been said in this item, we obtain the following result:

Theorem. Every internal symmetry of differential equation (10) has the form $X_{f}$ (13) for some solution $f$ of the Lie equation. The solution space of the Lie equation is a Lie algebra with respect to Jacobi bracket. A symmetry $X_{f}$ is the image of some classical symmetry by the mapping $\varkappa$ if and only if its generating function $f$ is independent of $p_{2}, \ldots, p_{k-1}$, i.e. $f=f\left(t, p_{0}, p_{1}\right)$. In this case, the internal symmetry $X_{f}$ is the image of the contact vector field with the same generating function.

4.5. Let us now give several examples of how to find symmetries of second-order differential equations.

We shall start with equations of the form:

$$
y^{\prime \prime}=y^{\prime}+\varphi(y)
$$

Since these equations are invariant under translations along the $t$-axis, they always have the symmetry with generating function $f=p_{1}$. We shall find out when they have at least two point symmetries. 
Generating functions of point symmetries have the form

$$
f=B\left(t, p_{0}\right)-p_{1} A\left(t, p_{0}\right)
$$

and satisfy the Lie equation:

$$
\mathcal{D}^{2}(f)-\mathcal{D}(f)-\varphi^{\prime}(f)=0,
$$

where

$$
\mathcal{D}=\frac{\partial}{\partial t}+p_{1} \frac{\partial}{\partial p_{0}}+\left(p_{1}+\varphi\right) \frac{\partial}{\partial p_{1}} .
$$

Substituting (20) into equation (21), we obtain

$$
\begin{gathered}
-\frac{\partial^{2} A}{\partial p_{0}^{2}} p_{1}^{3}+\left(\frac{\partial^{2} B}{\partial p_{0}^{2}}-2 \frac{\partial^{2} A}{\partial t \partial p_{0}}-2 \frac{\partial A}{\partial p_{0}}\right) p_{1}^{2}+\left(-3 \varphi \frac{\partial A}{\partial p_{0}}-\frac{\partial A}{\partial t}-\frac{\partial^{2} A}{\partial t^{2}}+2 \frac{\partial^{2} B}{\partial t \partial p_{0}}\right) p_{1}+ \\
+\left(-2 \varphi \frac{\partial A}{\partial t}+\varphi \frac{\partial B}{\partial p_{0}}+\frac{\partial^{2} B}{\partial t^{2}}-\frac{\partial B}{\partial t}-\varphi^{\prime} B\right)=0 .
\end{gathered}
$$

The left-hand side of the equation obtained is a polynomial in $p_{1}$. Therefore,

$$
\left\{\begin{array}{l}
\frac{\partial^{2} A}{\partial p_{0}^{2}}=0 \\
\frac{\partial^{2} B}{\partial p_{0}^{2}}-2 \frac{\partial^{2} A}{\partial t \partial p_{0}}-2 \frac{\partial A}{\partial p_{0}}=0 \\
-3 \varphi \frac{\partial A}{\partial p_{0}}-\frac{\partial A}{\partial t}-\frac{\partial^{2} A}{\partial t^{2}}+2 \frac{\partial^{2} B}{\partial t \partial p_{0}}=0 \\
-2 \varphi \frac{\partial A}{\partial t}+\varphi \frac{\partial B}{\partial p_{0}}+\frac{\partial^{2} B}{\partial t^{2}}-\frac{\partial B}{\partial t}-\varphi^{\prime} B=0
\end{array}\right.
$$

From the first equation of the system it follows that

$$
A\left(t, p_{0}\right)=a(t) p_{0}+b(t) .
$$

Substituting this expression into the second equation of the system, we obtain

$$
\frac{\partial^{2} B}{\partial p_{0}^{2}}=2 a+2 a^{\prime}
$$

or

$$
B\left(t, p_{0}\right)=\left(a+a^{\prime}\right) p_{0}^{2}+c(t) p_{0}+d(t)
$$

for some functions $c(t)$ and $d(t)$. Then the third equation of system (22) reduces to

$$
3 a \varphi=3\left(a^{\prime}+a^{\prime \prime}\right) p_{0}-b^{\prime \prime}-b^{\prime}-2 c^{\prime} .
$$

Assuming that the function $\varphi=\varphi\left(p_{0}\right)$ is nonlinear (the linear case will be considered later), we obtain

$$
\begin{aligned}
& a=0, \\
& c=\frac{k-b^{\prime \prime}-b^{\prime}}{2},
\end{aligned}
$$


where $k$ is constant. The last equation of system (22) can now be written as

$$
\left(c p_{0}+d\right) \varphi^{\prime}-\left(c-2 b^{\prime}\right) \varphi=\left(c^{\prime \prime}-c^{\prime}\right) p_{0}+d^{\prime \prime}-d^{\prime}
$$

The equation obtained is a first-order linear differential equation with respect to the function $\varphi=\varphi\left(p_{0}\right)$. Here the variable $t$ can be considered as a parameter. Under the condition that $c \neq 0, c-2 b^{\prime} \neq$, and $b^{\prime} \neq 0$, the general solution of equation (23) has the form:

$$
\varphi\left(p_{0}\right)=R(t)\left(p_{0}+\frac{d}{c}\right)^{1-2\left(b^{\prime} / c\right)}+\frac{c^{\prime \prime}-c^{\prime}}{2 b^{\prime}}\left(p_{0}+\frac{d}{c}\right)+\frac{d\left(c^{\prime \prime}-c^{\prime}\right)-c\left(d^{\prime \prime}-d^{\prime}\right)}{c\left(c-2 b^{\prime}\right)}
$$

where $R(t)$ is an arbitrary function.

We must pick out those of functions of form (24) that are nonlinear with respect to $p_{0}$ and are independent of $t$. A function of form (24) satisfies these conditions if and only if

$$
\begin{aligned}
& R=\alpha=\text { const }, \\
& \frac{d}{c}=\beta=\text { const }, \\
& 1-2 \frac{b^{\prime}}{c}=\gamma=\text { const, } \\
& \frac{c^{\prime \prime}-c^{\prime}}{2 b^{\prime}}=\text { const, } \\
& \frac{d\left(c^{\prime \prime}-c^{\prime}\right)-c\left(d^{\prime \prime}-d^{\prime}\right)}{c\left(c-2 b^{\prime}\right)}=\text { const. }
\end{aligned}
$$

From these relations we obtain

$$
c=-\frac{\alpha}{2}(1+k) \mathrm{e}^{k t}, \quad d=\beta c, \quad b^{\prime}=\frac{1-\gamma}{2} c,
$$

where $k=\frac{1-\gamma}{3+\gamma}$. Eventually,

$$
\varphi\left(p_{0}\right)=\alpha\left(p_{0}+\beta\right)^{\gamma}-\frac{2 \gamma+2}{(\gamma+3)^{2}}
$$

It remains to consider the cases excluded from the previous considerations. If $c=0$ and $b^{\prime}=0$, then $\varphi$ is a linear function. If $c=0$, then the solution of equation (5) is nonlinear:

$$
\varphi\left(p_{0}\right)=\alpha \mathrm{e}^{\beta p_{0}}-\frac{2}{\beta}
$$

Thus, the following result is true: 
Theorem. Among all second-order nonlinear differential equations of the form

$$
y^{\prime \prime}=y^{\prime}+\varphi(y)
$$

the following ones have a two-dimensional Lie algebra of point symmetries:

i) $y^{\prime \prime}=y^{\prime}+\alpha(y+\beta)^{\gamma}-\frac{2 \gamma+2}{(\gamma+3)^{2}}, \quad \alpha, \beta, \gamma \in \mathbb{R}, \gamma \neq 1,-3$

ii) $y^{\prime \prime}=y^{\prime}+\alpha \mathrm{e}^{\beta y}-\frac{2}{\beta}, \quad \alpha, \beta \in \mathbb{R}, \beta \neq 0$.

In addition, the following functions can be chosen as a basis of the space of generating functions for symmetries of these equations:

i) $f_{1}=p_{1}, f_{2}=\mathrm{e}^{k t}\left(p_{1}-\frac{k+1}{2} p_{0}\right), k=\frac{1-\gamma}{3+\gamma}$;

ii) $f_{1}=p_{1}, f_{2}=\mathrm{e}^{-t}\left(p_{1}-\frac{2}{\beta}\right)$.

4.6. Let $\mathcal{E} \subset J^{k}(\mathbb{R}, M)$ be a system of differential equations on a manifold $M$, $\operatorname{dim} M \geqslant 2$. Assume that $\mathcal{E}$ is solvable with respect to derivatives of the highest order. Then $\mathcal{E}$ can be identified with a vector field $\mathcal{D}$ on $J^{k-1}(\mathbb{R}, M)$ lying in the Cartan distribution. The projection of $\mathcal{D}$ on $\mathbb{R}$ is the field $\frac{\partial}{\partial t}$. Therefore, $\mathcal{D}$ can be written as

$$
\mathcal{D}=\frac{\partial}{\partial t}+\mathcal{D}_{0}
$$

where $\mathcal{D}_{0}$ is a $\pi_{k-1}$-vertical vector field, i.e. a vector field tangent to fibres of the projection $\pi_{k-1}: J^{k}(\mathbb{R}, M) \rightarrow \mathbb{R}$.

The field $\mathcal{D}$ generates the characteristic distribution for $C(\mathcal{E})$. Therefore, shuffling infinitesimal symmetries of the system $\mathcal{E}$ can be identified with $\pi_{k-1}$-vertical vector fields $X$ on $J^{k-1}(\mathbb{R}, M)$.

The condition that a vector field $X$ be a symmetry is equivalent to the condition that $\mathcal{D}$ and $[\mathcal{D}, X]$ be proportional. Comparing the $\frac{\partial}{\partial t}$-components of these two vector fields, we obtain the following result:

Proposition. Shuffling symmetries of the system $\mathcal{E}$ are $\pi_{k-1}$-vertical vector fields on $J^{k-1}(\mathbb{R}, M)$ satisfying the Lie equation

$$
\dot{X}=\left[X, \mathcal{D}_{0}\right]
$$

where $\dot{X}=\left[\frac{\partial}{\partial t}, X\right]$.

Proof. It is sufficient to note that

$$
[\mathcal{D}, X]=\left[\frac{\partial}{\partial t}+\mathcal{D}_{0}, X\right]=\dot{X}+\left[\mathcal{D}_{0}, X\right]
$$

4.7. Let us use the results of the previous section in order to describe (in local coordinates) shuffling symmetries of the following system of differential equations:

$$
\left\{\begin{array}{l}
p_{k}^{1}=F^{1}\left(t, \ldots, p_{j}^{i}, \ldots\right) \\
\vdots \\
p_{k}^{n}=F^{n}\left(t, \ldots, p_{j}^{i}, \ldots\right)
\end{array}\right.
$$


where $i=1, \ldots, n, j=0,1, \ldots, k-1$.

The vector field $\mathcal{D}$ can be written as

$$
\mathcal{D}=\frac{\partial}{\partial t}+\sum_{i, j} p_{j+1}^{i} \frac{\partial}{\partial p_{j}^{i}}+\sum_{i} F^{i} \frac{\partial}{\partial p_{k-1}^{i}}
$$

Let

$$
X=\sum_{i, j} X_{j}^{i} \frac{\partial}{\partial p_{j}^{i}}
$$

be a shuffling symmetry of system (29), where

$$
X_{j}^{i}=X_{j}^{i}\left(t, \ldots, p_{l}^{r}, \ldots\right), \quad r=1, \ldots, n, l=0,1, \ldots, k-1 .
$$

Then

$$
\dot{X}=\sum_{i, j} \frac{\partial X_{j}}{\partial t} \frac{\partial}{\partial p_{j}^{i}}
$$

and

$$
\left[\mathcal{D}_{0}, X\right]=\sum_{i, j}\left(\mathcal{D}_{0}\left(X_{j}^{i}\right)-X_{j+1}^{i}\right) \frac{\partial}{\partial p_{j}^{i}}+\sum_{i}\left(\mathcal{D}_{0}\left(X_{k-1}^{i}\right)-\sum_{r, l} X_{l}^{r} \frac{\partial F^{i}}{\partial p_{l}^{r}}\right) \frac{\partial}{\partial p_{k-1}^{i}}
$$

Therefore, the vector field $X$ is a shuffling symmetry of system (29) if and only if

$$
\left\{\begin{array}{l}
\mathcal{D}\left(X_{j}^{i}\right)=X_{j+1}^{i}, \quad i=1, \ldots, n, j=0, \ldots, k-2 \\
\mathcal{D}\left(X_{k-1}^{i}=\sum_{r, l} X_{l}^{r} \frac{\partial F^{i}}{\partial p_{l}^{r}}, \quad i=1, \ldots, n\right.
\end{array}\right.
$$

Put $f^{i}=X_{0}^{i}$. Then from the first equations of system (32) it follows that

$$
X_{0}^{i}=f^{i}, X_{1}^{i}=\mathcal{D}\left(f^{i}\right), \ldots, X_{j}^{i}=\mathcal{D}^{j}\left(f^{i}\right), \ldots, X_{k-1}^{i}=\mathcal{D}^{k-1}\left(f^{i}\right) .
$$

Thus every shuffling symmetry can be written as

$$
X_{f}=\sum_{i, j} \mathcal{D}^{j}\left(f^{i}\right) \frac{\partial}{\partial t}
$$

for some vector-valued function

$$
\begin{gathered}
f=\left(f^{1}, \ldots, f^{n}\right), \\
f^{i}=f^{i}\left(t, \ldots, p_{l}^{r}, \ldots\right), \quad r=1, \ldots, n, j=0,1, \ldots, k-1 .
\end{gathered}
$$

The function $f$ is called the generating function of the symmetry $X_{f}$. 
The rest of the equations of system (32) reduce to the Lie equations for $f$ :

$$
\mathcal{D}^{k}\left(f^{i}\right)-\sum_{r, l} \frac{\partial F^{i}}{\partial p_{l}^{r}} \mathcal{D}^{l}\left(f^{r}\right)=0, \quad i=1, \ldots, n
$$

By $L_{F}$ denote the matrix differential operator (the Lie operator) $L_{F}=\left\|L_{i r}\right\|$, where

$$
L_{i r}=\delta_{i r} \mathcal{D}^{k}-\sum_{l=0}^{k-1} \frac{\partial F^{i}}{\partial p_{l}^{r}} \mathcal{D}^{l}
$$

Then Lie equations (34) can be written in matrix form:

$$
L_{F}\left(f^{t}\right)=0
$$

where $f^{t}$ is the transpose of the row vector $f$.

The symmetries corresponding to solutions of system (36) form a Lie algebra with respect to commutation of vector fields. This operation determines Jacobi bracket on the space of generating functions: if $f$ and $g$ are generating functions, then their Jacobi bracket is the vector-valued function $[f, g]$ such that its $i$-th component $[f, g]^{i}$ has the form:

$$
[f, g]^{i}=\sum_{r, l}\left(\mathcal{D}^{l}\left(f^{r}\right) \frac{\partial g^{i}}{\partial p_{l}^{r}}-\mathcal{D}^{l}\left(g^{r}\right) \frac{\partial f^{i}}{\partial p_{l}^{r}}\right) .
$$

Thus, the following result is true:

Theorem. If $\mathcal{E}$ is a system of differential equations resolved with respect to derivatives of the highest order, then its shuffling symmetries can be represented as (33), generating functions $f$ satisfying Lie system (34). The solution set of this system is a Lie algebra with respect to Jacobi bracket (37).

\section{§5. LiNEAR SYMMETRIES}

In this section we shall consider linear differential equations with respect to functions $y: \mathbb{R} \rightarrow \mathbb{R}$

$$
L_{y}=y^{(k)}+a_{1}(t) y^{(k-1)}+\cdots+a_{k}(t) y=0
$$

and their linear symmetries, i.e. symmetries preserving linear structure of the solution space.

5.1. Consider first the differential equation $\mathcal{E} \subset J^{k}(\mathbb{R}, \mathbb{R})$ given by

$$
p_{k}=F\left(t, p_{0}, \ldots, p_{k-1}\right)
$$

Let us find a condition for a function $f\left(t, p_{0}, \ldots, p_{k-1}\right)$ to be a generating function of a shuffling symmetry of $\mathcal{E}$. 
Let $\left\{\varphi_{s}\right\}$ be the one-parameter group of translations along the vector field $X_{f}$ and let $h=h(t)$ be a solution of $(1)$. Then

$$
\varphi_{s}\left(j_{k}(h)\right) \subset \mathcal{E}
$$

Let $\varphi_{s}\left(j_{k}(h)\right)=j_{k}\left(h_{s}\right) ;$ then

$$
\begin{aligned}
h_{s} & =h+s f_{h}+o(s), \\
h_{s}^{\prime} & =h^{\prime}+s(\mathcal{D} f)_{h}+o(s), \\
\vdots & \\
h_{s}^{(k-1)} & =h^{(k-1)}+s\left(\mathcal{D}^{k-1} f\right)_{h}+o(s),
\end{aligned}
$$

where for a function $G=G\left(t, p_{0}, \ldots, p_{k-1}\right)$ by $G_{h}$ we denote its restriction to the curve $j_{k-1}(h): G_{h}(t)=G\left(t, h(t), \ldots, h^{(k-1)}(t)\right)$.

Thus, a function $f=f\left(t, p_{0}, \ldots, p_{k-1}\right)$ is a generating function of a shuffling symmetry if the one-parameter family of functions

$$
h_{s}=h+s f_{n}
$$

corresponding to an arbitrary solution $h(t)$ satisfies equation (1) up to $o(s)$ :

$$
h_{s}^{(k)}-F\left(t, h_{s}, \ldots, h_{s}^{(k-1)}\right)=o(s) .
$$

5.2. We shall use observations of the previous item in order to describe linear symmetries of linear differential equations.

Consider the linear differential equation

$$
L y=0
$$

given by the operator

$$
L=\partial^{k}+a_{1}(t) \partial^{k-1}+\cdots+a_{k}(t)
$$

where $\partial=\frac{\partial}{\partial t}$.

Definition. Shuffling symmetries of differential equation (4) are called its linear symmetries if their generating functions $f$ are linear:

$$
f=b_{1}(t) p_{k-1}+\cdots+b_{k}(t) p_{0} .
$$

To a function $f$ of form (6) we associate the operator $\Delta\left(=\Delta_{f}\right)$ given by

$$
\Delta=b_{1}(t) \partial^{k-1}+\cdots+b_{k}(t)
$$

Let us write the Lie equation for a generating function $f$ in terms of the corresponding operator $\Delta$, using relation (3). But first prove the following result: 
Lemma 1. For an arbitrary differential operator

$$
M=m_{0}(t) \partial^{n}+m_{1}(t) \partial^{n-1}+\cdots+m_{n}(t)
$$

of order $n \geqslant k$ there exist differential operators $N$ and $R$ of order $n-k$ and $k-1$ respectively such that

$$
M=N \circ L+R \text {. }
$$

In addition, the operators $N$ and $R$ are uniquely defined.

Proof. We shall prove this by induction on the order $n$ of $M$. If $n \leqslant k-1$, then it is obvious that the operators $N$ and $R$ exist. Assume that these operators exist for all operators of order $\leqslant n-1$. For an operator $M$ of order $n$ we have

$$
M=\left(m_{0}(t) \partial^{n-k}\right) \circ L+M_{1}
$$

where the order of $M_{1}$ is not greater than $n-1$. Then $M_{1}=N_{1} \circ L+R_{1}$ and

$$
M=\left(m_{0}(t) \partial^{n-k}+N_{1}\right) \circ L+R_{1} .
$$

Let us prove that $N$ and $R$ are unique. Suppose we are given two representations:

$$
M=N_{1} \circ L+R_{1} \text { and } M=N_{2} \circ L+R_{2},
$$

where $N_{1} \neq N_{2}$ and $R_{1} \neq R_{2}$. Then $\left(N_{1}-N_{2}\right) \circ L=R_{2}-R_{1}$. But the order of the operator $\left(N_{1}-N_{2}\right) \circ L$ is not less than $k$, whereas the order of $R_{2}-R_{1}$ is not greater than $k-1$. Therefore, $N_{1}-N_{2}=0$ and $R_{2}-R_{1}=0$.

Remark. It can be proved similarly that $M$ can be written uniquely as

$$
M=L \circ N+R
$$

Lemma 2. Let $M$ be a differential operator such that

$$
M(h)=0
$$

for every solution $h=h(t)$ of the equation $L(h)=0$. Then there exists a differential operator $N$ such that $M=N \circ L$.

Proof. Using Lemma 1, write the operator $M$ as $M=N \circ L+R$. Then $M(h)=R(h)$ for all solutions $h(t)$ and

$$
R=r_{1}(t) \partial^{k-1}+\cdots+r_{k-1}(t)
$$

Every solution $h(t)$ can be uniquely determined by the set

$$
h_{0}=h\left(t_{0}\right), h_{1}=h^{\prime}\left(t_{0}\right), \ldots, h_{k-1}=h^{(k-1)}\left(t_{0}\right),
$$


where $t_{0}$ is an arbitrary point of $\mathbb{R}$. Since

$$
R(h)\left(t_{0}\right)=r_{1}\left(t_{0}\right) h_{k-1}+\cdots+r_{k-1}\left(t_{0}\right) h_{0}=0
$$

for all sets $\left(t_{0}, h_{0}, \ldots, h_{k-1}\right)$, we obtain $r_{1} \equiv 0, \ldots, r_{k-1} \equiv 0$.

5.3. Theorem. A differential operator $\Delta$ corresponds some shuffling symmetry if and only if there exists a differential operator $\bar{\Delta}$ such that

$$
L \circ \Delta=\bar{\Delta} \circ L
$$

Remarks.

(1) The order of $\bar{\Delta}$, as well as that of $\Delta$, does not exceed $k-1$.

(2) The operator $\bar{\Delta}$ is uniquely defined.

Proof of the Theorem. Let $\Delta$ be an operator corresponding to some shuffling symmetry. Then

$$
L(h+s \Delta(h))=s(L \circ \Delta)(h)=o(s)
$$

for all solutions $h$ of the equation $L(h)=0$. Therefore, $(L \circ \Delta)(h)=0$ for all solutions $h$. Then from lemma 2 it follows that there exists an operator $\bar{\Delta}$ such that $L \circ \Delta=\bar{\Delta} \circ L$. It is obvious that the converse is also true.

\subsection{Definition.}

(1) Let $M$ be an operator of the form:

$$
M=m_{0}(t) \partial^{n}+\cdots+m_{n-1}(t) \partial+m_{n}(t)
$$

The operator

$$
M^{t}=(-1)^{n} \partial^{n} \circ m_{0}+\cdots+(-1) \partial \circ m_{n-1}+m_{n},
$$

operating as follows

$$
M^{t}(h)=(-1)^{n}\left(m_{0} h\right)^{(n)}+\cdots+(-1)\left(m_{n-1} h\right)^{\prime}+m_{n} h,
$$

is called (formally) adjoint to $M$.

(2) A differential operator $M$ of form (12) is called self-adjoint if $M^{t}=M$ and anti-self-adjoint if $M^{t}=-M$.

It is easy to verify that a self-adjoint operator always has even order $n=2 r$ and can be written as

$$
M(h)=\sum_{j=0}^{r}\left(f_{j}(t) h^{(j)}\right)^{(j)}
$$

Correspondingly, an anti-self-adjoint operator always has odd order $n=2 r+1$ and can be written as

$$
M(h)=\sum_{j=0}^{r}\left[\left(f_{j}(t) h^{(j)}\right)^{(j+1)}+\left(f_{j}(t) h^{(j+1)}\right)^{(j)}\right] .
$$


Theorem.[J.Lagrange]. Let $M$ be an arbitrary differential operator of form (12) and $u(t), v(t)$ two functions. Then

$$
v M(u)-u M^{t}(v)=\partial P_{M}(u, v)
$$

where

$$
P_{M}(u, v)=\sum_{k=1}^{n} \sum_{j=0}^{k-1}(-1)^{j}\left(\partial^{k-j-1} u, \partial^{j}\left(m_{n-k} v\right)\right)
$$

5.5. Let $V$ be a finite-dimensional vector space and $A_{j}: \mathbb{R} \rightarrow \operatorname{Hom}(V, W)$ functions defined on $\mathbb{R}$.

Consider the differential operator

$$
M=A_{0}(t) \partial^{n}+\cdots+A_{n}(t)
$$

operating on vector-valued functions $u: \mathbb{R} \rightarrow V$. The operator

$$
M^{t}=(-1)^{n} \partial^{n} \circ A_{0}^{t}+\cdots+A_{n}^{t},
$$

operating on vector-valued functions $w: \mathbb{R} \rightarrow W^{*}$, is called adjoint to $M$. Here by $A^{t}$ we denote the operator adjoint to $A$ in sense of linear algebra:

$$
\langle A u, w\rangle=\left\langle u, A^{t} w\right\rangle
$$

for all $u \in V, w \in W^{*}$, where $<,>$ is the natural pairing of the spaces $W$ and $W^{*}$.

Theorem.[J.Lagrange]. For an arbitrary operator $M$ and any two vector-valued functions $u: \mathbb{R} \rightarrow V, v: \mathbb{R} \rightarrow W^{*}$ the following relation holds:

$$
\langle M u, v\rangle-\left\langle u, M^{t} v\right\rangle=\partial P_{M}(u, v)
$$

where

$$
P_{M}(u, v)=\sum_{k=1}^{n} \sum_{j=0}^{k-1}(-1)^{j}\left\langle\partial^{k-j-1} u, \partial^{j}\left(A_{n-k}^{t} v\right)\right\rangle .
$$

Proof. It is easy to check that

$$
\left\langle B \partial^{k} u, v\right\rangle-\left\langle u,(-1)^{k} \partial^{k} B v\right\rangle=\partial \sum_{j=0}^{k-1}(-1)^{j}\left\langle\partial^{k-j-1} u, \partial^{j} B^{t} v\right\rangle .
$$

It remains to apply this formula to every term of the operator $M$.

\subsection{Examples.}


(1) Every anti-self-adjoint operator of order 1 has the form:

$$
M(h)=(f(t) h)^{\prime}+f(t) h^{\prime}=2 f(t) h^{\prime}+f^{\prime}(t) h
$$

or

$$
M=2 f(t) \partial+f^{\prime}(t)
$$

(2) Every self-adjoint operator of order 2 has the form:

$$
M(h)=\left(f_{1}(t) h^{\prime}\right)^{\prime}+f_{0}(t) h=f_{1}(t) h^{(2)}+f_{1}^{\prime}(t) h^{\prime}+f_{0}(t) h,
$$

or

$$
M=f_{1}(t) \partial^{2}+f_{1}^{\prime}(t) \partial+f_{0}(t)
$$

(3) Anti-self-adjoint operators of order 3 have the form:

$$
\begin{aligned}
M(h)=\left(f_{1}(t) h^{(2)}\right)^{\prime}+\left(f_{1}(t) h^{\prime}\right)^{(2)}+f_{0}(t) h^{\prime}+\left(f_{0}(t) h\right)^{\prime} & = \\
& =2 f_{1} h^{(3)}+3 f_{1}^{\prime} h^{(2)}+\left(f_{1}^{\prime \prime}+2 f_{0}\right) h^{\prime}+f_{0}^{\prime} h
\end{aligned}
$$

or

$$
M=2 f_{1} \partial^{3}+3 f_{1}^{\prime} h^{2}+\left(f_{1}^{\prime \prime}+2 f_{0}\right) \partial+f_{0}^{\prime} .
$$

The corresponding Lagrange formula have the form:

$\left(1^{\prime}\right) u M(v)+v M(u)=\partial P_{M}(u, v)$, where $P_{M}(u, v)=2 f u v$;

$\left(2^{\prime}\right) u M(v)-v M(u)=\partial P_{M}(u, v)$, where $P_{M}(u, v)=f_{1}\left(u^{\prime} v-u v^{\prime}\right)$;

$\left(3^{\prime}\right) u M(v)+v M(u)=\partial P_{M}(u, v)$, where $P_{M}(u, v)=2 f_{1}\left(u^{\prime \prime} u-u^{\prime} v^{\prime}+u v^{\prime \prime}\right)+$ $f_{1}^{\prime}\left(u^{\prime} v+u v^{\prime}\right)+2 f_{0} u v$.

(4) If $M=A_{0}(t) \partial+A_{1}(t)$ is a matrix differential operator, then $M^{t}=-\partial A_{0}^{t}+$ $A_{1}^{t}-A_{0}^{t} \partial-\left(A_{0}^{t}\right)^{\prime}+A_{1}^{t}$. Therefore, $M$ is self-adjoint if $W=V^{*}$ and

$$
A_{0}^{t}=-A_{0},-A_{1}^{t}+A_{1}=A_{0}^{\prime}
$$

so that $A_{1}=1 / 2 A_{0}^{\prime}+B_{1}$, where $B_{1}$ is symmetric, i.e. $B_{1}=B_{1}^{t}$. Thus, the formula

$$
M=A_{0} \partial+1 / 2 A_{0}^{\prime}+C_{1}
$$

where $A_{0}$ is skew-symmetric and $C_{1}$ is symmetric, gives a complete description of self-adjoint operators of order 1 . The corresponding Lagrange formula has the form:

$$
\langle M u, v\rangle-\langle u, M v\rangle=\partial P_{M}(u, v)
$$

where $P_{M}(u, v)=\left\langle u, A_{0}^{t} v\right\rangle$.

If $M$ is anti-self-adjoint, then $A_{0}^{t}=A_{0}$ and $\left(A_{0}^{t}\right)^{\prime}-A_{1}^{t}=A_{1}$. Thus, the formula

$$
M=A_{0} \partial+1 / 2 A_{0}^{\prime}+C_{1}
$$


where $A_{0}$ is symmetric and $C_{1}$ is skew-symmetric, gives a complete description of anti-self-adjoint operators of order 1 . The corresponding Lagrange formula has the form:

$$
\langle M u, v\rangle+\langle u, M v\rangle=\partial P_{M}(u, v),
$$

where $P_{M}(u, v)=\left\langle u, A_{0}^{t} v\right\rangle$.

5.7. By $\Pi=\Pi(L)$ denote the set of pairs $(\Delta, \bar{\Delta})$ of differential operators of order $\leqslant k-1$ such that $\Delta$ and $\bar{\Delta}$ satisfy conditions of theorem 5.3. Note that $\Pi(L)$ is a vector space over $\mathbb{R}$, and if $(\Delta, \bar{\Delta}) \in \Pi(L)$, then $\left(\bar{\Delta}^{t}, \Delta^{t}\right) \in \Pi\left(L^{t}\right)$.

In particular, if $L$ is self-adjoint or anti-self-adjoint, then the operation of adjoining operators generates the involution

$$
(\Delta, \bar{\Delta}) \mapsto\left(\bar{\Delta}^{t}, \Delta^{t}\right)=(\Delta, \bar{\Delta})^{t}
$$

of the space $\Pi(L)$. Thus, if an operator $\Delta$ corresponds to some shuffling symmetry of the equation $L h=0$, then so does $\bar{\Delta}^{t}$. Using the involution just defined, we write $\Pi$ as a direct sum:

$$
\Pi(L)=\Pi_{0}(L) \oplus \Pi_{1}(L)
$$

where

$$
\begin{aligned}
& \Pi_{0}(L)=\left\{(\Delta, \bar{\Delta}) \in \Pi(L) \mid(\Delta, \bar{\Delta})^{t}=-(\Delta, \bar{\Delta})\right\}, \\
& \Pi_{1}(L)=\left\{(\Delta, \bar{\Delta}) \in \Pi(L) \mid(\Delta, \bar{\Delta})^{t}=-(\Delta, \bar{\Delta})\right\} ;
\end{aligned}
$$

in other words,

$$
\begin{aligned}
& \Pi_{0}(L)=\left\{\left(\Delta,-\Delta^{t}\right) \in \Pi(L)\right\}, \\
& \Pi_{1}(L)=\left\{\left(\Delta, \Delta^{t}\right) \in \Pi(L)\right\} .
\end{aligned}
$$

Theorem. Let $L$ be a self-adjoint or anti-self-adjoint operator. Then the set of operators corresponding to linear shuffling symmetries of the equation $L h=0$ can be written as the direct sum of the solution spaces of the following equations:

$$
\begin{aligned}
& \left(\Pi_{0}\right): L \circ \Delta=-\Delta^{t} \circ L, \\
& \left(\Pi_{1}\right): L \circ \Delta=\Delta^{t} \circ L .
\end{aligned}
$$

5.8. Let us indicate some algebraic constructions related to the questions under consideration. Omitting the condition that operators $\Delta, \bar{\Delta}$ have order $\leqslant k-1$, we obtain a new space of pairs of operators. We denote it by $\widehat{\Pi}(L)$. The space $\widehat{\Pi}(L)$ can be supplied with some additional algebraic structures. Let $\left(\Delta_{1}, \bar{\Delta}_{1}\right) \in \widehat{\Pi}(L)$ and $\left(\Delta_{2}, \bar{\Delta}_{2}\right) \in \widehat{\Pi}(L)$. Since

$$
L \circ \Delta_{1} \circ \Delta_{2}=\bar{\Delta}_{1} \circ L \circ \Delta_{2}=\bar{\Delta}_{1} \circ \bar{\Delta}_{2} \circ L
$$

we have $\left(\Delta_{1} \circ \Delta_{2}, \bar{\Delta}_{1} \circ \bar{\Delta}_{2}\right) \in \widehat{\Pi}(L)$. Thus, we obtain multiplication in $\widehat{\Pi}(L)$, and this operation turns $\widehat{\Pi}(L)$ into an associative algebra. 
If $L$ is self-adjoint or anti-self-adjoint, the space $\widehat{\Pi}(L)$ is closed with respect to involution (28) and, as before,

$$
\widehat{\Pi}(L)=\widehat{\Pi}_{0}(L)+\widehat{\Pi}_{1}(L) .
$$

In addition, $\widehat{\Pi}_{1}(L)$ is closed with respect to anticommutator:

$$
\left\{\left(\Delta_{1}, \bar{\Delta}_{1}\right),\left(\Delta_{2}, \bar{\Delta}_{2}\right)\right\}=\left(\frac{\Delta_{1} \circ \Delta_{2}+\Delta_{2} \circ \Delta_{1}}{2}, \frac{\bar{\Delta}_{1} \circ \bar{\Delta}_{2}+\bar{\Delta}_{2} \circ \bar{\Delta}_{1}}{2}\right)
$$

whereas $\widehat{\Pi}_{0}(L)$ is closed with respect to commutator:

$$
\left[\left(\Delta_{1}, \bar{\Delta}_{1}\right),\left(\Delta_{2}, \bar{\Delta}_{2}\right)\right]=\left(\Delta_{1} \circ \Delta_{2}-\Delta_{2} \circ \Delta_{1}, \bar{\Delta}_{1} \circ \bar{\Delta}_{2}-\bar{\Delta}_{2} \circ \bar{\Delta}_{1}\right) .
$$

Let $\left(\Delta_{1}, \bar{\Delta}_{1}\right) \in \widehat{\Pi}_{0}(L)$ and $\left(\Delta_{2}, \bar{\Delta}_{2}\right) \in \widehat{\Pi}_{1}(L)$. Then formula (33) gives an action of a Lie algebra on a vector space. Thus, $\widehat{\Pi}_{0}(L)$ is a Lie algebra, $\widehat{\Pi}_{1}(L)$ is a Jordan algebra, and the Lie algebra $\widehat{\Pi}_{0}(L)$ acts on $\widehat{\Pi}_{1}(L)$.

Now let $(\Delta, \bar{\Delta}) \in \widehat{\Pi}(L)$ be an arbitrary pair. The operators $\Delta$ and $\bar{\Delta}$ can be written as

$$
\begin{aligned}
& \Delta=N \circ L+R(\Delta), \\
& \bar{\Delta}=L \circ M+S(\bar{\Delta}),
\end{aligned}
$$

where the orders or $R(\Delta)$ and $S(\bar{\Delta})$ do not exceed $k-1$. Since $L \circ \Delta=\bar{\Delta} \circ L$, we obtain

$$
L \circ(N-M) \circ L=S(\bar{\Delta}) \circ L-L \circ R(\Delta) .
$$

If $N \neq M$, then the order of the operator standing in the left-hand side of the equality must be greater than $2 k$. Therefore, $M=N$ and

$$
(R(\Delta), S(\bar{\Delta})) \in \Pi(L) .
$$

Thus, the mapping

$$
\widehat{\Pi}(L) \ni(\Delta, \bar{\Delta}) \mapsto(R(\Delta), S(\bar{\Delta})) \in \Pi(L),
$$

which is obviously an epimorphism, establishes a connection between $\widehat{\Pi}(L)$ and the space of shuffling symmetries.

Example.[J.Dixmier]. The differential operator

$$
\Delta=\partial^{4}+\left(2 t^{3}+2 \alpha\right) \partial^{2}+6 t^{2} \partial+\left(t^{6}+2 \alpha t^{3}+8 t+\alpha^{2}\right)
$$

commutes with the operator

$$
\begin{aligned}
L=\partial^{6}+3\left(t^{3}+\alpha\right) \partial^{4}+18 t^{2} \partial^{3}+\left(3 t^{6}+6 \alpha t^{3}+45 t+3 \alpha^{2}\right) \partial^{2}+ \\
+\left(18 t^{5}+18 \alpha t^{2}+27\right) \partial+\left(t^{9}+3 \alpha t^{6}+33 t^{4}+3 \alpha^{2} t^{3}+21 \alpha t+\alpha^{3}\right)
\end{aligned}
$$


where $\alpha \in \mathbb{R}$ is some constant number.

Thus, the equation $L h=0$ has a shuffling symmetry of order 4 . Its generating function has the form:

$$
f=p_{4}+\left(2 t^{3}+2 \alpha\right) p_{2}+6 t^{2} p_{1}+\left(t^{6}+2 \alpha t^{3}+8 t+\alpha^{2}\right) p_{0}
$$

5.9. Operators of the form

$$
L=\partial^{2}+f
$$

are called Hill operators. The function $f=f(t)$ is called the potential.

Let us find linear shuffling symmetries of the equation $L h=0$. We shall first describe the space $\Pi(L)$, using the fact that $L$ is self-adjoint.

Let $\Delta=x+y \partial ;$ then $\Delta^{t}=\left(x-y^{\prime}\right)-y \partial$ and

$$
\begin{aligned}
& L \circ \Delta=\left(\partial^{2}+f\right)(x+y \partial)=y \partial^{3}+\left(x+2 y^{\prime}\right) \partial^{2}+\left(y^{\prime \prime}+2 x^{\prime}+y f\right) \partial+x^{\prime \prime}+f x, \\
& \Delta^{t} \circ L=\left(x-y^{\prime}-y \partial\right)\left(\partial^{2}+f\right)=-y \partial^{3}+\left(x-y^{\prime}\right) \partial^{2}-y f \partial-y f^{\prime}-y^{\prime} f+f x .
\end{aligned}
$$

Therefore, if $\Delta \in \Pi_{0}(L)$, then $L \circ \Delta+\Delta^{t} \circ L=0$ so that

$$
\left\{\begin{array}{l}
2 x+y^{\prime}=0 \\
2 x^{\prime}+y^{\prime \prime}=0 \\
x^{\prime \prime}+2 x f-y f^{\prime}-y^{\prime} f=0
\end{array}\right.
$$

From the first equation we obtain

$$
x=-1 / 2 y^{\prime}
$$

Substituting this into the last equation, we obtain

$$
y^{\prime \prime \prime}+4 f y^{\prime}+2 f^{\prime} y=0
$$

If $\Delta \in \Pi_{1}(L)$, then $L \circ \Delta-\Delta^{t} \circ L=0$, so that $y=0$ and $x^{\prime}=0$. Thus, $\Delta \in \Pi_{1}(L)$ if and only if $\Delta(h)=c h$ for some constant number $c \in \mathbb{R}$.

Consider equation (35). The corresponding operator

$$
M=\partial^{3}+4 f \partial+2 f^{\prime}
$$

is anti-self-adjoint. The solution space of the equation is 3-dimensional. As a solution space of a Lie equation, this space is a Lie algebra with respect to bracket

$$
\left[y_{1}, y_{2}\right]=y_{1} y_{2}^{\prime}-y_{1}^{\prime} y_{2}
$$

reduced from Jacobi bracket. 
Let $\left\{Y_{1}, Y_{2}, Y_{3}\right\}$ be a fundamental system of solutions of equation (35); in other words, at some point $t_{0} \in \mathbb{R}$, the solutions $Y_{1}, Y_{2}$, and $Y_{3}$ satisfy the following initial conditions:

$$
\left\{\begin{array} { l } 
{ Y _ { 1 } ( t _ { 0 } ) = 1 , } \\
{ Y _ { 1 } ^ { \prime } ( t _ { 0 } ) = 0 , } \\
{ Y _ { 1 } ^ { \prime \prime } ( t _ { 0 } ) = 0 ; }
\end{array} \quad \left\{\begin{array} { l } 
{ Y _ { 2 } ( t _ { 0 } ) = 0 , } \\
{ Y _ { 2 } ^ { \prime } ( t _ { 0 } ) = 1 , } \\
{ Y _ { 2 } ^ { \prime \prime } ( t _ { 0 } ) = 0 ; }
\end{array} \quad \left\{\begin{array}{l}
Y_{3}\left(t_{0}\right)=0 \\
Y_{3}^{\prime}\left(t_{0}\right)=0 \\
Y_{3}^{\prime \prime}\left(t_{0}\right)=1
\end{array}\right.\right.\right.
$$

Commutators of these solutions are also solutions and from (35) and (38) it follows that

$$
\begin{aligned}
& {\left[Y_{1}, Y_{2}\right]\left(t_{0}\right)=1,\left[Y_{1}, Y_{2}\right]^{\prime}\left(t_{0}\right)=0,\left[Y_{1}, Y_{2}\right]^{\prime \prime}\left(t_{0}\right)=-4 f\left(t_{0}\right),} \\
& {\left[Y_{1}, Y_{3}\right]\left(t_{0}\right)=0,\left[Y_{1}, Y_{3}\right]^{\prime}\left(t_{0}\right)=1,\left[Y_{1}, Y_{3}\right]^{\prime \prime}\left(t_{0}\right)=0} \\
& {\left[Y_{2}, Y_{3}\right]\left(t_{0}\right)=0,\left[Y_{2}, Y_{3}\right]^{\prime}\left(t_{0}\right)=0,\left[Y_{2}, Y_{3}\right]^{\prime \prime}\left(t_{0}\right)=1 .}
\end{aligned}
$$

Therefore, for all $t \in \mathbb{R}$ we have

$$
\left\{\begin{array}{l}
{\left[Y_{1}, Y_{2}\right]\left(t_{0}\right)=-Y_{1}-4 f\left(t_{0}\right) Y_{3}} \\
{\left[Y_{1}, Y_{3}\right]\left(t_{0}\right)=-Y_{2}} \\
{\left[Y_{2}, Y_{3}\right]\left(t_{0}\right)=-Y_{3}}
\end{array}\right.
$$

Thus, the solution space of equation $(35)$ is isomorphic to the Lie algebra $\mathfrak{s l}(2, \mathbb{R})$.

If $f=0$, then equation (35) takes the form:

$$
y^{\prime \prime \prime}=0
$$

The corresponding realization of $\mathfrak{s l}(2, \mathbb{R})$ in terms of vector fields on $\mathbb{R}$ has the form:

$$
\frac{\partial}{\partial t}, t \frac{\partial}{\partial t}, \frac{1}{2} t^{2} \frac{\partial}{\partial t}
$$

Note that a shuffling symmetry

$$
y=\lambda_{1} t^{2}+\lambda_{2} t+\lambda_{3}
$$

of the Hill equation $L h=0$ belongs to $\mathfrak{s l}(2, \mathbb{R})$ if and only if the potential $f(t)$ satisfies the condition

$$
\left(y^{2} f\right)^{\prime}=0
$$

so that

$$
f(t)=\frac{c}{\left(\lambda_{1} t^{2}+\lambda_{2} t+\lambda_{3}\right)^{2}}
$$

for some constant numbers $c, \lambda_{1}, \lambda_{2}, \lambda_{3}$.

The relation $f=c y^{-2}$, connecting the potential with symmetries, can be generalized if we consider an arbitrary relation

$$
f=H(y)
$$


for some function $H$. In this case, Lie equation (35) takes the form:

$$
y^{\prime \prime \prime}+2 y^{\prime}\left(y H^{\prime}+2 H\right)=0 \text {. }
$$

In particular, if

$$
f=y+c_{1},
$$

where $c_{1} \in \mathbb{R}$ is a constant number, we obtain the following equation for $f$ :

$$
f^{\prime \prime \prime}+6 f f^{\prime}-2 c_{1} f^{\prime}=0
$$

This equation coincides with the Korteweg-de Vries equation for travelling waves and can be solved explicitly in terms of elliptic functions. Indeed, integrating (42), we obtain

$$
f^{\prime \prime}+3 f^{2}-2 c_{1} f=c_{2},
$$

where $c_{2} \in \mathbb{R}$ is a constant number. Multiplying both sides of (43) by $f^{\prime}$ and integrating, we obtain

$$
\frac{1}{2}\left(f^{\prime}\right)^{2}=c_{1} f^{2}+c_{2} f-f^{3}+c_{3}
$$

$c_{3} \in \mathbb{R}$ being the integrating constant. From (44) it follows that

$$
f(t)=-2 \wp(t)+c_{1} / 3
$$

where $\wp(t)$ is the Weierstrass elliptic function with invariants

$$
g_{2}=c_{2}+\frac{1}{2} c_{1}^{2}, g_{3}=-\frac{1}{2} c_{3}-\frac{1}{18} c_{1} c_{2}-\frac{1}{27} c_{1}^{3}
$$

Thus, Hill equations with potential $f(t)$ of form (45) have shuffling symmetries corresponding to operators of the form:

$$
\Delta=\left(f-c_{1}\right) \partial-\frac{1}{2} f^{\prime}=\left(-2 \wp(t)-\frac{2}{3} c_{1}\right) \partial+\wp^{\prime}(t)
$$

Definition. A potential $f(t)$ is called integrable if equation (35) has at least one nontrivial solution.

Motivations for this definition can be found in chapter 3 .

Consider equation (35) as an equation with respect to $f(t)$. Then we obtain

$$
2\left(y^{2} f\right)^{\prime}+y^{\prime \prime \prime} y=0 .
$$

This implies that all potentials of the form

$$
f(t)=-\frac{1}{2} y(t)^{-2} \int_{t_{0}}^{t} y^{\prime \prime \prime}\left(\tau y(\tau) d \tau, \quad t_{0} \in \mathbb{R},\right.
$$


where $y(t)$ is an arbitrary function, are integrable. For example, putting $y(t)=$ $a \sin (w t)+b \cos (w t)$ in (48), we obtain integrable potentials of the form:

$$
f=\frac{w^{2}}{4}+\frac{c}{(a \sin (w t)+b \cos (w t))^{2}}, \quad w, a, b, c \in \mathbb{R} .
$$

If $y(t)=a e^{\lambda t}+b e^{-\lambda t}$, then

$$
f(t)=-\frac{\lambda^{2}}{4}+\frac{c}{\left(a e^{\lambda t}+b e^{-\lambda t}\right)^{2}}, \quad \lambda, a, b, c \in \mathbb{R} .
$$

In conclusion, let us show a procedure that allows to obtain new integrable potentials from already-known ones. For this purpose, we apply the Lagrange formula to operator (36):

$$
y M(z)+z M(y)=\partial\left(y z^{\prime \prime}-y^{\prime} z^{\prime}+y^{\prime \prime} z+2 f y z\right) .
$$

Putting $z(t)=-y(t)$, for every solution $y(t)$ we obtain

$$
2 y y^{\prime \prime}-\left(y^{\prime}\right)^{2}+2 f y^{2}=c, \quad c \in \mathbb{R} .
$$

This relation can be rewritten as

$$
\left(y^{2}\right)^{\prime \prime}-3\left(y^{\prime}\right)^{2}+2 f y^{2}=c
$$

Therefore, putting

$$
f=\frac{3}{2}\left(\frac{y^{\prime}}{y}\right)^{2}+\frac{1}{2} f_{0}
$$

we obtain the equation for $y(t)$ :

$$
\left(y^{2}\right)^{\prime \prime}+f_{0}(t)\left(y^{2}\right)=0,
$$

This equation can be solved if $f_{0}(t)$ is an integrable potential.

Relations (52) and (53) enable us to construct new integrable potentials starting from already-known ones. The procedure of construction can be described as follows.

1) Let $f_{0}(t)$ be an integrable potential and $z(t)$ a nontrivial solution of the Hill equation

$$
z^{\prime \prime}+f_{0}(t) z=0
$$

2) Then the potential

$$
f(t)=\frac{1}{2} f_{0}(t)+\frac{3}{8}\left(\frac{z^{\prime}}{z}\right)^{2}
$$

is also integrable and the operator

$$
\Delta=\sqrt{z} \partial-\frac{1}{4} \frac{z^{\prime}}{\sqrt{z}} .
$$


determines a shuffling symmetry of the corresponding equation.

If we start with the trivial integrable potential $f_{0}=-v^{2}, v \neq 0$, then after the first iteration we obtain the following integrable potential:

$$
f(t)=-\frac{1}{2} v^{2}+\frac{3}{8} v^{2}\left(\frac{c_{1} e^{v t}-c_{2} e^{-v t}}{c_{1} e^{v t}+c_{2} e^{v t}}\right)^{2}
$$

If $f_{0} \equiv 0$, then we obtain potentials (39).

\section{Remarks.}

1) Note that finding symmetries of Hill equations or, what is the same, finding solutions of equation (35) is equivalent to solving the Hill equations themselves. Thus, we obtain a nontrivial correspondence between differential equations of order 2 and 3 .

2) As a matter of fact, every anti-self-adjoint operator of the form $M=\partial^{3}+$ $a(t) \partial+b(t)$ has form (36). Therefore, the solution space of the corresponding equation $M(h)=0$ is closed with respect to Jacobi bracket (37) and is isomorphic to the Lie algebra $\mathfrak{s l}(2, \mathbb{R})$. A slight improvement of this construction shows that this is also true for every anti-self-adjoint operator of the third order. 


\section{CHAPTER III}

\section{INTEGRALS}

\section{§1. INTEGRALS OF COMPLETELY INTEGRABLE DISTRIBUtions}

1.1 Let $E$ be a completely integrable distribution on a manifold $M$.

\section{Definition.}

1) A function $H \in C^{\infty}(M)$ is called a first integral of the distribution $E$ if $H$ is constant on all integral manifolds of $E$.

2) A function $H \in C^{\infty}\left(J^{k}(\mathbb{R}, M)\right.$ is called a first integral of a system of differential equations $\mathcal{E} \subset J^{k}(\mathbb{R}, M)$ if the restriction of $H$ to the manifold $\mathcal{E}$ is a first integral of the restriction $C(\mathcal{E})$ of the Cartan distribution.

If $E$ is given by differential 1 -forms $w_{1}, \ldots, w_{m}$, i.e. $E=\mathcal{F}\left(w_{1}, \ldots, w_{m}\right)$, then $H \in C^{\infty}(M)$ is a first integral of $E$ if

$$
d H=\lambda_{1} w_{1}+\cdots+\lambda_{m} w_{m}
$$

for some functions $\lambda_{1}, \ldots, \lambda_{m} \in C^{\infty}(M)$. This condition can be rewritten without resorting to functions $\lambda_{1}, \ldots, \lambda_{m}$ :

$$
d H \wedge w_{1} \wedge \cdots \wedge w_{m}=0
$$

Suppose $H_{1}, \ldots, H_{k}$ are first integrals of $E$ and $f\left(x_{1}, \ldots, x_{k}\right)$ is an arbitrary smooth function of $k$ variables. Then the function $H=f\left(H_{1}, \ldots, H_{k}\right)$ is also a first integral of $E$. Therefore, it makes sense to seek only functionally independent first integrals. From (1) it follows that their number does not exceed $m$.

We say that a system of first integrals $H_{1}, \ldots, H_{m}$ of the distribution $E$ is complete if $H_{1}, \ldots, H_{m}$ are functionally independent and $m=\operatorname{codim} E$.

Given a complete system of first integrals, it is possible to find all maximal integral manifolds of $E$ by solving a functional system of the form:

$$
H_{1}=c_{1}, \ldots, H_{m}=c_{m}
$$

where $c_{1}, \ldots, c_{m}$ are constant numbers.

Example. Consider the differential equation $\mathcal{E} \subset J^{1}(\mathbb{R}, \mathbb{R})$ given by

$$
(y+t \cos y) y^{\prime}+\sin y=0
$$

and the function

$$
H\left(t, p_{0}\right)=t \sin p_{0}+\frac{1}{2} p_{0}^{2}
$$


Since the differential 2-form

$$
\begin{aligned}
d H \wedge w_{0}= & \left(\left(p_{0}+t \cos p_{0}\right) d p_{0}+\sin p_{0} d t\right) \wedge\left(d p_{0}-p_{1} d t\right)= \\
& =\left(p_{1}\left(p_{0}+t \cos p_{0}\right)+\sin p_{0}\right) d t \wedge d p_{0}
\end{aligned}
$$

vanishes on $\mathcal{E}$, we see that $H$ is a first integral of $\mathcal{E}$.

1.2. Let $\mathfrak{g}$ be a finite-dimensional Lie algebra of shuffling symmetries of a completely integrable distribution $E$. Suppose that $X_{1}, \ldots, X_{m} \in \operatorname{sym}(E)$ and that the corresponding shuffling symmetries $\widehat{X}_{1}, \ldots, \widehat{X}_{m} \in \operatorname{shuf}(E)$, given by

$$
\widehat{X}_{i}=X_{i} \bmod \operatorname{Char}(E)
$$

form a basis of $\mathfrak{g}$. Furthermore, assume that at every point $a \in M$ the vector fields $X_{1, a}, \ldots, X_{m, a}$ generate the subspace of $\mathrm{T}_{a} M$ complementary to $E(a)$ and that $E=$ $\mathcal{F}\left(w_{1}, \ldots, w_{m}\right)$, where the 1 -forms $w_{1}, \ldots, w_{m}$ are linearly independent at each point of $M$. Then the differential forms $w_{1}, \ldots, w_{m}$ giving the distribution $E$ can be chosen so that

$$
w_{i}\left(X_{j}\right)=w_{i}\left(\widehat{X}_{j}\right)=\delta_{i j}, i, j=1, \ldots, m .
$$

Indeed, let $E=\mathcal{F}\left(\theta_{1}, \ldots, \theta_{m}\right)$ and let the desired forms $w_{1}, \ldots, w_{m}$ have the form

$$
\begin{aligned}
w_{1} & =a_{11} \theta_{1}+\cdots+a_{1 m} \theta_{m} \\
& \vdots \\
w_{m} & =a_{m 1} \theta_{1}+\cdots+a_{m m} \theta_{m}
\end{aligned}
$$

for some nonsingular matrix $A=\left\|a_{i j}\right\|, a_{i j} \in C^{\infty}(M)$. By $S$ denote the matrix

$$
S=\left\|s_{i j}\right\|=\left\|\theta_{i}\left(X_{j}\right)\right\|
$$

The conditions imposed on the vector fields $X_{1}, \ldots, X_{m}$ imply that the matrix $S$ is nonsingular. Further, relations (1) are equivalent to the equality $A \cdot S=I$, so that

$$
A=S^{-1}
$$

and

$$
\left(\begin{array}{c}
w_{1} \\
\vdots \\
w_{m}
\end{array}\right)=S^{-1}\left(\begin{array}{c}
\theta_{1} \\
\vdots \\
\theta_{m}
\end{array}\right) .
$$

1.3. Theorem. Let $\mathfrak{g}$ be a Lie algebra of shuffling symmetries of a completely integrable distribution $E,\left\{\widehat{X}_{1}, \ldots, \widehat{X}_{m}\right\}$ its basis, and $w_{1}, \ldots, w_{m}$ differential 1-forms 
such that $E=\mathcal{F}\left(w_{1}, \ldots, w_{m}\right)$. Suppose that all these objects satisfy conditions of the previous item and that $C_{\alpha, \beta}^{i}, 1 \leqslant i, \alpha, \beta \leqslant m$, are structure constants of $\mathfrak{g}$ :

$$
\left[\widehat{X}_{\alpha}, \widehat{X}_{\beta}\right]=\sum_{i=1}^{m} C_{\alpha, \beta}^{i} \widehat{X}_{i}
$$

Then the forms $w_{1}, \ldots, w_{m}$ satisfy the Maurer-Cartan equations:

$$
d w_{i}+\frac{1}{2} \sum_{\alpha, \beta} C_{\alpha, \beta}^{i} w_{\alpha} \wedge w_{\beta}=0
$$

Proof. By Frobenius' theorem

$$
d w_{i}=\sum_{j=1}^{m} \gamma_{i j} \wedge w_{j}
$$

for some differential 1 -forms $\gamma_{i j}, 1 \leqslant i, j \leqslant m$. Substituting $X_{\alpha}$ in (10), we obtain

$$
\left.X_{\alpha}\right\lrcorner d w_{i}=\sum_{j=1}^{m} \gamma_{i j}\left(X_{\alpha}\right) w_{j}-\gamma_{i \alpha} .
$$

But

$$
\left.L_{X_{\alpha}}\left(d w_{i}\right)=X_{\alpha}\right\lrcorner d w_{i}
$$

Therefore, the differential 1-forms $\left.X_{\alpha}\right\lrcorner d w_{i}$ are linear combinations of the 1-forms $w_{1}, \ldots, w_{m}$, so that

$$
\gamma_{i j}=\sum_{\beta=1}^{m}-A_{\alpha \beta}^{i} w_{\beta}
$$

for some smooth functions $A_{\alpha \beta}^{i}, 1 \leqslant i, \alpha, \beta \leqslant m$. Thus,

$$
d w_{i}=\sum_{\alpha<\beta} A_{\alpha \beta}^{i} w_{\alpha} \wedge w_{\beta} .
$$

This equality implies that

$$
A_{\alpha \beta}^{i}=d w_{i}\left(X_{\alpha}, X_{\beta}\right)
$$

But on other hand,

$$
\begin{gathered}
d w_{i}\left(X_{\alpha}, X_{\beta}\right)=X_{\alpha}\left(w_{i}\left(X_{\beta}\right)\right)-X_{\beta}\left(w_{i}\left(X X_{\alpha}\right)\right)-w_{i}\left(\left[X_{\alpha}, X_{\beta}\right]\right)= \\
=-w_{i}\left(\sum_{j} C_{\alpha \beta}^{j} X_{j}\right)=-C_{\alpha \beta}^{i} .
\end{gathered}
$$

Therefore, $A_{\alpha \beta}^{i}=-C_{\alpha \beta}^{i}$.

1.4. As a corollary of theorem 1.3, we obtain the following result: 
Theorem. Suppose that a distribution $E$ and a Lie algebra $\mathfrak{g}$ of shuffling symmetries of $E$ satisfy conditions of the previous theorem and, in addition, $\mathfrak{g}$ is commutative. Then the differential 1-forms $w_{1}, \ldots, w_{m}$ are closed: $d w_{j}=0$, and therefore first integrals $H_{1}, \ldots, H_{m}$ of $E$ can be found by quadratures:

$$
H_{j}(x)=\int_{a}^{x} w_{j}, \quad j=1, \ldots, m .
$$

Here integration is carried out along arbitrary paths in $M$ connecting a point $x \in M$ with a fixed point $a \in M$.

Remark. In the case of simply connected manifolds $M$ (or manifolds with trivial de Rham cohomology 1-group), first integrals (11) are well-defined. In the general case, formula (11) gives "many-valued" first integrals.

1.5. Let us now revert to the general case considered in 1.3. By $\mathfrak{g}^{(1)}=[\mathfrak{g}, \mathfrak{g}]$ denote the commutator subalgebra of the Lie algebra $\mathfrak{g}$. Suppose $\mathfrak{g}^{(1)} \neq \mathfrak{g}$. Then a basis of $\mathfrak{g}$ can be chosen so that

$$
\widehat{X}_{1}, \ldots, \widehat{X}_{r} \notin \mathfrak{g}^{(1)} \text { and } \widehat{X}_{r+1}, \ldots, \widehat{X}_{m} \in \mathfrak{g}^{(1)}
$$

where $r=\operatorname{codim}_{\mathfrak{g}} \mathfrak{g}^{(1)}$.

Since $\left[\widehat{X}_{i}, \widehat{X}_{j}\right] \in \mathfrak{g}^{(1)}(1 \leqslant i, j \leqslant m)$, we have

$$
C_{i j}^{s}=0, s \leqslant r
$$

Therefore, from the Maurer-Cartan equations it follows that

$$
d w_{j}=0, j \leqslant r
$$

By $H_{1}, \ldots, H_{r}$ denote the first integrals (which may be many-valued) corresponding to the 1 -forms $w_{1}, \ldots, w_{r}$, i.e. $d H_{j}=w_{j}$. Note that these integrals can be found from (11).

Let $c=\left(c_{1}, \ldots, c_{r}\right) \in \mathbb{R}^{r}$ and by $M_{c}$ denote the common level surface of the integrals $H_{1}, \ldots, H_{r}$ :

$$
M_{c}=\left\{x \in M \mid H_{1}(x)=c_{1}, \ldots, H_{r}(x)=c_{r}\right\} .
$$

From linear independence of the 1 -forms $w_{1}, \ldots, w_{r}$ and the implicit -function theorem it follows that $M_{c}$ is a manifold. In addition, since

$$
X_{j}\left(H_{i}\right)=d H_{i}\left(X_{j}\right)=w_{i}\left(X_{j}\right)=0
$$

for $i \leqslant r$ and $j \geqslant r+1$, we see that $M_{c}$ is invariant with respect to the commutator subalgebra. 
So, since $E(a) \subset \mathrm{T}_{a}\left(M_{c}\right)$, we see that the restriction $E_{c}$ of the distribution $E$ to the manifold $M_{c}$ has dimension equal to that of $E$ and can be given by the differential 1forms $w_{r+1}, \ldots, w_{m}$. Moreover, the subalgebra $\mathfrak{g}^{(1)}$ determines an algebra of shuffling symmetries of the distribution $E_{c}$, and the pair $\left(\mathfrak{g}^{(1)}, E_{c}\right)$ satisfies conditions of item 1.2. Therefore, if the subalgebras $\mathfrak{g}^{(2)}=\left[\mathfrak{g}^{(1)}, \mathfrak{g}^{(1)}\right]$ and $\mathfrak{g}^{(1)}$ do not coincide, then the procedure just described can be repeated, and so on. Note that at every new step the codimension of the distribution obtained coincides with the dimension of the corresponding commutator subalgebra.

Define by induction the sequence of derived subalgebras $\mathfrak{g}^{(l)}, l=0,1,2, \ldots$, where

$$
\mathfrak{g}^{(l+1)}=\left[\mathfrak{g}^{(l)}, \mathfrak{g}^{(l)}\right] \text { and } \mathfrak{g}^{(0)}=\mathfrak{g} .
$$

It is clear that $\mathfrak{g}^{(l+1)} \subset \mathfrak{g}^{(l)}, l=0,1,2, \ldots$

Definition. A Lie algebra $\mathfrak{g}$ is called solvable if there exists a natural number $n \geqslant 1$ such that $\mathfrak{g}^{(l)}=0$ for all $l \geqslant n$.

Now suppose that the Lie algebra $\mathfrak{g}$ of shuffling symmetries is solvable. Then the procedure described above enables us to find all first integrals of the distribution $E$ in consecutive order and thereby to find maximal integral manifolds of the distribution. Finally, we obtain the following result:

Theorem. Let $E$ be a completely integrable manifold and $\mathfrak{g} \subset \operatorname{shuf}(E)$ a finitedimensional solvable Lie algebra of shuffling symmetries of $E$. Suppose that $E$ and $\mathfrak{g}$ satisfy the conditions of item 1.2. Then the complete system of first integrals of $E$ can be found with the use of the following two procedures:

a) integration of closed differential 1-forms and

b) solving functional equations (i.e. equations of the form $F(x, y)=0$ ).

\section{§2. LIE-BIANCHI THEOREM}

In this section we are going to apply theorem 1.5 to system of ordinary differential equations.

2.1. First consider systems $\mathcal{E}$ of differential equations of order 1 . Let

$$
\left\{\begin{array}{l}
p_{1}^{1}=F^{1}\left(t, p_{0}^{1}, \ldots, p_{0}^{n}\right) \\
\vdots \\
p_{1}^{n}=F^{n}\left(t, p_{0}^{1}, \ldots, p_{0}^{n}\right)
\end{array}\right.
$$

be a system of this kind and $\mathfrak{g} \subset$ shuf $\mathcal{E}$ a finite-dimensional Lie algebra of shuffling symmetries of system (1). Suppose that the vector fields

$$
\left\{\begin{array}{l}
\widehat{X}_{1}=\varphi_{11} \frac{\partial}{\partial p_{0}^{1}}+\cdots+\varphi_{n 1} \frac{\partial}{\partial p_{0}^{n}} \\
\vdots \\
\widehat{X}_{k}=\varphi_{1 k} \frac{\partial}{\partial p_{0}^{1}}+\cdots+\varphi_{n k} \frac{\partial}{\partial p_{0}^{n}}
\end{array}\right.
$$


form a basis of $\mathfrak{g}$.

In order that $\mathcal{E}$ and $\mathfrak{g}$ satisfy conditions of item 1.2 , it is necessary that $m=n$ and the matrix

$$
S=\left\|\theta_{i}\left(\widehat{X}_{j}\right)\right\|
$$

be non-singular. Here $\theta_{1}, \ldots, \theta_{n}$ are arbitrary 1 -forms that determine the restriction of the Cartan distribution to the system $\mathcal{E}$.

Let $\theta_{1}, \ldots, \theta_{n}$ be the Cartan forms:

$$
\theta_{1}=d p_{0}^{1}-F^{1} d t, \ldots, \theta_{n}=d p_{0}^{n}-F^{n} d t
$$

Then

$$
\theta_{i}\left(\widehat{X}_{j}\right)=\left(d p_{0}^{i}-F^{i} d t\right)\left(\widehat{X}_{j}\right)=\varphi_{i j}
$$

and therefore

$$
S=\left\|S_{i j}\right\|=\left\|\varphi_{i j}\right\|
$$

In this case the forms $\omega_{1}, \ldots, \omega_{n}$ satisfying conditions (3) of item 1.2 can be found from

$$
\left(\begin{array}{c}
\omega_{1} \\
\vdots \\
\omega_{n}
\end{array}\right)=S^{-1}\left(\begin{array}{c}
\theta_{1} \\
\vdots \\
\theta_{n}
\end{array}\right)
$$

Theorem. [Lie, Bianchi]. Suppose that system (1) has a solvable Lie algebra of shuffling symmetries such that its basis has form (2) for $k=n$ and $\operatorname{det}\left\|\varphi_{i j}\right\| \neq 0$. Then the general solution of the system can be found by quadratures.

2.2. Let us describe a procedure of integration of system (1) possessing a solvable Lie algebra $\mathfrak{g}$ of shuffling symmetries.

1) Since $\mathfrak{g}$ is solvable, there exists a sequence of subalgebras

$$
\mathfrak{g}_{0}=\mathfrak{g} \supset \mathfrak{g}_{1} \supset \cdots \supset \mathfrak{g}_{i} \supset \mathfrak{g}_{i+1} \supset \cdots \supset \mathfrak{g}_{n}=\{0\}
$$

such that $\operatorname{codim}_{\mathfrak{g}}\left(\mathfrak{g}_{i}\right)=i, i=0, \ldots, n$, and each subalgebra $\mathfrak{g}_{i+1} \subset \mathfrak{g}_{i}$ is an ideal in $\mathfrak{g}_{i}$. A sequence like this can be constructed as follows.

Let $\mathfrak{g}^{(1)}=[\mathfrak{g}, \mathfrak{g}]$ be the commutator subalgebra. Then every vector subspace $\tilde{\mathfrak{g}}$ of $\mathfrak{g}$ containing $\mathfrak{g}^{(1)}$ is an ideal in $\mathfrak{g}$. Therefore, any subspace of codimension 1 containing $\mathfrak{g}^{(1)}$ can be chosen as $\mathfrak{g}_{1}$. Replacing $\mathfrak{g}$ by $\mathfrak{g}_{1}$, we can similarly construct a Lie algebra $\mathfrak{g}_{2}$, and so on.

2) Let $\left\{\widehat{X}_{1}, \ldots, \widehat{X}_{n}\right\}$ be a basis of $\mathfrak{g}$ such that the vectors $\widehat{X}_{2}, \ldots, \widehat{X}_{n}$ form a basis of the subalgebra $\mathfrak{g}_{1}$, the vectors $\widehat{X}_{3}, \ldots, \widehat{X}_{n}$ form a basis of the subalgebra $\mathfrak{g}_{2}$, 
the vectors $\widehat{X}_{i+1}, \ldots, \widehat{X}_{n}$ form a basis of $\mathfrak{g}_{i}$,

the vector $\widehat{X}_{n}$ forms a basis of $\mathfrak{g}_{n-1}$.

3) Using the Cartan forms $\theta_{1}, \ldots, \theta_{n}$ and relation (4), we find the differential 1forms $\omega_{1}, \ldots, \omega_{n}$ corresponding to the basis $\left\{\widehat{X}_{1}, \ldots, \widehat{X}_{n}\right\}$, i.e. the forms such that

$$
\omega_{i}\left(\widehat{X}_{j}\right)=\delta_{i j}, \quad i, j=1, \ldots, n \text {. }
$$

4) The differential 1 -form $\omega_{1}$ is closed and the function

$$
H_{1}(x)=\int_{a}^{x} \omega_{1}
$$

is a first integral of system (1). The form $\omega_{2}$ is closed on the level surface $M_{c_{1}}$ of $H_{1}$ corresponding to a number $c_{1} \in \mathbb{R}$ and thereby determines the following first integral

$$
H_{2}(\bar{x})=\left.\int_{\bar{a}}^{\bar{x}} \omega_{2}\right|_{M_{c_{1}}}, \quad \bar{a}, \bar{x} \in M_{c_{1}},
$$

where the integration is carried out along a path lying in the manifold $M_{c_{1}}$, and so on.

2.3. Let us give some examples of how to use the procedure.

Example 1. Consider first-order differential equations of the form:

$$
y^{\prime}=F(t, y)
$$

Here $n=1$. Therefore, it is sufficient to require that the equation have a shuffling symmetry of the form:

Then the differential 1-form

$$
\widehat{X}=A\left(t, p_{0}\right) \frac{\partial}{\partial p_{0}}
$$

$$
\omega=\frac{1}{A}\left(d p_{0}-F d t\right)
$$

is closed and the function

$$
H\left(t, p_{0}\right)=\int_{\left(t^{0}, p_{0}^{0}\right)}^{\left(t, p_{0}\right)} \omega
$$

is a first integral of equation (5).

Note that the condition that $\omega$ be closed means that the function $1 / A$ is an integrating factor for the Cartan form

$$
\theta=d p_{0}-F\left(t, p_{0}\right) d t
$$

In the case of homogeneous equations, i.e. equations that have a scaling symmetry, the generating function has the form $\beta p_{0}-\alpha t p_{1}$. Therefore, the integrating factor equals $\left(\beta p_{0}-\alpha t F\right)^{-1}$. 
Example 2. Consider linear systems of differential equations of the form:

$$
\left\{\begin{array}{l}
p_{1}^{1}=F^{11}(t) p_{0}^{1}+\cdots+F^{1 n}(t) p_{0}^{n}+f_{1}(t), \\
\vdots \\
p_{1}^{n}=F^{n 1}(t) p_{0}^{1}+\cdots+F^{n n}(t) p_{0}^{n}+f_{n}(t) .
\end{array}\right.
$$

A vector field

$$
X=\varphi_{1}(t) \frac{\partial}{\partial p_{0}^{1}}+\cdots+\varphi_{n}(t) \frac{\partial}{\partial p_{0}^{n}}
$$

is a shuffling symmetry of system (8) if the vector-valued function

$$
\varphi(t)=\left(\varphi_{1}(t), \ldots, \varphi_{n}(t)\right)
$$

is a solution of the system

$$
\left\{\begin{array}{l}
\dot{\varphi}_{1}(t)=F^{11} \varphi_{1}+\cdots+F^{1 n} \varphi_{n}, \\
\vdots \\
\dot{\varphi}_{n}(t)=F^{n 1} \varphi_{1}+\cdots+F^{n n} \varphi_{n} .
\end{array}\right.
$$

Indeed, in this case system (10) coincides with the Lie equations, which can be easily seen without calculations though: transformations corresponding to field (9) have the form

$$
\varphi:\left(t, p_{0}^{1}, \ldots, p_{0}^{n}\right) \mapsto\left(t, p_{0}^{1}+s \varphi_{1}(t), \ldots, p_{0}^{n}+s \varphi_{n}(t)\right),
$$

and they are symmetries of equations (8) if and only if $\varphi$ is a solution of system (10).

Suppose that vector-valued functions $\varphi^{1}(t), \ldots, \varphi^{n}(t)$ form a fundamental system of solutions of system $(10)$ and $\varphi^{i}(t)=\left(\varphi_{1 i}(t), \ldots, \varphi_{n i}(t)\right)$. Let $M=\left\|\varphi_{i j}(t)\right\|$ be the matrix of this fundamental system. It is obvious that the vector fields $X_{1}, \ldots, X_{n}$ corresponding to the solutions $\varphi^{1}, \ldots, \varphi^{n}$, respectively, commute.

Therefore, the Lie algebra $\mathfrak{g}$ generated by them is commutative and the differential 1-forms $\omega_{1}, \ldots, \omega_{n}$ are closed. The first integrals determined by these forms correspond to solution of system (8) by the method of variation of constants.

2.4. Let us now found out how theorem 1.5 can be modified in the case of ordinary differential equations. We shall start with equations $\mathcal{E}$ of the second order:

$$
p_{2}=F\left(t, p_{0}, p_{1}\right)
$$

In this case the Cartan forms have the form:

$$
\theta_{1}=d p_{0}-p_{1} d t, \quad \theta_{2}=d p_{1}-F d t .
$$

Every shuffling symmetry has the form:

$$
X_{f}=f \frac{\partial}{\partial p_{0}}+\mathcal{D}(f) \frac{\partial}{\partial p_{1}},
$$


where

$$
\mathcal{D}=\frac{\partial}{\partial t}+p_{1} \frac{\partial}{\partial p_{0}}+F \frac{\partial}{\partial p_{1}}
$$

and $f=f\left(t, p_{0}, p_{1}\right)$ is an arbitrary function. Then

$$
\theta_{1}\left(X_{f}\right)=f, \quad \theta_{2}\left(X_{f}\right)=\mathcal{D}(f)
$$

Let $\mathfrak{g} \subset \operatorname{shuf}(\mathcal{E})$ be a two-dimensional Lie algebra of shuffling symmetries of $\mathcal{E}$ and $\left\{X_{f_{1}}, X_{f_{2}}\right\}$ its basis. In order to apply theorem 1.5, it is necessary that the following matrix be non-singular:

$$
S=\left[\begin{array}{cc}
f_{1} & f_{2} \\
\mathcal{D}\left(f_{1}\right) & \mathcal{D}\left(f_{2}\right)
\end{array}\right] .
$$

(This matrix is called the generalized Wronski matrix.) If $\operatorname{det} S \neq 0$, then the forms $\omega_{1}$ and $\omega_{2}$ can be found from

$$
\left(\begin{array}{l}
\omega_{1} \\
\omega_{2}
\end{array}\right)=\frac{1}{\operatorname{det} S}\left[\begin{array}{cc}
\mathcal{D}\left(f_{2}\right) & -f_{2} \\
-\mathcal{D}\left(f_{1}\right) & f_{1}
\end{array}\right]\left(\begin{array}{l}
\theta_{1} \\
\theta_{2}
\end{array}\right)
$$

so that

$$
\begin{aligned}
& \omega_{1}=\frac{\mathcal{D}\left(f_{2}\right) \theta_{1}-f_{2} \theta_{2}}{\mathcal{D}\left(f_{2}\right) f_{1}-f_{2} \mathcal{D}\left(f_{1}\right)}, \\
& \omega_{2}=\frac{-\mathcal{D}\left(f_{1}\right) \theta_{1}+f_{1} \theta_{2}}{\mathcal{D}\left(f_{2}\right) f_{1}-f_{2} \mathcal{D}\left(f_{1}\right)},
\end{aligned}
$$

or

$$
\begin{aligned}
& \omega_{1}=\frac{\mathcal{D}\left(f_{2}\right)}{d} d p_{0}-\frac{f_{2}}{d} d p_{1}+\frac{F f_{2}-p_{1} \mathcal{D}\left(f_{2}\right)}{d} d t \\
& \omega_{2}=-\frac{\mathcal{D}\left(f_{1}\right)}{d} d p_{0}+\frac{f_{1}}{d} d p_{1}+\frac{-F f_{1}-p_{1} \mathcal{D}\left(f_{1}\right)}{d} d t,
\end{aligned}
$$

where $d=\operatorname{det} S=f_{1} \mathcal{D}\left(f_{2}\right)-f_{2} \mathcal{D}\left(f_{1}\right)$.

There are two types of 2-dimensional Lie algebras:

a) commutative Lie algebras: $\left[X_{f_{1}}, X_{f_{2}}\right]=0$, and

b) solvable but not commutative ones and it can be assumed that

$$
\left[X_{f_{1}}, X_{f_{2}}\right]=X_{f_{2}} \text {. }
$$

Therefore, in the case of second-order differential equations theorem 1.5 takes the following form:

Theorem. [Lie, Bianchi]. Suppose that second-order equation (11) has a twodimensional solvable Lie algebra $\mathfrak{g}$ of shuffling symmetries such that Wronski matrix (15) is non-singular. Let $\left\{X_{f_{1}}, X_{f_{2}}\right\}$ be a basis of $\mathfrak{g}$ such that one of conditions (18) is satisfied. Then 
a) if $\mathfrak{g}$ is commutative, then the differential 1-forms $\omega_{1}$ and $\omega_{2}$ (see (17)) are closed and give two independent first integrals:

$$
H_{1}(x)=\int \omega_{1}, \quad H_{2}(x)=\int \omega_{2}
$$

b) if $\mathfrak{g}$ is solvable but not commutative, then the differential 1-form $\omega_{1}$ is closed and gives the first integral

$$
H_{1}(x)=\int \omega_{1}
$$

the 1-form $\omega_{2}$ is closed on a level surface of $H_{1}$ and gives an independent first integral of equation (11).

2.5. Example 1. Consider linear differential equations of the second order:

$$
p_{2}=A(t) p_{1}+B(t) p_{0}+C(t) .
$$

Then, as in example 2.3.(2), a function $f=f(t)$ is a generating function of a symmetry if and only if $f$ satisfies the homogeneous differential equation

$$
f^{\prime \prime}=A(t) f^{\prime}+B(t) f .
$$

Let $\left\{f_{1}, f_{2}\right\}$ be a fundamental system of solutions of equation (20). It is obvious that the vector fields $X_{f_{1}}$ and $X_{f_{2}}$ commute and therefore form a two-dimensional commutative Lie algebra of symmetries of (19). Hence, the differential 1-forms

$$
\begin{aligned}
& \omega_{1}=\frac{f_{2}^{\prime}}{d} d p_{0}-\frac{f_{2}}{d} d p_{1}+\frac{\left(A-f_{2}^{\prime}\right) p_{1}-B p_{0} f_{2}+C f_{2}}{d} d t \\
& \omega_{2}=-\frac{f_{1}^{\prime}}{d} d p_{0}+\frac{f_{1}}{d} d p_{1}+\frac{\left(f_{1}^{\prime}-A\right) p_{1}-B p_{0} f_{1}-C f_{1}}{d} d t,
\end{aligned}
$$

where

$$
d=\left|\begin{array}{ll}
f_{1} & f_{2} \\
f_{1}^{\prime} & f_{2}^{\prime}
\end{array}\right|
$$

is the Wronski determinant, are closed. Thus, the complete system of solutions of a non-homogeneous equation is determined by a fundamental system of solutions of the corresponding homogeneous equation. This well-known fact can also be proved by the method of variation of constants.

2.6. Example 2. Consider the Hill equation:

$$
y^{\prime \prime}-F(t) y=0 \text {. }
$$

This is a homogeneous linear equation. Therefore, it always has the scaling symmetry $\left(t, p_{0}\right) \mapsto\left(t, \lambda p_{0}\right)$. The generating function of the corresponding infinitesimal scaling symmetry has the form:

$$
f_{1}=p_{0}
$$


Assume that equation (22) has a linear symmetry with a generating function of the form:

$$
f_{2}=A(t) p_{1}+B(t) p_{0}
$$

Then

$$
\left[f_{1}, f_{2}\right]=0
$$

and the Lie algebra $\mathfrak{g}$ generated by the vector fields $X_{f_{1}}$ and $X_{f_{2}}$ is commutative. Hence, the differential 1-forms

$$
\begin{gathered}
\omega_{1}=\frac{\left(A^{\prime}+B\right) p_{1}+\left(B^{\prime}+F A\right) p_{0}}{d} d p_{0}-\frac{A p_{1}+B p_{0}}{d} d p_{1}+ \\
+\frac{F B p_{0}^{2}-B^{\prime} p_{1} p_{0}-\left(A^{\prime}+B\right) p_{1}^{2}}{d} d t, \\
\omega_{2}=-\frac{p_{1}}{d} d p_{0}+\frac{p_{0}}{d} d p_{1}+\frac{-B p_{0}^{2}-A p_{1} p_{0}+p_{1}^{2}}{d} d t,
\end{gathered}
$$

where $d=\left(B^{\prime}+F A\right) p_{0}^{2}+A^{\prime} p_{0} p_{1}-A p_{1}^{2}$ is the Wronski determinant corresponding to the given symmetries, are closed and therefore give a complete system of first integrals for equation (22).

Using the results of item 5.9, chapter 2 , we obtain the following result:

Theorem. 1) The Hill equation is integrable by quadratures for the following potentials:

(i) $\quad F(t)=\frac{c}{\left(a_{1} t^{2}+a_{2} t+a_{3}\right)^{2}}, \quad a_{1}, a_{2}, a_{3}, c \in \mathbb{R} ;$

(ii) $F(t)=-2 \wp(t)+\frac{c}{3}, \quad c \in \mathbb{R}$;

(iii) $F(t)=\frac{\omega^{2}}{4}+\frac{c}{(a \sin (\omega t)+b \cos (\omega t))^{2}}, \quad F(t)=-\frac{\lambda^{2}}{4}+\frac{c}{\left(a \mathrm{e}^{\lambda t}+b \mathrm{e}^{-\lambda t}\right)^{2}}$, $a, b, c, \lambda, \omega \in \mathbb{R}$

(iv) $F(t)=\frac{1}{2} v^{2}+\frac{3}{8} v^{2}\left(\frac{c_{1} \mathrm{e}^{v t}-c_{2} \mathrm{e}^{-v t}}{c_{1} \mathrm{e}^{v t}+c_{2} \mathrm{e}^{-v t}}\right)^{2}, \quad c_{1}, c_{2}, v \in \mathbb{R}$.

The following functions can be chosen as a function $f_{2}$ in these cases:

(i) $f_{2}=\left(a_{1} t^{2}+a_{2} t+a_{3}\right) p_{1}-\left(a_{1} t+\frac{a_{2}}{2}\right) p_{0} ;$

(ii) $f_{2}=\left(-2 \wp(t)-\frac{2}{3} c\right) p_{1}+\wp^{\prime} p_{0}$;

(iii) $f_{2}=\left(a \sin (\omega t)+b \cos (\omega t) p_{1}-\frac{\omega}{2}(a \cos (\omega t)-b \sin (\omega t)) p_{0}\right.$

and

$$
f_{2}=\left(a \mathrm{e}^{\lambda t}+b \mathrm{e}^{-\lambda t}\right) p_{1}-\frac{\lambda}{2}\left(a \mathrm{e}^{\lambda t}-b \mathrm{e}^{-\lambda t}\right) p_{0}
$$




$$
\text { (iv) } f_{2}=p_{1} \sqrt{c_{1} \mathrm{e}^{v t}+c_{2} \mathrm{e}^{-v t}}-\frac{v\left(c_{1} \mathrm{e}^{v t}-c_{2} \mathrm{e}^{-v t}\right)}{4 \sqrt{c_{1} \mathrm{e}^{v t}+c_{2} \mathrm{e}^{-v t}}} p_{0}
$$

2) If a potential $F(t)$ is integrable by means of symmetries (24), then the potential

$$
F_{H}(t)=\frac{1}{2} F(t)+\frac{3}{8}\left(\frac{z^{\prime}}{z}\right)^{2}
$$

where $z(t)$ is a nontrivial solution of the Hill equation with potential $F(t)$, is also integrable and the following function can be chosen for $f_{2}$ :

$$
f_{2}=\sqrt{z} p_{1}-\frac{1}{4} \frac{z^{\prime}}{\sqrt{z}} p_{0}
$$

2.7. Example 3. Consider second-order equations of the form:

$$
y^{\prime \prime}=y^{\prime}+\varphi(y)
$$

where functions $\varphi(y)$ are those listed in item 4.5, chapter 2. Shuffling symmetries with generating functions $f_{1}$ and $f_{2}$ described in that theorem form solvable Lie algebras:

$$
\begin{aligned}
& {\left[f_{1}, f_{2}\right]=k f_{2} \text { in case }(i) \text { and }} \\
& {\left[f_{1}, f_{2}\right]=-f_{2} \text { in case }(i i) .}
\end{aligned}
$$

Choosing a new basis:

$$
\begin{aligned}
& \bar{f}_{1}=\frac{1}{k} f_{1}, \bar{f}_{2}=f_{2} \text { in case }(i) ; \\
& \bar{f}_{1}=-f_{1}, \bar{f}_{2}=f_{2} \text { in case }(i i),
\end{aligned}
$$

we arrive at the situation described in theorem 2.4.

Thus, the differential 1-forms

(i)

$$
\begin{aligned}
\omega_{1}^{(i)}=\frac{k f_{2}+\left(\varphi+\frac{1-k}{2} p_{1}\right) \mathrm{e}^{k t}}{d^{(i)}} d p_{0}-\frac{f_{2}}{d^{(i)}} d p_{1}+ & \\
& +\frac{\left(\varphi-(k-1) p_{1}\right) f_{2}-p_{1}\left(\varphi+\frac{1-k}{2} p_{1}\right) \mathrm{e}^{k t}}{d^{(i)}} d t
\end{aligned}
$$

(ii)

$$
\omega_{1}^{(i i)}=\frac{-f_{2}+\left(\varphi+p_{1}\right) \mathrm{e}^{-t}}{d^{(i i)}} d p_{0}-\frac{f_{2}}{d^{(i i)}} d p_{1}+\frac{-\varphi f_{2}-p_{1}\left(\varphi+p_{1}\right) \mathrm{e}^{-t}}{d^{(i i)}} d t
$$

are closed in cases $(i)$ and $(i i)$, respectively, and give the first integrals $H_{1}^{(i)}$ and $H_{1}^{(i i)}$ of equation (25). The differential 1-forms

(i)

$$
\omega_{2}^{(i)}=-\frac{\left(p_{1}+\varphi\right)}{d^{(i)}} d p_{0}+\frac{p_{1}}{d^{(i)}} d p_{1}
$$


(ii)

$$
\omega_{2}^{(i i)}=-\frac{\left(p_{1}+\varphi\right)}{d^{(i i)}} d p_{0}+\frac{p_{1}}{d^{(i i)}} d p_{1}
$$

are closed on level surfaces of the first integrals $H_{1}^{(i)}$ and $H_{1}^{(i i)}$ respectively. Here $d^{(i)}$ and $d^{(i i)}$ are the Wronski determinants corresponding to cases $(i)$ and (ii) respectively.

2.8. For $k$-th order ordinary differential equations

$$
y^{(k)}=F\left(t, y, y^{\prime}, \ldots, y^{(k-1)}\right)
$$

Lie-Bianchi theorem takes the following form:

Theorem. Suppose that differential equation (26) has a $k$-dimensional solvable Lie algebra $\mathfrak{g}$ of shuffling symmetries and let $\left\{\varphi_{1}, \ldots, \varphi_{k}\right\}$ be a basis of $\mathfrak{g}$. Now suppose that the determinant of the corresponding Wronski matrix

$$
W=\operatorname{det}\left|\begin{array}{ccc}
\varphi_{1} & \ldots & \varphi_{k} \\
\mathcal{D} \varphi_{1} & \ldots & \mathcal{D} \varphi_{k} \\
\vdots & & \\
\mathcal{D}^{k-1} \varphi_{1} & \ldots & \mathcal{D}^{k-1} v_{k}
\end{array}\right|
$$

does not vanish. Then equation (26) has $k$ independent first integrals that can be found by means of the method described in 2.2 .

Proof. It is sufficient to note that if

$$
\theta_{0}=d p_{0}-p_{1} d t, \ldots, \theta_{k-2}=d p_{k-2}-p_{k-1} d t, \theta_{k-1}=d p_{k-1}-F d t
$$

then

$$
\theta_{i}\left(X_{\varphi}\right)=\mathcal{D}^{i} \varphi
$$

where

$$
\mathcal{D}=\frac{\partial}{\partial t}+p_{1} \frac{\partial}{\partial p_{0}}+\cdots+p_{k-1} \frac{\partial}{\partial p_{k-2}}+F \frac{\partial}{\partial p_{k-1}}
$$

is the operator of total differentiation. Therefore, the matrix $S$ involved in 2.1 coincides with Wronski matrix (27).

2.9. Remark. Usually-suggested methods of solving differential equations with the use of symmetries consist in lowering of the order of a differential equation to be integrated and assume that first integrals are already known.

More exactly, let $X$ be a point symmetry, i.e. a vector field on $\mathbb{R} \times M$. Suppose that there exist local coordinates such that

$$
X=\frac{\partial}{\partial p_{0}^{1}}
$$


Then equations, say, first-order equations possessing this symmetry can be written as

$$
\left\{\begin{array}{l}
F_{1}\left(t, p_{0}^{2}, \ldots, p_{0}^{n}, p_{1}^{1}, \ldots, p_{n}^{1}\right)=0 \\
F_{i}\left(t, p_{0}^{2}, \ldots, p_{0}^{n}, p_{1}^{2}, \ldots, p_{n}^{2}\right)=0, \quad i=2, \ldots, n .
\end{array}\right.
$$

Therefore, it is sufficient to integrate the last $n-1$ equations with respect to $p_{0}^{2}, \ldots, p_{n}^{2}$ and then turn back to the first equation and find $y^{1}$.

Similarly, for a single ordinary differential equation possessing a contact symmetry $X_{f}$, there exists a canonical coordinate system such that $f=1$ and

$$
X_{f}=\frac{\partial}{\partial p_{0}}
$$

Therefore, a $k$-th order differential equation possessing this symmetry can be written as

$$
F\left(t, p_{1}, \ldots, p_{k}\right)=0 \text {. }
$$

The last equation can be reduced to a $(k-1)$-order differential equation.

\section{§3. Integrals of Distributions (The General CASE)}

In this section we are going to generalize results of $\S 1$ to the case of arbitrary transitive actions of Lie algebras of shuffling symmetries.

3.1. Let $E=\mathcal{F}\left(\omega_{1}, \ldots, \omega_{m}\right)$ be a completely integrable distribution on a manifold $M$ and $\mathfrak{g} \subset \operatorname{shuf} E$ a Lie algebra of shuffling symmetries, $\operatorname{dim} \mathfrak{g}=k$. Let $X \in \mathfrak{g}$ be a shuffling symmetry. The value $X_{a}$ of $X$ at a point $a \in M$ is the element of the factor space $\mathrm{T}_{a} M / E(a)$ determined by the value of any representative of $X$ in the Lie algebra $\operatorname{sym}(E)$ of infinitesimal symmetries.

Definition. We say that the Lie algebra $\mathfrak{g}$ acts transitively on the set of maximal integral manifolds of the distribution $E$ if for any basis $\left\{X_{1}, \ldots, X_{k}\right\}$ of $\mathfrak{g}$ the values $X_{1, a}, \ldots, X_{k, a}$ of the vector fields $X_{1}, \ldots, X_{k}$ at each point $a \in M$ generate the factor space $\mathrm{T}_{a} M / E(a)$.

Note that if $\mathfrak{g}$ acts transitively, then $\operatorname{dim} \mathfrak{g} \geqslant \operatorname{codim} E$.

At each point $a \in M$, by $\mathfrak{g}_{a}$ denote the set

$$
\mathfrak{g}_{a}=\left\{X \in \mathfrak{g} \mid X_{a}=0\right\}
$$

Thus, an infinitesimal symmetry $\bar{X}$ generates a shuffling symmetry lying in $\mathfrak{g}_{a}$ if and only if $\bar{X}_{a} \in E(a)$. This observation shows that the following statement is true:

3.2. Proposition. $\mathfrak{g}_{a}$ is a subalgebra of $\mathfrak{g}$.

Proof. Let $X, Y \in \mathfrak{g}_{a}$ and let $\bar{X}, \bar{Y} \in \operatorname{sym}(E)$ be representatives of $X$ and $Y$ respectively. 
We have

$$
d \omega_{j}=\sum_{i} \gamma_{j i} \wedge \omega_{i}
$$

for some 1 -forms $\gamma_{j i}$. Then since

$$
\omega_{j}([\bar{X}, \bar{Y}])=-d \omega_{j}(\bar{X}, \bar{Y})+\bar{X}\left(\omega_{j}(\bar{Y})\right)+\bar{Y}\left(\omega_{j}(\bar{X})\right),
$$

we obtain

$$
\omega_{j}\left([\bar{X}, \bar{Y}]_{a}\right)=0
$$

if $\omega_{1}\left(\bar{X}_{a}\right)=\cdots=\omega_{m}\left(\bar{X}_{a}\right)=\omega_{1}\left(\bar{Y}_{a}\right)=\ldots \omega_{m}\left(\bar{Y}_{a}\right)=0$. Therefore,

$$
[\bar{X}, \bar{Y}]_{a} \in E(a)
$$

so that $[\bar{X}, \bar{Y}] \in \mathfrak{g}_{a}$.

The subalgebra $\mathfrak{g}_{a}$ is called the stability subalgebra of the point $a$.

3.3. Suppose that the action of $\mathfrak{g}$ is transitive. Then at each point $a \in M$ the following conditions hold:

$$
\begin{gathered}
\mathfrak{g} / \mathfrak{g}_{a}=\mathrm{T}_{a} M / E(a), \\
\left(\mathrm{T}_{a} M / E(a)\right)^{*}=\operatorname{Ann} E(a), \\
\left(\mathfrak{g} / \mathfrak{g}_{a}\right)^{*}=\operatorname{Ann} \mathfrak{g}_{a} .
\end{gathered}
$$

Hence,

$$
\operatorname{Ann} E(a)=\operatorname{Ann} \mathfrak{g}_{a} \subset \mathfrak{g}^{*} .
$$

Denote by $V$ an arbitrary subspace of $\mathfrak{g}$ transversal to $\mathfrak{g}_{a}$, so that

$$
\mathfrak{g}=\mathfrak{g}_{a} \oplus V
$$

Let $\left\{X_{1}, \ldots, X_{m}\right\}$ be a basis of $V$. Then for every point $b \in M$ lying in some neighborhood $\mathcal{O}$ of the point $a$, the values $X_{1, b}, \ldots, X_{m, b}$ generate the factor space $\mathrm{T}_{b} M / E(b)$. As in 1.2, let us choose the differential 1-forms $\omega_{1}, \ldots, \omega_{m}$ determining the distribution $E$ so that

$$
\omega_{i}\left(X_{j}\right)=\delta_{i j}, \quad i, j=1, \ldots, m
$$

in the neighborhood $\mathcal{O}$. Then

$$
\left\{\begin{array}{l}
d \omega_{i}\left(X_{l}, X_{r}\right)=\omega_{i}\left(\left[X_{r}, X_{l}\right]\right) \\
d \omega\left(Z_{1}, Z_{2}\right)=0 \\
d \omega_{i}\left(X_{l}, Z_{1}\right)=0
\end{array}\right.
$$

for all $i, l, r=1, \ldots, m$ and all vector fields $Z_{1}, Z_{2} \in \mathcal{D}(E)$.

Indeed, in order to prove these relations, it is sufficient to make use of (1). 
Further, relation (2) implies that the differential forms $\omega_{1}, \ldots, \omega_{m}$ can be considered as elements $\hat{\omega}_{1}, \ldots, \hat{\omega}_{m}$ of the space $\mathfrak{g}^{*}$ if we make use of $(4)$ and assume that $\hat{\omega}_{i}(X)=0$ for all $X \in \mathfrak{g}_{a}$. From (6) it follows that if $\hat{\omega}_{i} \in \operatorname{Ann}[\mathfrak{g}, \mathfrak{g}] \subset \mathfrak{g}^{*}$, then the corresponding differential form $\omega_{i}$ is closed and therefore determines a first integral of the distribution.

Thus we obtain $m_{1}$ independent first integrals of $E$, where

$$
m_{1}=\operatorname{codim}_{\mathfrak{g}}\left(\mathfrak{g}_{a}+[\mathfrak{g}, \mathfrak{g}]\right)
$$

In order to explicitly describe these integrals, let us choose subspaces $V_{1}, V_{2} \subset \mathfrak{g}$ so that

$$
V=V_{1} \oplus V_{2}, \quad V_{2} \oplus\left(\mathfrak{g}_{a}+[\mathfrak{g}, \mathfrak{g}]\right)=\mathfrak{g}
$$

and

$$
V_{1}+\left(\mathfrak{g}_{a} \cap[\mathfrak{g}, \mathfrak{g}]\right)=[\mathfrak{g}, \mathfrak{g}] .
$$

Let $\left\{X_{1}, \ldots, X_{k}\right\}$ be a basis of $\mathfrak{g}$ such that the vectors $X_{1}, \ldots, X_{m-m_{1}-1}$ form a basis of $V_{1}$ and the vectors $X_{m-m_{1}}, \ldots, X_{m}$ form a basis of $V_{2}$. Then

$$
d \omega_{j}=0
$$

for $j=m-m_{1}, \ldots, m$.

Put

$$
\mathfrak{g}^{(1)}=\mathfrak{g}_{a}+[\mathfrak{g}, \mathfrak{g}]
$$

Note that the algebra $\mathfrak{g}^{(1)}$ does not depend on a choice of the point $a \in M$, since

$$
\operatorname{Ad}_{h}\left(\mathfrak{g}_{a}\right) \subset \mathfrak{g}_{a}+[\mathfrak{g}, \mathfrak{g}]
$$

As before, consider common level surfaces of the first integrals

$$
H_{j}(x)=\int_{a}^{x} \omega_{j}, \quad j=m-m_{1}, \ldots, m
$$

and the new pair $\left(\mathfrak{g}^{(1)}, \mathfrak{g}_{a}\right)$.

Let us generalize the definition of a solvable Lie algebra given in $\S$ :

Definition. Let $\mathfrak{g}$ be a Lie algebra and $\mathfrak{g}_{a}$ its subalgebra. The pair $\left(\mathfrak{g}, \mathfrak{g}_{a}\right)$ is called solvable if the sequence of subalgebras

$$
\mathfrak{g}^{(0)}=\mathfrak{g}, \mathfrak{g}^{(1)}=\mathfrak{g}_{0}+[\mathfrak{g}, \mathfrak{g}], \ldots, \mathfrak{g}^{(l+1)}=g_{0}+\left[\mathfrak{g}_{0}^{(l)}, g_{0}^{(l)}\right], \ldots
$$

converges to $\mathfrak{g}_{0}$, i.e. $\mathfrak{g}^{(i)}=\mathfrak{g}_{0}$ starting from some number $i$.

Summarizing all that has been said in this item, we obtain the following result: 
Theorem. Suppose that a completely integrable distribution $E$ has a transitive Lie algebra $\mathfrak{g} \subset$ shuf $E$ of shuffling symmetries and $\mathfrak{g}_{0} \subset \mathfrak{g}$ is a stability subalgebra of some point. If the pair $\left(\mathfrak{g}, \mathfrak{g}_{0}\right)$ is solvable, then first integrals of the distribution can be found by consecutive quadratures.

3.4. Let us apply the result just obtained to integration of actions of Lie algebras on smooth manifolds.

Let $\mathfrak{g}$ be a transitive Lie algebra of vector fields on a contact manifold $M$ and $\mathfrak{g}_{a} \subset \mathfrak{g}$ the stability subalgebra of some fixed point $a \in M$.

Every subalgebra $L \subset \mathfrak{g}$ such that $L \supset \mathfrak{g}_{a}$ determines a distribution (which will also be denoted by $L$ ).

Indeed, denote by $G$ the connected Lie group corresponding to the Lie algebra $\mathfrak{g}$. Since elements of $\mathfrak{g}$ are vector fields on $M$, we see that elements of $G$ are diffeomorphisms of $M$. The group $G$ acts transitively on $M$. Define the distribution $L$ as follows: for $b \in M, L(b)$ is the image $g_{*}(L) \subset \mathrm{T}_{b} M$ of the subalgebra $L$ by the differential $g_{*}$ of any transformation $g \in G$ such that $g(a)=b$. Since $L$ is closed with respect to commutation with elements of $\mathfrak{g}_{a}$, we see that the space $L(b)$ does not depend on a choice of $g \in G$.

The corresponding Lie algebra of shuffling symmetries of the distribution $L$ has the form:

$$
\operatorname{shuf}_{\mathfrak{g}} L=N(L) / L
$$

where $N(L)$ is the normalizer of $L$ in $\mathfrak{g}$ :

$$
N(L)=\{X \in \mathfrak{g} \mid[X, L] \subset L\}
$$

Conditions for this algebra to act transitively on maximal integral manifolds of the distribution $L$ have the form:

$$
\operatorname{dim} N(L) / L \geqslant \operatorname{dim} \mathfrak{g} / \mathfrak{g}_{0}-\operatorname{dim} L / \mathfrak{g}_{0}=\operatorname{dim} \mathfrak{g} / L .
$$

Thus, the Lie algebra $\operatorname{shuf}_{\mathfrak{g}} L$ is transitive if and only if $N(L)=\mathfrak{g}$, i.e. if $L$ is an ideal in $\mathfrak{g}$.

In order to apply theorem 3.3 in this case, we must require that the factor algebra $\mathfrak{g} / L$ be solvable. Finally, we obtain the following result:

Theorem. Let $\mathfrak{g}$ be a transitive Lie algebra of vector fields on a manifold $M, \mathfrak{g}_{0} \subset \mathfrak{g}$ the stability subalgebra of some fixed point of $M$, and $L$ an ideal in $\mathfrak{g}$ such that $\mathfrak{g}_{0} \subset L$. Suppose that the factor algebra $\mathfrak{g} / L$ is solvable. Then the complete system of first integrals of the completely integrable distribution generated by $L$ can be found by consecutive quadratures.

3.5. Let us apply the last theorem to a distribution defined by a vector field $X$ on a Lie group $M=G$. Let $\mathfrak{g}$ be the Lie algebra of $G$. The subalgebra $L=\mathbb{R} X \subset \mathfrak{g}$ is an ideal in $\mathfrak{g}$ if and only if $X$ is an eigenvector for all operators ad $Y, Y \in \mathfrak{g}$.

In particular, this observation implies that every effective transitive action of a solvable Lie algebra is integrable by means of quadratures. 


\section{$\S 4$. DifFERENTIAL INVARIANTS}

In order to integrate equations by means of symmetries, it is necessary to know a sufficiently large algebra of shuffling symmetries. The problem of finding this algebra, i.e. of solving a system of Lie equations, is also not a simple problem. However, this problem can be approached from the opposite side: given a Lie algebra $\mathfrak{g}$, to describe all differential equations such that $\mathfrak{g}$ can be considered as their algebra of shuffling symmetries. The present section is devoted to this approach.

4.1. Suppose that a Lie algebra $\mathfrak{g}$ is embedded as a subalgebra into the Lie algebra $\mathcal{D}(M \times \mathbb{R})$ of vector fields on $M \times \mathbb{R}$. As in chapter 2 , by $X^{(k)}$ we denote the $k$-th prolongation of a vector field $X$ to the space $J^{k}(\mathbb{R}, M)$ of $k$-jets of parametrized curves.

The correspondence $X \mapsto X^{(k)}$ gives an embedding of $\mathfrak{g}$ into $\mathcal{D}\left(J^{k}(\mathbb{R}, M)\right)$. By $\mathfrak{g}^{k}$ denote the image of the embedding.

Definition. A function $f \in C^{\infty}\left(J^{k}(\mathbb{R}, M)\right)$ is called a $k$-th order differential invariant of the Lie algebra $\mathfrak{g}$ if

$$
X^{(k)}(f)=0
$$

for all prolongations $X^{(k)} \in \mathfrak{g}^{k}$.

This definition can be formulated in a different way. Let $G$ be a connected Lie group of transformations of $\mathbb{R} \times M$ such that $\mathfrak{g}$ is its Lie algebra. For a $g \in G$, denote by $\mathfrak{g}^{(k)}$ the prolongation of the transformation $g: \mathbb{R} \times M \rightarrow \mathbb{R} \times M$ to transformations of the space $J^{k}(\mathbb{R}, M)$. Actually, the transformation $\mathfrak{g}^{(k)}$ is defined on an open and everywhere dense part of the manifold $J^{k}(\mathbb{R}, M)$ However, with the motivation of the definition in mind, for simplicity sake we shall assume that $\mathfrak{g}^{(k)}$ is completely defined. Then for each point $a \in J^{k}(\mathbb{R}, M)$ consider its orbit with respect to the group $G$ :

$$
G^{(k)}(a)=\left\{g^{(k)}(a) \mid \forall g \in G\right\}
$$

Thus, we obtain the division of the manifold $J^{k}(\mathbb{R}, M)$ into orbits of the group $G$.

Differential invariants are functions on $J^{k}(\mathbb{R}, M)$ constant on each orbit.

4.2. Denote by $I_{k}(\mathfrak{g}) \subset C^{\infty}\left(J^{k}(\mathbb{R}, M)\right)$ the set of all differential invariants of order $k$. The following result is immediate from the definition:

Proposition. The set $I_{k}(\mathfrak{g})$ is a subalgebra of the algebra of smooth functions on $J^{k}(\mathbb{R}, M)$. In addition, if $f_{1}, \ldots, f_{r} \in I_{k}(\mathfrak{g})$ and $F\left(x_{1}, \ldots, x_{r}\right)$ is an arbitrary smooth function of $r$ variables, then $F\left(f_{1}, \ldots, f_{r}\right) \in I_{k}(\mathfrak{g})$.

Note that if a system $\mathcal{E} \subset J^{k}(\mathbb{R}, M)$ of differential equations is given by invariants $f_{1}, \ldots, f_{N} \in I_{k}$, i.e.

$$
\mathcal{E}=\left\{f_{1}=0\right\} \cap \cdots \cap\left\{f_{N}=0\right\}
$$

then $\mathfrak{g} \subset \operatorname{sym} \mathcal{E}$.

Thus, the definition of differential invariants allows to construct a number of classes of differential equations with a given symmetry algebra.

4.3 Let us give some examples of direct calculating algebras of invariants. 
Example 1. Let $M=\mathbb{R}$ and let $\mathfrak{g}=\mathfrak{s l}(2, \mathbb{R})$ be the Lie algebra of vector fields on $J^{0}(\mathbb{R}, \mathbb{R})=\mathbb{R}^{2}$ realized by the fields

$$
X_{1}=\frac{\partial}{\partial p_{0}}, X_{2}=p_{0} \frac{\partial}{\partial p_{0}}, X_{3}=p_{0}^{2} \frac{\partial}{\partial p_{0}}
$$

The corresponding Lie group $\operatorname{SL}(2, \mathbb{R})$ is the group of linear fractional transformations

$$
g\left(t, p_{0}\right)=\left(t, \frac{a_{11} p_{0}+a_{12}}{a_{21} p_{0}+a_{22}}\right),
$$

where $\left[\begin{array}{ll}a_{11} & a_{12} \\ a_{21} & a_{22}\end{array}\right] \in \mathrm{SL}(2, \mathbb{R})$.

Differential invariants of order 0 are functions $f\left(t, p_{0}\right)$ such that $f\left(g\left(t, p_{0}\right)\right)=$ $f\left(t, p_{0}\right)$ for all transformations $g$ of form $(2)$. It follows that $I_{0}(\mathfrak{s l}(2, \mathbb{R}))$ is the set of functions independent of $p_{0}$.

Definition. We say that $k$-th order differential invariants $f_{1}, \ldots, f_{r}$ form a (local) basis of $I_{k}(\mathfrak{g})$ if every differential invariant $f \in I_{k}(\mathfrak{g})$ can be (locally) represented as $f=F\left(f_{1}, \ldots, f_{r}\right)$ for some smooth function $F$ and the functions $f_{1}, \ldots, f_{r}$ are functionally independent (in some neighborhood).

Let us use relations of item 3.8 , chapter 2 , in order to find first prolongations of fields (1). We have

$$
\left\{\begin{array}{l}
X_{1}^{(1)}=\frac{\partial}{\partial p_{0}} \\
X_{2}^{(1)}=p_{0} \frac{\partial}{\partial p_{0}}+p_{1} \frac{\partial}{\partial p_{1}}, \\
X_{3}^{(1)}=p_{0}^{2} \frac{\partial}{\partial p_{0}}+2 p_{1} p_{0} \frac{\partial}{\partial p_{1}} .
\end{array} .\right.
$$

If $f=f\left(t, p_{0}, p_{1}\right)$ is a differential invariant of $\mathfrak{s l}(2, \mathbb{R})$, then

$$
X_{1}^{(1)}(f)=X_{2}^{(1)}(f)=X_{3}^{(1)}(f)=0
$$

and therefore

$$
\frac{\partial f}{\partial p_{0}}=\frac{\partial f}{\partial p_{1}}=0
$$

Thus the function $f=t$ forms a basis of $I_{1}(\mathfrak{s l}(2, \mathbb{R}))$.

Further

$$
\left\{\begin{array}{l}
X_{1}^{(2)}=\frac{\partial}{\partial p_{0}} \\
X_{2}^{(2)}=p_{0} \frac{\partial}{\partial p_{0}}+p_{1} \frac{\partial}{\partial p_{1}}+p_{2} \frac{\partial}{\partial p_{2}} \\
X_{3}^{(2)}=p_{0}^{2} \frac{\partial}{\partial p_{0}}+2 p_{1} p_{0} \frac{\partial}{\partial p_{1}}+2\left(p_{2} p_{0}+p_{1}^{2}\right) \frac{\partial}{\partial p_{2}} .
\end{array}\right.
$$

It follows that $I_{2}(\mathfrak{s l}(2, \mathbb{R}))$ is also generated by the function $f=t$. 
For $k=3$ we have

$$
\left\{\begin{array}{l}
X_{1}^{(3)}=\frac{\partial}{\partial p_{0}} \\
X_{2}^{(3)}=p_{0} \frac{\partial}{\partial p_{0}}+p_{1} \frac{\partial}{\partial p_{1}}+p_{2} \frac{\partial}{\partial p_{2}}+p_{3} \frac{\partial}{\partial p_{3}} \\
X_{3}^{(3)}=p_{0}^{2} \frac{\partial}{\partial p_{0}}+2 p_{1} p_{0} \frac{\partial}{\partial p_{1}}+2\left(p_{2} p_{0}+p_{1}^{2}\right) \frac{\partial}{\partial p_{2}}+2\left(p_{0} p_{3}+3 p_{1} p_{2}\right) \frac{\partial}{\partial p_{3}}
\end{array}\right.
$$

Therefore, if $f\left(t, p_{0}, p_{1}, p_{2}, p_{3}\right) \in I_{3}(\mathfrak{s l}(2, \mathbb{R}))$, then

$$
\left\{\begin{array}{l}
\frac{\partial f}{\partial p_{0}}=0 \\
p_{1} \frac{\partial f}{\partial p_{1}}+p_{2} \frac{\partial f}{\partial p_{2}}+p_{3} \frac{\partial f}{\partial p_{3}}=0 \\
p_{1}^{2} \frac{\partial f}{\partial p_{2}}+3 p_{1} p_{2} \frac{\partial f}{\partial p_{3}}=0
\end{array}\right.
$$

From the first two equations it follows that

$$
f=g(t, x, y),
$$

where $x=\frac{p_{2}}{p_{1}}$ and $y=\frac{p_{3}}{p_{1}}$, and the last equation implies that the function $g(t, x, y)$ satisfies the following equation:

$$
\frac{\partial g}{\partial x}+3 x \frac{\partial g}{\partial y}=0
$$

Therefore

$$
g=g\left(t, y-\frac{3}{2} x^{2}\right)
$$

Thus, the functions

$$
f_{1}=t, f_{2}=y-\frac{3}{2} x^{2}=\frac{p_{3}}{p_{1}}-\frac{3}{2}\left(\frac{p_{2}}{p_{1}}\right)^{2}
$$

form a basis of invariants of the third order.

The restriction of the differential invariant $f_{2}$ to the 3 -jet of a function $z=z(t)$

$$
\left(j_{3}(z)\right)^{*}\left(f_{2}\right)=\frac{z^{\prime \prime}}{z^{\prime}}-\frac{3}{2}\left(\frac{z^{\prime \prime}}{z^{\prime}}\right)^{2} .
$$

is called the Schwarz derivative or Schwarzian of $z(t)$.

The Lie algebra $\mathfrak{s l}(2, \mathbb{R})$ considered as an algebra of point symmetries can be realized in a different way:

$$
X_{1}=\frac{\partial}{\partial t}, X_{2}=t \frac{\partial}{\partial t}, X_{3}=t^{2} \frac{\partial}{\partial t} .
$$

This representation corresponds to the action of $\mathrm{SL}(2, \mathbb{R})$ by linear fractional transformations of the $t$-axis (cp. (2)). Proceeding as before, we see that the functions

$$
\hat{f}_{1}=p_{0}, \hat{f}_{2}=2 \frac{p_{3}}{p_{1}^{3}}-3 \frac{p_{2}^{2}}{p_{1}^{4}}
$$

form a basis of invariants of the third order. 
Example 2. Consider the space $J^{0}(\mathbb{R}, \mathbb{R})$ as the two-dimensional Euclidean plane with the standard metric. Let $\mathfrak{g}$ be the Lie algebra of the movement group of the plane. For a basis of $\mathfrak{g}$ choose the following vector fields:

$$
T=\frac{\partial}{\partial t}, U=\frac{\partial}{\partial p_{0}}, \Pi=t \frac{\partial}{\partial p_{0}}-p_{0} \frac{\partial}{\partial t},
$$

corresponding to translations along axes $t$ and $p_{0}$ and rotations, respectively. Their prolongations to the space $J^{1}(\mathbb{R}, \mathbb{R})$ have the form

$$
\begin{aligned}
T^{(1)} & =\frac{\partial}{\partial t} \\
U^{(1)} & =\frac{\partial}{\partial p_{0}} \\
\Pi^{(1)} & =-p_{0} \frac{\partial}{\partial t}+t \frac{\partial}{\partial p_{0}}+\left(1+p_{1}^{2}\right) \frac{\partial}{\partial p_{1}} .
\end{aligned}
$$

It follows that $\mathfrak{g}$ has no nontrivial differential invariants of the first order. All subsequent prolongations do not change the local form of the fields $T$ and $U$, whereas for the vector field $\Pi$ we have

$$
\Pi^{(2)}=-p_{0} \frac{\partial}{\partial t}+t \frac{\partial}{\partial p_{0}}+\left(1+p_{1}^{2}\right) \frac{\partial}{\partial p_{1}}+3 p_{1} p_{2} \frac{\partial}{\partial p_{2}}
$$

and

$$
\Pi^{(3)}=-p_{0} \frac{\partial}{\partial t}+t \frac{\partial}{\partial p_{0}}+\left(1+p_{1}^{2}\right) \frac{\partial}{\partial p_{1}}+3 p_{1} p_{2} \frac{\partial}{\partial p_{2}}+\left(4 p_{1} p_{3}+3 p_{2}^{2}\right) \frac{\partial}{\partial p_{3}} .
$$

Therefore, second-order differential invariants are functions $f=f\left(p_{1}, p_{2}\right)$ satisfying the equation

$$
\left(1+p_{1}^{2}\right) \frac{\partial f}{\partial p_{1}}+3 p_{1} p_{2} \frac{\partial f}{\partial p_{2}}=0 .
$$

The corresponding vector field has the form

$$
\left(1+p_{1}^{2}\right) \frac{\partial}{\partial p_{1}}+3 p_{1} p_{2} \frac{\partial}{\partial p_{2}}
$$

Translations along this field can be calculated in an explicit form, so that we obtain the following second-order differential invariant

$$
f_{1}=\frac{p_{2}}{\left(1+p_{1}^{2}\right)^{3 / 2}}
$$

Restriction of $f_{1}$ to the 2-jet of a function $z(t)$ gives the curvature of the curve $\left\{p_{0}=z(t)\right\} \subset J^{0}(\mathbb{R}, \mathbb{R})$. 
Similarly, solving the equation

$$
\left(1+p_{1}^{2}\right) \frac{\partial f}{\partial p_{1}}+3 p_{1} p_{2} \frac{\partial f}{\partial p_{2}}+\left(4 p_{1} p_{3}+3 p_{2}^{2}\right) \frac{\partial f}{\partial p_{3}}=0
$$

with respect to $f=f\left(p_{1}, p_{2}, p_{3}\right)$, we obtain the following third-order differential invariant:

$$
f_{2}=\frac{p_{3}\left(1+p_{1}^{2}\right)}{p_{2}^{2}}-3 p_{1}
$$

Thus, the Lie algebra $\mathfrak{g}$ of movements of the plane has two independent differential invariants of the third order: $f_{1}$ and $f_{2}$.

4.4. If $\operatorname{dim} M=1$, we can consider Lie algebras of contact vector fields on $J^{1}(\mathbb{R}, \mathbb{R})$, their prolongations and differential invariants.

Example. Consider the Lie algebra $\mathfrak{g}$ of contact vector fields on $J^{1}(\mathbb{R}, \mathbb{R})$ generated by two vector fields

$$
\begin{aligned}
& X_{\varphi_{1}}=p_{0} \frac{\partial}{\partial p_{0}}+p_{1} \frac{\partial}{\partial p_{1}} \\
& X_{\varphi_{2}}=-2 p_{1} \frac{\partial}{\partial t}-p_{1}^{2} \frac{\partial}{\partial p_{0}}
\end{aligned}
$$

with generating functions

$$
\varphi_{1}=p_{0} \text { and } \varphi_{2}=p_{1}^{2} .
$$

Let $f=f\left(t, p_{0}, p_{1}\right)$ be a differential invariant of the first order. Then the relation $X_{\varphi_{1}}(f)=0$ implies that

$$
f=h(t, x),
$$

where $x=\frac{p_{0}}{p_{1}}$. Substituting this expression in the equation $X_{\varphi_{2}}(f)=0$, we obtain

$$
2 \frac{\partial h}{\partial t}+\frac{\partial h}{\partial x}=0
$$

Thus, the function

$$
f_{1}=2 \frac{p_{0}}{p_{1}}-t
$$

forms a basis in the algebra of first order differential invariants.

Consider the prolongations of the vector fields $X_{\varphi_{1}}$ and $X_{\varphi_{2}}$ to the space of 2-jets:

$$
\begin{aligned}
& X_{\varphi_{1}}^{(2)}=p_{0} \frac{\partial}{\partial p_{0}}+p_{1} \frac{\partial}{\partial p_{1}}+p_{2} \frac{\partial}{\partial p_{2}} \\
& X_{\varphi_{2}}^{(2)}=-2 p_{1} \frac{\partial}{\partial t}-p_{1}^{2} \frac{\partial}{\partial p_{0}}+2 p_{2}^{2} \frac{\partial}{\partial p_{2}}
\end{aligned}
$$


Let $f=f\left(t, p_{0}, p_{1}, p_{2}\right)$ be a differential invariant of the second order. Then the relation $X_{\varphi_{1}}^{(2)}(f)=0$ implies that

$$
f=h(t, x, y),
$$

where $x=\frac{p_{0}}{p_{1}}, y=\frac{p_{1}}{p_{2}}$, and $h$ is an arbitrary function. In terms of these coordinates, the relation $X_{\varphi_{2}}^{(2)}(f)=0$ takes the form:

$$
2 \frac{\partial h}{\partial t}+\frac{\partial h}{\partial x}+2 \frac{\partial h}{\partial y}=0
$$

Therefore, the functions

$$
f_{1}=2 \frac{p_{0}}{p_{1}}-t, f_{2}=2 \frac{p_{0}}{p_{1}}-\frac{p_{1}}{p_{2}}
$$

form a basis in the algebra of second-order differential invariants.

4.5. The following result, which apparently was first pointed out by Tresse, allows to construct new differential invariants starting from already-known ones.

Definition. Let $f, g \in C^{\infty}\left(J^{k}\left(\mathbb{R}, \mathbb{R}^{n}\right)\right)$ be arbitrary functions. Their Tresse derivative is the quotient

$$
\frac{d f}{d g} \stackrel{\text { def }}{=} \frac{d f}{d t} / \frac{d g}{d t}
$$

where

$$
\frac{d}{d t}=\frac{\partial}{\partial t}+\sum_{i, j} p_{j+1}^{i} \frac{\partial}{\partial p_{j}^{i}}
$$

Theorem. Let $f, g \in C^{\infty}\left(J^{k}\left(\mathbb{R}, \mathbb{R}^{n}\right)\right)$ be $k$-th order differential invariants of a Lie algebra $\mathfrak{g}$. Then their Tresse derivative is also a differential invariant of $\mathfrak{g}$.

Let us first prove the following lemma:

\section{Lemma.}

(1) Let $X$ be a vector field defined on the manifold $\mathbb{R} \times \mathbb{R}^{n}$ and $X^{(k)}$ its $k$-th prolongation. If

$$
X=A\left(t, p_{0}\right) \frac{\partial}{\partial t}+\sum_{i} B^{i}\left(t, p_{0}\right) \frac{\partial}{\partial p_{0}^{i}}
$$

then

$$
-X^{(k+1)} \circ \frac{d}{d t}+\frac{d}{d t} \circ X^{(k)}=\frac{d A}{d t} \frac{d}{d t}
$$

(2) Let $X=X_{f}$ be a contact vector field defined on the space $J^{1}(\mathbb{R}, \mathbb{R})$. Then

$$
X^{(k+1)} \circ \frac{d}{d t}-\frac{d}{d t} \circ X^{(k)}=\frac{d}{d t}\left(\frac{\partial f}{\partial p_{1}}\right) \frac{d}{d t}
$$


Proof of the lemma. Since proofs of (13) and (14) are similar to each other, we shall prove the first of the relations. We shall make use of relations 3.8.(18) and 3.10.(23) of chapter 2 .

Note that since operators in both sides of (13) are linear, it is sufficient to show that relation (13) holds for coordinate functions. We have

$$
\begin{gathered}
\left(X^{(k+1)} \circ \frac{d}{d t}-\frac{d}{d t} \circ X^{(k)}\right)(t)=-\frac{d A}{d t} \\
\left(X^{(k+1)} \circ \frac{d}{d t}-\frac{d}{d t} \circ X^{(k)}\right)\left(p_{j}^{i}\right)=B_{j+1}^{i}-\frac{d}{d t} B_{j}^{i}=-p_{j+1}^{i} \frac{d A}{d t} .
\end{gathered}
$$

Proof of the theorem. Let $X \in \mathfrak{g}$. Since $X^{(k)}(f)=X^{(k)}(g)=0$, we have

$$
\begin{aligned}
X^{(k+1)}\left(\frac{d f}{d t} / \frac{d g}{d t}\right)=\left(X^{(k+1)}\left(\frac{d f}{d t}\right)\right. & \left.\frac{d g}{d t} \frac{d f}{d t} X^{(k+1)}\left(\frac{d g}{d t}\right)\right) /\left(\frac{d g}{d t}\right)^{2}= \\
& =\left(\frac{d A}{d t} \frac{d f}{d t} \frac{d g}{d t}-\frac{d f}{d t} \frac{d A}{d t} \frac{d g}{d t}\right) /\left(\frac{d g}{d t}\right)^{2}=0
\end{aligned}
$$

Corollary. Suppose that functions $g, f_{1}, \ldots, f_{n} \in C^{\infty}\left(J^{k}\left(\mathbb{R}, \mathbb{R}^{n}\right)\right)$ form a basis of the space of $k$-th order differential invariants and the following conditions hold:

$$
\frac{\partial g}{\partial p_{k}^{i}}=0, i=1, \ldots, n
$$

and

$$
\operatorname{det}\left\|\frac{\partial f_{i}}{\partial p_{k}^{j}}\right\| \neq 0
$$

Then the Tresse derivatives

$$
\frac{d f_{i}}{d g}, \frac{d^{2} f_{i}}{d g^{2}}, \ldots, i=1, \ldots, n,
$$

generate all differential invariants of $\mathfrak{g}$.

Proof of the corollary. It is sufficient to note that

$$
\frac{d f_{i}}{d g}=\frac{\sum p_{k+1}^{j} \frac{\partial f_{i}}{\partial p_{k}^{j}}+\ldots}{\frac{d g}{d t}}=\sum p_{k+1}^{j}\left(\frac{\partial f_{i}}{\partial p_{k}^{j}} / \frac{d g}{d t}\right)+\ldots
$$

where dots denote functions dependent on $p_{j}^{i}(j \leqslant k)$. Therefore, the functions $\frac{\partial f_{i}}{\partial g}$ form a basis in the algebra of $(k+1)$-th order differential invariants and also satisfy condition (15). Induction on $k$ concludes the proof. 
4.6. Let us apply the results of the previous item to finding differential invariants of the algebras considered in examples 4.3 and 4.4 .

A) The algebra $\mathfrak{s l}(2, \mathbb{R})$.

1) For invariants 4.3.(7)

$$
f_{1}=t, f_{2}=\frac{p_{3}}{p_{1}}-\frac{3}{2}\left(\frac{p_{2}}{p_{1}}\right)^{2}
$$

we have

$$
f_{3}=\frac{d f_{2}}{d f_{1}}=\frac{d f_{2}}{d t}=\frac{p_{4}}{p_{1}}-4 \frac{p_{2} p_{3}}{p_{1}^{2}}+3\left(\frac{p_{2}}{p_{1}}\right)^{3} .
$$

2) For invariants 4.3.( $\left.7^{\prime}\right)$

$$
\hat{f}_{1}=p_{0}, \hat{f}_{2}=2 \frac{p_{3}}{p}-3\left(\frac{p_{2}}{p_{1}^{2}}\right)^{2}
$$

we have

$$
\hat{f}_{3}=\frac{d \hat{f}_{2}}{d \hat{f}_{1}}=\frac{1}{p_{1}} \frac{d \hat{f}_{2}}{d t}=2 \frac{p_{4}}{p_{1}^{4}}-12 \frac{p_{2} p_{3}}{p_{1}^{5}}+12\left(\frac{p_{2}}{p_{1}^{2}}\right)^{3} .
$$

Thus, $\mathfrak{s l}(2, \mathbb{R})$ can be considered as an algebra of shuffling symmetries of equations

$$
F\left(f_{1}, f_{2}, f_{3}\right)=0 \text { and } G\left(\hat{f}_{1}, \hat{f}_{2}, \hat{f}_{3}\right)=0
$$

where $F$ and $G$ are arbitrary functions.

B) The algebra of movements of the plane (see 4.3.(8)).

For the invariants

$$
f_{1}=\frac{p_{2}}{\left(1+p_{1}^{2}\right)^{3 / 2}}, f_{2}=\frac{p_{3}\left(1+p_{1}^{2}\right)}{p_{2}^{2}}-3 p_{1}
$$

we have

$$
f_{3}=\frac{d f_{2}}{d f_{1}}=\frac{p_{4} p_{2}^{-2}\left(1+p_{1}^{2}\right)-2 p_{3}^{2} p_{2}^{-3}\left(1+p_{1}^{2}\right)+2 p_{1} p_{3} p_{2}^{-1}-3 p_{2}}{\left(p_{3}+p_{1}^{2} p_{3}-3 p_{1} p_{2}^{2}\right)\left(1+p_{1}^{2}\right)^{5 / 2}}
$$

Therefore, the algebra of movements of the plane can be considered as an algebra of shuffling symmetries of the equation

$$
F\left(f_{1}, f_{2}, f_{3}\right)=0
$$

where $F$ is an arbitrary function.

C)The commutative algebra $\mathfrak{g}$ generated by the contact vector fields $X_{\varphi_{1}}$ and $X_{\varphi_{2}}$, where $\varphi_{1}=p_{0}$ and $\varphi_{2}=p_{1}^{2}$. 
For the invariants

$$
f_{1}=2 \frac{p_{0}}{p_{1}}-t, f_{2}=2 \frac{p_{0}}{p_{1}}-\frac{p_{1}}{p_{2}}
$$

we have

$$
f_{3}=\frac{d f_{2}}{d f_{1}}=\frac{p_{1}^{2} p_{2}^{2}-2 p_{0} p_{2}^{3}+p_{1}^{3} p_{3}}{p_{2}^{2}\left(p_{1}^{2}-2 p_{0} p_{2}\right)} .
$$

Thus, $\mathfrak{g}$ can be considered as an algebra of contact symmetries for equations of the form:

$$
F\left(f_{1}, f_{2}, f_{3}\right)=0
$$

4.7. Example. [Halfan]. Consider the group of projective transformations of the plane $(t, u), u=p_{0}$. This group coincides with the factor group of $\mathrm{SL}(3, \mathbb{R})$ with respect to the center $\mathbb{Z}_{2}=\{1,-1\}$.

Each matrix $A=\left\|a_{i j}\right\| \in \mathrm{SL}(3, \mathbb{R})$ determines a projective transformation of the plane:

$$
A:(t, u) \mapsto(T, U),
$$

where

$$
T=\frac{a_{11} t+a_{12} u+a_{13}}{a_{31} t+a_{32} u+a_{33}}, U=\frac{a_{21} t+a_{22} u+a_{23}}{a_{31} t+a_{32} u+a_{33}} .
$$

In this case the Lie algebra $\mathfrak{s l}(3, \mathbb{R})$ can be considered as the algebra of vector fields on the plane with the following basis:

$$
\begin{gathered}
A_{1}=\frac{\partial}{\partial t}, A_{2}=\frac{\partial}{\partial u}, A_{3}=t \frac{\partial}{\partial t}, A_{4}=u \frac{\partial}{\partial t} \\
A_{5}=t \frac{\partial}{\partial u}, A_{6}=u \frac{\partial}{\partial t}, A_{7}=t\left(t \frac{\partial}{\partial t}+u \frac{\partial}{\partial u}\right), A_{8}=u\left(t \frac{\partial}{\partial t}+u \frac{\partial}{\partial u}\right) .
\end{gathered}
$$

Direct calculation shows that prolongations of these fields are independent up to 6-jets. It follows that first nontrivial differential invariants of the projective group are of the seventh order. Prior to the seventh order, the group has relative differential invariants, i.e. functions $f\left(t, p_{0}, \ldots, p_{k}\right)$ such that $\left(A^{(k)}\right)^{*}(f)=\lambda_{A} f$ for all $A \in \operatorname{SL}(3, \mathbb{R}), \lambda_{A}=$ $\lambda_{A}\left(t, p_{0}, \ldots, p_{k}\right)$.

The simplest relative differential invariants can be found from geometrical considerations.

Firstly, the projective group preserves the class of straight lines on the plane $(t, u)$. Therefore, this group is a symmetry group of the equation $p_{2}=0$, so that

$$
f_{1}=p_{2}
$$

is a relative invariant.

Secondly, the projective group preserves the class of quadrics on the plane. Equations of all quadrics on the plane can be reduced to the form $u=y(t)$, where

$$
y=a_{1} t+a_{2}+\sqrt{b_{1} t^{2}+2 b_{2} t+b_{3}} .
$$


Differentiating this expression two times, we obtain

$$
y^{\prime \prime}=\frac{b_{1} b_{3}-b_{2}^{2}}{\left(b_{1} t^{2}+2 b_{2} t+b_{3}\right)^{3 / 2}}
$$

or

$$
\left(y^{\prime \prime}\right)^{-2 / 3}=\frac{b_{1} t^{2}+2 b_{2} t+b_{3}}{\left(b_{1} b_{3}-b_{2}^{2}\right)^{2 / 3}} \text {. }
$$

It follows that

$$
\frac{d^{3}}{d t^{3}}\left(y^{\prime \prime}\right)^{-2 / 3}=0
$$

Differentiating this expression, we obtain the following relative invariant of the fifth order:

$$
f_{2}=p_{2}^{2} p_{5}-3 p_{2} p_{3} p_{4}+2 p_{3}^{3}
$$

Halfan gave two more relative invariants of the seventh order:

$$
f_{3}=\left|\begin{array}{ccccc}
p_{3} & p_{4} & p_{5} & p_{6} & p_{7} \\
p_{2} & p_{3} & p_{4} & p_{5} & p_{6} \\
-p_{2}^{2} & 0 & p_{3}^{2} & 2 p_{3} p_{4} & 2 p_{3} p_{5}+p_{4}^{2} \\
0 & p_{2}^{2} & 2 p_{2} p_{3} & 2 p_{2} p_{4}+p_{3}^{2} & 2 p_{2} p_{5}+2 p_{3} p_{4} \\
0 & 0 & p_{2}^{2} & 3 p_{2} p_{3} & 3 p_{3}^{2}+3 p_{2} p_{4}
\end{array}\right|
$$

and

$$
f_{4}=\frac{256 f_{2}^{3}-27 f_{2}^{8}}{p_{2}^{4}} .
$$

The first differential invariant of the seventh order can be expressed by means of relative differential invariants:

$$
f_{5}=f_{3}^{3} f_{2}^{-8}
$$

The group $\mathrm{SL}(3, \mathbb{R})$ can be considered as a symmetry group of any of the equations $f_{i}=0, i=1, \ldots, 4$, and $f_{5}=$ const.

Let us indicate geometrical meaning of solutions of these equations. We have already done it for the equations $f_{1}=0$ and $f_{2}=0$. The equations $f_{3}=0$ and $f_{4}=0$ describe the class of so-called $W$-curves. The equation $f_{3}=0$ describes curves on the plane that, by means of projective transformations, can be turned into the logarithmic spiral that makes the constant angle $\pi / 6$ with the radius vector. The equation $f_{4}=0$ describes curves that are projectively equivalent to curves of the form $y^{2}=c t^{3}$. The equation $f_{5}=$ const also describes $W$-curves, i.e. curves projectively equivalent to curves of the form $y^{a} t^{b}=$ const. 


\section{§5. LAGRANGE INTEGRALS AND SUPERSYMMETRIES}

In this section we shall consider various ways of obtaining integrals from linear symmetries with the use of the Lagrange formula. We shall restrict ourselves to the case of self-adjoint or anti-self-adjoint matrix differential operators

$$
M=A_{0}(t) \partial^{n}+\cdots+A_{n}(t) .
$$

Here $A_{j}$ are functions of $\mathbb{R}$ into the vector space $\operatorname{Hom}\left(V, V^{*}\right)$ and $A_{0}(t)$ is non-singular.

5.1. Let $E=E(M)$ be the solution space of the homogeneous differential equation $M(h)=0$. Now if $M$ is self-adjoint, then from the Lagrange formula it follows that

$$
\partial P_{M}\left(h_{1}, h_{2}\right)=0 \Rightarrow P_{M}\left(h_{1}, h_{2}\right)=\text { const } \in \mathbb{R}
$$

for any two solutions $h_{1}, h_{2} \in E$. In particular, $P_{M}\left(h_{1}, \cdot\right)$ is a first integral for an arbitrary solution $h_{1}$.

Thus, the mapping

$$
\begin{gathered}
P_{M}: E(M) \times E(M) \rightarrow \mathbb{R} \\
\left(h_{1}, h_{2}\right) \mapsto P_{M}\left(h_{1}, h_{2}\right)
\end{gathered}
$$

determines a bilinear form on the solution space. This form is symmetric if $M$ is anti-self-adjoint and skew-symmetric if $M$ is self-adjoint.

Let us show that this bilinear form is non-singular. Indeed, suppose that a solution $h_{1} \in E(M), h_{1} \neq 0$, belongs to the kernel of the form. Then

$$
P_{M}\left(h_{1}, h\right)=0
$$

for all solutions $h \in E(M)$. Let $t_{0} \in \mathbb{R}$ be a point such that $A_{0}\left(t_{0}\right) \cdot h_{1}\left(t_{0}\right) \neq 0$ and $h(t)$ a solution such that

$$
h\left(t_{0}\right)=h^{(1)}\left(t_{0}\right)=\cdots=h^{(n-2)}\left(t_{0}\right)=0
$$

and

$$
\left\langle h_{1}\left(t_{0}\right), A_{0}^{t}\left(t_{0}\right) h^{(n-1)}\left(t_{0}\right)\right\rangle \neq 0 .
$$

Then the Lagrange formula implies that

$$
P_{M}\left(h, h_{1}\right)\left(t_{0}\right)=\left\langle h_{1}\left(t_{0}\right), A_{0}^{t}\left(t_{0}\right) h^{(n-1)}\left(t_{0}\right)\right\rangle \neq 0
$$

which contradicts the choice of the function $h_{1}$.

Definition. Let $M$ be a (anti-)self-adjoint operator. The bilinear form $P_{M}\left(h_{1}, h_{2}\right)$ on the space $E(M)$ is called the Lagrange form.

Summarizing the preceding, we obtain the following result: 
Theorem. The Lagrange form on the space $E(M)$ determines

(i) a symplectic structure if $M$ is self-adjoint,

(ii) a pseudo-Euclidean structure if $M$ is anti-self-adjoint.

5.2. Let us consider some examples. Let $V=\mathbb{R}$ and if $\left\{h_{1}, \ldots, h_{k}\right\}$ is a basis of the space $E(M)$, then by $\left\{h_{1}^{*}, \ldots, h_{k}^{*}\right\}$ denote the dual basis of the dual space $(E(M))^{*}$.

1) Suppose $M=\partial$. Then the space $E(M)$ is one-dimensional and the function $h_{1}=1$ forms a basis of $E(M)$. In addition, $P_{M}\left(h_{1}, h_{1}\right)=1$ and therefore

$$
P_{M}=\left(h_{1}^{*}\right)^{2} .
$$

2) Suppose $M=\partial^{2}$. Then the space $E(M)$ is two-dimensional and the functions $h_{1}=1$ and $h_{2}=t$ form a basis of $E(M)$. In addition, $P_{M}\left(h_{1}, h_{2}\right)=-1$, so that

$$
P_{M}=h_{2}^{*} \wedge h_{1}^{*}
$$

3) Suppose $M=\partial^{3}$. The space $E(M)$ is three-dimensional and the functions $h_{1}=1, h_{2}=t, h_{3}=\frac{1}{2} t^{2}$ can be chosen as a basis of $E(M)$. In this case

$$
P_{M}=-\left(h_{2}^{*}\right)^{2}+h_{1}^{*} \cdot h_{3}^{*} .
$$

5.3. Actually, it is not necessary to know the explicit description of the space $E(M)$ in order to calculate the form $P_{M}$. For example, it can be done in the following way. A solution $h(t)$ of the differential equation $M(h)=0$ is completely determined by initial conditions:

$$
h\left(t_{0}\right)=x_{0}, \ldots, h^{(n-1)}\left(t_{0}\right)=x_{n-1} .
$$

Thus, relations (1) give an isomorphism between $E(M)$ and $\mathbb{R}^{N}$, where $N=n \cdot \operatorname{dim} V$. In this case the Lagrange formula allows to calculate $P_{M}$ as a bilinear form on $\mathbb{R}^{N}$.

\section{Examples.}

1) Consider second-order differential equations $M(h)=0$, where

$$
M=m_{0}(t) \partial^{2}+m_{1}(t) \partial+m_{2}(t) .
$$

Then

$$
P_{M}(X, Y)=m_{0}\left(t_{0}\right)\left(x_{1} y_{0}-y_{1} x_{0}\right)+\left(m_{1}\left(t_{0}\right)-m_{0}^{\prime}\left(t_{0}\right)\right) x_{0} y_{0},
$$

where $X=\left(x_{0}, x_{1}\right)$ and $Y=\left(y_{0}, y_{1}\right)$.

2) For third-order differential equations $M(h)=0$, where

$$
M=m_{0}(t) \partial^{3}+m_{1}(t) \partial^{2}+m_{2}(t) \partial+m_{3}(t)
$$

we obtain

$$
\begin{aligned}
P_{M}(X, Y)=m_{0}\left(t_{0}\right)\left(x_{2} y_{0}+\right. & \left.y_{2} x_{0}-x_{1} y_{1}\right)+m_{1}\left(t_{0}\right)\left(x_{1} y_{0}-y_{1} x_{0}\right)+ \\
& +2 m_{0}^{\prime}\left(t_{0}\right) x_{0} y_{1}+\left(m_{2}\left(t_{0}\right)-m_{1}^{\prime}\left(t_{0}\right)+m_{0}^{\prime \prime}\left(t_{0}\right)\right) x_{0} y_{0}
\end{aligned}
$$


where $X=\left(x_{0}, x_{1}, x_{2}\right)$ and $Y=\left(y_{0}, y_{1}, y_{2}\right)$.

5.4. Let us digress into linear algebra for a while.

Let $E(=E(M))$ be a finite-dimensional pseudo-Euclidean space with a structure form $P\left(=P_{M}\right)$. It is known that to each bilinear form $Q$ on $E$ we can assign a linear operator $\widehat{Q}: E \rightarrow E$ such that

$$
Q(X, Y)=P(\widehat{Q} X, Y)
$$

for all $X, Y \in E$. By $*$ denote the operator of adjunction on $\operatorname{End}(E)$ determined by the form $P$ :

$$
P(A X, Y)=P\left(X, A^{*} Y\right)
$$

for all $X, Y \in E$.

Let $\Lambda^{2}\left(E^{*}\right)$ denote the space of all skew-symmetric bilinear forms on $E$ and $S^{2}\left(E^{*}\right)$ the space of all symmetric bilinear forms on $E$.

It can be easily checked that the following statement is true:

Proposition. A bilinear form $Q$ is either symmetric or skew-symmetric depending on whether the operator $\widehat{Q}$ is self-adjoint or anti-self-adjoint with respect to the involution $*$ :

$$
\begin{aligned}
& Q \in \Lambda^{2}\left(E^{*}\right) \Leftrightarrow \widehat{Q}^{*}+\widehat{Q}=0, \\
& Q \in \mathrm{S}^{2}\left(E^{*}\right) \Leftrightarrow \widehat{Q}^{*}-\widehat{Q}=0 .
\end{aligned}
$$

5.5. Now note that if $A$ and $B$ are self-adjoint operators, then so is their anticommutator

$$
\{A, B\}=\frac{1}{2}(A B+B A) .
$$

We shall make use of this observation in order to define an operation on the space $\mathrm{S}^{2}\left(E^{*}\right)$. If $Q_{1}, Q_{2} \in \mathrm{S}^{2}\left(E^{*}\right)$, then by $\left\{Q_{1}, Q_{2}\right\} \in \mathrm{S}^{2}\left(E^{*}\right)$ denote the symmetric bilinear form corresponding to the operator $\left\{\widehat{Q}_{1}, \widehat{Q}_{2}\right\}$, so that

$$
2\left\{Q_{1}, Q_{2}\right\}(X, Y)=Q_{1}\left(X, \widehat{Q}_{2} Y\right)+Q_{2}\left(X, \widehat{Q}_{1} Y\right) .
$$

It is obvious that the following statement is true:

Proposition. Anticommutation $Q_{1}, Q_{2} \mapsto\left\{Q_{1}, Q_{2}\right\}$ turns the space $\mathrm{S}^{2}\left(E^{*}\right)$ of symmetric bilinear forms on the pseudo-Euclidean space $E$ into a Jordan algebra with identity. The structure form $P$ is the identity of the algebra.

5.6. If operators $A$ and $B$ are anti-self-adjoint, then so is their commutator

$$
[A, B]=A B-B A \text {. }
$$

Using the isomorphism mentioned in 5.4, we can extend this operation to the space $\Lambda^{2}\left(E^{*}\right)$ of skew-symmetric bilinear forms: if $\alpha, \beta \in \Lambda^{2}\left(E^{*}\right)$, then by $[\alpha, \beta]$ denote the skew-symmetric bilinear form corresponding to the operator $[\hat{\alpha}, \hat{\beta}]$. Then

$$
[\alpha, \beta](X, Y)=\alpha(X, \hat{\beta} Y)+\beta(X, \hat{\alpha} Y) .
$$


Proposition. Commutation $\alpha, \beta \mapsto[\alpha, \beta]$ turns the space $\Lambda^{2}\left(E^{*}\right)$ into a Lie algebra isomorphic to $\mathfrak{s o}(P)$.

Proof. By definition, the Lie algebra $\mathfrak{s o}(P)$ consists of all linear operators $A: E \rightarrow E$ such that

$$
P(A X, Y)+P(X, A Y)=0
$$

for all $X, Y \in E$; in other words

$$
\mathfrak{s o}(P)=\left\{A: E \rightarrow E \mid A^{*}=-A\right\}
$$

Therefore,

$$
Q \in \Lambda^{2}\left(E^{*}\right) \Longleftrightarrow \widehat{Q} \in \mathfrak{s o}(P) .
$$

5.7. If an operator $A$ is anti-self-adjoint and $B$ is a self-adjoint operator, then their commutator $[A, B]$ is self-adjoint. Therefore, as before, we can define the commutator of a skew-symmetric form $\alpha \in \Lambda^{2}\left(E^{*}\right)$ and a symmetric form $Q \in \mathrm{S}^{2}\left(E^{*}\right)$ as the symmetric bilinear form corresponding to the operator $[\hat{\alpha}, \widehat{Q}]$. Thus, we obtain an action of the Lie algebra $\Lambda^{2}\left(E^{*}\right)$ on the Jordan algebra $S^{2}\left(E^{*}\right)$.

Finally, note that if operators $A$ and $B$ are self-adjoint, then their commutator is anti-self-adjoint. Therefore, we can define the commutator $\left[Q_{1}, Q_{2}\right] \in \Lambda^{2}\left(E^{*}\right)$ for elements of $\mathrm{S}^{2}\left(E^{*}\right)$. Similarly, if $A$ and $B$ are anti-self-adjoint, then their anticommutator is self-adjoint. Therefore, we can define the anticommutator $\{\alpha, \beta\} \in \mathrm{S}^{2}\left(E^{*}\right)$ for elements of $\Lambda^{2}\left(E^{*}\right)$.

5.8. Now let $E(=E(M))$ be a finite-dimensional symplectic space with a structure form $\Omega\left(=P_{M}\right)$. The correspondence $Q \mapsto \widetilde{Q}$ defined by

$$
Q(X, Y)=\Omega(\widetilde{Q} X, Y)
$$

gives an isomorphism of the space of bilinear forms on $E$ onto the space of linear operators on $E$. Denote by \# the operation of adjunction on $\operatorname{End}(E)$ determined by the form $\Omega$ :

$$
\Omega(A X, Y)=\Omega\left(X, A^{\#} Y\right)
$$

for all $X, Y \in E$.

The following results are similar to those of the previous items:

\section{Proposition.}

(1) $Q \in \mathrm{S}^{2}\left(E^{*}\right) \Leftrightarrow \widetilde{Q}^{\#}+\widetilde{Q}=0$, $Q \in \Lambda^{2}\left(E^{*}\right) \Leftrightarrow \widetilde{Q}^{\#}-\widetilde{Q}=0$.

(2) The space $\mathrm{S}^{2}\left(E^{*}\right)$ forms a Lie algebra with respect to commutation of forms. This algebra is isomorphic to $\mathfrak{s p}(\Omega)$.

(3) The space $\Lambda^{2}\left(E^{*}\right)$ forms a Jordan algebra with identity with respect to anticommutation of forms. The structure form $\Omega$ is the identity of this algebra. 
(4) The operations of commutation and anticommutation determine the following mappings:

$$
\begin{aligned}
\text { commutation }- & \Lambda^{2}\left(E^{*}\right) \times \Lambda^{2}\left(E^{*}\right) \rightarrow S^{2}\left(E^{*}\right), \\
& S^{2}\left(E^{*}\right) \times \Lambda^{2}\left(E^{*}\right) \rightarrow \Lambda^{2}\left(E^{*}\right) ; \\
\text { anticommutation }-\quad & S^{2}\left(E^{*}\right) \times S^{2}\left(E^{*}\right) \rightarrow \Lambda^{2}\left(E^{*}\right) .
\end{aligned}
$$

5.9. As we know, linear symmetries determine Lagrange forms and, thereby, first integrals.

Let $L$ be a self-adjoint operator and $(\Delta, \bar{\Delta}) \in \Pi(L)$. If $(\Delta, \bar{\Delta}) \in \Pi_{0}(L)$, then the operator $M=L \circ \Delta$ is anti-self-adjoint. Consider the Lagrange form corresponding to $M$. We shall denote it by $Q_{\Delta}\left(=P_{L \circ \Delta}\right)$. By the Lagrange identity

$$
\langle(L \circ \Delta) u, v\rangle+\langle u,(L \circ \Delta) v\rangle=\partial Q_{\Delta}(u, v) .
$$

Since $L \circ \Delta=-\Delta^{t} \circ L$, we see that the form $Q_{\Delta}(u, v)$ remains constant on every element of the space $E(L)$ and, thereby, determines a quadric on this space. If $(\Delta, \bar{\Delta}) \in \Pi_{1}(L)$, then the operator $M=L \circ \Delta$ is self-adjoint and similar reasoning shows that $Q_{\Delta}(u, v)$ is a skew-symmetric form on the space $E(L)$.

Now suppose $L$ is anti-self-adjoint. If $(\Delta, \bar{\Delta}) \in \Pi_{0}(L)$, then the corresponding form $Q_{\Delta}$ is skew-symmetric, whereas the form $Q_{\Delta}$ corresponding to $(\Delta, \bar{\Delta}) \in \Pi_{1}(L)$ is a symmetric form on $E(L)$. We can obtain new bilinear forms and new first integrals on the solution space, using the results of previous items.

Theorem. 1) Let $L$ be a self-adjoint differential operator. Then the Lagrange form $P_{L}$ determines a symplectic structure on the solution space $E(L)$. The correspondences

$$
\begin{aligned}
& \Pi_{0}(L) \ni(\Delta, \bar{\Delta}) \mapsto Q_{\Delta}=P_{L \circ \Delta} \in S^{2}\left(E^{*}\right), \\
& \Pi_{1}(L) \ni(\Delta, \bar{\Delta}) \mapsto Q_{\Delta}=P_{L \circ \Delta} \in \Lambda^{2}\left(E^{*}\right)
\end{aligned}
$$

give homomorphisms of the Lie algebra $\Pi_{0}(L)$ and the Jordan algebra $\Pi_{1}(L)$ into the corresponding algebras of forms on $E(L)$.

2) Let $L$ be an anti-self-adjoint differential operator. Then the Lagrange form $P_{L}$ determines a pseudo-Euclidean structure on the solution space $E(L)$. The correspondences

$$
\begin{aligned}
& \Pi_{0}(L) \ni(\Delta, \bar{\Delta}) \mapsto Q_{\Delta}=P_{L \circ \Delta} \in \Lambda^{2}\left(E^{*}\right), \\
& \Pi_{1}(L) \ni(\Delta, \bar{\Delta}) \mapsto Q_{\Delta}=P_{L \circ \Delta} \in S^{2}\left(E^{*}\right)
\end{aligned}
$$

give homomorphisms of the Lie algebra $\Pi_{0}(L)$ and the Jordan algebra $\Pi_{1}(L)$ into the corresponding algebras of forms on $E(L)$.

Remark. The isomorphism

$$
\varphi_{t}: E(L) \ni h \mapsto\left(h(t), h^{\prime}(t), \ldots, h^{(k-1)}(t)\right) \in J_{t}^{k-1}
$$


allows to consider every fundamental system of solutions of the equation $L h=0$ as a flow of symplectic transformations of $\mathbb{R}^{m}$ if $L$ is self-adjoint or pseudo-Euclidean isometries of $\mathbb{R}^{m}$ if $L$ is anti-self-adjoint.

5.10. In conclusion, consider application of Lagrange forms to nonlinear differential equations. For simplicity sake we restrict ourselves to the case of functions $y: \mathbb{R} \rightarrow \mathbb{R}$.

Consider a differential equation of the form:

$$
y^{(k)}=F\left(t, y, \ldots, y^{(k-1)}\right) .
$$

By $S$ denote the solution space of equation (11). Let us describe the space $\mathrm{T}_{h} S$ tangent to $S$ at a point $h \in S$. Let $h_{\tau}$ be a curve passing through the point $h, h_{\tau} \in S$, $h_{0}=h, \tau \in(-\varepsilon, \varepsilon) \subset \mathbb{R}$.

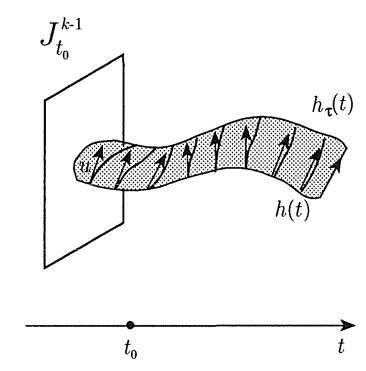

Fig. 20

The tangent vector

$$
u=\left.\frac{\partial h_{\tau}}{\partial \tau}\right|_{\tau=0}
$$

(see Fig. 20) can be identified with the function

$$
u(t)=\left.\frac{\partial h_{\tau}(t)}{\partial \tau}\right|_{\tau=0},
$$

which, by virtue of (11), satisfies the equation

$$
u^{(k)}=\sum_{i=0}^{k-1} \frac{\partial F}{\partial p_{i}}\left(t, h, \ldots, h^{(k-1)}\right) u^{(i)} .
$$

The operator

$$
L_{h}=\partial^{k}-\sum_{i=0}^{k-1} \frac{\partial F}{\partial p_{i}}(h) \partial^{i}
$$

is called the linearization of equation (11) on the solution $h$.

Thus,

$$
\mathrm{T}_{h} S=\left\{u \mid L_{h}(u)=0\right\} .
$$


Similarly, the cotangent space $\mathrm{T}_{h}^{*} S$ can be identified with the solution space of the adjoint equation:

$$
\mathrm{T}_{h}^{*} S=\left\{v \mid L_{h}^{t}(v)=0\right\} .
$$

Indeed, every solution $v$ of the adjoint equation $L_{h}^{t}(v)=0$ determines a linear function on the tangent space $\mathrm{T}_{h} S$ :

$$
\mathrm{T}_{h} S \ni u \mapsto P_{L_{h}}(u, v) \in \mathbb{R}
$$

The operator

$$
L=\mathcal{D}^{k}-\sum_{i=0}^{k-1} \frac{\partial F}{\partial p_{i}} \mathcal{D}^{i}
$$

where

$$
\mathcal{D}=\frac{\partial}{\partial t}+p_{1} \frac{\partial}{\partial p_{0}}+\cdots+p_{k-1} \frac{\partial}{\partial p_{k-2}}+F \frac{\partial}{\partial p_{k-1}}
$$

is called the Lie operator or the operator of total linearization.

By virtue of (14) a vector field on $S$ can be identified with a function

$$
\varphi\left(t, p_{0}, \ldots, p_{k-1}\right)
$$

satisfying the Lie equation:

$$
L \varphi=0 .
$$

Correspondingly, a differential 1-form on $S$ can be identified with a function

$$
\psi\left(t, p_{0}, \ldots, p_{k-1}\right)
$$

satisfying the adjoint equation:

$$
L^{t} \psi=0
$$

where

$$
L^{t}=(-1)^{k} \mathcal{D}^{k}-\sum_{i=0}^{k-1}(-1)^{i} \mathcal{D}^{i} \frac{\partial F}{\partial p_{i}} .
$$

Identifying the solution space with a fibre of the bundle $J^{k-1}(\mathbb{R}, \mathbb{R})$, we can represent covectors on $S$ as differential 1-forms of the form

$$
\omega=\sum_{i=0}^{k-1} a_{i}\left(t, p_{0}, \ldots, p_{k-1}\right) d p_{i} .
$$

In view of $\left(15^{\prime}\right)$ the differential form $\omega_{\psi}$ corresponding to a solution $\psi$ of equation (18) can be written as

$$
\omega_{\psi}=\sum_{r=0}^{k-1} \sum_{j=0}^{r-1}(-1)^{j} \mathcal{D}^{j}\left(\frac{\partial F}{\partial p_{r}} \psi\right) p_{r-j-1} .
$$


In addition, the restriction of the function

$$
P_{F}(\varphi, \psi)=\omega_{\psi}\left(X_{\varphi}\right)=\sum_{r=0}^{k-1} \sum_{j=0}^{r-1}(-1)^{j} \mathcal{D}^{j}\left(\frac{\partial F}{\partial p_{r}} \psi\right) \mathcal{D}^{r-j-1}(\varphi)
$$

to every solution $y=h(t)$ coincides with the Lagrange form $P_{L}\left(\varphi_{h}, \psi_{h}\right)$.

5.11. The results of the previous item can be easily extended to the more general case of equations of the form

$$
F\left(t, p_{0}, \ldots, p_{k}\right)=0
$$

where $\frac{\partial F}{\partial p_{k}} \neq 0$.

Let $\mathcal{E}$ be the submanifold given by (21). The description of vector fields and differential forms on the solution space $S$ given in the previous item still holds for equations (21) under assumption that $\mathcal{D}$ is the restriction of the operator $\frac{d}{d t}$ to $\mathcal{E}$ and that $L$ has the form:

$$
L=L_{F}=\frac{\partial F}{\partial p_{k}} \mathcal{D}^{k}+\frac{\partial F}{\partial p_{k-1}} \mathcal{D}^{k-1}+\cdots+\frac{\partial F}{\partial p_{0}}
$$

5.12. Definition. A differential operator

$$
\Delta=A_{n} \mathcal{D}^{n}+\cdots+A_{0}
$$

where $A_{n}, \ldots, A_{0}$ are functions on the manifold $\mathcal{E}$, is called a supersymmetry of the equation $\mathcal{E}$ if $\Delta$ is a linear symmetry of the Lie operator $L$.

The following statement is obviously true:

Theorem. Let $\Delta$ be a supersymmetry of the equation $\mathcal{E}$ and $\varphi$ a generating function of a shuffling symmetry of the equation. Then $\Delta(\varphi)$ is also a generating function of a symmetry of $\mathcal{E}$.

5.13. Using the description of linear symmetries given in $\S 5$ of the previous chapter, we obtain the following description of supersymmetries:

Proposition. A differential operator $\Delta$ is a supersymmetry of the equation $\mathcal{E}$ if and only if

$$
\bar{\Delta} \circ L=L \circ \Delta
$$

for some operator

$$
\bar{\Delta}=B_{n} \mathcal{D}^{n}+\cdots+B_{0}
$$

5.14. Definition. A differential equation $\mathcal{E}$ of form (21) is called self-adjoint (antiself-adjoint) if there exists a function $G=G\left(t, p_{0}, \ldots, p_{k}\right)$ such that the equation $\{G=0\}$ coincides with $\mathcal{E}$ and the operator $L_{G}$ is self-adjoint (anti-self-adjoint). 
5.15. Example. Let us describe self-adjoint second-order differential equations of the form:

$$
F\left(t, p_{0}, p_{1}, p_{2}\right)=0
$$

Here

and

$$
L=\frac{\partial F}{\partial p_{2}} \mathcal{D}^{2}+\frac{\partial F}{\partial p_{1}} \mathcal{D}+\frac{\partial F}{\partial p_{0}}
$$

$$
\begin{gathered}
L^{t}=\mathcal{D}^{2} \frac{\partial F}{\partial p_{2}}-\mathcal{D} \frac{\partial F}{\partial p_{1}}+\frac{\partial F}{\partial p_{0}}= \\
=\frac{\partial F}{\partial p_{2}} \mathcal{D}^{2}+\left(2 \mathcal{D}\left(\frac{\partial F}{\partial p_{2}}\right)-\frac{\partial F}{\partial p_{1}}\right) \mathcal{D}+\left(\mathcal{D}^{2}\left(\frac{\partial F}{\partial p_{2}}\right)-\mathcal{D}\left(\frac{\partial F}{\partial p_{1}}\right)+\frac{\partial F}{\partial p_{0}}\right) .
\end{gathered}
$$

Thus the equality $L=L^{t}$ holds if and only if

$$
\frac{\partial F}{\partial p_{1}}=\mathcal{D}\left(\frac{\partial F}{\partial p_{2}}\right)
$$

Let $F$ have the form $F=p_{2}+H\left(t, p_{0}, p_{1}\right)$ and $\varphi=\varphi\left(t, p_{0}, p_{1}\right)$. For the function $\varphi F$ condition (27) is equivalent to the following condition:

$$
\mathcal{D}(\ln |\varphi|)=\frac{\partial H}{\partial p_{1}}
$$

If $\frac{\partial H}{\partial p_{1}}=a(t)$, then $\varphi$ can be chosen as

$$
\varphi(t)=\exp \left(\int a(t) d t\right)
$$

Therefore, all differential equations of the form

$$
p_{2}+a(t) p_{1}+b\left(t, p_{0}\right)=0
$$

are self-adjoint.

5.16. By $\Pi(\mathcal{E})$ denote the set of all supersymmetries of the equation $\mathcal{E}$. Since the relation $L_{\varphi F}=\varphi L_{F}$ holds for the equation $\mathcal{E}=\{F=0\}$, we see that if $L_{F} \circ \Delta=$ $\bar{\Delta} \circ L_{F}$, then $L_{\varphi F} \circ \Delta=\bar{\Delta}_{\varphi} \circ L_{\varphi F}$, where $\bar{\Delta}_{\varphi}=\varphi \cdot \Delta \cdot \varphi^{-1}$. Thus, $\Pi(\mathcal{E})$ does not depend on a choice of the function $F$. If $\mathcal{E}$ is a self-adjoint (or anti-self-adjoint) equation, then the correspondence $\Delta \mapsto \Delta^{t}$ determines an involution of $\Pi(\mathcal{E})$ and therefore

$$
\Pi(\mathcal{E})=\Pi_{0}(\mathcal{E})+\Pi_{1}(\mathcal{E})
$$

where

$$
\begin{aligned}
\Pi_{0}(\mathcal{E}) & =\left\{\Delta \mid L_{F} \circ \Delta+\Delta^{t} L_{F}=0\right\}, \\
\Pi_{1}(\mathcal{E}) & =\left\{\Delta \mid L_{F} \circ \Delta-\Delta^{t} L_{F}=0\right\} .
\end{aligned}
$$

The constructions described in 5.8 allow to use supersymmetries in order to find new symmetries and integrals, starting from already-known ones. 


\section{CHAPTER IV}

\section{MODELS}

\section{$\S 1$. REDUCTION}

If a Lie algebra $\mathfrak{g}$ of shuffling symmetries of a completely integrable distribution $E$ is sufficiently large: $\operatorname{dim} \mathfrak{g} \geqslant \operatorname{codim} E$, then maximal integral manifolds of the distribution can be found by quadratures, i.e. by the use of the following two procedures: solving equations and calculating integrals. The latter can also be considered as solving a model differential equation

$$
y^{\prime}=f(t)
$$

for some function $f(t)$.

In this section we shall show how, given an arbitrary algebra of shuffling symmetries, to find the corresponding model differential equations, whose solutions should be known in order to integrate the initial equation.

1.1. Throughout the section we shall assume that a completely integrable distribution $E$ and algebra $\mathfrak{g}$ of shuffling symmetries satisfy conditions of $\S 2$, chapter 3 . In particular, $\operatorname{dim} \mathfrak{g}=\operatorname{codim} E$.

Let $\mathfrak{r} \subset \mathfrak{g}$ be an ideal in $\mathfrak{g}$ and $r=\operatorname{codim}_{\mathfrak{g}} \mathfrak{r}$. Suppose that $\left\{X_{1}, \ldots, X_{m}\right\}$ is a basis of $\mathfrak{g}$ such that the vectors $X_{r+1}, \ldots, X_{m}$ form a basis of $\mathfrak{r}$. As in $\S 2$ of chapter 3 , choose differential 1-forms $\omega_{1}, \ldots, \omega_{m}$ so that the distribution $E$ can be (maybe locally) given by these forms and

$$
\omega_{1}\left(X_{j}\right)=\delta_{i j}, i, j=1, \ldots, m
$$

Lemma. The distribution $E(\mathfrak{r})=\mathcal{F}\left(\omega_{1}, \ldots, \omega_{r}\right)$, given by the differential 1-forms $\omega_{1}, \ldots, \omega_{r}$, is completely integrable.

Proof. Since $\mathfrak{r}$ is an ideal in $\mathfrak{g}$, we see that all commutators of the form $\left[X_{i}, X_{j}\right]$, where $i=1, \ldots, m$ and $j=r+1, \ldots, n$, belong to $\mathfrak{r}$. Therefore,

$$
\left[X_{i}, X_{j}\right]=\sum_{s \geqslant r+1} C_{i j}^{s} X_{s}
$$

where

$$
C_{i j}^{s}=0
$$

under the condition that $j \geqslant r+1$ and $s \leqslant r$.

Using the Maurer-Cartan formula, we obtain

$$
d \omega_{l}=-1 / 2 \sum_{a, b \leqslant r} C_{a, b}^{l} \omega_{a} \wedge \omega_{b}
$$


if $l \leqslant r$. Therefore, the distribution $\mathcal{F}\left(\omega_{1}, \ldots, \omega_{r}\right)$ is completely integrable.

1.2. Note that the ideal $\mathfrak{r}$ is a symmetry algebra of the distribution $E(\mathfrak{r})$. Indeed, if $X \in \mathfrak{r}$, then $\omega_{1}(X)=\cdots=\omega_{r}(X)=0$ and from (2) it follows that

$$
L_{X}\left(\omega_{j}\right)=i_{X}\left(d \omega_{j}\right)=0
$$

for $j \leqslant r$.

Thus, vector fields $X \in \mathfrak{r}$ are characteristic for $E(\mathfrak{r})$.

The procedure of finding maximal integral manifolds of the distribution $E$ can be performed in two steps:

1) integration of the completely integrable distribution $E(\mathfrak{r})$;

2) integration of the completely integrable distribution $E_{L}$ obtained by restricting the distribution $E$ to maximal integral manifolds $L$ of the distributions $E(\mathfrak{r})$.

In this case the factor algebra $\mathfrak{g} / \mathfrak{r}$ and ideal $\mathfrak{r}$ can be chosen as algebras of shuffling symmetries for the distribution $E(\mathfrak{r})$ and $E_{L}$ respectively.

In particular, if $\mathfrak{r}$ is solvable, then integration of the distribution can be completed by quadratures.

Now let $\mathfrak{r}$ be the radical (i.e. maximal solvable ideal) of the Lie algebra $\mathfrak{g}$. Then the factor algebra $\mathfrak{g} / \mathfrak{r}$ is semisimple and thus we are led to integration of distributions with semisimple symmetry algebras. Further, every semisimple Lie algebra $\mathfrak{a}$ is a direct sum of simple Lie algebras, which are ideals in $\mathfrak{a}$. Therefore, in this case our problem reduces to integration of distributions with simple algebras of shuffling symmetries.

1.3. Let us turn to integration of distributions with simple symmetry algebras. We shall first consider the procedure of reduction.

\section{Definition.}

1) An action of a Lie algebra $\mathfrak{g}$ on a manifold $N$ is an injective homomorphism $\lambda: \mathfrak{g} \rightarrow \mathcal{D}(N)$ of $\mathfrak{g}$ into the Lie algebra of vector fields on $N$.

2) An action $\lambda$ of $\mathfrak{g}$ on $N$ is called transitive if the values of vector fields $\lambda(X), X \in \mathfrak{g}$, at each point $a \in N$ generate the whole of the space $\mathrm{T}_{a} N$.

\section{Examples.}

1) If $\operatorname{dim} \mathfrak{g}=1$, then an action $\lambda: \mathfrak{g} \rightarrow \mathcal{D}(N)$ is uniquely determined by the image $\lambda(l)$ of any nonzero vector $l \in \mathfrak{g}$ and therefore can be identified with a vector field on $N$.

2) Suppose $\mathfrak{g}=\mathfrak{s l}(2, \mathbb{R})$ and $N=\mathbb{R}^{1}$ is the projective line with homogeneous coordinates $\left[y_{1}: y_{2}\right]$. Consider the natural action of the group $S L(2, \mathbb{R})$ on the projective line

$$
A=\left[\begin{array}{ll}
a_{11} & a_{12} \\
a_{21} & a_{22}
\end{array}\right] \in S L(2, \mathbb{R}) \mapsto \Lambda(A): \mathbb{R} P^{1} \rightarrow \mathbb{R} P^{1},
$$

where

$$
\Lambda(A)\left(\left[y_{1}: y_{2}\right]\right)=\left[\left(a_{11} y_{1}+a_{12} y_{2}\right):\left(a_{21} y_{1}+a_{22} y_{2}\right)\right]
$$


The differential $\lambda$ of this action is an action of $\mathfrak{g}$ on $N$. Consider the following basis of $\mathfrak{s l}(2, \mathbb{R})$ :

$$
h=\left[\begin{array}{ll}
1 & 0 \\
0 & 1
\end{array}\right], a^{+}=\left[\begin{array}{ll}
0 & 1 \\
0 & 0
\end{array}\right], a^{-}=\left[\begin{array}{ll}
0 & 0 \\
1 & 0
\end{array}\right] .
$$

Then

$$
\begin{aligned}
\Lambda(\exp t h)\left(\left[y_{1}: y_{2}\right]\right) & =\left[e^{t} y_{1}: e^{-t} y_{2}\right], \\
\Lambda\left(\exp t a^{+}\right)\left(\left[y_{1}: y_{2}\right]\right) & =\left[\left(y_{1}+t y_{2}\right): y_{2}\right], \\
\Lambda\left(\exp t a^{-}\right)\left(\left[y_{1}: y_{2}\right]\right) & =\left[y_{1}:\left(y_{2}+t y_{1}\right)\right] .
\end{aligned}
$$

Let us identify $\mathbb{R}$ with the affine part of $\mathbb{R} P^{1}$ by means of the embedding

$$
\mathbb{R} \hookrightarrow \mathbb{R} P^{1}, s \mapsto[s: 1] .
$$

Then relations (4) take the form:

$$
\begin{aligned}
\Lambda(\exp t h) s & =e^{2 t} s \\
\Lambda\left(\exp t a^{+}\right) s & =s+t \\
\Lambda\left(\exp t a^{-}\right) s & =\frac{s}{1+t s} .
\end{aligned}
$$

The differential $\lambda$ has the form:

$$
\lambda\left(a_{j}\right)=\left.\frac{d}{d t} \Lambda\left(\exp t a_{j}\right)\right|_{t=0},
$$

so that

$$
\lambda(h)=2 s \frac{d}{d s}, \lambda\left(a^{+}\right)=\frac{d}{d s}, \lambda(h)=-s^{2} \frac{d}{d s} .
$$

3) This example is a direct generalization of the previous one. Suppose $\mathfrak{g}=$ $\mathfrak{s l}(n+1, \mathbb{R})$ and $N=\mathbb{R} P^{n}$ is the $n$-dimensional projective space with homogeneous coordinates $\left[y_{1}: \cdots: y_{n+1}\right]$. As before, consider the natural projective action of the group $S L(n+1, \mathbb{R})$ on the projective space $\mathbb{R} P^{n}$ :

$$
A=\left\|a_{i j}\right\| \in S L(n+1, \mathbb{R}) \mapsto \Lambda(A): \mathbb{R} P^{n} \rightarrow \mathbb{R} P^{n},
$$

where

$$
\Lambda(A)\left[y_{1}: \cdots: y_{n+1}\right]=\left[Y_{1}: \cdots: Y_{n+1}\right]
$$

and

$$
Y_{i}=\sum_{j=1}^{n+1} a_{i j} y_{j} .
$$

The differential $\lambda$ of this action is an action of $\mathfrak{g}$ on $N$.

For $i=1, \ldots, n$, by $h_{i}$ denote the diagonal matrix of order $n+1$ whose diagonal elements are all except for the $i$-th and $(i+1)$-th ones equal to 1 and -1 respectively. 
For $1 \leqslant i<j \leqslant n+1$ by $E_{i j}^{+}$denote the square matrix of order $n+1$ whose the only nonzero element is the element lying in the $i$-th row and the $j$-th column and equal to 1 . Put $E_{j i}^{-}=\left(E_{i j}^{+}\right)^{t}$. The matrices

$$
h_{1}, \ldots, h_{n} ; E_{i j}^{+}, E_{j i}^{-}, \quad 1 \leqslant i<j \leqslant n+1,
$$

form a basis of the Lie algebra $\mathfrak{s l}(n+1, \mathbb{R})$. We have

$$
\begin{aligned}
\Lambda\left(\exp t h_{i}\right)\left[y_{1}: \cdots: y_{n+1}\right] & =\left[y_{1}: \cdots: e^{t} y_{i}: e^{-t} y_{i+1}: \cdots: y_{n+1}\right] \\
\Lambda\left(\exp t E_{i j}^{+}\right)\left[y_{1}: \cdots: y_{n+1}\right] & =\left[y_{1}: \cdots: y_{i}+t y_{j}: \cdots: y_{j}: \cdots: y_{n+1}\right] \\
\Lambda\left(\exp E_{j i}^{-}\right)\left[y_{1}: \cdots: y_{n+1}\right] & =\left[y_{1}: \cdots: y_{i}: \cdots: y_{j}+t y_{i}: \cdots: y_{n+1}\right]
\end{aligned}
$$

Let us identify the arithmetic $n$-space $\mathbb{R}^{n}$ with the affine part of $\mathbb{R} P^{n}$ by means of the embedding

$$
\mathbb{R}^{n} \ni\left(x_{1}, \ldots, x_{n}\right) \mapsto\left(x_{1}: \cdots: x_{n}: 1\right) \in \mathbb{R} P^{n} .
$$

Then the differential $\lambda$ has the form:

$$
\begin{aligned}
& \lambda\left(h_{i}\right)=x_{i} \frac{\partial}{\partial x_{i}}-x_{i+1} \frac{\partial}{\partial x_{i}}, i=1, \ldots, n-1 ; \\
& \lambda\left(h_{n}\right)=x_{1} \frac{\partial}{\partial x_{1}}+\cdots+x_{n-1} \frac{\partial}{\partial x_{n-1}}+2 x_{n} \frac{\partial}{\partial x_{n}}, \\
& \lambda\left(E_{i j}^{+}\right)=x_{j} \frac{\partial}{\partial x_{i}}, 1 \leqslant i<j \leqslant n, \\
& \lambda\left(E_{i n+1}^{+}\right)=\frac{\partial}{\partial x_{i}}, i=1, \ldots, n, \\
& \lambda\left(E_{i j}^{-}\right)=x_{j} \frac{\partial}{\partial x_{i}}, 1 \leqslant j<i \leqslant n, \\
& \lambda\left(E_{n+1}^{-}\right)=-\sum_{r=1}^{n} x_{r} x_{i} \frac{\partial}{\partial x_{r}}, 1 \leqslant i \leqslant n .
\end{aligned}
$$

Let $A=\left\|a_{i j}\right\| \in \mathfrak{s l}(n+1, \mathbb{R})$. Since

$$
A=a_{11} h_{1}+\left(a_{11}+a_{12}\right) h_{2}+\cdots+\left(a_{11}+a_{n n}\right) h_{n}+\sum_{i<j} a_{i j} E_{i j}^{+}+\sum_{j<i} a_{i j} E_{i j}^{-},
$$

we obtain

$$
\begin{aligned}
& \lambda(A)=\sum_{i=1}^{n} a_{i i} x_{i} \frac{\partial}{\partial x_{i}}-a_{n+1} n+1 \sum_{i=1}^{n} x_{i} \frac{\partial}{\partial x_{i}}+\sum_{i<j \leqslant n} a_{i j} x_{i} \frac{\partial}{\partial x_{i}}+ \\
& \sum_{i=1}^{n} a_{i n+1} \frac{\partial}{\partial x_{i}}+\sum_{j<i \leqslant n} a_{i j} x_{i} \frac{\partial}{\partial x_{j}}-\left(\sum_{i=1}^{n} a_{n+1} x_{i}\right) \sum_{j=1}^{n} x_{i} \frac{\partial}{\partial x_{i}}
\end{aligned}
$$


or

$$
\begin{aligned}
\lambda(A)=\sum_{i, j=1}^{n} a_{i j} x_{i} \frac{\partial}{\partial x_{j}}- & \\
& \left(a_{n+1} n+1+\sum_{i=1}^{n} a_{i n+1} x_{i}\right) \sum_{j=1}^{n} x_{j} \frac{\partial}{\partial x_{j}}+\sum_{j=1}^{n} a_{n+1} \frac{\partial}{\partial x_{j}} .
\end{aligned}
$$

4) Reverting to the projective plane and its affine part $\mathbb{R}^{2}$, form (10) we see that if

$$
a=\left[\begin{array}{lll}
a_{11} & a_{12} & a_{13} \\
a_{21} & a_{22} & a_{23} \\
a_{31} & a_{32} & a_{33}
\end{array}\right] \text { and } a_{11}+a_{22}+a_{33}=0
$$

then the corresponding vector field on $\mathbb{R}^{2}$ has the form:

$$
\begin{aligned}
\lambda(A)=\left(a_{11} x_{1}+a_{21} x_{2}\right) \frac{\partial}{\partial x_{1}}+ & \left(a_{12} x_{1}+a_{22} x_{2}\right) \frac{\partial}{\partial x_{2}}+a_{31} \frac{\partial}{\partial x_{1}}+ \\
& a_{32} \frac{\partial}{\partial x_{2}}-\left(a_{33}+a_{13} x_{1}+a_{23} x_{2}\right)\left(x_{1} \frac{\partial}{\partial x_{1}}+x_{2} \frac{\partial}{\partial x_{2}}\right)
\end{aligned}
$$

or, after collecting terms,

$$
\begin{aligned}
& \lambda(A)=\left(a_{31}+\left(a_{11}-a_{33}\right) x_{1}\right.\left.+a_{21} x_{2}-a_{13} x_{1}^{2}-a_{23} x_{1} x_{2}\right) \frac{\partial}{\partial x_{1}}+ \\
&\left(a_{32}+a_{12} x_{1}+\left(a_{22}-a_{33}\right) x_{2}-a_{13} x_{1} x_{2}-a_{23} x_{2}^{2}\right) \frac{\partial}{\partial x_{2}} .
\end{aligned}
$$

1.4. Let $E$ be a completely integrable distribution on a manifold $M$ and $\mathfrak{g}$ an algebra of shuffling symmetries on $E$ (we shall identify elements of $\mathfrak{g}$ with vector fields on $M$ ) such that $\mathfrak{g}$ satisfies conditions of $\S 2$, chapter 3. Suppose $\lambda: \mathfrak{g} \rightarrow \mathcal{D}(N)$ is a transitive action of $\mathfrak{g}$ on some manifold $N$. Let us construct a new distribution $\widetilde{E}$ on the direct product $M \times N$ : for $a \in M$ and $b \in N$. Let $\widetilde{E}(a, b)$ be the direct sum of the subspace $E(a)$ and the subspace spanned by vectors of the form:

$$
X_{a}+\lambda(X)_{b}
$$

for all $X \in \mathfrak{g}$.

Lemma. The distribution $\widetilde{E}$ is completely integrable and

$$
\operatorname{codim} \widetilde{E}=\operatorname{dim} N \text {. }
$$


Proof. Let $Y_{1}, \ldots, Y_{n-m}$ be vector fields generating the distribution $E$ and let $\left\{X_{1}, \ldots, X_{m}\right\}$ be a basis of $\mathfrak{g}$. Then the vector fields

$$
Y_{1}, \ldots, Y_{n-m}, X_{1}+\lambda\left(X_{1}\right), \ldots, X_{m}+\lambda\left(X_{m}\right)
$$

can be chosen as a local basis of the distribution $\widetilde{E}$.

If is sufficient to note that

a) vector fields of the form $\left[Y_{i}, Y_{j}\right]$ belong to the distribution $E$ and therefore to the distribution $\widetilde{E}$;

b) since $X_{i}$ are symmetries of $E$, all vector fields $\left[X_{i}+\lambda\left(X_{i}\right), Y_{j}\right]=\left[X_{i}, Y_{j}\right]$ belong to $E$;

c) all vector fields $\left[X_{i}+\lambda\left(X_{i}\right), X_{j}+\lambda\left(X_{j}\right)\right]=\left[X_{i}, X_{j}\right]+\left[\lambda\left(X_{i}\right), \lambda\left(X_{j}\right)\right]=\left[X_{i}, X_{j}\right]+$ $\lambda\left(\left[X_{i}, X_{j}\right]\right)$ also belong to $\widetilde{E}$.

1.5. Let us describe the distribution $\widetilde{E}$ as a Pfaff system. Let $r=\operatorname{dim} N$ and let $\alpha_{1}, \ldots, \alpha_{r}$ be differential forms on $N$ that form a (local) basis in spaces cotangent to $N$. Consider the differential forms

$$
\begin{aligned}
& \theta_{1}=\alpha_{1}-\left\langle\alpha_{1}, \lambda\left(X_{1}\right)\right\rangle \omega_{1}-\cdots-\left\langle\alpha_{1}, \lambda\left(X_{m}\right)\right\rangle \omega_{m}, \\
& \quad \vdots \\
& \theta_{r}=\alpha_{r}-\left\langle\alpha_{r}, \lambda\left(X_{1}\right)\right\rangle \omega_{1}-\cdots-\left\langle\alpha_{r}, \lambda\left(X_{m}\right)\right\rangle \omega_{m},
\end{aligned}
$$

where $\left\{X_{1}, \ldots, X_{m}\right\}$ is a basis of the Lie algebra $\mathfrak{g}$ and $\omega_{1}, \ldots, \omega_{m}$ are forms generating $E$ such that $\omega_{i}\left(X_{j}\right)=\delta_{i j}$.

Lemma. The differential forms $\theta_{1}, \ldots, \theta_{r}$ determine the completely integrable distribution $\widetilde{E}$.

Proof. We shall employ the notation of the previous item. Then since for any vector field $Y_{j}, \alpha_{s}\left(Y_{j}\right)=0$ and $\omega_{i}\left(Y_{j}\right)=0, i=1, \ldots, n$, we have

$$
\theta_{s}\left(Y_{j}\right)=0, s=1, \ldots, r
$$

and

$$
\theta_{s}\left(X_{i}+\lambda\left(X_{i}\right)\right)=\alpha_{s}\left(\lambda\left(X_{i}\right)\right)-\left\langle\alpha_{s}, \lambda\left(X_{i}\right)\right\rangle \omega_{i}\left(X_{i}\right)=0 .
$$

It remains to note that the differential forms $\theta_{1}, \ldots, \theta_{r}$ are linearly independent and $r=\operatorname{codim} \widetilde{E}$.

1.6. Let us now consider maximal integral manifolds of the distribution $\widetilde{E}$. The projection of each manifold $L \subset M \times N$ of this sort into $M$ is a diffeomorphism of $L$ onto $M$. By construction of $\widetilde{E}$, the projection of a subspace $\widetilde{E}(a, b)$ into the tangent space $\mathrm{T}_{a} M$ is an isomorphism of $\widetilde{E}(a, b)$ onto $\mathrm{T}_{a} M$. Therefore (see Fig. 21), every maximal integral manifold $L$ determines the smooth mapping

$$
h: M \rightarrow N
$$


such that

$$
\left.L=L_{h}=\{(a, h(a)) \in M \times N)\right\}
$$

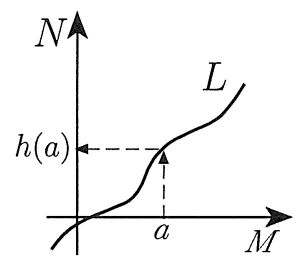

Fig. 21

The kernel of the differential $h_{*, a}: \mathrm{T}_{a} M \rightarrow \mathrm{T}_{h(a)} N$ coincides with the intersection

$$
\mathrm{T}_{a, h(a)} L \cap \mathrm{T}_{a} M
$$

and therefore can be written as a direct sum:

$$
\operatorname{Ker} h_{*, a}=E(a)+\mathfrak{h}_{b},
$$

where $b=h(a)$ and

$$
\mathfrak{h}_{b}=\left\{X \in \mathfrak{g} \mid \lambda(X)_{b}=0\right\}
$$

is the stability subalgebra of the point $b \in N$ with respect to the action $\lambda$.

Since $\lambda$ is a transitive action, we see that the image of $\mathrm{T}_{a} M$ by $h_{*, a}$ coincides with $\mathrm{T}_{b} N$. Thus, $h_{*, a}$ is an epimorphism and the inverse function theorem can be applied to $h$. Therefore, sets of the form

$$
M_{b}=h^{-1}(b)
$$

are smooth submanifolds of $M$.

From (13) it follows that for every $a \in M_{b}$ the subspace $E(a)$ belongs to the tangent space $\mathrm{T}_{a}\left(M_{b}\right)$. Thus, the distribution $E$ determines a completely integrable distribution on $M_{b}$. We shall still denote it by $E$. By construction vector fields $X \in \mathfrak{h}_{b}$ are tangent to the submanifold $M_{b}$ and therefore these fields are shuffling symmetries of $E$. In addition

$$
\operatorname{codim}_{M_{b}} E=\operatorname{dim} \mathfrak{h}_{b} .
$$

Definition. This transition from completely integrable distribution $E$ on the manifold $M$ to the distribution $E$ on $M_{b}$ determined by the mapping $h$ is called reduction of the symmetry algebra $\mathfrak{g}$ to the subalgebra $\mathfrak{h}=\mathfrak{h}_{b}$.

Let us make some observations about the procedure of reduction.

1) It is useful to assume that $N$ is a homogeneous space $N=G / H$, where $G$ is a connected Lie group, $\mathfrak{g}$ being its Lie algebra, and $H$ is the connected closed subgroup of $G$ corresponding to $\mathfrak{h}$. 
2) Finding of functions

$$
h: M \rightarrow G / H
$$

leading to maximal integral manifolds of the distribution $\widetilde{E}$ reduces to integration of ordinary differential equations on the homogeneous space $G / H$.

Indeed, let us fix points $a_{0} \in M$ and $b_{0} \in G / H$ and find mappings $h: M \rightarrow G / H$ such that $h\left(a_{0}\right)=b_{0}$ and $L_{h}$ is a maximal integral manifold of $\widetilde{E}$. For every $x \in M$ the value $h(x)$ can be found as a solution of a system of first-order differential equations with respect to $y(t) \in G / H$, where $y(t)=h(x(t))$ and $x(t)$ is an arbitrary path in $M$ (see Fig.22).

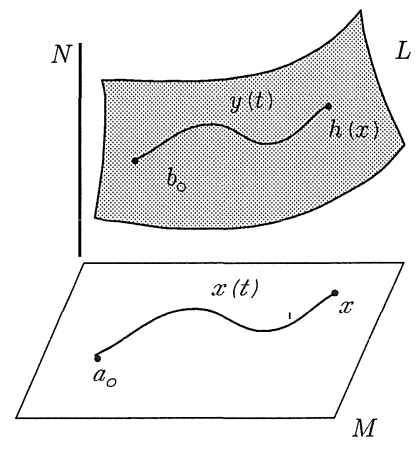

Fig. 22

By virtue of (11) the system has the form:

$$
\begin{aligned}
\left\langle\alpha_{1}, \dot{y}(t)\right\rangle & =\left\langle\alpha_{1}, \lambda\left(X_{1}\right)\right\rangle\left\langle\omega_{1}, \dot{x}(t)\right\rangle+\cdots+\left\langle\alpha_{1}, \lambda\left(X_{m}\right)\right\rangle\left\langle\omega_{m}, \dot{x}(t)\right\rangle, \\
\vdots & \\
\left\langle\alpha_{r}, \dot{y}(t)\right\rangle & =\left\langle\alpha_{r}, \lambda\left(X_{1}\right)\right\rangle\left\langle\omega_{1}, \dot{x}(t)\right\rangle+\cdots+\left\langle\alpha_{r}, \lambda\left(X_{m}\right)\right\rangle\left\langle\omega_{m}, \dot{x}(t)\right\rangle .
\end{aligned}
$$

\subsection{Examples.}

1) Let $\mathfrak{g}=\mathfrak{s l}(2, \mathbb{R})$ be the Lie algebra of $2 \times 2$ matrices with zero trace and $G=$ $S L(2, \mathbb{R})$ the Lie group of $2 \times 2$ matrices with determinant equal to 1 . Fix a vector $v_{0} \in \mathbb{R}^{2}$ and consider the subgroup

$$
H=\left\{A \in S L(2, \mathbb{R}) \mid A\left(v_{0}\right)=\lambda v_{0}\right\} .
$$

We shall assume that $v_{0}=(1,0)$. Then the homogeneous space $G / H$ can be identified with the projective line $\mathbb{R} P^{1}$. The corresponding isomorphism is determined by the mapping

$$
S L(2, \mathbb{R}) \ni A=\left[\begin{array}{ll}
a_{11} & a_{12} \\
a_{21} & a_{22}
\end{array}\right] \mapsto\left[a_{11}: a_{21}\right] \in \mathbb{R} P^{1} .
$$

Under this identification the natural action of $G$ on $G / H$ corresponds to linear fractional transformations of the projective line. The corresponding homomorphism 
$\lambda$ was calculated in example $2,1.3$. On the affine part $\mathbb{R}^{1}=\{[z: 1]\} \subset \mathbb{R} P^{1}$ it has the form:

$$
\begin{aligned}
h & =\left[\begin{array}{cc}
1 & 0 \\
0 & -1
\end{array}\right] \mapsto 2 z \frac{d}{d z}, \\
a^{+} & =\left[\begin{array}{ll}
0 & 1 \\
0 & 0
\end{array}\right] \mapsto \frac{d}{d z}, \\
a^{-} & =\left[\begin{array}{ll}
0 & 0 \\
1 & 0
\end{array}\right] \mapsto-z^{2} \frac{d}{d z} .
\end{aligned}
$$

In this case $r=\operatorname{dim} G / H=1$. Therefore, putting $r=1$ and $\alpha_{1}=d z$ in system (16), we obtain the Riccati equation

$$
z^{\prime}-2 a_{1} z-a_{2}+a_{3} z^{2}=0
$$

where $a_{i}=a_{i}(t)=\omega_{i}(\dot{x}(t))$.

The subalgebra $\mathfrak{h}$ corresponding to the Lie subgroup $H$ is solvable. Thus, the procedure of reduction allows to reduce integration of an arbitrary completely integrable distribution with $\mathfrak{s l}(2, \mathbb{R})$ being an algebra of shuffling symmetries to integration of the Riccati equation.

2) Let $V$ be an arbitrary $n$-dimensional vector space with a fixed flag of subspaces

$$
V=V_{n} \supset V_{n-1} \supset \cdots \supset V_{1} \supset V_{0}=\{0\}
$$

where $\operatorname{dim} V_{i}=i, i=0, \ldots, n$. Let $G$ be the Lie group of all linear isomorphisms of the flag:

$$
G=\left\{A \in G L(V) \mid A\left(V_{i}\right) \subset V_{i}, i=0, \ldots, n\right\}
$$

and $H \subset G$ the subgroup of isomorphisms constant on $V_{1}$ :

$$
H=\left\{A \in G \mid A(v)=v \forall v \in V_{1}\right\}
$$

Suppose $\left\{e_{1}, \ldots, e_{n}\right\}$ is a basis of $V$ such that the vectors $e_{1}, \ldots, e_{i}$ form a basis of $V_{i}$ for all $i=1, \ldots, n$. Then, in terms of matrices,

$$
\begin{gathered}
G=\left\{A \in G L(n, \mathbb{R}) \mid A=\left[\begin{array}{ccc}
\lambda_{1} & * & * \\
& \ddots & * \\
0 & & \lambda_{m}
\end{array}\right]\right\}, \\
H=\left\{A \in G \mid \lambda_{1}=1\right\} .
\end{gathered}
$$

In this case

$$
G / H=\mathbb{R}^{*}
$$

and this isomorphism is determined by the mapping

$$
G \ni A \mapsto \lambda_{1} \in \mathbb{R} \backslash\{0\},
$$


where $A\left(e_{1}\right)=\lambda_{1} e_{1}$.

The algebra $\mathfrak{g}$ of the Lie group $G$ is solvable and $\mathfrak{h} \subset \mathfrak{g}$ is a subalgebra of codimension 1. If $z$ denotes the standard coordinate on $\mathbb{R} \backslash\{0\} \subset \mathbb{R}$, then the action $\lambda: \mathfrak{g} \rightarrow \mathcal{D}(\mathbb{R})$ has the form

$$
\lambda(A)=\lambda_{1} z \frac{d}{d z}
$$

where $\lambda_{1}=\lambda_{1}(A)$ is the eigenvalue corresponding to the eigenvector $e_{1} \in V_{1}$.

In this case $r=\operatorname{dim} G / H=1$. Let us take the form

$$
\alpha_{1}=\frac{d z}{z}
$$

as a basis 1-form in (16). Then system (16) turns into an equation of the form:

$$
z^{\prime}=f(t)
$$

Similar considerations can be applied to any subalgebra of $\mathfrak{g}$ and therefore, by Lie's theorem, to an arbitrary solvable Lie algebra. Thus, the procedure of reduction, as well as the Lie-Bianchi theorem, allows to integrate distributions with solvable symmetry algebras by means of quadratures.

\section{§2. Model Equations AND The SUPerposition PRINCIPLE}

2.1. Differential equations 1.6. (16), appearing in the procedure of reduction of a symmetry algebra $\mathfrak{g}$ to a subalgebra $\mathfrak{h}$, are said to be model. There are two methods of constructing minimal models. First of them is specified by the minimal size of the system and therefore corresponds to homogeneous spaces $G / H$ such $\mathfrak{h}$ is a maximal subalgebra of $\mathfrak{g}$.

The second method is based on the fact that after solving model equations we need to solve a system of equations with the symmetry algebra $\mathfrak{h}$. Therefore, with the Lie-Bianchi theorem in mind, we should take the maximal solvable subalgebra for the subalgebra $\mathfrak{h}$. Models of the first type are called $d$-models, whereas those of the second type are called $r$-models.

Thus, the use of $d$-models allows to integrate the system by solving smaller systems of model equations several times, while the use of $r$-models allows to complete the integration by quadratures just after the first implementation of an $r$-model.

2.2. Model differential equations can be described formally by the following construction. Let $N=G / H$ be a homogeneous space and $\lambda: \mathfrak{g} \rightarrow \mathcal{D}(N)$ the natural representation of the Lie algebra $\mathfrak{g}$ by vector fields. Consider the path $\mathbb{R} \ni t \mapsto X_{t} \in \mathfrak{g}$ and the following vector field on the manifold $N \times \mathbb{R}$ :

$$
X=\frac{\partial}{\partial t}+\lambda\left(X_{t}\right)
$$

Systems of first-order differential equations on $N$ corresponding to fields (1) comprise, among other equations, all model equations. 
In the present section we shall consider only differential equations of this sort.

2.3. Let us give the coordinate expression for these systems. Let $\left\{X_{1}, \ldots, X_{m}\right\}$ be a basis in $\mathfrak{g}$ and $\left(y_{1}, \ldots, y_{n}\right)$ a local coordinate system on $G / H$. Assume that

$$
\left\{\begin{array}{l}
\lambda\left(X_{1}\right)=f_{1}^{1}(y) \frac{\partial}{\partial y_{1}}+\cdots+f_{1}^{n}(y) \frac{\partial}{\partial y_{n}} \\
\vdots \\
\lambda\left(X_{m}\right)=f_{m}^{1}(y) \frac{\partial}{\partial y_{1}}+\cdots+f_{m}^{n}(y) \frac{\partial}{\partial y_{n}}
\end{array}\right.
$$

and let $t \mapsto X_{t} \in \mathfrak{g}$ have the form

$$
X_{t}=A_{1}(t) X_{1}+\cdots+A_{m}(t) X_{m}
$$

Then the corresponding system of differential equations has the form (comp. 1.6. (16)):

$$
\left\{\begin{array}{l}
\dot{y}_{1}=A_{1}(t) f_{1}^{1}(y)+\cdots+A_{m}(t) f_{1}^{n}(y), \\
\vdots \\
\dot{y}_{n}=A_{1}(t) f_{1}^{n}(y)+\cdots+A_{m}(t) f_{m}^{n}(y) .
\end{array}\right.
$$

2.4. Example. Recall that (see $\S 1$ ) the action of the Lie algebra $\mathfrak{s l}(2, \mathbb{R})$ on the affine part of the projective line can be given by the vector fields

$$
\lambda\left(X_{1}\right)=\frac{\partial}{\partial y}, \lambda\left(X_{2}\right)=y \frac{\partial}{\partial y}, \lambda\left(X_{3}\right)=y^{2} \frac{\partial}{\partial y} .
$$

Let

$$
X_{t}=A_{1}(t) X_{1}+A_{2}(t) X_{2}+A_{3}(t) X_{3}
$$

be a path in $\mathfrak{s l}(2, \mathbb{R})$. In this case system (4) turns into the Riccati equation

$$
\dot{y}=A_{1}(t)+A_{2}(t) y+A_{3}(t) y^{2} .
$$

2.5. Let us consider another (less formal) method of obtaining systems of form (4).

Let $M$ be a certain $G$-space and $\lambda: \mathfrak{g} \rightarrow \mathcal{D}(M)$ the corresponding representation of $\mathfrak{g}$ by vector fields on the manifold $M$. Consider those systems of differential equations on $M$ whose solutions $y(t)$ all have the form

$$
y(t)=g(t)(y(0))
$$

for some path $\mathbb{R} \ni t \mapsto g(t) \in G, g(0)=e$, in the group $G$.

Let $\mathbb{R} \ni t \mapsto X_{t} \in \mathfrak{g}$ be the path in $\mathfrak{g}$ corresponding to the just-mentioned path in $G$. Identifying $\mathfrak{g}$ with the space $\mathrm{T}_{e} G$ tangent to $G$ at the identity $e \in G$, we obtain (see Fig.23)

$$
X_{t}=\left(g^{-1}(t)\right)_{*}(\dot{g})
$$




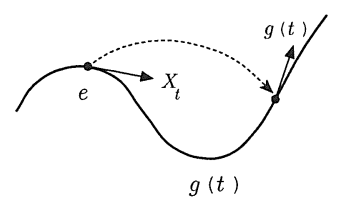

Fig. 23

Let us calculate tangent vectors $\dot{y}(t)$ to the trajectory $y(t)$ given by $(5)$. Differentiating relation (5) and using the chain rule, we obtain

$$
\dot{y}(t)=\lambda\left(X_{t}\right)_{y(t)}
$$

Thus, trajectories (5) are solutions of the system of form (4) corresponding to path (6). Note that since $y(0) \in M$ is arbitrary, relation (5) gives all solutions of the system.

Conversely, suppose that we are given a path $X_{t}$ in $\mathfrak{g}$. Then, considering relation (6) as a differential equation with respect to $g(t)$, we can find a unique path in $G$ such that $g(0)=e$. Thus, we arrive at the following description of systems (4):

Proposition. A system of first-order differential equations on $G / H$ has form (4) for some path $t \mapsto X_{t}$ in $\mathfrak{g}$ if and only if there exists a path $g(t), g(0)=e$, in $G$ such that all solutions of the system can be written in form (5).

2.6. So, integration of systems of form (4) and therefore of model systems reduces to finding a path $g(t)$ in the group $G$. Instead of solving equation (6) with respect to $g(t)$, we shall use another (more algebraic) method. Assume that $\mathfrak{g}$ is a simple Lie algebra and $\mathfrak{h}$ its maximal subalgebra.

Consider the action of the Lie group $G$ on the direct product $M^{k}=M \times \cdots \times M$ ( $k$ times), $M=G / H$,

$$
g:\left(a_{1}, \ldots, a_{k}\right) \mapsto\left(g\left(a_{1}\right), \ldots, g\left(a_{k}\right)\right)
$$

where $g \in G, a_{1}, \ldots, a_{k} \in M$.

Denote by $H(a) \subset G$ the stability subgroup of an element $a \in M$ and $H\left(a_{1}, \ldots\right.$, $\left.\ldots, a_{k}\right) \subset G$ the stability subgroup of an element $\left(a_{1}, \ldots, a_{k}\right) \in M^{k}$. It is obvious that

$$
H\left(a_{1}, \ldots, a_{k}\right)=H\left(a_{1}\right) \cap \cdots \cap H\left(a_{k}\right) .
$$

Let us show that a number $k$ can be chosen so that stability subgroups of almost all elements $\left(a_{1}, \ldots, a_{k}\right) \in M^{k}$ will be trivial. Consider stability subalgebras $\mathfrak{h}_{a_{i}}, i=$ $1, \ldots, k$. Let us choose an element $a_{2} \in M$ so that the dimension of the intersection $\mathfrak{h}_{a_{1}} \cap \mathfrak{h}_{a_{2}}$ is minimal. It is obvious that elements like this form an open and everywhere dense subset $M_{1}$ of $M$. Under the condition that $a_{2} \in M_{1}$, choose an element $a_{3} \in M$ so that the dimension of $\left(\mathfrak{h}_{a_{1}} \cap \mathfrak{h}_{a_{2}}\right) \cap \mathfrak{h}_{a_{3}}$ is minimal. The set $M_{2}$ of elements like this is also open and everywhere dense in $M$. We can similarly define everywhere dense 
sets $M_{i} \subset M$. Since $\mathfrak{h}$ is a maximal subalgebra of $\mathfrak{g}$,we see that the dimension of intersections

$$
\mathfrak{h}_{a_{1}} \cap \cdots \cap \mathfrak{h}_{a_{i}} \quad\left(a_{1} \in M, a_{2} \in M_{1}, \ldots, a_{i} \in M_{i-1}\right)
$$

monotonically decreases. Therefore, these intersections become zero, starting from some number $k$. Obviously

$$
k \leqslant \operatorname{dim} \mathfrak{h} .
$$

Then the subgroup $H\left(a_{1}, \ldots, a_{k}\right)$ is zero-dimensional.

Consider now the system of equations

$$
y_{1}^{0}=g\left(a_{1}\right), \ldots, y_{k}^{0}=g\left(a_{k}\right)
$$

with respect to elements $g \in G$ under the condition that $a_{1} \in M, a_{2} \in M_{1}, \ldots, a_{k} \in$ $M_{k-1}$. The set of solutions of the system is discrete.

In other words, fix an element $c=\left(c_{1}, \ldots, c_{k}\right) \in M^{k}$ of general position and consider the mapping

$$
\pi_{c}: G \rightarrow M^{k}
$$

defined by

$$
\pi_{c}(g)=\left(g\left(c_{1}\right), \ldots, g\left(c_{k}\right)\right) .
$$

Let $M_{c}=\pi_{c}(G)$ be the image of $\pi_{c}$. Then the projection

$$
\pi_{c}: G \rightarrow M_{c}
$$

is a fibration with fibre $H\left(c_{1}, \ldots, c_{k}\right)$.

Let us now revert to finding a path $g(t)$ satisfying condition (6). For this purpose consider a set of $k$ partial solutions $y_{1}^{0}(t), \ldots, y_{k}^{0}(t)$ of system $(7)$ such that the subgroup $H\left(c_{1}, \ldots, c_{k}\right)$, where $c_{1}=y_{1}^{0}(0), \ldots, c_{k}=y_{k}^{0}(0)$, is discrete. Put $c=\left(c_{1}, \ldots, c_{k}\right) \in M^{k}$.

The equations

$$
y_{1}^{0}(t)=g(t) c_{1}, \ldots, y_{k}^{0}(t)=g(t) c_{k},
$$

with respect to a path $g(t)$ in $G$ can be considered from a different standpoint. The function $Y: t \mapsto\left(y_{1}^{0}(t), \ldots, y_{k}^{0}(t)\right)$ gives a path in $M_{c}$, and we are looking for a path $g(t)$ in $G$ such that

$$
\pi_{c}(g(t))=Y(t) .
$$

According to properties of covering homotopies, a path $g(t)$ like this always exists and is uniquely determined by its value $g(0)$ at the point $t=0$. In our case $g(0)=e$ and, therefore, relations (10) uniquely determine a path $g(t)$ such that $g(0)=e$. Denote this path by

$$
g(t)=S\left(y_{1}^{0}(t), \ldots, y_{k}^{0}(t)\right) .
$$

Then the general solution $y(t)$ of system (7) has the form:

$$
y(t)=S\left(y_{1}^{0}(t), \ldots, y_{k}^{0}(t)\right) y(0) .
$$




\section{Definition.}

1) A system of partial solutions $y_{1}^{0}(t), \ldots, y_{k}^{0}(t)$ is called fundamental if the subgroup $H\left(y_{1}^{0}(0), \ldots, y_{k}^{0}(0)\right)$ of the group $G$ is discrete.

2) The function $S\left(y_{1}^{0}(t), \ldots, y_{k}^{0}(t)\right)$ is called the superposition function.

3) A homogeneous space $M=G / H$ is called $k$-stiff if $k$ is the minimal natural number such that for some $\left(a_{1}, \ldots, a_{k}\right) \in M^{k}$ the subgroup $H\left(a_{1}, \ldots, a_{k}\right)$ is discrete. The number $k$ is called the stiffness of $M$.

Summarizing results of this item, we obtain the following result:

Theorem. Let $k$ be the stiffness of the homogeneous space $M=G / H$. Then there exists a fundamental system of solutions $y_{1}^{0}(t), \ldots, y_{k}^{0}(t)$ of system (4) such that the general solution $y(t)$ of the system can be written in form (13), where $S=g(t)$ is the superposition function, which can be found from system (10).

2.7. Note that the group $G$ can be realized as a symmetry group of system (4) of differential equations. For this purpose consider various gauge transformations

$$
\begin{aligned}
& \hat{h}: M \times \mathbb{R} \rightarrow M \times \mathbb{R}, \\
& \hat{h}:(a, t) \mapsto(h(t) a, t)
\end{aligned}
$$

determined by paths $h(t)$ in $G$. A necessary and sufficient condition for a transformation of form (14) to be a symmetry of system (i)s that this transformation take every solution $y(t)$ of the system to a solution again. Using (13), we have

$$
\left.(t, y(t))=(t, g(t) y(0)) \stackrel{\hat{h}}{\mapsto}(t, h(t) g(t) y(0))=\left(t, g(t)\left(g^{-1}(t) h(t) g(t)\right)\right) y(0)\right)
$$

Therefore, a gauge transformation $h$ determines a symmetry if

$$
g^{-1}(t) h(t) g(t)=h_{0} \in G
$$

or

$$
h(t)=g(t) h_{0} y^{-1}(t) .
$$

Thus, relation (15) assigns to every element $h_{0}$ of the group $G$ a symmetry of system (4).

2.8. Remark. In the examples considered in $\S 1$ Lie groups act transitively on solution spaces. In this case systems of differential equations are called automorphic. Therefore, model equations are those automorphic systems of differential equations whose symmetry groups can be found with the help of a finite set of partial solutions (fundamental system of solutions).

2.9. In conclusion of this section let us describe model differential equations corresponding to one-dimensional homogeneous spaces. We shall first find out which finite-dimensional Lie algebras can be realized as transitive algebras on the line. 
Let $\mathfrak{g}$ be a Lie algebra of this sort and $\lambda: \mathfrak{g} \rightarrow \mathcal{D}(\mathbb{R})$ an injective homomorphism of Lie algebras. Then we can identify elements $X \in \mathfrak{g}$ with vector fields on the line. Every element $X \in \mathfrak{g}$ has the form:

$$
X=f(t) \frac{\partial}{\partial t}
$$

and it makes sense to speak about the order of an infinitesimal of $X$ at the zero point with the order of an infinitesimal of $f$ in mind.

The fact that $\mathfrak{g}$ acts transitively on the line means that there exists a vector field $X_{1} \in \mathfrak{g}$ such that $f(0) \neq 0$. Then, by the known theorem about straightening vector fields, a coordinate $z$ on the line can be (locally) chosen so that

$$
X_{1}=\frac{\partial}{\partial z}
$$

Denote by

$$
0 \leqslant k_{1}<k_{2}<\cdots<k_{r}
$$

the orders of an infinitesimal of vector fields belonging to $\mathfrak{g}$. From the commutation relation

$$
\left[f(z) \frac{\partial}{\partial z}, g(z) \frac{\partial}{\partial z}\right]=\left(f g^{\prime}-f^{\prime} g\right) \frac{\partial}{\partial z}
$$

it follows that if a function $f$ has zero of the maximal order $k_{r}$ and a function $g$ has zero of order $k_{i}(i<r)$, then their commutator has zero of order $k_{r}+k_{i}-1$. Therefore,

$$
k_{r}+k_{i}-1 \leqslant k_{r}
$$

for all $i=1, \ldots, r$. Relation (17) implies that $k_{i} \leqslant 1$ and therefore $r=\operatorname{dim} \mathfrak{g} \leqslant 3$.

Let us consider the cases $\operatorname{dim} \mathfrak{g}=1,2,3$ consecutively. The case $\operatorname{dim} \mathfrak{g}=1$ is trivial. Suppose $\operatorname{dim} \mathfrak{g}=2$. Then a basis of $\mathfrak{g}$ contains some vector field $X_{2}=f(z) \frac{\partial}{\partial z}$ in addition to $X_{1}$. Since the commutator

$$
\left[X_{1}, X_{2}\right]=f^{\prime}(z) \frac{\partial}{\partial z}
$$

is a linear combination of $X_{1}$ and $X_{2}$, we see that $X_{2}$ can be chosen as

$$
X_{2}=z \frac{\partial}{\partial z}
$$

If $\operatorname{dim} \mathfrak{g}=3$, then similar considerations show that the Lie algebra $\mathfrak{g}$ is generated by the vector fields $X_{1}$ and $X_{2}$ (see (16), (18)) and the vector field

$$
X_{3}=z^{2} \frac{\partial}{\partial z}
$$

Finally,we obtain the following result: 
Theorem.[S.Lie]. Suppose that a Lie algebra $\mathfrak{g}$ acts transitively on the line. Then, in a suitable (local) coordinate system, $\mathfrak{g}$ has one of the following forms:

a) $\mathfrak{g}$ is the commutative Lie algebra generated by the vector field

$$
X_{1}=\frac{\partial}{\partial z}
$$

b) $\mathfrak{g}$ is the solvable Lie algebra generated by the vector fields

$$
X_{1}=\frac{\partial}{\partial z}, X_{2}=z \frac{\partial}{\partial z}
$$

c) $\mathfrak{g}$ is the Lie algebra $\mathfrak{s l}(2, \mathbb{R})$ generated by the vector fields

$$
X_{1}=\frac{\partial}{\partial z}, X_{2}=z \frac{\partial}{\partial z}, X_{3}=z^{2} \frac{\partial}{\partial z}
$$

The corresponding differential equations have the form:

a) $\dot{z}=A_{1}(t)$

b) $\dot{z}=A_{1}(t)+A_{2}(t) z$

c) $\dot{z}=A_{1}(t)+A_{2}(t) z+A_{3}(t) z^{2}$.

2.10. Example. All differential equations listed in theorem 2.9 are Riccati equations. Let us illustrate finding of the superposition function by the example of the Riccati differential equation.

In this case, the action of the Lie group $S L(2, \mathbb{R})$ has the form:

$$
g(t) z=\frac{a_{11}(t) z+a_{12}(t)}{a_{21}(t) z+a_{22}(t)}
$$

if

$$
g(t)=A(t)=\left\|\begin{array}{ll}
a_{11}(t) & a_{12}(t) \\
a_{21}(t) & a_{22}(t)
\end{array}\right\| \in S L(2, \mathbb{R}) .
$$

It is known from projective geometry that every projective transformation of the line can be determined by the images of three different points. Therefore, the stiffness of $\mathbb{R} P^{1}$ equals $k=3$. To every number $z \in \mathbb{R}$ assign a vector of the plane:

$$
z \mapsto \bar{z}=\left(\begin{array}{c}
z \\
1
\end{array}\right)
$$

Then system (10) for finding $g(t)$ can be rewritten as

$$
\left\{\begin{array}{l}
A \bar{y}_{1}^{0}(t)=\lambda_{1} \bar{c}_{1}, \\
A \bar{y}_{2}^{0}(t)=\lambda_{2} \bar{c}_{2}, \\
A \bar{y}_{3}^{0}(t)=\lambda_{3} \bar{c}_{3}
\end{array}\right.
$$


where $\lambda_{1}, \lambda_{2}, \lambda_{3}$ are nonzero numbers.

Denote by $Y_{i j}$ (respectively, $C_{i j}$ ) the $2 \times 2$ matrix with the vectors $\bar{y}_{i}^{0}, \bar{y}_{j}^{0}$ (respectively, $\bar{c}_{i}, \bar{c}_{j}$ ) being its columns. Then system (20) is equivalent to the matrix system

$$
A Y_{i j}=\Lambda_{i j} C_{i j}
$$

where

$$
\Lambda_{i j}=\left[\begin{array}{cc}
\lambda_{i} & 0 \\
0 & \lambda_{j}
\end{array}\right], i, j=1,2,3
$$

From (21) we obtain

$$
\lambda_{i} \lambda_{j}=\operatorname{det} Y_{i j} / \operatorname{det} C_{i j}
$$

so that

$$
\begin{aligned}
& \lambda_{1}^{2}=\operatorname{det} Y_{12} \operatorname{det} Y_{13} \operatorname{det} C_{23} /\left(\operatorname{det} C_{12} \operatorname{det} C_{13} \operatorname{det} Y_{23}\right), \\
& \lambda_{2}^{2}=\operatorname{det} Y_{12} \operatorname{det} C_{13} \operatorname{det} Y_{23} /\left(\operatorname{det} C_{12} \operatorname{det} Y_{13} \operatorname{det} C_{23}\right), \\
& \lambda_{3}^{2}=\operatorname{det} C_{12} \operatorname{det} Y_{13} \operatorname{det} Y_{23} /\left(\operatorname{det} Y_{12} \operatorname{det} C_{13} \operatorname{det} C_{23}\right),
\end{aligned}
$$

and

$$
A=\Lambda_{12} C_{12} Y_{12}^{-1}
$$

§3. Model equations: The Series $A_{k}, B_{k}, D_{k}$

3.1. Let $V$ be a vector space (over $\mathbb{R}$ or $\mathbb{C}$ ) of dimension $n+k, n \geqslant 2$. The grassmannian $G_{n+k, k}$ of $k$-dimensional subspaces is the set whose elements are $k$ dimensional subspaces $L \subset V$. The Lie group $\mathrm{SL}(V)=\mathrm{SL}(n+k)$ acts naturally on this set:

$$
A: G_{n+k, k} \ni L \mapsto A(L) \in G_{n+k, k}
$$

for all subspaces $L$ and transformations $A \in \mathrm{SL}(n+k)$. This action is transitive. The stability subgroup $H(L)$ of an element $L \in G_{n+k, k}$ has the form:

$$
H(L)=\{A \in \mathrm{SL}(V) \mid A(L)=L\} .
$$

Thus,

$$
G_{n+k, k} \approx \mathrm{SL}(n+k) / H(L)
$$

3.2. Let us give coordinate description of constructions of the previous item. To be definite, assume that $V$ is a vector space over $\mathbb{R}$. Let us identify $V$ with $\mathbb{R}^{n+k}$ and let $L_{0}$ be a fixed $k$-dimensional subspace of $V$. We identify $L_{0}$ with the space $\mathbb{R}^{k}$ embedded into $\mathbb{R}^{n+k}$ :

$$
\mathbb{R}^{k} \ni\left(x_{1}, \ldots, x_{k}\right) \mapsto\left(x_{1}, \ldots, x_{k}, 0, \ldots, 0\right) \in \mathbb{R}^{n+k} .
$$

Under this identification, all elements $L \in G_{n+k, k}$ lying in a sufficiently small neighborhood of the point $L_{0} \in G_{n+k, k}$ can be represented as $k \times n$ matrices

$$
W=\left(w_{i j}\right)_{\substack{1 \leqslant i \leqslant k \\ 1 \leqslant j \leqslant n}},
$$


where the vectors

$$
\begin{aligned}
& \left(1,0, \ldots, 0, w_{11}, w_{12}, \ldots, w_{1 n},\right) \\
& \left(0,1, \ldots, 0, w_{21}, w_{22}, \ldots, w_{2 n},\right) \\
& \vdots \\
& \left(0,0, \ldots, 1, w_{k 1}, w_{k 2}, \ldots, w_{k n}\right)
\end{aligned}
$$

form a basis of a subspace $L$.

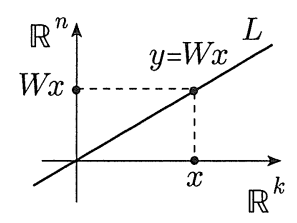

Fig. 24

Each vector $z \in \mathbb{R}^{n+k}$ can be written as a column:

$$
z=\left(\begin{array}{l}
y \\
x
\end{array}\right)
$$

where $y \in \mathbb{R}^{n}$ and $x \in \mathbb{R}^{k}$. Then a space $L \in G_{n+k, k}$ is the graph of the corresponding linear mapping $W: \mathbb{R}^{k} \rightarrow \mathbb{R}^{n}, x \mapsto y=W x$.

Each element $A$ of the group $\mathrm{SL}(n+k)$ in these coordinates can be written as a hypermatrix:

$$
A=\left[\begin{array}{ll}
A_{11} & A_{12} \\
A_{21} & A_{22}
\end{array}\right]
$$

where $A_{11}$ is a matrix of order $n \times n, A_{22}$ a $k \times k$ matrix, $A_{12}$ an $n \times k$ matrix, $A_{21}$ a $k \times n$ matrix, and $\operatorname{det} A=1$.

Elements of the stability subgroup $H\left(L_{0}\right)$ are blocked triangular matrices of the form:

$$
A=\left[\begin{array}{cc}
A_{11} & 0 \\
A_{21} & A_{22}
\end{array}\right] .
$$

The action of an element $A \in \mathrm{SL}(n+k)$ in coordinates $W$ has the form

$$
\begin{array}{r}
L\left(\begin{array}{c}
y=W x \\
x
\end{array}\right) \mapsto\left(\begin{array}{ll}
A_{11} & A_{12} \\
A_{21} & A_{22}
\end{array}\right)\left(\begin{array}{c}
y \\
x
\end{array}\right)=\left(\begin{array}{c}
A_{11} y+A_{12} x \\
A_{21} y+A_{22} x
\end{array}\right)= \\
=\left(\begin{array}{c}
\left(A_{11} W+A_{12}\right) x \\
\left(A_{21} W+A_{22}\right) x
\end{array}\right)=\left(\begin{array}{c}
W_{A} x^{\prime} \\
x^{\prime}
\end{array}\right),
\end{array}
$$

where

$$
W_{A}=\left(A_{11} W+A_{12}\right)\left(A_{21} W+A_{22}\right)^{-1},
$$


so that

$$
A: W \rightarrow W_{A}=\left(A_{11} W+A_{12}\right)\left(A_{21} W+A_{22}\right)^{-1} .
$$

3.3. Let us describe the corresponding action

$$
\lambda: \mathfrak{s l}(n+k) \rightarrow \mathcal{D}\left(G_{n+k, k}\right)
$$

of the Lie algebra $\mathfrak{s l}(n+k)$ on the grassmannian $G_{n+k, k}$.

Each element $h \in \mathfrak{s l}(n+k)$ is a hypermatrix of the form

$$
h=\left[\begin{array}{ll}
h_{11} & h_{12} \\
h_{21} & h_{22}
\end{array}\right]
$$

with zero trace: $\operatorname{tr} h_{11}+\operatorname{tr} h_{22}=0$.

Let us calculate the value of a vector field $\lambda(h)$ at a point $W$. We have

$$
\lambda(h)_{W}=\left.\frac{d}{d t}\right|_{t=0}\left[\left(h_{11}(t) W+h_{12}(t)\right)\left(h_{21}(t) W+h_{22}(t)\right)^{-1}\right],
$$

where

$$
\left[\begin{array}{ll}
h_{11}(t) & h_{12}(t) \\
h_{21}(t) & h_{22}(t)
\end{array}\right]=\exp t h
$$

Therefore,

$$
\left.\frac{d h_{i j}(t)}{d t}\right|_{t=0}=h_{i j}, \quad h_{i j}(0)=\delta_{i j}
$$

and

$$
\lambda(h)_{W}=h_{11} W+h_{12}-W\left(h_{21} W+h_{22}\right) .
$$

Thus, the field $\lambda(h)$ in coordinates $W$ has the form

$$
\begin{gathered}
\lambda(h)_{W}=\left(h_{12}+h_{11} W-W h_{22}-W h_{21} W\right) \frac{\partial}{\partial W} \\
\operatorname{tr} h_{11}+\operatorname{tr} h_{22}=0 .
\end{gathered}
$$

3.4. The differential equation corresponding to the action $\lambda$ has the form:

$$
\dot{W}=B_{12}(t)+B_{11}(t) W+W B_{22}(t)+W B_{21}(t) W
$$

where $\operatorname{tr} B_{11}=\operatorname{tr} B_{22}$. It is the matrix Riccati equation.

The general solution $W(t)$ of equation (5) can be written as

$$
W(t)=\left(A_{11}(t) W(0)+A_{12}(t)\right)\left(A_{21}(t) W(0)+A_{22}(t)\right)^{-1},
$$

where matrices $A(t)=\left\|A_{i j}(t)\right\| \in \mathrm{SL}(n+k)$ are the same for all solutions. 
Suppose $r$ is the stiffness of the grassmannian and $W_{1}(t), \ldots, W_{r}(t)$ are partial solutions of (5). Then the matrices $A(t)$ can be found from the following system of linear equations

$$
A_{11} W_{l}(0)+A_{12}=W_{l}(t)\left(A_{21} W_{l}(0)+A_{22}\right), l=1, \ldots, r .
$$

3.5. Example. If $k=1$, then the grassmannian $G_{n+1,1}$ coincides with the $n$ dimensional projective space. Each projective transformation of $\mathbb{R P}^{n}$ is completely determined by images of $(n+2)$ points of general position. Therefore, the stiffness of $\mathbb{R P}^{n}$ equals $n+2$. In this case it can be assumed that $r=n+2$ in system (7). The procedure of explicit solution of the system is similar to that for the case $n=1$ considered before.

3.6. Let $k=n$. Then all matrices in Riccati equation (5) are square matrices of order $n \times n$. Let us find the stiffness of the grassmannian $G_{2 n, n}$. Let $L_{0}, L_{1} \in G_{2 n, n}$ be the subspaces corresponding to the horizontal and vertical planes (see Fig. 24) respectively. The stability subgroup $H\left(L_{0}, L_{1}\right)=H\left(L_{0}\right) \cap H\left(L_{1}\right)$ consists of blocked diagonal matrices of the form:

$$
A=\left[\begin{array}{cc}
A_{11} & 0 \\
0 & A_{22}
\end{array}\right],
$$

where $\operatorname{det} A_{11} \cdot \operatorname{det} A_{22}=1$. The action of elements $A \in H\left(L_{0}, L_{1}\right)$ in coordinates $W$ has the form:

$$
A: W \mapsto W_{A}=A_{11} W A_{22}^{-1} .
$$

For a third point $L_{2}$, take the subspace corresponding to the diagonal $y=x$. Then

$$
H\left(L_{0}, L_{1}, L_{2}\right)=\left\{\left[\begin{array}{cc}
B & 0 \\
0 & B
\end{array}\right] \mid B \in \mathrm{SL}(n)\right\}
$$

and the action of this stability subgroup has the form:

$$
W \mapsto B W B^{-1} .
$$

It remains to note that, with respect to this action, the common stabilizer of two matrices $W_{1}$ and $W_{2}$ of general position is trivial. Thus, it is sufficient to use 5 solutions "of general position" in order to find the general solution with the help of relations (6) and (7).

3.7. Expression (1) has a universal nature and holds for other classical Lie groups. In conclusion of this section, let us consider the series $D_{k}$ and $B_{k}$. Assume that a vector space $V$ has dimension $n+k$ and is supplied with an Euclidean metric $g$. Let $G=\mathrm{SO}(g)$ be the group of transformations of $V$ preserving the metric $g$. Let us fix an orthonormal basis in $V$ and identify $V$ with $\mathbb{R}^{n+k}$ and $g$ with the standard scalar product:

$$
g(X, Y)=(X, Y)=x_{1} y_{1}+\cdots+x_{n+k} y_{n+k}
$$


Then the Lie group $\mathrm{SO}(g)=\mathrm{SO}(n+k)$ can be identified with the group of orthogonal matrices of determinant 1 , while the Lie algebra $\mathfrak{s o}(g)$ with the algebra of skewsymmetric matrices.

The action of $\mathrm{SO}(n+k)$ on the grassmannian $G_{n+k, k}$ is transitive. In coordinates $W$ this action also has form (1). For a space complementary to $L_{0}$ consider the orthogonal complement $L_{0}^{\perp}$. Then a hypermatrix

$$
h=\left[\begin{array}{ll}
h_{11} & h_{12} \\
h_{21} & h_{22}
\end{array}\right]
$$

belongs to $\mathfrak{s o}(n+k)$ if and only if the following conditions hold:

$$
h_{11}^{t}+h_{11}=0, \quad h_{22}^{t}+h_{22}=0, \quad h_{12}^{t}+h_{21}=0 .
$$

The vector field $\lambda(h)$ in coordinates $W$ has the form:

$$
\lambda(h)=\left(h_{12}+h_{11} W-W h_{22}+W h_{12}^{t} W\right) \frac{\partial}{\partial W} .
$$

The corresponding differential equation has the form:

$$
\dot{W}=B_{12}(t)+B_{11}(t) W+W B_{22}(t)+W B_{21}^{t}(t) W .
$$

Here $W$ is a matrix of order $k \times k$ and $B_{11}, B_{22}$ are skew-symmetric matrices. Equation (11) is called the orthogonal Riccati equation.

3.8. A fundamental system of solutions and general solution of the orthogonal Riccati equation can be found in much the same way as in the general matrix case. We shall restrict ourselves to evaluating the number of fundamental solutions of the orthogonal Riccati equation in the case of $n \times n$ matrices; in other words, we shall find the stiffness of $G_{2 n, n}$ with respect to the orthogonal group.

It is obvious that elements of the stability subgroup $H\left(L_{0}\right)$ have the form:

$$
A=\left[\begin{array}{cc}
A_{11} & 0 \\
0 & A_{22}
\end{array}\right]
$$

where $A_{11}$ and $A_{22}$ are orthogonal matrices of order $n \times n$.

Let $L_{1}$ be the subspace corresponding to the diagonal $y=x$ (cp 3.6). Then the stability subgroup $H\left(L_{0}, L_{1}\right)$ consists of matrices of the form:

$$
A=\left[\begin{array}{ll}
B & 0 \\
0 & B
\end{array}\right],
$$

where $B$ is an orthogonal matrix of order $n \times n$. The action of elements of $H\left(L_{0}, L_{1}\right)$ in coordinates $W$ has the form:

$$
W \mapsto B W B^{t} .
$$

Therefore, for any two subspaces $L_{2}$ and $L_{3}$ of general position, the stability subgroup $H\left(L_{0}, L_{1}, L_{2}, L_{3}\right)$ is trivial.

Thus the stiffness of $G_{2 n, n}$ equals 4 . 
§4. Model Equations: The SERIES $C_{n}$

4.1. Let $V$ be a symplectic space of dimension $2 n$ with a structure 2 -form $\omega$. Fixing a symplectic basis in $V$, we identify $V$ with the arithmetic space $\mathbb{R}^{2 n}$ where $\omega$ has the form:

$$
\omega(X, Y)=\left(x_{1} y_{n+1}-x_{n+1} y_{1}\right)+\cdots+\left(x_{n} y_{2 n}-x_{2 n} y_{n}\right)
$$

Let $G=\operatorname{Sp}(2 n)$ be the group of symplectic transformations of $V$; in other words, $G$ is a group of transformations $A: V \rightarrow V$ such that

$$
\omega(A X, A Y)=\omega(X, Y)
$$

for all vectors $X, Y \in \mathbb{R}^{2 n}$.

If $($,$) is the standard scalar product in \mathbb{R}^{2 n}$, then the form $\omega$ can be replaced by the operator $\Omega$ such that

$$
\omega(X, Y)=(\Omega X, Y)
$$

The operator $\Omega$ is skew-symmetric and its matrix has the form:

$$
\Omega=\left[\begin{array}{cc}
0 & I_{n} \\
-I_{n} & 0
\end{array}\right]
$$

where $I_{n}$ is the identity matrix of order $n \times n$.

Condition (2) for a transformation $A$ to be symmetric can be written in matrix form as

$$
A \Omega A^{t}=\Omega
$$

Then the Lie algebra $\mathfrak{s p}(2 n)$ can be written in matrix form as

$$
\mathfrak{s p}(2 n)=\left\{h \in \mathfrak{g l}(2 n) \mid h \Omega+\Omega h^{t}=0\right\} .
$$

Let $h$ be a hypermatrix

$$
h=\left[\begin{array}{ll}
h_{11} & h_{12} \\
h_{21} & h_{22}
\end{array}\right] .
$$

Then the condition $h \Omega+\Omega h^{t}=0$ is equivalent to the following conditions:

$$
h_{11}+h_{22}^{t}=0, \quad h_{12}=h_{12}^{t}, \quad h_{21}=h_{21}^{t} .
$$

Thus, elements of $\mathfrak{s p}(2 n)$ can be written as hypermatrices of the form:

$$
h=\left[\begin{array}{cc}
h_{11} & h_{12} \\
h_{21} & -h_{11}^{t}
\end{array}\right]
$$

where $h_{12}, h_{21}$ are symmetric matrices of order $n \times n$ and $h_{11}$ is an arbitrary matrix of order $n \times n$. Correspondingly, if we write a matrix $A \in \operatorname{Sp}(2 n)$ as a hypermatrix:

$$
A=\left[\begin{array}{ll}
A_{11} & A_{12} \\
A_{21} & A_{22}
\end{array}\right],
$$


then condition (4) will take the form:

$$
A_{11} A_{22}^{t}-A_{12} A_{12}^{t}=I_{n}, \quad A_{12} A_{11}^{t}-A_{11} A_{12}^{t}=0, \quad A_{21} A_{22}^{t}-A_{22} A_{21}^{t}=0 .
$$

4.2. If $1<k<2 n-1$, then the action of $\operatorname{Sp}(2 n)$ on the grassmannian $G_{2 n, k}$ is not transitive. In this case two subspaces $L_{1}, L_{2} \in G_{2 n, k}$ lie in the same orbit if and only if the rank of the restrictions of the structure form $\omega$ to $L_{1}$ and $L_{2}$ is the same. We shall consider the most interesting case - the case of the Lagrange grassmannian.

Definition. A subspace $L \subset V$ of dimension $(\operatorname{dim} V) / 2$ is called a Lagrange subspace if the restriction of the structure form $\omega$ to $L$ is trivial, i.e.

$$
\omega(X, Y)=0
$$

for all vectors $X, Y \in L$.

Denote by $\Lambda_{n}$ the set of all Lagrange subspaces in $\left(\mathbb{R}^{2 n}, \omega\right)$. The set $\Lambda_{n}$ is called the Lagrange grassmannian.

Witt's theorem shows that the action of $\operatorname{Sp}(2 n)$ on $\Lambda_{n}$ is transitive.

4.3. Let us describe the action $\lambda: \mathfrak{s p}(2 n) \rightarrow \mathcal{D}\left(\Lambda_{n}\right)$ in coordinates $W$, assuming that the subspace $L_{0}$ is spanned by the first $n$ basis vectors, while its complement is spanned by the last $n$ vectors.

Lemma. The subspace $y=W x$ determined by a matrix $W$ is a Lagrange subspace if and only if $W$ is a symmetric matrix.

Proof. Suppose $\left\{e_{1}, \ldots, e_{n}, e_{n+1}, \ldots, e_{2 n}\right\}$ is a standard basis in $\mathbb{R}^{2 n}$. Let us calculate the value of the structure form $\omega$ at a pair of vectors $X=e_{i}+W\left(e_{i}\right)$ and $Y=$ $e_{j}+W\left(e_{j}\right)$, where $i, j \leqslant n$. We have

$$
\omega(X, Y)=\omega\left(e_{i}, W\left(e_{j}\right)\right)+\omega\left(W\left(e_{i}\right), e_{j}\right)=W_{i j}-W_{j i}=0 .
$$

Therefore,

$$
W^{t}=W
$$

4.4. Using expression (6) for elements of the Lie algebra $\mathfrak{s p}(2 n)$, we see that vector fields $\lambda(h)$ on the space of symmetric $n \times n$ matrices $W$ have the form:

$$
\lambda(h)=\left(h_{12}+h_{11} W+W h_{11}^{t}-W h_{21} W\right) \frac{\partial}{\partial W} .
$$

The corresponding system of differential equations has the form:

$$
\dot{W}=B_{12}(t)+B_{11}(t) W+W B_{11}^{t}(t)-W h_{21}(t) W
$$

where $W=W(t), B_{12}(t), B_{21}(t)$ are symmetric $n \times n$ matrices and $B_{11}$ is an arbitrary $n \times n$ matrix. Equation (9) is called the symplectic Riccati equation. 
4.5. A fundamental system of solutions of equation (9) can be found in the same way as in the case of the general Riccati equation. Let us evaluate the stiffness of the Lagrange grassmannian $\Lambda_{n}$ with respect to the symplectic group, assuming that $n \geqslant 2$.

Let us choose Lagrange subspaces $L_{0}$ and $L_{1}$ as in the general case. Then the stability subgroup $H\left(L_{0}, L_{1}\right)$ consists of symplectic matrices of the form:

$$
A=\left[\begin{array}{cc}
A_{11} & 0 \\
0 & A_{22}
\end{array}\right] \text {. }
$$

From conditions (7) it follows that

$$
H\left(L_{0}, L_{1}\right)=\left\{A=\left[\begin{array}{cc}
B & 0 \\
0 & \left(B^{t}\right)^{-1}
\end{array}\right] \mid B \in \mathrm{GL}(n)\right\} .
$$

The action of elements $A \in H\left(L_{0}, L_{1}\right)$ in coordinates $W$ has the form:

$$
A: W \mapsto B W B^{t} .
$$

Therefore, for any two Lagrange subspaces $L_{2}$ and $L_{3}$ of general position, the stability subgroup $H\left(L_{0}, L_{1}, L_{2}, L_{3}\right)$ is trivial. Thus, the symplectic Riccati equation, as well as the orthogonal one, has a system of 4 fundamental solutions regardless of the dimension of the space $(2 n \geqslant 4)$.

4.6. Remark. If $n=1$, the group $\operatorname{Sp}(2)$ coincides with the group $\mathrm{SL}(2)$ and the space $\Lambda_{1}$ with the projective line. Therefore, in this case the number of fundamental solutions of the Riccati equation equals three. 